Florida International University FIU Digital Commons

$3-21-2012$

\title{
Measurement of the Induced Polarization of Lambda(1116) in Kaon Electroproduction with CLAS.
}

Marianna Gabrielyan

Florida International University, mgabr001@fiu.edu

DOI: $10.25148 /$ etd.FI12050225

Follow this and additional works at: https://digitalcommons.fiu.edu/etd

\section{Recommended Citation}

Gabrielyan, Marianna, "Measurement of the Induced Polarization of Lambda(1116) in Kaon Electroproduction with CLAS." (2012). FIU Electronic Theses and Dissertations. 619.

https://digitalcommons.fiu.edu/etd/619 


\title{
FLORIDA INTERNATIONAL UNIVERSITY
}

Miami, Florida

\section{MEASUREMENT OF THE INDUCED POLARIZATION OF $\Lambda(1116)$ IN KAON ELECTROPRODUCTION WITH CLAS}

\author{
A dissertation submitted in partial fulfillment of the \\ requirements for the degree of \\ DOCTOR OF PHILOSOPHY \\ in \\ PHYSICS \\ by
}

Marianna Y. Gabrielyan

2012 
To: Dean Kenneth Furton

College of Arts and Sciences

This dissertation, written by Marianna Y. Gabrielyan, and entitled Measurement of the Induced Polarization of $\Lambda(1116)$ in Kaon Electroproduction with CLAS, having been approved in respect to style and intellectual content, is referred to you for judgement.

We have read this thesis and recommend that it be approved.

Joerg Reinhold

Misak Sargsian

Julian Edward

Brian Raue, Major Professor

Date of Defense: March 21, 2012

The dissertation of Marianna Y. Gabrielyan is approved.

Dean Kenneth Furton

College of Arts and Sciences

Dean Lakshmi N. Reddi

University Graduate School

Florida International University, 2012 


\section{DEDICATION}

To my loving parents

Marietta Matevosyan and Yura Gabrielyan. 


\section{ACKNOWLEDGMENTS}

First and foremost, I would like to thank my adviser Dr. Brian Raue for his patient support, expert guidance and trust towards me, without which this work would have not been possible. I would like to thank him for always being available to me to answer all my questions and for being not only a great mentor but also an excellent teacher and friend. I truly appreciate his patience and tolerance towards me during my study at FIU. And for all this I'm eternally indebted.

I thank the members of my committee for their support and advice at various stages of writing of this dissertation.

I would like to thank Dr. Daniel S. Carman for his help in understanding the CLAS detector, his excellent guidence throughout various stages of the analysis and for his constructive criticism and help in preparing my presentations and talks. I would also like to thank Dr. Eugene Pasyuk for his expert wisdom and help in developing my skills in working with PAW.

My graduate studies would not have been the same without academic discussions,

emotional support and encouragement from all my friends and student-colleagues. I highly value their friendship which has made my years at FIU extraordinarily pleasant and memorable.

I am very grateful to all of the faculty and staff of the FIU Physics Department. I am fortunate to have had the opportunity to work with a group of enthusiastic and knowledgeable people, who share a common passion for science. I have enjoyed every moment that we have worked together.

I would like to thank the Florida International University Graduate School for their assistance and for the support provided by their Dissertation Year Fellowship award program.

I would like to give special thanks to my friends Blanche and Angelo Grassi for making me feel at home away from home.

Finally, I would like to thank my parents and all my family members whose unconditional love and support helped me to get through difficult times without giving up. 
Thanks to them I have come to understand and appreciate the importance of a good education, hard work and patient persistence towards pursuing my goals.

This has been an extraordinary journey for which I would like to thank you all. 


\section{ABSTRACT OF THE DISSERTATION ELECTROPRODUCTION WITH CLAS \\ by \\ Marianna Y. Gabrielyan \\ Florida International University, 2012 \\ Miami, Florida \\ Professor Brian Raue, Major Professor}

MEASUREMENT OF THE INDUCED POLARIZATION OF $\Lambda(1116)$ IN KAON

The CLAS Collaboration is using the $p\left(e, e^{\prime} K^{+} p\right) \pi^{-}$reaction to perform a measurement of the induced polarization of the electroproduced $\Lambda(1116)$. The parity-violating weak decay of the $\Lambda$ into $p \pi^{-}$(64\%) allows extraction of the recoil polarization of the $\Lambda$. The present study uses the CEBAF Large Acceptance Spectrometer (CLAS) to detect the scattered electron, the kaon, and the decay proton. CLAS allows for a large kinematic acceptance in $Q^{2}\left(0.8 \leq Q^{2} \leq 3.5 \mathrm{GeV}^{2}\right), W(1.6 \leq W \leq 3.0 \mathrm{GeV})$, as well as the kaon scattering angle. In this experiment a $5.499 \mathrm{GeV}$ electron beam was incident upon an unpolarized liquid-hydrogen target. The goal is to map out the kinematic dependencies for this polarization observable to provide new constraints for theoretical models of the electromagnetic production of kaon-hyperon final states. Along with previously published photo- and electroproduction cross sections and polarization observables from CLAS, SAPHIR, and GRAAL, these data are needed in a coupled-channel analysis to identify previously unobserved $s$-channel resonances. 


\section{TABLE OF CONTENTS}

CHAPTER

PAGE

1. Introduction 1

1.1 Historical Overview . . . . . . . . . . . . . . . . 1

1.2 Motivation ....................... 4

1.3 Physics Variables and Formalism . . . . . . . . . . . . . . . . 8

$1.4 \Lambda$ Polarization . . . . . . . . . . . . . . . . . . . . . . . . 16

1.5 Theoretical Models . . . . . . . . . . . . . . . . . . . . . . . . 20

1.6 Previous Experimental Data . . . . . . . . . . . . . . . . . . 24

2. Experimental Setup $\mathbf{2 6}$

2.1 Facility Overview . . . . . . . . . . . . . . . . . . . . . 26

2.2 CLAS Detector . . . . . . . . . . . . . . . . . . . 27

2.3 Cryogenic Target . . . . . . . . . . . . . . . . . . . 36

2.4 Data Acquisition and Trigger System . . . . . . . . . . . . . . . 37

3. Particle Identification 39

3.1 Electrons . . . . . . . . . . . . . . . . . . . . . . . 39

3.2 Hadrons . . . . . . . . . . . . . . . . . . . . . . . 50

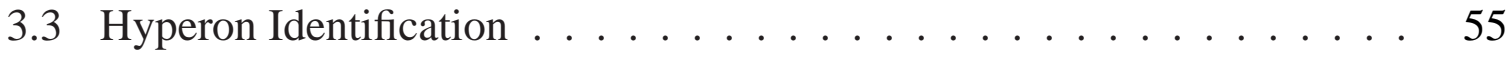

4. Analysis 59

4.1 Cooking and Data Reduction . . . . . . . . . . . . . . . . 59

4.2 Binning . . . . . . . . . . . . . . . . . . 60

4.3 Momentum Corrections . . . . . . . . . . . . . . . . . . . . 61

4.4 Background Subtraction . . . . . . . . . . . . . . . . . . . 71

4.5 Acceptance Corrections . . . . . . . . . . . . . . . . . . . . . . . 79

4.6 Recoil Polarization Calculation . . . . . . . . . . . . . . . . . . . 84

4.7 Radiative Corrections . . . . . . . . . . . . . . . . . . . . . . 86

$4.8 Q^{2}$ Dependence Study . . . . . . . . . . . . . . . . . . . . . 87

5. Systematic Uncertainties 89

5.1 Acceptance Corrections . . . . . . . . . . . . . . . . . . . . . . . . . . . 89

5.2 Pion Missing Mass Cut . . . . . . . . . . . . . . . . . . . . . . . 91

5.3 Geometrical Fiducial Cuts . . . . . . . . . . . . . . . . . . . . 91

5.4 Proton Acceptance Corrections with Polarized MC . . . . . . . . . . . . 92

$5.5 t$-Slope . . . . . . . . . . . . . . . . . . . . . 96

5.6 Fit Method . . . . . . . . . . . . . . . . . . . . . . . . . . 97

5.7 Fixed vs. Float Fit Parameters . . . . . . . . . . . . . . . . . . . . . . 98

5.8 PID Routine . . . . . . . . . . . . . . . . . . . . . . . . . . . . 98

5.9 Deviations of $P_{L}$ and $P_{T}$ from Zero . . . . . . . . . . . . . . 99

5.10 Sector Dependence . . . . . . . . . . . . . . . . . . . . 100

6. Results and Conclusions 105

$6.1 \quad \Lambda$ Recoil Polarization . . . . . . . . . . . . . . . . . . . . . 105

6.2 Comparison to Theoretical Models . . . . . . . . . . . . . . . . . . . . 109

6.3 Comparison to Previous Experimental Results . . . . . . . . . . . . . 110 


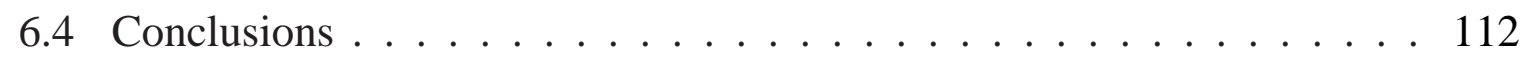

$\begin{array}{ll}\text { APPENDICES } & 114\end{array}$

LIST OF REFERENCES 126

$\begin{array}{lr}\text { VITA } & 128\end{array}$ 


\section{LIST OF FIGURES}

FIGURE

PAGE

2.1 Schematic view of the Jefferson Lab accelerator [36]. . . . . . . . . . .

2.2 Schematic view of the CLAS detector showing all subsystems of the

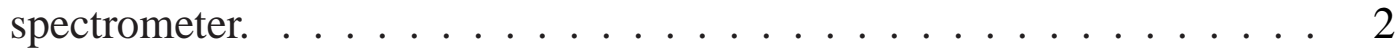

2.3 Schematic diagram of the CLAS detector. The relative positioning of the main and mini-torus coils are shown with respect to the target position. .

2.4 Typical CLAS event reconstruction. Negatively charged particles are bent towards the beamline. Track segments are combined in different superlayers of all regions to form the charged particle trajectories that traverse the volume of the CLAS spectrometer. . . . . . . . . . . 3

2.5 CC Optical mirror system. . . . . . . . . . . . . . . . . . . . 32

2.6 CLAS electromagnetic calorimeter modules. U, V and W layers are shown. 34

2.7 CLAS event reconstruction by EC . . . . . . . . . . . . . . 35

2.8 TOF scintillator paddles for one sector. . . . . . . . . . . . . . . 36

2.9 E1F cryogenic target. . . . . . . . . . . . . . . . . . . 37

3.1 Reconstructed $\Lambda$ mass with reduced (red curve) and standard (blue dotdashed curve) electron cuts. The black dashed curve corresponds to events rejected by the removed cuts. . . . . . . . . . . .

3.2 Reconstructed $\Lambda$ mass for events rejected when all electron cuts are applied, at backward kaon center of mass angles $-1.0<\cos \theta_{K}^{C M}<-0.5$ for different $W$ bins starting from $1.6 \mathrm{GeV}$ to $2.2 \mathrm{GeV}$ with $50 \mathrm{MeV}$ bins.

3.3 CC signal threshold cut using the number of photoelectrons. . . . . . . .

3.4 EC deposited energy vs. momentum distribution for electrons. The $E_{\text {sample }}$ in this plot is the deposited energy divided by the calorimeter sampling fraction. The right plot shows the $E_{\text {sample }} / p_{e}$ ratio for the electrons. $\ldots \ldots \ldots \ldots \ldots \ldots \ldots . \cdots \cdots$

3.5 Energy deposition in the EC outer layers versus the EC inner layers (left). The same distribution with the pion cut is given on the right plot. The characteristic pion behavior is visible in both plots. . . . . . . . .

3.6 z-vertex distributions for electrons as a function of $\phi_{e}$. The left plot is before the vertex corrections and the right plot is after the correction. The applied cuts are shown by yellow lines. . . . . . . . . . . . 
3.7 $y$ vs. $x$ distributions of electrons projected on the calorimeter surface for sectors 3 and 4 . The position distributions before fiducial cuts (top), after geometrical fiducial cuts (middle) and after EC fiducial cuts (bottom) are shown. . . . . . . . . . . . . . . . . .

3.8 The angular coverage $\left(\theta_{e} \cos \phi_{e}\right.$ vs. $\left.\theta_{e} \sin \phi_{e}\right)$ of electrons before (left) and after fiducial cuts (right) . . . . . . . . . . . . . . .

$3.9 \theta_{e}$ vs. $p_{e}$ for Sector $3 \ldots \ldots \ldots \ldots \ldots \ldots$ 48

3.10 Minimum $\Delta t$ vs. $p$ distributions for kaons and protons. a) and b) show the distributions without any cuts. c) and d) show the same distributions for kaons and protons after applying the $\Lambda$ missing-mass and $\pi$ missingmass cuts.

$3.11 z$-vertex distribution for kaons before and after corrections. The applied cuts are shown by arrows and yellow lines. . . . . . . . . . . . .

3.12 Kaon $\theta_{K}$ vs. $\phi_{K}$ distributions for all sectors (top) and for the Sector 1 alone (bottom) before (left) and after (right) the fiducial cuts. . . . . . . 54

3.13 Kaon $\theta_{K}$ vs. $p_{K}$ for Sector $3 . \ldots \ldots \ldots \ldots$

3.14 Hadron mass vs. SC paddle number for each sector. Bad paddles can be identified from these plots. . . . . . . . . . . . . . . .

3.15 $\Lambda$ missing mass distributions a) before any cuts, b) after $p$ presence requirement. . . . . . . . . . . . . . .

3.16 a) Reconstructed meson missing mass squared vs. baryon missing mass correlation, b) Meson missing mass squared distribution. The red lines show the applied cuts on $\pi$ missing mass squared. c) baryon missing mass distribution after applying $\pi$ missing-mass-squared cut. The red lines in this plot show the missing mass range over which the background subtracted yields are integrated for the final $\Lambda$ sample selection. . . . . .

4.1 Kinematic distributions a) $Q^{2}$ vs. $W$, b) $Q^{2}$ vs. $\cos \theta_{K}^{C M}$, and c) $\phi_{K}^{C M}$ vs.

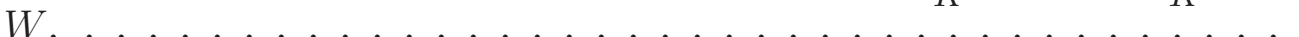

4.2 Electron $d p$ vs. $\phi_{e}$ distributions for all sectors before (top) and after (bottom) corrections. . . . . . . . . . . . . . . . . .

4.3 Electron hbprof distributions of $d p$ vs. $\phi_{e}$ for all sectors before (top) and after (bottom) corrections. Note the different $d p$ axis scales in the before and after plots. . . . . . . . . . . . . . . . . 
4.4 Electron $d p / p$ vs. $p_{e}$ distributions before (top) and after (bottom) corrections. The distributions are centered at zero after applying the momentum corrections. The gaps in these plots are the result of the removed SC paddles. . . . . . . . . . . . . . . . .

4.5 Hadron $d p / p$ vs. $p_{h}$ distributions before (left) and after (right) the hadron momentum corrections. The sharp transitions in these plots are the result of the elastic events. The gaps are the result of the removed SC paddles.

4.6 Sample fit of a $\Lambda \mathrm{MC}$ template. A Gaussian plus an asymmetrical Lorentzian on both sides of the Gaussian centroid was fit to the $\Lambda$ peak. . . . . . 71

4.7 $\Delta t$ vs. $p$ distributions for kaons with misidentified pion and proton bands overlayed. . . . . . . . . . . . . . . . .

4.8 Pion background templates for different $W$ bins for $0.8<\cos \theta_{K}^{C M}<1$. Plots show the distributions for $p$ 's moving both along and opposite to the longitudinal quantization axis. . . . . . . . . . . . . . . .

4.9 Pion background template for $p$ 's moving along the normal quantization axis. The black curve is the smoothed template used for background subtraction . . . . . . . . . . . . . . . . . . . . .

4.10 Typical fits to $\Lambda$ missing mass histograms at different $W$ bins using Eq. 4.9. The histograms corresponding to forward and backward $p$ angles are fitted simultaneously. Note that the backward $p$ histograms are shifted by $1 \mathrm{GeV}$. The green curve corresponds to the $\Lambda$ peak, the red curve corresponds to $\Sigma^{0}$ peak, the purple curve is the background and the blue curve is the total fit function. . . . . . . . . . . . . . . . . . .

4.11 Reduced $\chi^{2}$ distributions for a) $0.6<\cos \theta_{K}^{C M}<0.8$ and b) $0.8<\cos \theta_{K}^{C M}<1$

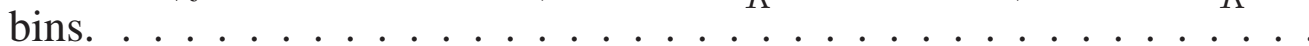




\section{LIST OF TABLES}

1.1 Summary of quantum numbers for basic set of baryons of Sakata model [2]. Here $B, z, J, P, T, S$ and $Y$ are the baryon number, electric charge, spin, parity, isospin, strangeness, and the hypercharge, respectively.

1.2 Table of pseudoscalar mesons constructed according to Sakata's constituent model [2]. . . . . . . . . . . . . . . . . . . .

1.3 Summary of quark quantum numbers [7]. Here $J, B, z, S, T$ and $T_{3}$ are the spin, baryon number, electric charge, strangeness, isospin and the third component of isospin, respectively. . . . . . . . . . . .

$1.4 N^{*}$ resonances below $2 \mathrm{GeV}$ listed by Particle Data Group (PDG) that couple to $K^{+} \Lambda$. The star rating is the PDG standard and the B.R. indicates the branching ratio $[7] \ldots \ldots \ldots \ldots \ldots$

1.5 Summary of quantum numbers of $K^{+}$and $\Lambda$ [7]. Here $J, B, z, S, T$ and $P$ are the Spin, Baryon number, electric charge, strangeness, isospin and parity respectively. $c \tau$ is the proper distance that the particle travels before decaying. . . . . . . . . . . . . . .

2.1 Hydrogen cryotarget parameters during the E1F run period.

3.1 Parameters used for electron geometrical fiducial cuts in Eq. 3.2. All angles are measured in degrees and momenta in GeV. . . . . . . . . . .

3.2 Parameters used for hadron geometrical fiducial cuts in Eq. 3.9. All angles are measured in degrees and momenta in $\mathrm{GeV} . . . . . . . .$.

3.3 List of removed $\mathrm{SC}$ paddles. . . . . . . . . . . . . . .

4.1 Binning for the polarization studies. $\ldots \ldots \ldots \ldots \ldots \ldots$

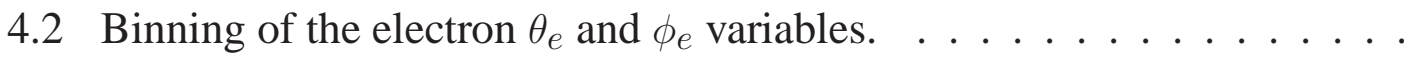

4.3 Summary of centroids and widths of $W$ distributions before and after the electron momentum corrections. The expected centroid values are supposed to be around the proton mass of $938 \mathrm{MeV} . \ldots \ldots \ldots$

4.4 Binning of the hadron $\theta_{h}$ and $\phi_{h}$ variables. . . . . . . . . . 67

4.5 Hyperon missing mass distributions for each sector before and after momentum corrections. Summary of centroids and widths after each set of

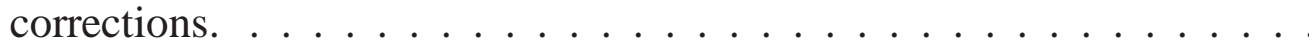


4.6 Hyperon missing mass distributions for each $\theta_{e}$ bin. Summary of centroids and widths after each set of corrections. . . . . . . . . . . . 69

4.7 $\Lambda$ missing mass distributions for each $W$ bin. Summary of centroids and widths after each set of corrections. . . . . . . . . . . . . 70

4.8 Binning for the $Q^{2}$ dependence studies. . . . . . . . . . . . . . . 87

5.1 Summary of the systematic uncertainties. The total systematic uncertainty, assigned for each $\cos \theta_{K}^{C M}$ bin is calculated as a sum of quadratures of the individual contributions. . . . . . . . . . . . . . . . 104

B.1 $\theta_{e}-p_{e}$ nominal cuts for electrons. . . . . . . . . . . . . . . . 117

B.2 $\theta_{h}-p_{h}$ nominal cuts for hadrons. . . . . . . . . . . . . 117

D.1 $P_{N}$ polarization results binned in $\cos \theta_{K}^{C M}$ and $W$ bins. The listed are only the statistical uncertainties. . . . . . . . . . . . . . . . . 120

D.2 $P_{N}$ polarization results binned in $\cos \theta_{K}^{C M}$ and $W$ bins. The listed are only the statistical uncertainties. . . . . . . . . . . . . . . . . . 121

D.3 $P_{L}$ polarization results binned in $\cos \theta_{K}^{C M}$ and $W$ bins. The listed are only the statistical uncertainties. . . . . . . . . . . . . . . . . 122

D.4 $P_{L}$ polarization results binned in $\cos \theta_{K}^{C M}$ and $W$ bins. The listed are only the statistical uncertainties. . . . . . . . . . . . . . . . 123

D.5 $P_{T}$ polarization results binned in $\cos \theta_{K}^{C M}$ and $W$ bins. The listed are only the statistical uncertainties. . . . . . . . . . . . . . . . . . 124

D.6 $P_{T}$ polarization results binned in $\cos \theta_{K}^{C M}$ and $W$ bins. The listed are only the statistical uncertainties. . . . . . . . . . . . . . . . . 125 


\section{CHAPTER 1}

\section{Introduction}

\subsection{Historical Overview}

Particle physics studies the internal structure of matter, investigating the properties of so-called elementary particles that make up matter and their interactions. But we must note that it was not an easy task to define what "elementary" means. The definition was constantly changing throughout the stages of development of particle physics. At every stage the elementary signified the smallest possible particle of matter that could not be further subdivided.

At early stages, in the absence of accelerators, cosmic rays were the only source from which the scientists could gather information about the properties of elementary particles and their interactions. By the end of 1949 several elementary particles

were discovered, among which were the electron $e^{-}$(1897), photon $\gamma(1900)$, proton $p$ (1919), neutron $n$ (1932), as well as $\mu^{ \pm}$(1938), $\pi^{ \pm}$(1947) and $K^{ \pm}$(1949). Each one of these particles was assigned quantum numbers such as electric charge, baryon number, spin, parity, strangeness etc., to describe their interaction properties. Conservation of quantum numbers in nuclear reactions was assumed to be a universal law. It was also known that the interactions are governed by four fundamental forces of nature: strong, electromagnetic, weak and gravitational.

By 1949 scientists already recognized that there were more known particles than quantum numbers, and some of them have very similar properties. This similarity motivated the first attempts to classify the known particles. One of the first attempts to classify the particles was made by Fermi and Yang in 1949, who chose $p$ and $n$ and their antiparticles $\bar{p}$ and $\bar{n}$ to be the basic elementary particles and tried to construct all other known particles by different combinations of these four. The Fermi and Yang model obviously failed when applied to strange particles. 


\begin{tabular}{|r|r|r|r|r|r|r|r|r|}
\hline PARTICLE & $m(\mathrm{MeV})$ & $B$ & $z$ & $J(h)$ & $P$ & $T$ & $S$ & $Y=S+B$ \\
\hline$p$ & 938.3 & +1 & +1 & $1 / 2$ & +1 & $1 / 2$ & 0 & +1 \\
\hline$n$ & 939.6 & +1 & 0 & $1 / 2$ & +1 & $1 / 2$ & 0 & +1 \\
\hline$\Lambda$ & 1115.6 & +1 & 0 & $1 / 2$ & +1 & 0 & -1 & 0 \\
\hline $\bar{p}$ & 938.3 & -1 & -1 & $1 / 2$ & -1 & $1 / 2$ & 0 & -1 \\
\hline $\bar{n}$ & 939.6 & -1 & 0 & $1 / 2$ & -1 & $1 / 2$ & 0 & -1 \\
\hline$\Lambda$ & 1115.6 & -1 & 0 & $1 / 2$ & -1 & 0 & +1 & 0 \\
\hline
\end{tabular}

Table 1.1: Summary of quantum numbers for basic set of baryons of Sakata model [2]. Here $B, z, J, P, T, S$ and $Y$ are the baryon number, electric charge, spin, parity, isospin, strangeness, and the hypercharge, respectively.

In 1951, track analysis of cosmic ray interactions revealed $V$-shape tracks that corresponded to a neutral particle decaying at rest into two charged particles. The detailed analysis demonstrated that the decay products were $p$ and $\pi^{-}$. So the newly discovered particle was a baryon that was a little heavier than the nucleons. The new particle was named $\Lambda$. The problem with $\Lambda$ particle was that it was produced in a strong interaction but the decay time was around $10^{-10} \mathrm{~s}$ which is typical for weak decay. This property was a characteristic of strange particles. So the newly discovered particle was a strange baryon.

In 1956 Sakata $[1,2,3]$ proposed an alternative model of particle classification. As a basic set he suggested taking the $\Lambda$ in addition to $p$ and $n$ and their antiparticles, in order for it to be possible to construct all known baryons as well as all strange particles. The members of the basic set should also have half-integer spins in order for it to be possible to construct the states with integer as well as half-integer spins. Table 1.1 summarizes the quantum numbers and masses of these baryons. The choice of baryons for the model was influenced by relative similarity of the particle masses (Table 1.1 [2]), which ensures that at very small distances, their strong interactions are approximately the same. When applied to mesons, it is obvious that the combination should be a baryon-antibaryon pair in order to ensure $B=0$. In the Sakata model it is shown that from three baryons and their antiparticles it is possible to construct nine baryon-antibaryon pair combinations: a unitary octet and a unitary singlet. Table 1.2 summarizes these nine combinations for pseudoscalar mesons. The six off-diagonal elements of Table 1.2 


\begin{tabular}{|rr|r|r|r|}
\hline ANTIPARTICLE & PARTICLE & $p$ & $n$ & $\Lambda$ \\
\hline $\bar{p}$ & $\bar{p} p$ & $\pi^{-}$ & $K^{-}$ \\
\hline $\bar{n}$ & $\pi^{+}$ & $\bar{n} n$ & $K^{0}$ \\
\hline $\bar{\Lambda}$ & $K^{+}$ & $K^{0}$ & $\bar{\Lambda} \Lambda$ \\
\hline
\end{tabular}

Table 1.2: Table of pseudoscalar mesons constructed according to Sakata's constituent model [2].

are identified with corresponding mesons. Three more combinations can be constructed from the linear combinations of the diagonal elements of Table 1.2. The first is $\pi^{0}$ with combination $(\bar{p} p-\bar{n} n) / \sqrt{2}$ and isospin $T=1$. The second combination is $(\bar{p} p+\bar{n} n-2 \bar{\Lambda} \Lambda) / \sqrt{6}$ with isospin $T=0$ corresponds to $\eta$. And the last combination is $(\bar{p} p+\bar{n} n+\bar{\Lambda} \Lambda) / \sqrt{3}$, fully symmetric under $p, n$ and $\Lambda$ exchange and forms a unitary singlet. The last combination later on turned out to be the $\eta \prime(958)$ meson. According to Sakata model, pions and kaons belong to the same unitary octet which was surprising because of the relatively large mass difference between the pions and kaons. But when these particles were viewed as bound states and the binding energy was calculated, the relative binding energy ratio turned out to be $0.1 \ll 1$. This statement can be proved for all eight members of the unitary octet [2]. The defects of Sakata model become noticeable when applied to baryons. The three baryon combinations must be excluded because no baryons with $B=3$ are observed in nature. The conclusion was that the basic set could not be $p, n$, and $\Lambda$.

Although the Sakata model does not describe all known hadron properties, it serves a nice introduction to subsequent attempts of hadron classification. The new models also used the same basic assumption made in Sakata model that the hadrons with the same spin and parity can be grouped into multiplets. The most successful alternative model was suggested by Gell-Mann and Neeman in 1961 [4]. They assumed that the basic set of particles must have fractional baryon charge with $B=1 / 3$ and spin=1/2. The electric charge was calculated according to $z=T_{3}+\frac{B+S}{2}$ [2], which was also fractional. In 1964 Gell-Mann named these particles “quarks." In their "Eightfold Way” model [4] they constructed mesons from quark-antiquark pairs and baryons from three quarks. 


\begin{tabular}{|r|r|r|r|r|r|r|}
\hline QUARK & $J$ & $B$ & $z$ & $S$ & $T$ & $T_{3}$ \\
\hline$u$ & $1 / 2$ & $1 / 3$ & $+2 / 3$ & 0 & $1 / 2$ & $+1 / 2$ \\
\hline$d$ & $1 / 2$ & $1 / 3$ & $-1 / 3$ & 0 & $1 / 2$ & $-1 / 2$ \\
\hline$s$ & $1 / 2$ & $1 / 3$ & $-1 / 3$ & -1 & 0 & 0 \\
\hline$c$ & $1 / 2$ & $1 / 3$ & $+2 / 3$ & 0 & 0 & 0 \\
\hline$b$ & $1 / 2$ & $1 / 3$ & $-1 / 3$ & 0 & 0 & 0 \\
\hline$t$ & $1 / 2$ & $1 / 3$ & $+2 / 3$ & 0 & 0 & 0 \\
\hline
\end{tabular}

Table 1.3: Summary of quark quantum numbers [7]. Here $J, B, z, S, T$ and $T_{3}$ are the spin, baryon number, electric charge, strangeness, isospin and the third component of isospin, respectively.

When applied to baryons, combining three quarks gave the correct sets of multiplets that were observed in nature. Within the framework of this model, they were able to construct all baryons and mesons known at that time and even predict the existence of the $\Omega^{-}$. The strong objection to this model was the fact that it required three identical quark combinations in direct contradiction with the Pauli principle, which states that no two particles with the same quantum numbers can occupy the same spin state. The way out from this situation was given in the framework of Quantum Chromodynamics (QCD), which assigned "color" charge (red, green and blue) to quarks as an analog to electric charge in Quantum Electrodynamics (QED).

\subsection{Motivation}

At the present moment there are six known quarks. The quark quantum numbers are summarized in Table 1.3. The interaction between quarks is mediated by gluons, the gauge bosons of strong interactions. The QCD theory describes our current understanding of the interactions between the quarks and gluons. QCD predicts the existence of excited nucleon states, called $N^{*}$ resonances, some of which have been observed experimentally. These resonances form the QCD spectrum. According to some existing explanations, baryon excitations are the result of interquark interaction dynamics, or the presence of diquark clustering inside baryons. The causes of excitations are still under investigation. They are the driving force for most of the current Jefferson Laboratory 
experiments and also are the focus of the present analysis.

According to QCD, quarks can emit and absorb gluons, much the same way as a charged particle can emit and absorb photons according to the QED theory. The strength of these interactions is determined by so called coupling constants. Unlike QED, where the coupling constant $\alpha=1 / 137$ is independent of energy (for Jefferson Laboratory energies), the coupling constant of the strong interactions in QCD is pretty much energy dependent, and goes to zero as the interquark separation $r$ goes to zero [2]:

$$
g_{s}^{2}(r) \sim \frac{1}{\ln r^{-1}} .
$$

At very high energies the coupling constant of the strong interaction is very small, but at energies below $\sim 7 \mathrm{GeV}, g_{s} \gtrsim 1$. All calculations both in QED and QCD are performed using perturbation theory with the corresponding coupling constants used as expansion parameters. In QED calculations, every higher order term acquires an additional factor $\alpha^{2}$, therefore decreasing its contribution by a factor of $\alpha^{2} \sim \frac{1}{10000}$ at all energies. One can stop including terms once the desired precision is reached. At the present moment the experimental results and QED calculations agree up to ten significant digits. Perturbation theory works fine for QCD calculations at high energies as well. At low energies, however, this technique cannot be used because the contribution from every higher order term either increases or contributes with approximately the same weight $\left(g_{s} \sim 1\right)$ to the amplitude.

As an alternative, different quark models were developed for performing calculations in the low-energy region where perturbative QCD cannot be applied. One way to test the predictions of these models is to obtain the QCD spectrum, since it can be verified experimentally. One such model is the constituent quark model (CQM) proposed by Capstick and Roberts [5]. In CQM model, baryons are treated as three quark systems consisting of only valence quarks ( $u, d$ and $s$ ) with relativized wave functions. The CQM model is the relativized version of the ${ }^{3} P_{0}$ hadron decay model, which assumes that the hadron decay goes via production of quark-antiquark pairs with a quantum num- 


\begin{tabular}{|r|r|r|}
\hline \multicolumn{3}{|c|}{$N * \rightarrow K \Lambda$} \\
\hline State & Rating & B.R. $(K \Lambda)$ \\
\hline$N^{*}(1650) S_{11}$ & $* * * *$ & $3-11 \%$ \\
\hline$N^{*}(1675) D_{15}$ & $* * * *$ & $<1 \%$ \\
\hline$N^{*}(1680) F 15$ & $* * * *$ & - \\
\hline$N^{*}(1700) D_{13}$ & $* * *$ & $<3 \%$ \\
\hline$N^{*}(1710) P_{11}$ & $* * *$ & $5-25 \%$ \\
\hline$N^{*}(1720) P_{13}$ & $* * *$ & $1-15 \%$ \\
\hline$N^{*}(1900) P_{13}$ & $* *$ & $2.4 \%$ \\
\hline$N^{*}(1990) F_{17}$ & $* *$ & - \\
\hline$N^{*}(2000) F_{15}$ & $* *$ & - \\
\hline
\end{tabular}

Table 1.4: $N^{*}$ resonances below $2 \mathrm{GeV}$ listed by Particle Data Group (PDG) that couple to $K^{+} \Lambda$. The star rating is the PDG standard and the B.R. indicates the branching ratio [7]

ber $J^{P C}=0^{++}$(consistent with vacuum quantum numbers). These quantum numbers correspond to the ${ }^{3} P_{0}$ state, hence the name of the model.

The predictions of these models introduce the so called missing resonance problem. The problem is that the models predict about four times more resonance states than have been experimentally detected. One of the explanations is that the formation channel plays a significant role in resonance creation. Most of the existing experimental results are for the $\pi N \rightarrow N^{*} \rightarrow \pi N$ reactions. But calculations show that not all resonances can be created via this channel. To fully understand the production and decays of excited baryon states, other reaction channels must be explored. Recent experiments revealed that some $N^{*}$ resonances can be created via $\gamma p$ photoproduction and $e p$ electroproduction.

For this analysis we have chosen the $e+p \rightarrow e^{\prime}+K^{+}+\Lambda$ reaction. The $K^{+} \Lambda$ production mechanism is treated as a two step process: first the nonstrange baryon resonance is formed in the $s$-channel, then it decays into the final $K^{+} \Lambda$ state. Table 1.4 summarizes the list of known $N^{*}$ baryons that can couple to the $K^{+} \Lambda$ channel. Although this final state has a low cross section, the two-body decay is kinematically more favorable for states below $2 \mathrm{GeV}$, because of the relatively higher masses of the particles involved, as compared to the multipion final states. Studying this final state becomes more advantageous, since most of the missing resonances are predicted to have masses below $2 \mathrm{GeV}$. 


\begin{tabular}{|r|r|r|r|r|r|r|r|r|r|}
\hline PARTICLE & Decay Mode & Mass $(\mathrm{MeV})$ & $J$ & $B$ & $z$ & $S$ & $T$ & $P$ & $c \tau$ \\
\hline$K^{+}(u \bar{s})$ & $\mu^{+} \nu_{\mu}(63 \%)$ & 493.677 & 0 & 0 & +1 & +1 & $1 / 2$ & -1 & $3.712 \mathrm{~m}$ \\
\hline$\Lambda^{0}(u d s)$ & $p \pi^{-}(63 \%)$ & 1115.683 & $1 / 2$ & 1 & 0 & -1 & 0 & +1 & $7.89 \mathrm{~cm}$ \\
\hline
\end{tabular}

Table 1.5: Summary of quantum numbers of $K^{+}$and $\Lambda$ [7]. Here $J, B, z, S, T$ and $P$ are the Spin, Baryon number, electric charge, strangeness, isospin and parity respectively. $c \tau$ is the proper distance that the particle travels before decaying.

The masses and full quantum numbers of both $K^{+}$and $\Lambda$ are summarized in Table 1.5.

The $K^{+} \Lambda$ channel is relatively easy to detect with the CLAS detector because of its large acceptance. A detailed description is given in Chapter 2.1. The $K^{+}$lives long enough to be detected directly, while the $\Lambda$ travels, on average, only $\sim 8 \mathrm{~cm}$ before decaying. Experimentally, the $\Lambda$ can be identified either via detecting both decay products that traverse the CLAS detector then reconstructing the invariant mass or by reconstructing the hyperon missing mass spectrum. The later method is used for present analysis and is discussed in detail in Section 3.3. Because of the parity violating decay of $\Lambda$, this channel is also easy to analyze for polarization by looking at the angular distributions of one of the decay products ( $p$ from $p \pi^{-}$decay mode for present analysis). Note that $K^{+}$and $\Lambda$ are produced in the strong interaction which conserves isospin. The isospin conservation prevents $\Delta$ resonances from decaying into $K^{+} \Lambda$, thus playing the role of a filter.

The strange quark plays an important role in understanding the strong interactions of the nucleons. The investigation of strangeness production in both photo- and electroproduction reactions has been carried out since the 1950s, but as of today, there is no comprehensive model describing the reaction mechanism. The present analysis is part of a larger program, carried out at Jefferson Lab, to determine cross sections and polarization observables in kaon photo- and electroproduction, with a final goal of developing a comprehensive model of the strangeness production process. The cross sections and the polarization observables can be expressed in terms of response functions according to the framework of Ref. [6]. In order to have a model-independent description of pseudoscalar meson production, a total of 36 independent response functions 
need to be measured in single- and double-polarization experiments as summarized in Table A.1 [6]. Some of these observables have already been measured and are discussed in Section 1.6. Polarization observables possess a strong discriminatory power that can be used for distinguishing between different theoretical models and their variants when trying to describe the underlying strangeness production mechanism, for which the differential cross sections alone has proven to be insufficient. The results of the current analysis, when added to the world database, will help to constrain model parameters of strangeness production. Ultimately, a full partial wave analysis will provide information about $N^{*}$ resonances involved in the production process. The results can confirm or reject the existence of weakly established or missing $N^{*}$ resonances.

\subsection{Physics Variables and Formalism}

The kinematics of the $e+p \rightarrow e^{\prime}+K^{+}+\Lambda$ reaction is shown in Fig. 1.1. The process of electron scattering off of a nucleon is mediated by the exchange of a virtual photon. As a result of this interaction two strange particles $K^{+}(u \bar{s})$ and $\Lambda(u d s)$ are produced in the final state. This process is known as strangeness electroproduction. The virtual photon is characterized by two Lorentz invariant variables: the transferred energy, $\nu$ and the transferred four-momentum squared, $Q^{2}$ :

$$
\begin{aligned}
\nu & =E_{i}-E_{f} \\
Q^{2} & =-\left(p_{e}-p_{e^{\prime}}\right)^{2}=4 E_{i} E_{f} \sin ^{2}\left(\frac{\theta_{e}}{2}\right),
\end{aligned}
$$

where $E_{i}$ and $E_{f}$ are the initial and final electron energies in the laboratory frame. The $p_{e}$ and $p_{e^{\prime}}$ are the initial and final four-momenta of the electron, respectively, and $\theta_{e}$ is the electron scattering angle in the laboratory frame. The virtual photons, denoted as $\gamma^{*}$, possess both longitudinal and transverse polarization unlike real photons, which 


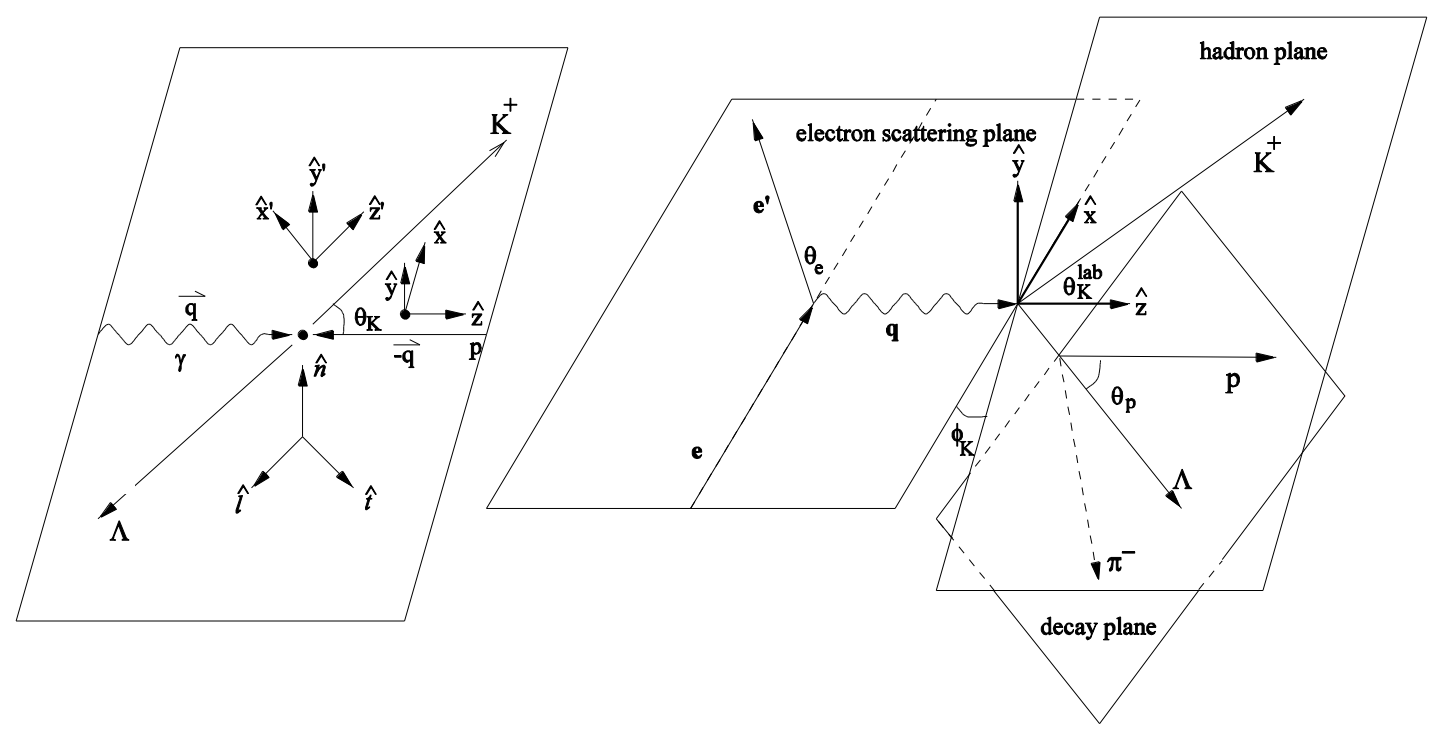

Figure 1.1: Kinematics for $K^{+} \Lambda$ electroproduction showing angles and polarization axes in the c.m. (left) and lab (right) reference frames.

only possess transverse polarization. The $\gamma^{*}$ polarization components are given by:

$$
\begin{aligned}
\epsilon & =\frac{1}{1+2\left(1+\frac{\nu^{2}}{Q^{2}}\right) \tan ^{2}\left(\frac{\theta_{e}}{2}\right)}, \\
\epsilon_{L} & =\frac{Q^{2}}{\nu^{2}} \epsilon .
\end{aligned}
$$

Another important set of Lorentz invariant variables are the so called Mandelstam variables, which manifest the four-momentum conservation in the scattering process:

$$
\begin{aligned}
s & =\left(q+p_{t}\right)^{2}=\left(p_{K}+p_{\Lambda}\right)^{2}, \\
t & =\left(q-p_{K}\right)^{2}=\left(p_{t}-p_{\Lambda}\right)^{2}, \\
u & =\left(q-p_{\Lambda}\right)^{2}=\left(p_{t}-p_{K}\right)^{2} .
\end{aligned}
$$

In these equations $q, p_{t}, p_{K}$, and $p_{\Lambda}$ are the four momenta of the virtual photon, target proton, kaon, and $\Lambda$, respectively. Mandelstam variables define the corresponding $s, t$, and $u$-channels of the scattering process shown in Fig. 1.2. Each channel corresponds to a Feynman diagram where the invariant mass squared of the intermediate or exchanged 


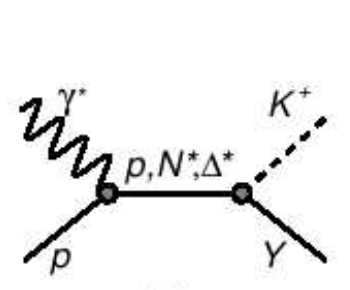

(a)

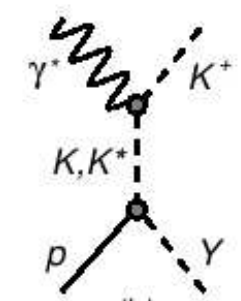

(b)

t-channel

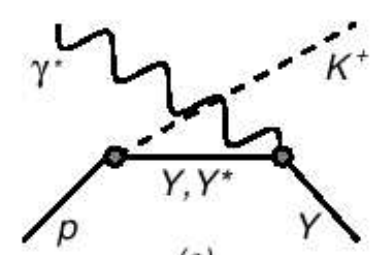

(c)

u-channel

Figure 1.2: $e p \rightarrow K Y$ reaction channel diagrams.

particle is equal to $s, t$, and $u$, respectively. The intermediate hadronic-state energy $W=\sqrt{s}$ is:

$$
W^{2}=s=M_{p}^{2}+2 M_{p} \nu-Q^{2} .
$$

The target proton is stationary in the laboratory frame, so its four-momentum is $\left(M_{p}, 0\right)$, where $M_{p}$ is the mass of the proton.

The present analysis employs the missing-mass technique to identify the final state of interest. The missing-mass technique uses energy and momentum conservation laws to reconstruct the mass of the undetected (missing) particle. Since $\Lambda$ cannot be detected directly because of its short lifetime, it is identified by its reconstructed mass. In the $e p \rightarrow e^{\prime} K^{+} X$ reaction the missing mass is defined as:

$$
M_{X}^{2}=\left(\left(p_{e}+p_{t}\right)-\left(p_{e^{\prime}}+p_{K}\right)\right)^{2} .
$$

The $M_{X}^{2}$ missing mass calculated from Eq. 1.8 for the $p\left(e, e^{\prime} K^{+}\right) \Lambda$ final state should be consistent with the $\Lambda$ hyperon mass. In the same way, the missing mass for the $e p \rightarrow e^{\prime} K^{+} p X$ reaction can be calculated by:

$$
M_{X}^{2}=\left(\left(p_{e}+p_{t}\right)-\left(p_{e^{\prime}}+p_{K}+p_{p}\right)\right)^{2},
$$

where $p_{p}$ is the four-momentum of the detected proton that comes from the hyperon decay. In this analysis the $M_{X}^{2}$, calculated by Eq. 1.9, is constrained to be consistent 
with the pion mass squared.

The reaction kinematics are uniquely defined by the set of four variables $\left(Q^{2}, W\right.$, $\left.\cos \theta_{K}^{C M}, \phi_{K}\right)$, where $\theta_{K}^{C M}$ is the kaon angle in the CM frame defined in Fig. 1.1. $\phi_{K}$ is the relative angle between the electron-scattering and the hadron-production planes.

The $K^{+} \Lambda$ electroproduction cross section in the most general form can be expressed as a product of the virtual photon flux and the photo absorption cross section [8]:

$$
\frac{d^{3} \sigma}{d \Omega_{E^{\prime}} d \Omega_{K} d E^{\prime}}=\Gamma \frac{d \sigma_{\nu}}{d \Omega_{K}}
$$

where

$$
\Gamma=\frac{\alpha}{4 \pi} \frac{W}{M_{p}^{2} E^{2}}\left(W^{2}-M_{p}^{2}\right)\left[\frac{1}{Q^{2}(1-\epsilon)}\right] .
$$

The photo absorption cross section in terms of the response functions $R_{i}^{\beta \alpha}$ is given using the notations of Ref. [6] as:

$$
\begin{aligned}
\frac{d \sigma_{\nu}}{d \Omega_{K}} & =K S_{\alpha} S_{\beta}\left[R_{T}^{\beta \alpha}+\epsilon_{L} R_{L}^{\beta \alpha}+\sqrt{2 \epsilon_{L}(1+\epsilon)}\left({ }^{c} R_{T L}^{\beta \alpha} \cos \phi_{K}+{ }^{s} R_{T L}^{\beta \alpha} \sin \phi_{K}\right)\right. \\
& +\epsilon\left({ }^{c} R_{T T}^{\beta \alpha} \cos 2 \phi_{K}+{ }^{s} R_{T T}^{\beta \alpha} \sin 2 \phi_{K}\right) \\
& \left.+h \sqrt{2 \epsilon_{L}(1-\epsilon)}\left({ }^{c} R_{T L^{\prime}}^{\beta \alpha} \cos \phi_{K}+{ }^{s} R_{T L^{\prime}}^{\beta \alpha} \sin \phi_{K}\right)+h \sqrt{1-\epsilon^{2}} R_{T T^{\prime}}^{\beta \alpha}\right]
\end{aligned}
$$

In this expression, the kinematic factor $K=\frac{\left|\mathbf{p}_{\mathbf{K}}\right|}{k_{\gamma}^{C M}}$ is the ratio of the kaon center-of-mass and virtual photon momenta and $h$ is the electron-beam helicity. The superscripts $\alpha$ and $\beta$ refer to the target and $\Lambda$ polarizations, respectively, where a sum over $\alpha$ and $\beta$ is implied. The $c$ and $s$ superscripts on the response functions refer to the cosine or sine terms they accompany. Table A.1 summarizes which response functions survive for different polarizations.

The spin-projection operators are defined as

$$
\begin{aligned}
& S_{\alpha}=(1, \mathbf{S}), \\
& S_{\beta}=\left(1, \mathbf{S}^{\prime}\right),
\end{aligned}
$$


with

$$
\begin{aligned}
\mathbf{S} & =\left(\hat{S}_{x}, \hat{S}_{y}, \hat{S}_{z}\right), \\
\mathbf{S}^{\prime} & =\left(\hat{S}_{x^{\prime}}, \hat{S}_{y^{\prime}}, \hat{S}_{z^{\prime}}\right) .
\end{aligned}
$$

The unprimed-coordinate system $\mathbf{S}$ is associated with the electron-scattering plane. It is defined with the $\hat{z}$ axis along the virtual photon momentum vector $\vec{q}, \hat{y}$ is normal to the electron-scattering plane, and $\hat{x}=\hat{y} \times \hat{z}$. The primed-coordinate system $\mathbf{S}^{\prime}$ is associated with the hadron-plane coordinates and is defined so that $\hat{z}^{\prime}$ is along the kaon momentum vector $\vec{p}_{K}$, with $\hat{y}^{\prime}$ normal to the hadron production plane, and $\hat{x}^{\prime}=\hat{y}^{\prime} \times \hat{z}^{\prime}$.

In the simplest case nothing is polarized, so the contributions from the beam, target and recoil polarization vanish, and equation Eq. 1.12 reduces to

$$
\sigma_{0} \equiv\left(\frac{d \sigma_{\nu}}{d \Omega_{K}}\right)^{00}=K\left[R_{T}^{00}+\epsilon_{L} R_{L}^{00}+\sqrt{2 \epsilon_{L}(1+\epsilon)} R_{T L}^{00} \cos \phi_{K}+\epsilon R_{T T}^{00} \cos 2 \phi_{K}\right]
$$

so that $K R_{i}^{00}=\sigma_{i}$ are the usual unpolarized cross-section components.

During this experiment, a polarized electron beam was incident upon an unpolarized target proton, producing a polarized recoil hyperon. For this case, Eq. 1.12 becomes

$$
\frac{d \sigma_{\nu}}{d \Omega_{K}}=\sigma_{0}\left(1+h A_{T L^{\prime}}+P_{x^{\prime}} \hat{S}_{x^{\prime}}+P_{y^{\prime}} \hat{S}_{y^{\prime}}+P_{z^{\prime}} \hat{S}_{z^{\prime}}\right)
$$

where

$$
A_{T L^{\prime}}=\frac{K}{\sigma_{0}} \sqrt{2 \epsilon_{L}(1-\epsilon)} R_{T L^{\prime}}^{00} \sin \phi_{K}
$$

describes the electron beam spin asymmetry. The $P_{j^{\prime}}$ terms describe the hyperon polarizations. Each component of the polarization by itself can be expressed as a sum of the beam-helicity independent (induced polarization) and helicity-dependent (transferred polarization) terms $P_{j^{\prime}}=P_{j^{\prime}}^{0}+h P_{j^{\prime}}^{\prime}$, where $P_{j^{\prime}}^{0}$ denotes the induced polarization of the $\Lambda$, and $P_{j^{\prime}}^{\prime}$ corresponds to the transferred polarization. Both polarization components in 
the primed coordinate system can be expressed in terms of the response functions as:

$$
\begin{aligned}
P_{x^{\prime}}^{0} & =\frac{K}{\sigma_{0}}\left(\sqrt{2 \epsilon_{L}(1+\epsilon)} R_{T L}^{x^{\prime} 0} \sin \phi_{K}+\epsilon R_{T T}^{x^{\prime} 0} \sin 2 \phi_{K}\right) \\
P_{y^{\prime}}^{0} & =\frac{K}{\sigma_{0}}\left(R_{T}^{y^{\prime} 0}+\epsilon_{L} R_{L}^{y^{\prime} 0}+\sqrt{2 \epsilon_{L}(1+\epsilon)} R_{T L}^{y^{\prime} 0} \cos \phi_{K}+\epsilon R_{T T}^{y^{\prime} 0} \cos 2 \phi_{K}\right) \\
P_{z^{\prime}}^{0} & =\frac{K}{\sigma_{0}}\left(\sqrt{2 \epsilon_{L}(1+\epsilon)} R_{T L}^{z^{\prime} 0} \sin \phi_{K}+\epsilon R_{T T}^{z^{\prime} 0} \sin 2 \phi_{K}\right) \\
P_{x^{\prime}}^{\prime} & =\frac{K}{\sigma_{0}}\left(\sqrt{2 \epsilon_{L}(1-\epsilon)} R_{T L^{\prime}}^{x^{\prime} 0} \cos \phi_{K}+\sqrt{1-\epsilon^{2}} R_{T T^{\prime}}^{x^{\prime} 0}\right) \\
P_{y^{\prime}}^{\prime} & =\frac{K}{\sigma_{0}} \sqrt{2 \epsilon_{L}(1-\epsilon)} R_{T L^{\prime}}^{y^{\prime} 0} \sin \phi_{K} \\
P_{z^{\prime}}^{\prime} & =\frac{K}{\sigma_{0}}\left(\sqrt{2 \epsilon_{L}(1-\epsilon)} R_{T L^{\prime}}^{z^{\prime} 0} \cos \phi_{K}+\sqrt{1-\epsilon^{2}} R_{T T^{\prime}}^{z^{\prime} 0}\right) .
\end{aligned}
$$

Eq. 1.14 can be integrated over $\phi_{K}$ angle. The experimental purpose of the integration is to improve the statistics and allow fine binning in $W$ and $\cos \theta_{K}^{C M}$ variables. First we need to define the transformation that relates the coordinates associated with these planes. The transformation from the primed coordinate system to the unprimed coordinate system is achieved by simple rotations, first by $\theta_{K}$ about $\hat{y}^{\prime}$, followed by $\phi_{K}$ about $\hat{z}^{\prime}$. The rotation matrix that relates the coordinates is

$$
R=\left(\begin{array}{ccc}
\cos \theta_{K} \cos \phi_{K} & -\sin \phi_{K} & \sin \theta_{K} \cos \phi_{K} \\
\cos \theta_{K} \sin \phi_{K} & \cos \phi_{K} & \sin \theta_{K} \sin \phi_{K} \\
-\sin \theta_{K} & 0 & \cos \theta_{K}
\end{array}\right)
$$

We can define the spin-projection operator in the hadron plane in terms of that of the electron plane using this transformation : $\hat{S}_{j^{\prime}}=R^{-1} \hat{S}_{j}$. Using these relationships for $\hat{S}_{j^{\prime}}$, the cross section can be rewritten as:

$$
\frac{d \sigma_{\nu}}{d \Omega_{K}}=\sigma_{0}\left(1+h A_{T L^{\prime}}+P_{x} \hat{S_{x}}+P_{y} \hat{S}_{y}+P_{z} \hat{S_{z}}\right)
$$


where $P_{j}=P_{j}^{0}+h P_{j}^{\prime}$ with

$$
\begin{aligned}
P_{x}^{0} & =P_{x^{\prime}}^{0} \cos \theta_{K} \cos \phi_{K}+P_{y^{\prime}}^{0}\left(-\sin \phi_{K}\right)+P_{z^{\prime}}^{0} \sin \theta_{K} \cos \phi_{K} \\
P_{y}^{0} & =P_{x^{\prime}}^{0} \cos \theta_{K} \sin \phi_{K}+P_{y^{\prime}}^{0} \cos \phi_{K}+P_{z^{\prime}}^{0} \sin \theta_{K} \sin \phi_{K} \\
P_{z}^{0} & =P_{x^{\prime}}^{0}\left(-\sin \theta_{K}\right)+P_{z^{\prime}}^{0} \cos \theta_{K} \\
P_{x}^{\prime} & =P_{x^{\prime}}^{\prime} \cos \theta_{K} \cos \phi_{K}+P_{y^{\prime}}^{\prime}\left(-\sin \phi_{K}\right)+P_{z^{\prime}}^{\prime} \sin \theta_{K} \cos \phi_{K} \\
P_{y}^{\prime} & =P_{x^{\prime}}^{\prime} \cos \theta_{K} \sin \phi_{K}+P_{y^{\prime}}^{\prime} \cos \phi_{K}+P_{z^{\prime}}^{\prime} \sin \theta_{K} \sin \phi_{K} \\
P_{z}^{\prime} & =P_{x^{\prime}}^{\prime}\left(-\sin \theta_{K}\right)+P_{z^{\prime}}^{\prime} \cos \theta_{K} .
\end{aligned}
$$

These are now the observed induced $\left(P_{j}^{0}\right)$ and transferred $\left(P_{j}^{\prime}\right)$ polarizations of the $\Lambda$ measured with respect to the electron-plane coordinate system.

The integration over $\phi_{K}$ from 0 to $2 \pi$ greatly simplifies the cross section expression:

$$
\int_{0}^{2 \pi} \frac{d \sigma_{\nu}}{d \Omega_{K}} d \phi_{k}=\left(\int \sigma_{0}\right)\left(1+\mathcal{P}_{x} S_{x}+\mathcal{P}_{y} S_{y}+\mathcal{P}_{z} S_{z}\right)
$$

where

$$
\int \sigma_{0}=2 \pi K\left(R_{T}^{00}+\epsilon_{L} R_{L}^{00}\right)
$$

and

$$
\mathcal{P}_{j}=\mathcal{P}_{j}^{0}+h \mathcal{P}_{j}^{\prime}
$$

where $\mathcal{P}_{j}$ s are $\phi_{K}$-integrated polarization components. The individual $\mathcal{P}_{j}$ s are:

$$
\begin{aligned}
\mathcal{P}_{x}^{0} & =0 \\
\mathcal{P}_{y}^{0} & =\pi \sqrt{2 \epsilon_{L}(1+\epsilon)} \frac{K}{\sigma_{0}}\left(R_{T L}^{x^{\prime} 0} \cos \theta_{K}+R_{T L}^{y^{\prime} 0}+R_{T L}^{z^{\prime} 0} \sin \theta_{K}\right) \\
\mathcal{P}_{z}^{0} & =0 \\
\mathcal{P}_{x}^{\prime} & =\pi \sqrt{2 \epsilon_{L}(1-\epsilon)} \frac{K}{\sigma_{0}}\left(R_{T L^{\prime}}^{x^{\prime} 0} \cos \theta_{K}-R_{T L^{\prime}}^{y^{\prime} 0}+R_{T L^{\prime}}^{z^{\prime} 0} \sin \theta_{K}\right) \\
\mathcal{P}_{y}^{\prime} & =0 \\
\mathcal{P}_{z}^{\prime} & =2 \pi \sqrt{1-\epsilon^{2}} \frac{K}{\sigma_{0}}\left(-R_{T T^{\prime}}^{x^{\prime} 0} \sin \theta_{K}+R_{T T^{\prime}}^{z^{\prime} 0} \cos \theta_{K}\right) .
\end{aligned}
$$


The $\phi_{K}$ integrated $\mathcal{P}_{j^{\prime}}$ components in the primed coordinate system are given by:

$$
\begin{aligned}
\mathcal{P}_{x^{\prime}}^{0} & =0 \\
\mathcal{P}_{y^{\prime}}^{0} & =\frac{K}{\sigma_{0}}\left(R_{T}^{y^{\prime} 0}+\epsilon_{L} R_{L}^{y^{\prime} 0}\right) \\
\mathcal{P}_{z^{\prime}}^{0} & =0 \\
\mathcal{P}_{x^{\prime}}^{\prime} & =\frac{K}{\sigma_{0}} \sqrt{1-\epsilon^{2}} R_{T T^{\prime}}^{x^{\prime} 0} \\
\mathcal{P}_{y^{\prime}}^{\prime} & =0 \\
\mathcal{P}_{z^{\prime}}^{\prime} & =\frac{K}{\sigma_{0}} \sqrt{1-\epsilon^{2}} R_{T T^{\prime}}^{z^{\prime} 0} .
\end{aligned}
$$

The coordinate system, $(\hat{t}, \hat{n}, \hat{l})$, that was used in this analysis, is defined with $\hat{l}$ along the $\Lambda$ momentum $\left(\hat{l}=-\hat{z}^{\prime}\right), \hat{n}$ normal to the hadron plane and $\hat{t}=-\hat{x}^{\prime}$. The polarization components in this system are given by:

$$
P_{t}=-P_{x^{\prime}} \quad P_{n}=P_{y^{\prime}} \quad P_{l}=-P_{z^{\prime}}
$$

The integration over $\phi_{K}$ gives:

$$
\begin{aligned}
\mathcal{P}_{t}^{0} & =0 \\
\mathcal{P}_{n}^{0} & =\frac{K}{\sigma_{0}}\left(R_{T}^{y^{\prime} 0}+\epsilon_{L} R_{L}^{y^{\prime} 0}\right) \\
\mathcal{P}_{l}^{0} & =0 \\
\mathcal{P}_{t}^{\prime} & =-\frac{K}{\sigma_{0}} \sqrt{1-\epsilon^{2}} R_{T T^{\prime}}^{x^{\prime} 0} \\
\mathcal{P}_{n}^{\prime} & =0 \\
\mathcal{P}_{l}^{\prime} & =-\frac{K}{\sigma_{0}} \sqrt{1-\epsilon^{2}} R_{T T^{\prime}}^{z^{\prime} 0}
\end{aligned}
$$

From Eq. 1.24 we see that only the normal component of the induced polarization survives the $\phi_{K}$ integration and only the in-plane components survive for the transferred part. 


\section{$1.4 \Lambda$ Polarization}

Although the $\Lambda$ is produced in a strong hadronization process it can only decay weakly. The reason for this is that $\Lambda$ is not heavy enough to decay into a nucleon and another strange meson in order to preserve strangeness, which necess arily has to be conserved in a strong process. The main decay modes of $\Lambda$ are $p \pi^{-}$and $n \pi^{0}$ with branching ratios of $64 \%$ and $36 \%$, respectively [7].

One can qualitatively show that the induced polarization can only have a non-zero component normal to the production plane, while the other two components must turn to zero. The $p\left(e, e^{\prime} K^{+} p\right) \pi^{-}$reaction is an electromagnetic interaction which conserves parity. The total cross section of the reaction must be invariant under parity transformation. The differential cross section of this interaction is given according to Eq. 1.14 for the case of a polarized electron beam, polarized recoil, and an unpolarized target proton. The beam spin asymmetry term will drop out after integration. Under parity transformation any in-plane component will change the sign. In order for the total cross section of the reaction to be invariant under parity transformation, the in-plane components must turn to zero. Recall that under the parity transformation in the production plane, the momenta $p_{\Lambda} \rightarrow-p_{\Lambda}$ and $p_{K^{+}} \rightarrow-p_{K^{+}}$, but $p_{\Lambda} \times p_{K^{+}}$does not change the sign, meaning that $p_{\Lambda} \times p_{K^{+}}$is invariant under the parity transformation. So, the $\Lambda$ spin is forced to be oriented either along or opposite to $p_{\Lambda} \times p_{K^{+}}$. On the other hand, $p_{\Lambda} \times p_{K^{+}}$is, by definition, perpendicular to the production plane. This qualitative argument regarding the induced polarization only works if the target is unpolarized. On the other hand, if the target is polarized, then $\Lambda$ induced polarization is not constraint to be in the normal direction but can also have in-plane components.

Parity violation is a general property of weak decays, which allows extraction of the $\Lambda$ polarization from the angular distribution of one of the decay products. The remainder of this section describes in detail the self-analyzing nature of the $\Lambda$ [3].

Fig. 1.3 shows definitions of the coordinate axes and directions of the $\Lambda$ decay products. The $z$-axis is defined along the $\Lambda$ spin direction in the $\Lambda$ rest frame. The total 


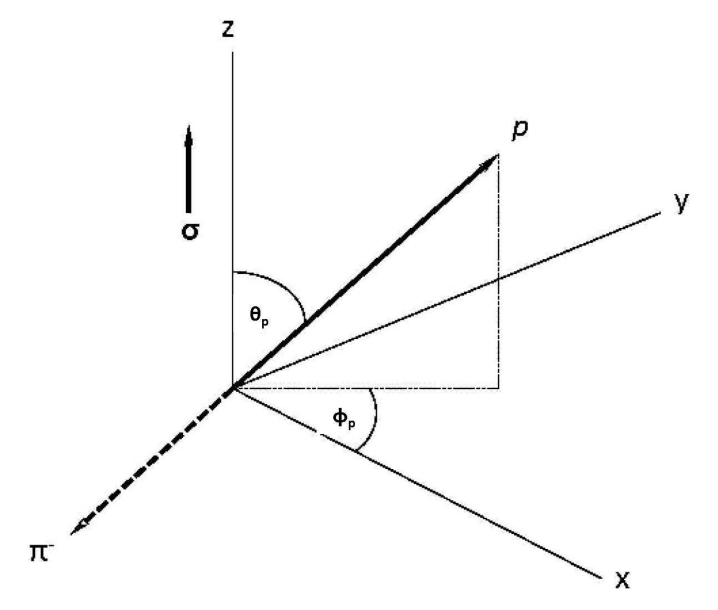

Figure 1.3: $\Lambda$ decay axes definitions [3].

angular momentum must be conserved as well as the third component of the angular momentum in the decay process. Recall that for the $\Lambda$, the total angular momentum is $J=\frac{1}{2}, J_{z}= \pm \frac{1}{2}$. The proton and pion can be produced with a relative orbital angular momentum $l$. The angular distribution $(\theta, \phi)$ of the decay products will depend on their relative orbital angular momentum. Two possible options for $l$ are $l=0$ ( $s$-wave) with the $p$ and $\Lambda$ spins aligned or $l=1$ ( $p$-wave) with the $p$ and $\Lambda$ spins antialigned. If we call the $z$-components of the proton spin $m_{1}$, the $l$ orbital angular momentum $m_{2}$, and the angular momentum wave function $Y_{m_{2}}^{l}$, then for the $s$-wave $m_{1}=+\frac{1}{2}, m_{2}=0$ and $Y_{m_{2}}^{l}=Y_{0}^{0}$ so that:

$$
\psi_{s}=a_{s} Y_{0}^{0} \chi^{+}
$$

where $a_{s}$ is the amplitude and $\chi^{+}$is the proton spin-up wave function $\left(m_{1}=+\frac{1}{2}\right)$. For the $p$-wave case the conservation of the third component of the total angular momentum $\left(m_{1}+m_{2}=J_{z}=+\frac{1}{2}\right)$ can be achieved either with $m_{1}=+\frac{1}{2}$ and $m_{2}=0$ or $m_{1}=-\frac{1}{2}$ and $m_{2}=1$. Using the Clebsch-Gordan coefficients from [7], the $p$-wave function becomes:

$$
\psi_{p}=a_{p}\left[\sqrt{\frac{2}{3}} Y_{1}^{1} \chi^{-}-\sqrt{\frac{1}{3}} Y_{1}^{0} \chi^{+}\right] .
$$


The total wave function is the sum of the $s$ and $p$ waves:

$$
\psi=\psi_{s}+\psi_{p}=\left[a_{s} Y_{0}^{0}-\frac{a_{p}}{\sqrt{3}} Y_{1}^{0}\right] \chi^{+}+\left[a_{p} \sqrt{\frac{2}{3}} Y_{1}^{1}\right] \chi^{-}
$$

$a_{s}$ and $a_{p}$ are complex amplitudes with an arbitrary phase. One can choose the phase of $a_{s}$ in such a way as to make it real. Using the orthogonality of the $\chi^{+}$and $\chi^{-}$states and replacing the $Y_{m}^{l}$ by their expressions (see e.g. [7]), $Y_{0}^{0}=1, Y_{1}^{0} / \sqrt{3}=\cos \theta_{p}^{R F}$, $\sqrt{\frac{2}{3}} Y_{1}^{1}=-\sin \theta_{p}^{R F}$, the probability amplitude of this angular distribution of the protons can be written as:

$$
\begin{aligned}
\psi \psi^{*} & =\left|a_{s}\right|^{2}+\left|a_{p}\right|^{2} \cos ^{2} \theta_{p}^{R F}+\left|a_{p}\right|^{2} \sin ^{2} \theta_{p}^{R F}-a_{s} \cos \theta_{p}^{R F}\left[a_{p}+a_{p} *\right] \\
& =\left|a_{s}\right|^{2}+\left|a_{p}\right|^{2}-2 a_{s} \operatorname{Re} a_{p}^{*} \cos \theta_{p}^{R F} \\
& =\left(\left|a_{s}\right|^{2}+\left|a_{p}\right|^{2}\right)\left(1-\frac{2 a_{s} \operatorname{Re} a_{p}^{*}}{\left|a_{s}\right|^{2}+\left|a_{p}\right|^{2}} * \cos \theta_{p}^{R F}\right) .
\end{aligned}
$$

By making a substitution:

$$
\begin{aligned}
\alpha & =\frac{2 a_{s} \operatorname{Re} a_{p}^{*}}{\left|a_{s}\right|^{2}+\left|a_{p}\right|^{2}} \\
N_{0} & =\left|a_{s}\right|^{2}+\left|a_{p}\right|^{2},
\end{aligned}
$$

the angular distribution of the decay products can be brought to the form:

$$
\frac{d N}{d \cos \theta_{p}^{R F}}=N_{0}\left(1-\alpha \cos \theta_{p}^{R F}\right)
$$

Recall now that the angle $\theta_{p}^{R F}$ in this expression is defined with respect to the $\Lambda$ spin direction, but this direction is arbitrary. In order to measure the angular distribution experimentally, $\theta_{p}^{R F}$ needs to be redefined with respect to the normal to the production plane. If the $\Lambda$ spin and the normal are assumed to point in the same direction then Eq. 1.30 becomes:

$$
\frac{d N}{d \cos \theta_{p}^{R F}}=N_{0}\left(1-\alpha P \cos \theta_{p}^{R F}\right)
$$


In our analysis, the normal to the production plane is defined as a cross product of the $\mathrm{CM}$ momenta of the $\Lambda$ and virtual photon and is assumed to be directed opposite to the $\Lambda$ spin. With this definition of the normal, Eq. 1.31 becomes:

$$
\frac{d N}{d \cos \theta_{p}^{R F}}=N_{0}\left(1+\alpha P \cos \theta_{p}^{R F}\right)
$$

where $P$ is the average polarization of the $\Lambda$. Eq. 1.32 shows that the polarization, $P$, can be extracted from the angular distribution only if $\alpha \neq 0$, which is possible when both $s$ and $p$ waves are present. Parity violation is the result of the interference between the $s$ and $p$ waves, which gives rise to an asymmetry in the angular distribution. Experimentally, $\alpha=0.642 \pm 0.13$ [7].

The polarization can be extracted by two methods:

1) By fitting a first degree polynomial to the proton angular distributions in the $\Lambda$ rest frame. In this case, $\alpha P=\frac{\text { slope }}{\text { intercept }}$.

2) Forming the forward-backward asymmetry with respect to $\cos \theta_{p}^{R F}=0$ : In this case we integrate Eq. 1.32 from -1 to 0 (backward) and from 0 to 1 (forward), respectively, to get the corresponding yields:

$$
\begin{aligned}
& N^{+}=\int_{0}^{1} N_{0}\left(1+\alpha P \cos \theta_{p}^{R F}\right) d \cos \theta_{p}^{R F}=N_{0}+N_{0} \frac{\alpha P}{2} \\
& N^{-}=\int_{-1}^{0} N_{0}\left(1+\alpha P \cos \theta_{p}^{R F}\right) d \cos \theta_{p}^{R F}=N_{0}-N_{0} \frac{\alpha P}{2} .
\end{aligned}
$$

One can define the asymmetry as:

$$
A=\frac{N^{+}-N^{-}}{N^{+}+N^{-}}=\frac{\alpha P}{2} .
$$

The polarization can be expressed in terms of the asymmetry $A$ as:

$$
P=\frac{2 A}{\alpha}=\frac{2}{\alpha} \cdot \frac{N^{+}-N^{-}}{N^{+}+N^{-}} .
$$




\subsection{Theoretical Models}

In this section we continue the discussion started in Section 1.2, regarding the non perturbative nature of QCD in the nucleon resonance region. While at high energies the quark interactions are described by QCD predictions, at nucleon resonance energies different theoretical models, which are approximations to QCD, must be employed as justified in Section 1.2. Three major classes of models that try to describe the strangeness photo- and electroproduction are the traditional hadrodynamic models, coupled-channel and Regge plus Resonance (RPR) approaches.

\section{Hadrodynamic Models}

The hadrodynamic or isobar models are derived from an effective Lagrangian approach. The Lagrangian is constructed from tree-level Born terms, which correspond to $p, K$, and $Y$ exchanges and extended Born terms, corresponding to associated resonances, in the $s, t$, and $u$ reaction channels, shown in Fig. 1.2. Only first order terms are included in the calculations, since both photons and electrons do not interact strongly with the target nucleons, which occurs in pion induced reactions. In other words, only one exchange particle is allowed in the intermediate state. Within the general framework of the hadrodynamic approach, models differ by the choice of resonance diagrams in their calculations. Depending on this choice, very different conclusions can be drawn.

One of the limitations of this type of model is the fact that there is no consistent way of including resonances with spins $\geq 5 / 2$. Another limitation is the large number of model parameters involved in the calculations, which prevents one from drawing any clear conclusions about the existence of any missing resonances.

The predictions of the hadrodynamic model by Mart and Benhold (MB) $[12,13]$ have been compared with SAPHIR [20] and CLAS [22, 24, 27, 32, 33] cross-section and polarization data. The coupling constants in this model were determined from the fits to existing kaon capture $\left(K^{-} p \rightarrow \gamma Y\right)$ and $K Y$ photoproduction data. The CLAS data were not included in the fits. Mart and Benhold (MB) included several 
established $s$-channel resonances in their calculations, listed in the PDG, as well as a D13(1960) missing resonance, in an attempt to explain the broad bump observed in the cross section around $1900 \mathrm{MeV}$. The choice of D13(1960) was motivated by the constituent quark model (CQM) by Capstick and Roberts [11], which predicts contributions from $S 11(1945), P 11(1975), P 13(1950)$ and $D 13(1960)$ resonances with significant couplings to the $K \Lambda$ final state. No $u$-channel hyperonic resonances were included in the model. MB results excluded the first three states, requiring only the missing D13(1960) to explain the data. On the other hand, Saghai in Ref. [16], analyzing the same data, shows that fine tuning the $u$-channel background eliminates the need for including any $s$-channel missing resonances.

The MB calculations of $\Lambda$ recoil polarization are not very sensitive to inclusion of the D13(1960) missing resonance in the model, as shown in Ref. [13]. $\Lambda$ recoil polarization turned out to be a non-suitable candidate for further studying this resonance with the MB model.

\section{Coupled-Channel Models}

Several coupled-channel approaches were developed to simultaneously describe pionand photon induced reactions. The importance of this approach is signified in Ref. [14, 16, 17]. As was shown in Ref. [14], it is necessary to take into account the multistep process $\gamma N \rightarrow \pi N \rightarrow K Y$, where the non-resonant pion-nucleon state is produced as an intermediate state in strangeness production. This effect is known as meson clouding effect. It was shown that it can have up to a $20 \%$ impact on the total cross section of $\gamma N \rightarrow K Y$ direct production [15].

The latest dynamical coupled-channel approaches also take into account the off-shell effects at the vertices, associated with including spin-3/2 resonances. These effects have been integrated over in effective-field models. High-spin resonance contributions become very important at higher $W$, where the most missing resonances are predicted. The off-shell effects can play a significant roll when interpreting the decay properties 
and drawing any clear conclusions about the missing resonances involved in the reaction. In Ref. [14] these effect were taken into account by including $K Y \rightarrow K Y$ as an intermediate state in the production process.

The coupled-channels model by Julia-Diaz [18], after fitting CLAS photoproduction data, claim the contributions from known resonances $S 11$ (1535), P13(1900), D13(1520) into the $\gamma N \rightarrow K Y$ reaction. Three new resonances were also required by this model to describe the experimental data. The most significant contribution is from $D 13(1954)$, and, to a lesser extent, from $S 11(1806)$. They also do not exclude small contributions from P13(1893). The coupled-channels model by Sarantsev [19] demands the presence of $P 11(1840)$ and $D 13(2170)$ in addition to $D 13(1954)$ in order to describe the CLAS and SAPHIR photoproduction data. Shklyar [17], on the other hand, identifies $S 11(1650), P 13(1720)$ and $P 13(1900)$ as the main contributors, and claims that there is no need to include any missing resonances to describe the CLAS and SAPHIR photoproduction data.

The constantly growing high quality database of cross sections and polarization observables for $\pi N \rightarrow K Y$ and $\gamma N \rightarrow K Y$ creates a very favorable environment for

developing and testing coupled-channel models. Electroproduction provides access to interference response functions that are not accessible by any other means. Adding the results of the current analysis to the world database of strangeness production will allow the theorists to also incorporate electroproduction data into their models for simultaneous fits.

\section{Regge plus Resonances}

The last class of models are the RPR models. The RPR approach is also an effectivefield model starting from Feynman diagrams. However, the standard Feynman propagators are replaced by Regge propagators in the amplitude calculations:

$$
\frac{1}{t-m_{X}^{2}} \rightarrow \quad P_{\text {Regge }}^{X}\left[s, \alpha_{X}(t)\right]
$$


The non-resonant background contributions are treated as exchanges of kaonic Regge trajectories in the $t$-channel, with $K(494)$ and $K^{*}(892)$ as dominant trajectories of the form $\alpha_{X}(t)=\alpha_{X, 0}+\alpha_{X}^{\prime}\left(t-m_{X}^{2}\right)$. The corresponding propagators are given by:

$$
P_{R e g g e}^{K^{(*)}}(s, t)=\left(\frac{s}{s_{0}}\right)^{\alpha_{K^{(*)}}(t)} \frac{1}{\sin \left(\pi \alpha_{K^{(*)}}(t)\right)} \frac{\pi \alpha_{K^{(*)}}^{\prime}}{\Gamma\left(1+\alpha_{K^{(*)}}(t)\right)}\left\{\begin{array}{c}
1 \\
e^{-i \pi \alpha_{K^{(*)}}(t)}
\end{array}\right\} .
$$

Here $\alpha_{K}(t)=0.70\left(t-m_{K}^{2}\right)$ and $\alpha_{K^{*}}(t)=1+0.85\left(t-m_{K^{*}}^{2}\right)$ [9] are the Regge trajectories for $K^{+}$and $K^{*}$, respectively, $s$ and $t$ are the standard Mandelstam variables, and $s_{0}$ is a mass scale. Propagators can be used with a constant (1) or rotating $\left(e^{-i \pi \alpha(t)}\right)$ phase, which can be fixed by fitting to high energy photoproduction data. Motivated by existing experimental data, these models then furnish $t$-channel background with established and some missing $s$-channel nucleon resonances in order to explain the structures observed in the cross section and polarization data.

Initially the RPR model was developed for the photoproduction process. The extension to electroproduction was achieved by multiplying the $t$ channel diagrams by the electromagnetic form factor (EMFF) of a monopole form: $F_{K}\left(Q^{2}\right)=\left(1+Q^{2} / \Lambda_{K}^{2}\right)^{-1}$, where $\Lambda_{K}$ is a mass scale. The advantage of this approach is that it greatly reduces the number of model parameters as compared to typical hadrodynamic models. It also eliminates all gauge invariance breaking issues that arise with the inclusion of hadronic form factors.

Ref. [9] includes all established $s$-channel resonances listed in the PDG [7] in their calculations. The missing D13(1900) and P11(1900) resonances were also considered as possible contributors. Comparison of calculations to the $K^{+} \Lambda$ and $K^{+} \Sigma^{0}$ separated cross sections from Ref. [27] and the $K^{+} \Lambda$ transferred polarization electroproduction data from Ref. [32] allowed the authors to exclude P11(1900) as a possible candidate. Only including the $D 13(1900)$ into their calculations lead to reasonable fits of the data. 


\subsection{Previous Experimental Data}

Strangeness production experiments from various nucleon targets have been carried out since the 1970s, but high quality data became available only recently. Differential cross sections and induced hyperon recoil polarization data for $K Y$ photoproduction have been published by the SAPHIR [20], LEPS [21], GRAAL [23], and CLAS [22, 24, 25] collaborations. The published $K Y$ photoproduction data cover the full range of $\cos \theta_{K}^{C M}$ and $W$ from 1.6 to $2.3 \mathrm{GeV}$. Recent photoproduction data by McCracken [26] extended the existing $W$ range by $500 \mathrm{MeV}$ and largely improved the precision of the cross section and hyperon induced polarization data for the $K^{+} \Lambda$ final state.

High statistics data for $K Y$ electroproduction are relatively scarce as compared to photoproduction. Recent data, covering the full kaon center-of-mass angular range, were published by the CLAS collaboration. The separated structure functions $\sigma_{U}, \sigma_{T}, \sigma_{L}$, $\sigma_{T T}$, and $\sigma_{L T}$ for the $K^{+} \Lambda$ and $K^{+} \Sigma^{0}$ final states were published by Ambrozewicz [27]. These high statistics data cover the $Q^{2}$ range from 0.5 to $2.8 \mathrm{GeV}^{2}$ and the $W$ range from threshold to $2.4 \mathrm{GeV}$. In a recent publication from Hall A of Jefferson Lab by Coman [28], the longitudinal, $\sigma_{L}$, and transverse, $\sigma_{T}$, cross sections were separated by the Rosenbluth technique at fixed $W$ and $t$. These results cover the kinematic range for $Q^{2}$ from 1.90 to $2.35 \mathrm{GeV}^{2}$ and $W$ range from 1.80 to $2.14 \mathrm{GeV}$. The first measurement of the polarized structure function $\sigma_{L T}$ in the resonance region by Nasseripour [29] covers the $W$ range from the threshold $(\sim 1.6 \mathrm{GeV})$ up to $2.05 \mathrm{GeV}$ and the $Q^{2}$ range from 0.65 to $1.00 \mathrm{GeV}^{2}$, while spanning the full range of $\cos \theta_{K}^{C M}$. Separation of the longitudinal and transverse cross sections in the $p\left(e, e^{\prime} K^{+}\right) \Lambda, \Sigma^{0}$ reactions was published by Mohring [30, 31]. These Jefferson Lab Hall $\mathrm{C}$ data cover a $Q^{2}$ range from 0.5 to 2 $\mathrm{GeV}^{2}$ at an invariant mass $W=1.84 \mathrm{GeV}$.

Recent beam-recoil transferred polarization data for the exclusive $p\left(\vec{e}, e^{\prime} K^{+}\right) \vec{\Lambda}$ reaction by Carman $[32,33]$ have wide kinematic coverage spanning $Q^{2}$ range from 0.7 to $5.4 \mathrm{GeV}^{2}$ and $W$ range from 1.6 to $2.6 \mathrm{GeV}$. These data [33] extended the existing CLAS data for transferred polarization for $K^{+} \Lambda$ and presented first ever measurements 
for the $K^{+} \Sigma^{0}$ final state.

Previous $\Lambda$ induced polarization data also exist for the exclusive $p\left(e, e^{\prime} K^{+}\right) \Lambda$ reaction. The results of this measurement, performed by S. McAleer [34], have never been published. Because of the lack of statistics, several datasets with different beam energies had to be combined, potentially obscuring the underlying physics. These data span the $Q^{2}$ range from 0.5 to $2.8 \mathrm{GeV}^{2}$ and nearly the entire range of $\cos \theta_{K}^{C M}$. When mapping out the kinematic dependences of the induced polarization, the results had to be integrated over the other kinematic variables in order to reduce the statistical uncertainties. The results were dominated by kaon forward angle data, where the statistics were the largest, preventing any $s$-channel resonance contributions to be observed. 


\section{Experimental Setup}

\subsection{Facility Overview}

This experiment was carried out at the Thomas Jefferson National Accelerator Facility, Newport News, VA. Data were taken during the E1F run period in 2003 using the CEBAF Large Acceptance Spectrometer (CLAS) located in experimental Hall B. A schematic diagram of the facility is shown in Fig. 2.1. The electron bunches are produced in the injector and are sent into the accelerator linac. After each pass through the accelerator, the electrons gain about $1 \mathrm{GeV}$. The maximum beam energy achieved is about $6 \mathrm{GeV}$ with $\Delta E / E \leq 10^{-4}$ energy resolution and a beam spot size of $\sigma<$ $250 \mu \mathrm{m}$. The continuous electron beam from the accelerator can be split between three end stations called Halls A, B, and C. Every third bunch can be delivered to an experimental hall, allowing simultaneous experiments to run in all three halls. Because of the $1497 \mathrm{MHz}$ RF structure of the linac cavities, the electron beam bunches are separated by $2 \mathrm{~ns}$ intervals in each experimental hall. The comprehensive physics program of the

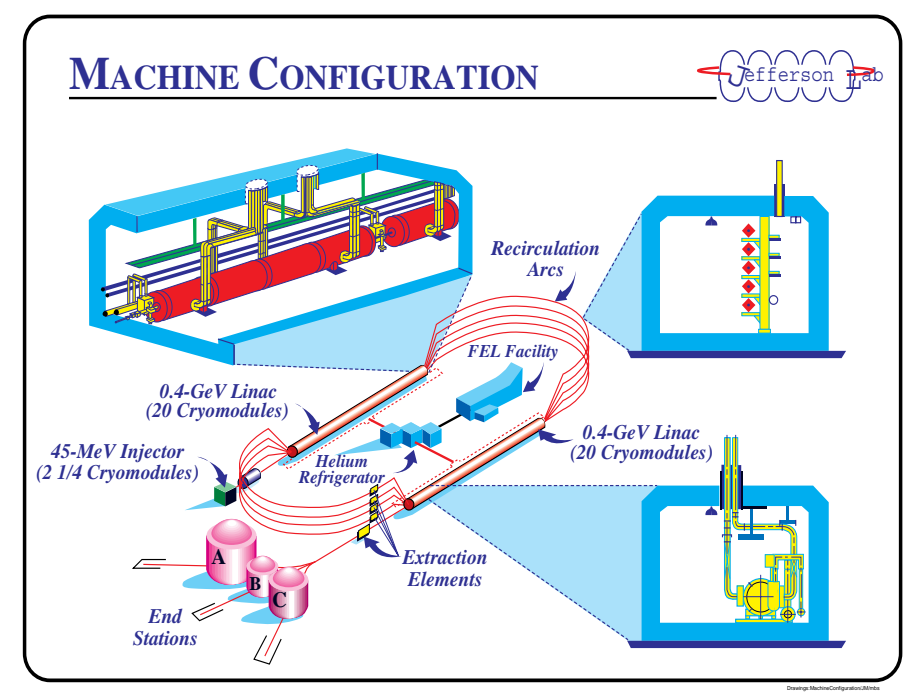

Figure 2.1: Schematic view of the Jefferson Lab accelerator [36]. 
facility is devoted to investigations of the electromagnetic structures of nucleons and mesons with high energy electron and photon beams. The searches for missing baryon resonances and investigations of the spin structure of the nucleons in single and double polarization experiments are also carried out. The Jefferson Laboratory also has a large hypernuclear program. These experiments probe the interaction of lambda hyperons with ordinary nuclear matter. One of the goals is to test unified baryon-baryon interaction models.

\subsection{CLAS Detector}

The Hall B physics program is mainly based on the CEBAF Large Acceptance Spectrometer (CLAS) [35]. The CLAS detector is designed in such a way as to allow operation with both electron and photon beams while providing acceptance coverage of approximately $50 \%$ of $4 \pi$ in solid angle.

The large acceptance of the CLAS is crucial for the investigations of the multiparticle final states that result from the decay of the produced excited baryons and mesons. The volume of the detector is divided into six identical sectors by toroidal magnet coils. Each of the CLAS sectors is equipped with an identical set of detectors: three layers of drift chambers (DC) for charged particle tracking and momentum reconstruction, Cherenkov counters (CC) for electron identification and triggering, scintillation counters (SC) for time of flight measurements and charged particle identification, and electromagnetic calorimeters (EC) for electron identification and triggering. Fig. 2.2 illustrates the schematic view of the CLAS detector subsystems. Each of these components will be discussed in detail in the next sections of Chapter 2 .

The geometrical shape of the CLAS detector allows a large kinematic acceptance in $Q^{2}$ and $W$ as well as hadron scattering angles (from $8^{\circ}$ to $142^{\circ}$ ) and electron scattering angles (from $8^{\circ}$ to $45^{\circ}$ ). In this experiment, a $5.499 \mathrm{GeV}$ polarized electron beam was incident upon an unpolarized liquid-hydrogen target. The scattered electrons and some

of the reaction products are detected by the CLAS spectrometer. This data set covers a 


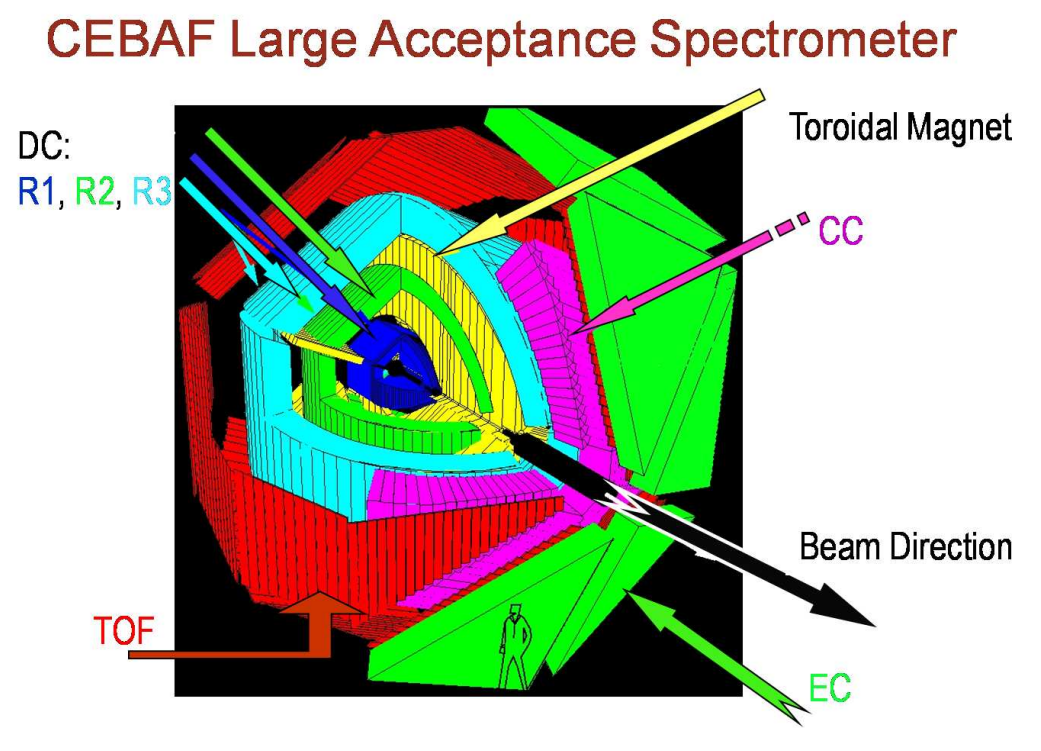

Figure 2.2: Schematic view of the CLAS detector showing all subsystems of the spectrometer.

$Q^{2}$ range from 0.8 to $3.5 \mathrm{GeV}^{2}$ and a $W$ range from threshold $(1.6 \mathrm{GeV})$ to $3.0 \mathrm{GeV}$. Standard spherical coordinates are used in the description of the experimental setup, where the $z$-axis is directed along the beam direction, $\theta$ is the polar angle, and $\phi$ is the azimuthal angle.

The CLAS detector can operate at luminosities up to $10^{34} \mathrm{~cm}^{-2} \mathrm{~s}^{-1}$. One of the major limiting factors for luminosity is the drift chamber accidental hit occupancy resulting from low energy photons and Møller scattering in the target. The flux of low energy particles reaching the drift chambers greatly reduces the tracking efficiency for accidental hit occupancies above 5\% [39].

\section{Main Torus}

The main magnetic field of the spectrometer is provided by six superconducting coils measuring $5 \mathrm{~m}$ in length and spanning $5 \mathrm{~m}$ in diameter [35]. The generated magnetic field is toroidal with its main component in the azimuthal direction. The arrangement of the coils around the beamline is shown in Fig. 2.3. The magnetic field is calculated directly from the current in the coils. The coils are capable of generating magnetic 
fields up to $2.5 \mathrm{~T}$ at forward angles and $0.6 \mathrm{~T}$ at $90^{\circ}$ scattering angles at a maximum torus current of $3860 \mathrm{~A}$. The choice of the toroidal magnetic field is justified by the requirement of keeping the target region free from magnetic fields to make the polarized target experiments possible. The coils and DC readout electronics limit the azimuthal acceptance of CLAS to $80 \%$. During the E1F run period the main torus current was set at $2250 \mathrm{~A}$. The polarity of the magnetic field was set so that negatively charged particles were bent towards the beamline. Since the main component of the magnetic field is azimuthal, the tracks are bent only in the polar direction, while the azimuthal $\phi$ angles of the tracks remain unchanged.

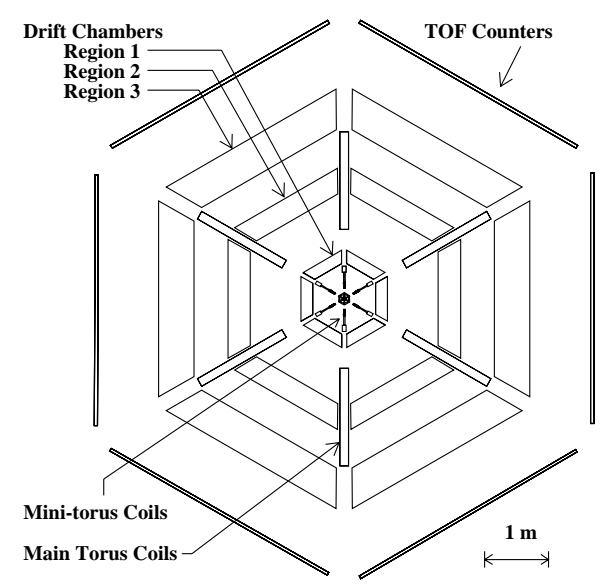

Figure 2.3: Schematic diagram of the CLAS detector. The relative positioning of the main and mini-torus coils are shown with respect to the target position.

\section{Mini-Torus}

The inner layers of the drift chambers are located around the target, inside of the main torus field. In electron scattering experiments, low momentum electrons produced in the target as a result of Møller scattering can reach the inner layers of the drift chambers which will increase the rates and reduce the live time of the chambers. To improve the drift chamber performance, small magnet coils (mini-torus) are placed around the target as shown in Fig. 2.3. The small magnetic field generated by the mini-torus sweeps 
away low momentum electrons directing them into the forward direction and out of the fiducial volume of CLAS, thus preventing them from reaching the drift chambers. The mini-torus current was set at 5995 A during E1F run period.

\section{Drift Chambers (DC)}

The primary goal of the CLAS drift chambers is to provide the charged particle track reconstruction and momentum measurements for particles with energies above $200 \mathrm{MeV}$. The DCs cover the full scattering range from $8^{\circ}$ to $142^{\circ}$ with the azimuthal acceptance limited to $80 \%$. A total of 18 drift chambers are located at three different radial distances from the target in each sector. These positions are referred to as Regions, with Region 1 being the closest to the beamline (Fig. 2.3).

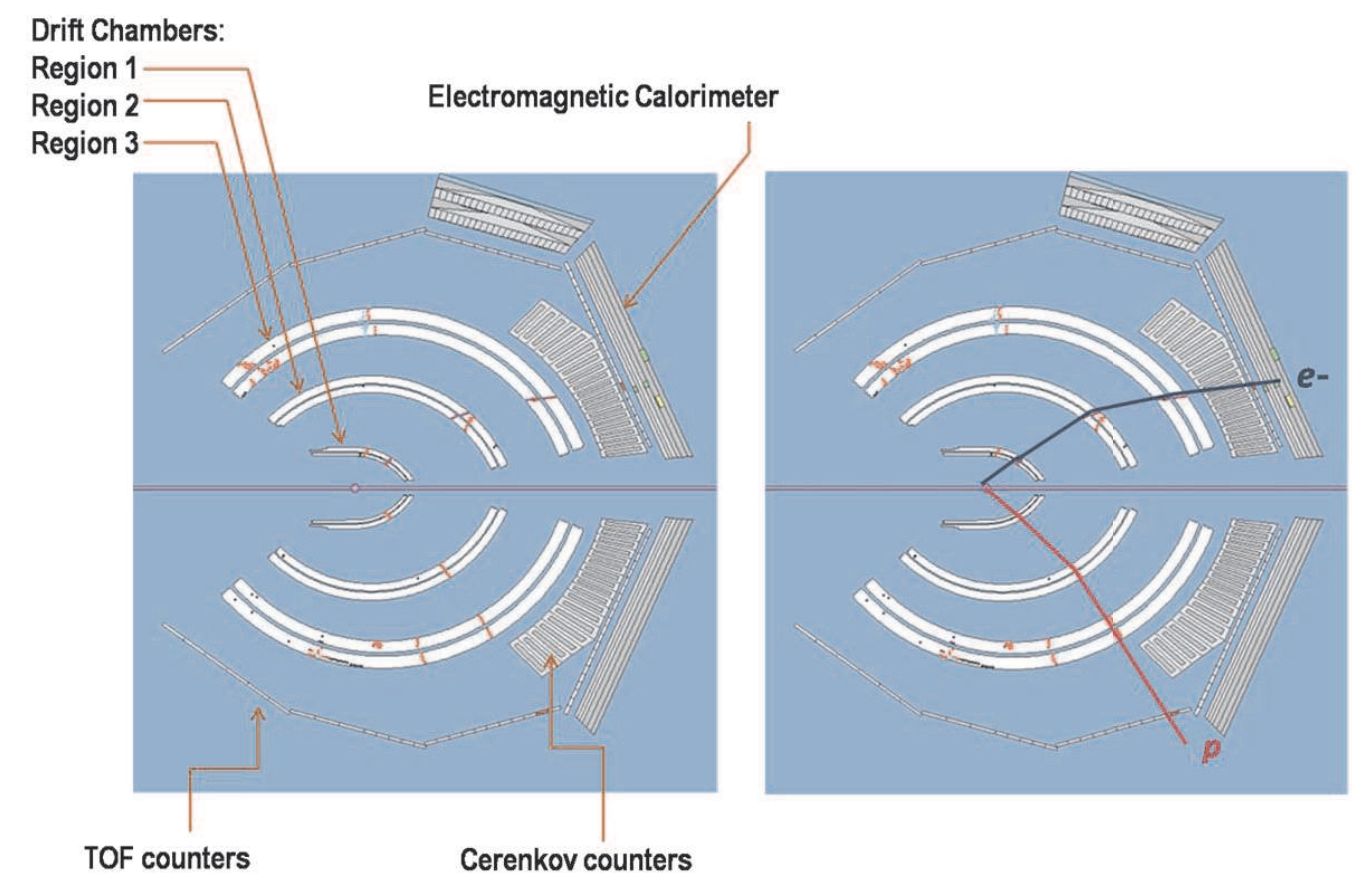

Figure 2.4: Typical CLAS event reconstruction. Negatively charged particles are bent towards the beamline. Track segments are combined in different superlayers of all regions to form the charged particle trajectories that traverse the volume of the CLAS spectrometer.

Regions R1 and R3 are positioned out of the main torus field while R2 is placed between the coils within the field. It is actually mounted on the main torus cryostats. 
The magnetic field in this area is the largest, so the particle trajectories are bent in this region. Each region is subdivided into two superlayers with six layers of sense wires in each, except for R1, where, due to the lack of space, only four layers of sense wires are present. All sense wires are surrounded by six field wires forming hexagonal cells. The wires in one superlayer are arranged parallel to the magnetic field while the wires of the second superlayer are tilted by $6^{\circ}$ with respect to the first. The tiling is done in order to obtain the azimuthal angle information of the particle. There are a total of 35,148 individual sense wires in the drift chamber system. All drift chambers are filled with a $90 \%$ argon and $10 \% \mathrm{CO}_{2}$ gas mixture. This mixture was chosen for safety considerations as well as to improve the operation and lifetime of the tracking system. The tracking resolution is about 310,315 , and $380 \mu \mathrm{m}$ for $\mathrm{R} 1, \mathrm{R} 2$, and $\mathrm{R} 3$, respectively.

The DC track reconstruction is done in two steps. The first step is the hit-based tracking, during which the algorithm identifies the hits and uses the hit wire positions to form the track segment in each superlayer then combines the identified track segments from different regions. Typical event reconstruction is illustrated in Fig. 2.4. The polarity of the magnetic field for this experiment bends the negatively-charged particles towards the beamline. After hit-based tracks are formed, the momentum of the particles can be determined within 3-5\% accuracy because of the small size of the DC cells. The second step is time-based tracking. In this step the time of flight as determined by SC is used to correct the drift times. The corrected drift times are then converted into drift distances by using a look up table created by simulations. These new track segments from different superlayers of all three regions are again combined together to form the new time based track. Overall efficiency of the tracking system exceeds $95 \%$ for up to $4 \%$ hit occupancy, with a momentum resolution of $\delta p / p \leq 0.5 \%$ and angular resolution $\delta \theta, \delta \phi \leq 2 \mathrm{mrad}$. In order to do particle identification, the tracks are matched in software with timing and energy-loss information from outer detector components. Additional information about the CLAS tracking system can be found in Ref. [39]. 


\section{Cherenkov Counters (CC)}

The CLAS Cherenkov counters are intended for electron identification and are also used in trigger formation. They cover a scattering-angle range from $8^{\circ}$ to $45^{\circ}$ in all six sectors. Each module of the Cherenkov counters contains elliptical, hyperbolic, and cylindrical mirrors to transport the light to photomultiplier tubes (PMTs). The CC optical system is schematically shown in Fig. 2.5. They are designed to focus light only

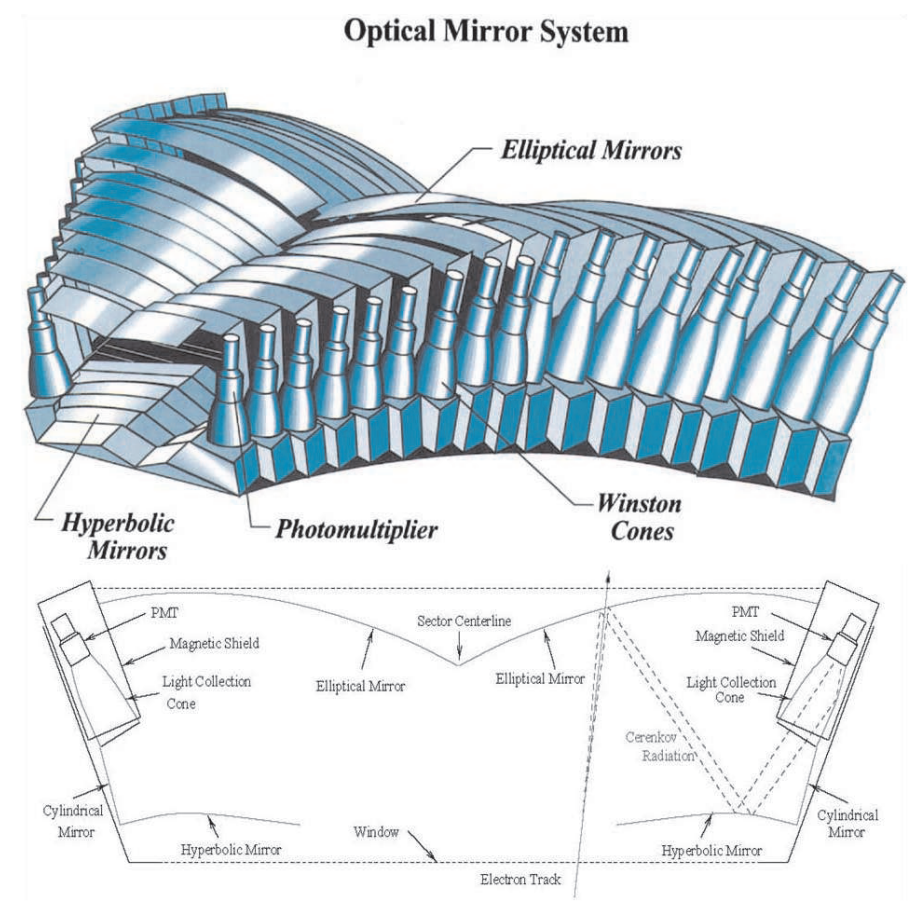

Figure 2.5: CC Optical mirror system.

in the $\phi$ direction thus preserving the polar angle information of the electron. There are a total of $18 \theta$-strips, which are also divided into 2 azimuthal segments with the midplane of each sector acting as a symmetry plane between them (see Fig. 2.5). So, each $\theta$ strip is covered by 12 identical CC modules in the $\phi$ direction. All PMTs are placed in the regions partially covered by the torus coils in order to avoid further limitation of the acceptance. The volume of the detector is filled with $\mathrm{C}_{4} \mathrm{~F}_{10}$ radiator gas with a refractive index $n=1.00153$. 
The Cherenkov radiation threshold expressed in terms of the particle energy and mass is:

$$
E / m \geq \frac{1}{\sqrt{1-\frac{1}{n^{2}}}},
$$

where $n$ is the refractive index of the medium. For electrons, the Cherenkov radiation threshold in this medium is $9.24 \mathrm{MeV}$, while for pions, it is about $2.56 \mathrm{GeV}$. The $\mathrm{CC}$ can effectively separate electrons from pions below energies of $2.56 \mathrm{GeV}$. Above this threshold, the signals from EC must be used for electron/pion identification. The CC in coincidence with EC, is used in the Level 1 trigger formation by telling the master readout that an electron was likely.

The efficiency of the Cherenkov detectors is about $99 \%$ inside the fiducial volume. The studies of the CC efficiency was performed using ep elastic scattering using the coplanarity of the scattered particles. The reconstructed electron scattering angles $\left(\theta_{e}, \phi_{e}\right)$ can be used to uniquely project the electron entry points on the $\mathrm{CC}$ surface. For further information about the CC, please refer to Ref. [40].

\section{Forward Electromagnetic Calorimeter (EC)}

The forward electromagnetic calorimeter is intended for electron identification and triggering above $0.5 \mathrm{GeV}$. It is also capable of detecting photons above $0.2 \mathrm{GeV}$ for $\pi^{0}$ and $\eta$ reconstruction. For neutron detection and discrimination from photons, the EC information is used together with time of flight measurements.

The forward electromagnetic calorimeter covers the polar angles from $8^{\circ}$ to $45^{\circ}$ in all six sectors. EC modules have the shape of an equilateral triangle with a base area of 8 $\mathrm{m}^{2}$. Each module consists of 39 layers of a lead-scintillator sandwich. Every successive layer has a little larger area than the previous one in order to fully contain the electromagnetic shower. Each layer consists of $10 \mathrm{~mm}$ thick BC412 scintillator followed by a $2.2 \mathrm{~mm}$ thick lead sheet. All EC scintillator layers consist of 36 slices parallel to one side of the triangle. Each layer is rotated by $120^{\circ}$ with respect to the previous layer, thus creating three configurations called U, V and W planes as shown in Fig. 2.6. The 
13 layers of each plane are further grouped in 5 (inner) and 8 (outer) layer stacks intended for obtaining the information about the longitudinal progress of the showers and improving hadron identification. The algorithm of the EC hit reconstruction first selects

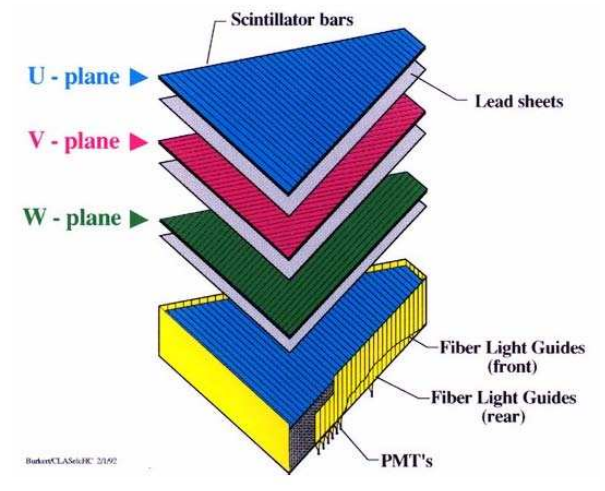

Figure 2.6: CLAS electromagnetic calorimeter modules. U, V and W layers are shown.

the strips and groups them together in each of the U, V, and W planes. Signals from all three planes are required for the reconstruction. The second step is to find the intersection points. Each of the intersection points corresponds to a hit as shown in Fig. 2.7. If there is more than one hit in a single plane, then the weighted energy of the group can be assigned to all hits. In CLAS, electrons and pions are separated by Cherenkov counters for up to $2.5 \mathrm{GeV}$. Above $2.5 \mathrm{GeV}$ pions exceed the Cherenkov radiation threshold making $e^{-} / \pi^{-}$separation impossible by the CC. Instead, the EC is used for separating the electrons from the fast moving pions. Electrons and pions deposit their energies into the calorimeter by two different mechanisms. The electrons deposit their energy by producing $e^{+} e^{-}$cascades. The energy deposition mechanism by shower creation is momentum dependent. Unlike electrons, the pions deposit approximately a fixed amount of energy (about $40 \mathrm{MeV}$ ) by ionization, practically independent of their momentum. The appropriate cut on the energy deposited in the EC can separate the fast moving pions from electrons as described in Section 3.1. The calorimeter can measure only a fraction of the incident particle's energy, since the charged particles, while propagating through the calorimeter, also interact with the lead atoms. This fraction is called a sampling fraction (S.F.) and it is an intrinsic property of the calorimeter. For electron 


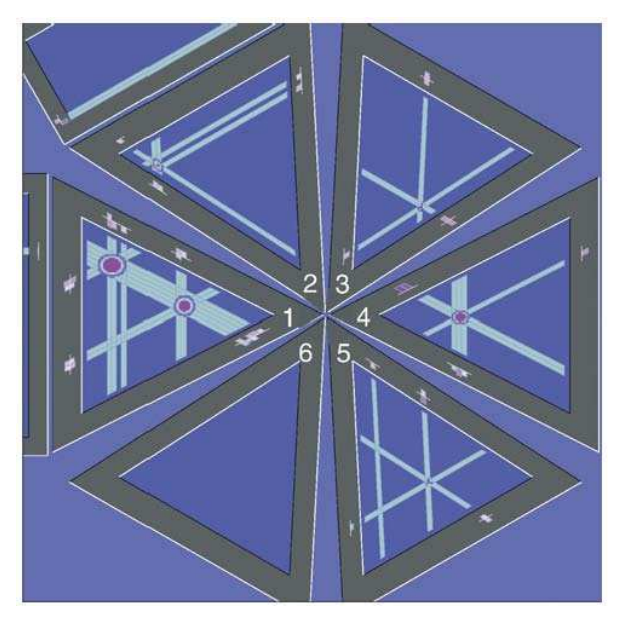

Figure 2.7: CLAS event reconstruction by EC.

energies above $3.0 \mathrm{GeV}$, the S.F. of the calorimeter is about 0.3. Energy resolution for electrons is expressed by:

$$
\frac{\sigma}{E} \leq \frac{0.1}{\sqrt{E(\mathrm{GeV})}}
$$

The position resolution provided by the $\mathrm{EC}$ is $\sim 2 \mathrm{~cm}$ and the timing resolution is $\sim 200$ ps for electrons and $\sim 600$ ps for neutral particles, which is comparable with SC timing resolution. For additional information, please refer to Ref. [38].

\section{Time of Flight System (TOF)}

The CLAS TOF system consists of scintillation counters (SC) for time of flight measurements, the event start time determination, and triggering. The TOF system, along with the DC, is used for charged particle identification, since the momentum of the particle alone is not enough to determine its mass. Detailed discussion of charged particle identification is given in Chapter 3.

The TOF paddles are long plastic scintillators (Bicron BC-408) with one PMT attached at each end. The positioning of the TOF paddles relative to other subsystems of CLAS can best be seen from Fig. 2.4. They are located outside of the tracking system and Cherenkov counters, but before the electromagnetic calorimeters. There are a total of 57 paddles in each sector mounted in four panels covering the scattering-angle range 


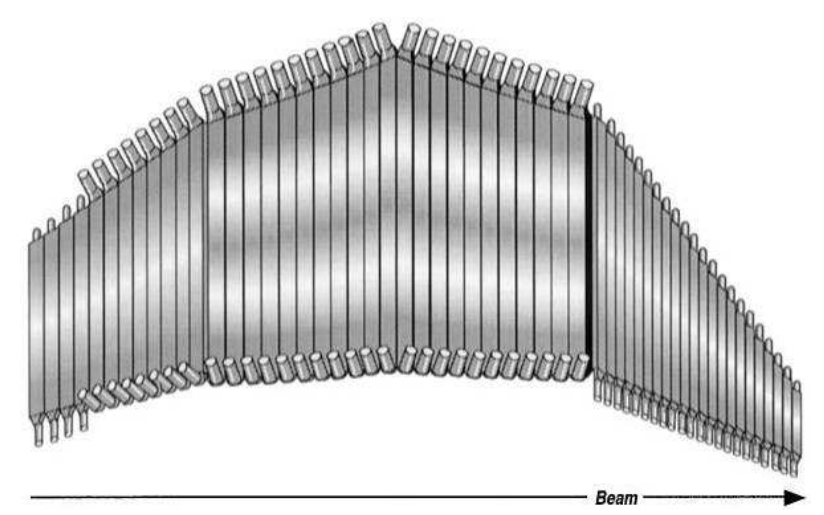

Figure 2.8: TOF scintillator paddles for one sector.

from $8^{\circ}$ to $142^{\circ}$ (Fig. 2.8). The geometrical sizes of the paddles are chosen to optimize the timing resolution at the $\sim 100 \mathrm{ps}$ level and allow the TOF system to operate at rates of about $100 \mathrm{kHz}$. The scintillator paddle lengths range from $32 \mathrm{~cm}$ to $445 \mathrm{~cm}$, with a thickness of $5.08 \mathrm{~cm}$. All scintillators on panel 1 (covering the scattering angles $<45^{\circ}$ ) and the last four on panel 4 measure $15 \mathrm{~cm}$ in width. The rest of the scintillators are $22 \mathrm{~cm}$ wide. Each paddle covers about $2^{\circ}$ in polar angle. The fine segmentation allows the TOF to be used in Level 1 trigger formation. The last 18 scintillator counters are paired together, forming a total of 48 logical counters in each sector. The achieved time resolution is about 120 ps or better at forward angles and about $250 \mathrm{ps}$ at angles greater than $90^{\circ}$. For more information about the SC, please refer to Ref. [41].

\subsection{Cryogenic Target}

The experimental target used during E1F run period is shown in Fig. 2.9. The target cell is $50 \mathrm{~mm}$ long and filled with liquid hydrogen. It was positioned $\sim 25 \mathrm{~cm}$ upstream from the center of the CLAS. A 0.001" Kapton film is cut to fit the cell wall. 5 layers of super-insulation, each with one layer of Cerex $\left(1.0 \mathrm{mg} / \mathrm{cm}^{2} / \mathrm{Layer} / \mathrm{Ply}\right)$, are surrounding the cell wall. The target cell has a $12 \mathrm{~mm}$ inner diameter at the base and a $7 \mathrm{~mm}$ diameter at the downstream end. The enlarged base of the cell was intended to allow gas bubbles to escape easily. The target cell entrance and exit windows are made of $15 \mu \mathrm{m} \mathrm{Al}$ 


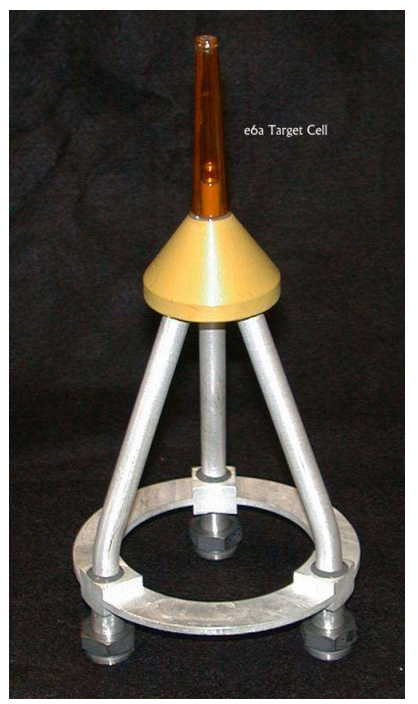

Figure 2.9: E1F cryogenic target.

and are about $4 \mathrm{~mm}$ in diameter. Target parameters, maintained during the run period are summarized in Table 2.1. Although multiple empty target runs were performed to estimate the contributions from the target walls, no separate subtraction of target wall contributions were performed in this analysis. Instead, target wall contributions are removed in the background subtraction process, described in Section 4.4.

\begin{tabular}{|r|r|}
\hline Length & $50 \mathrm{~mm}$ \\
\hline Temperature & $\sim 20.5 \mathrm{~K}$ \\
\hline Density & $\sim 0.0704 \mathrm{~g} / \mathrm{cm}^{3}$ \\
\hline Pressure & $\sim 1230 \mathrm{mb}$ \\
\hline Z-location & $-25 \mathrm{~cm}$ \\
\hline
\end{tabular}

Table 2.1: Hydrogen cryotarget parameters during the E1F run period.

\subsection{Data Acquisition and Trigger System}

The event readout from CLAS is initiated by the two-level trigger. The Level 1 trigger processes the PMT signals from different CLAS components, forming coincidences between EC, CC, and TOF scintillators and sending them to the trigger supervisor (TS). When a Level 1 trigger occurs, the TS generates the gates for PMT time-to-digit con- 
verters (TDCs) and signals the analog-to-digit converter (ADC) to integrate the PMT signals within the gates. The TS also generates the common stop for the drift chamber TDCs.

The Level 1 trigger can be used by itself or together with the Level 2 trigger to initiate event readout. The Level 2 trigger checks for possible track segments in five out of six superlayers of the drift chambers. If track segments are present in at least three superlayers, then a comparison is made with previously generated templates to confirm or reject a possible track. The Level 2 trigger can also be configured to check for coincidences between DC, EC, CC, and TOF signals by extrapolating the tracks. If there are no track candidates available in CLAS, then the Level 2 trigger fails, sending a fast clear signal to the TS. Upon receiving the fast clear signal, the TS resets all electronics and is ready to accept new events.

In case possible track candidates are found, the TS receives a Level 2 trigger confirmation, and the information is collected from all CLAS subsystems, digitized and sent to a process called the Event Builder (EB). Until this digitization process is finished, no new events can be accepted. The EB then groups the information from CLAS subsystems into separate banks, forming a complete event, labels them by a unique event number within the current run, and sends them to the Event Recorder (ER). Finally, the ER writes the information to a tape silo for permanent storage. Complete information about the run conditions like beam energy and current, magnetic field settings, applied high voltages, triggers, target etc. are permanently stored in the run database and can be accessed at any time during the offline data analysis. During the data acquisition, some portions of the data were constantly analyzed by the online monitoring programs in order to check for the detector performance and the quality of the data. 


\section{CHAPTER 3}

\section{Particle Identification}

Careful particle identification is required to select the final state of interest in addition to the initial track reconstruction. The complete particle identification procedures and the cuts employed to select the $p\left(e, e^{\prime} K^{+}\right) \Lambda$ final state are described in the following sections of this chapter.

\subsection{Electrons}

The trigger for this experiment was the coincidence between an EC and a $\mathrm{CC}$ in the same sector. This trigger configuration ensures that all events have an electron candidate. Electron candidates are also required to have a valid track in the DC corresponding to a negatively charged particle and a hit in the time of flight SC system that coincides in time with the hit in the EC. The events, for which these conditions are not satisfied, are rejected in the offline analysis during reconstruction. These are the minimum requirements for electron candidates. They still can include rescattered electrons, electrons that hit non-fiducial regions of the detector and background pions that accidentally created a signal in CC and passed all other electron requirements. During the course of the analysis we performed detailed studies of the standard set of cuts previously used for CLAS analysis. This standard set of cuts include:

- Cut on the number of photoelectrons in the $\mathrm{CC}$

- EC energy cuts

- EC fiducial cuts

- Geometrical fiducial cuts

- $z$-Vertex position cut 


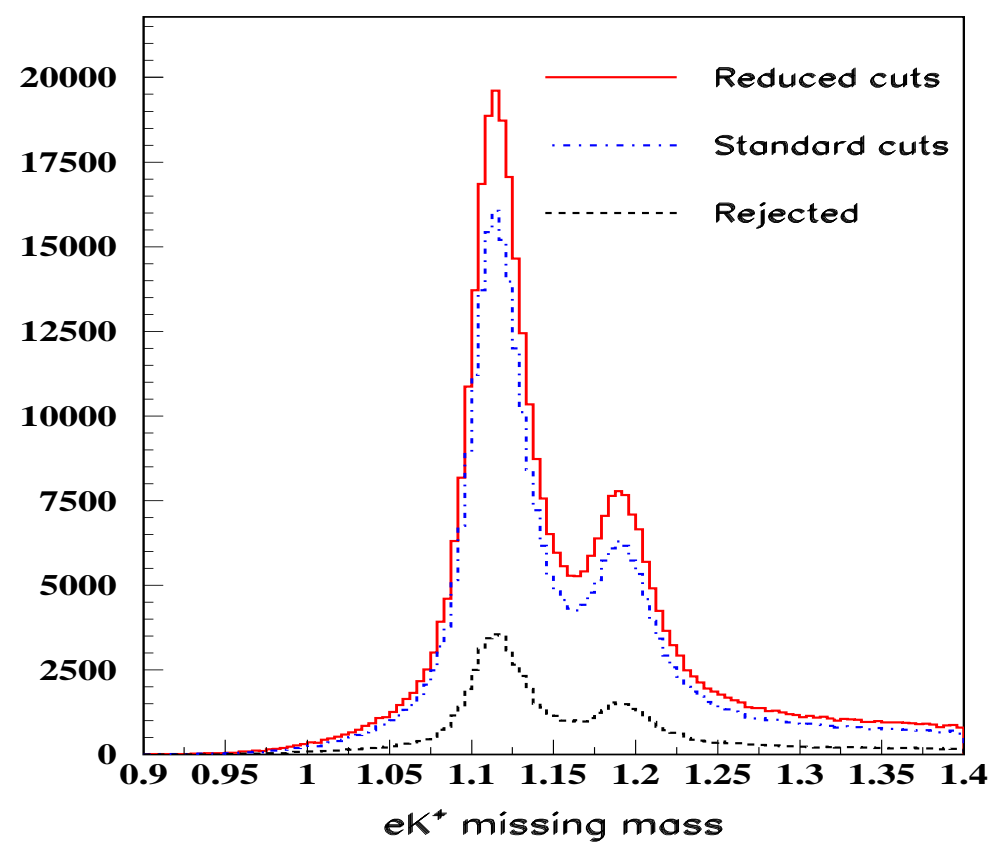

Figure 3.1: Reconstructed $\Lambda$ mass with reduced (red curve) and standard (blue dot-dashed curve) electron cuts. The black dashed curve corresponds to events rejected by the removed cuts.

It became clear that most of these cuts instead of improving signal to background ratio, are removing mostly good events. The exclusivity of the reaction of interest, namely requiring detection of scattered a electron, kaon, and proton, along with the cuts on the hadron side, make the electron cuts overly stringent. Removing some of the cuts listed above recovered about $1 / 3$ of the data sample. Fig. 3.1 shows the $\Lambda$ missing mass spectrum with standard and reduced electron cuts in place. It clearly shows that most of the removed events are good events. The reconstructed $\Lambda$ mass for rejected electrons by any of the above listed cuts is illustrated in Fig. 3.2 for backward kaon CM angles. The statistics of the process is already limited at backward angles as it is, so preserving as many good events as possible at these kinematic bins is especially crucial. These plots show that removing some of these cuts are justified. Of course, these cuts are analysis specific. For polarization measurement we can reduce the number of $e^{-}$cuts because we do not care about $e^{-}$acceptance. Only $p$ acceptance plays a significant role for this 

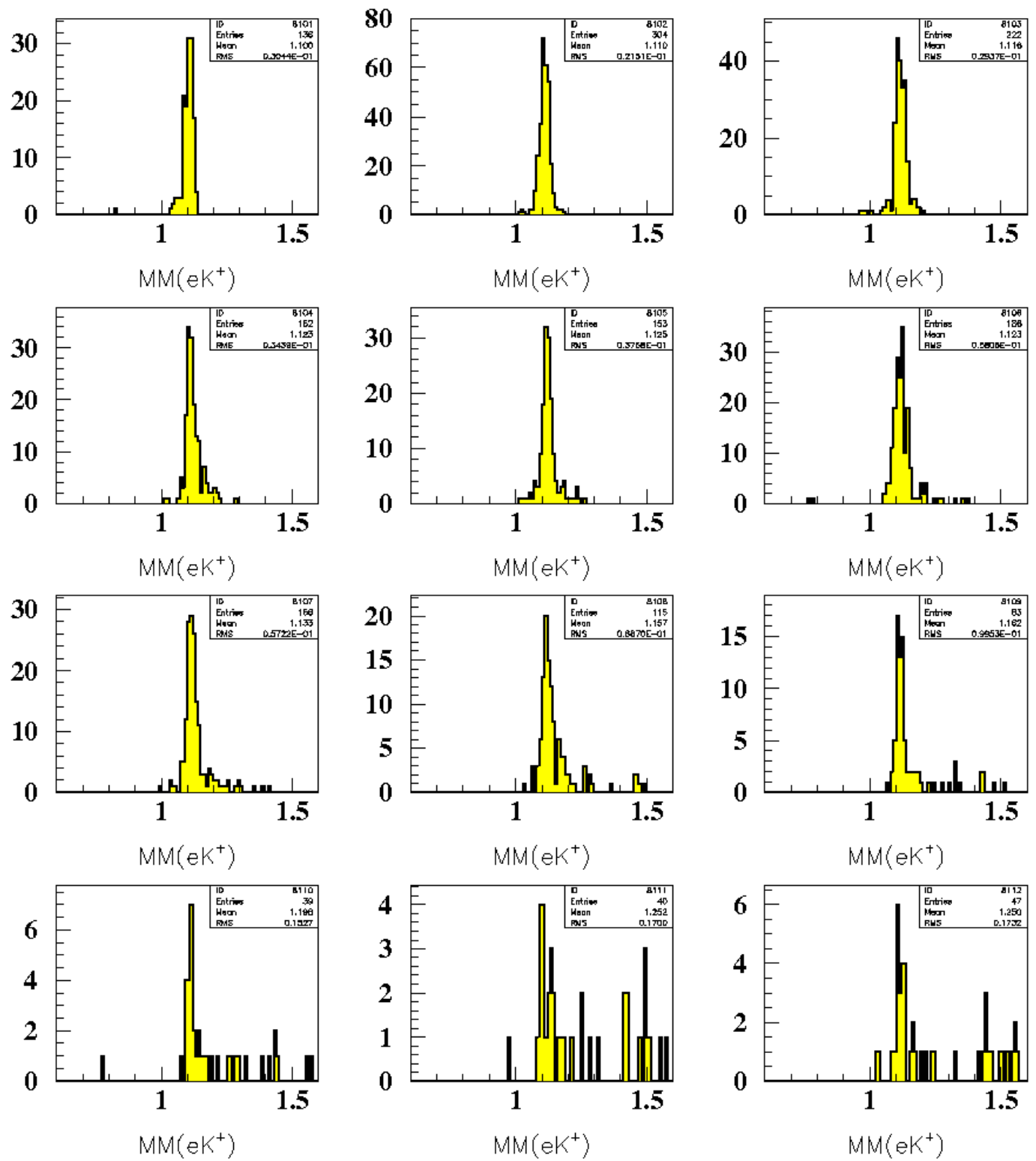

Figure 3.2: Reconstructed $\Lambda$ mass for events rejected when all electron cuts are applied, at backward kaon center of mass angles $-1.0<\cos \theta_{K}^{C M}<-0.5$ for different $W$ bins starting from $1.6 \mathrm{GeV}$ to $2.2 \mathrm{GeV}$ with $50 \mathrm{MeV}$ bins. 
measurement. The $e^{-}$and $K^{+}$acceptances effectively cancel out in asymmetry method used for polarization calculations. For a cross-section measurement, on the other hand, the $e^{-}$acceptance is crucial. In the final analysis, to clean up the electron samples, the following cuts were applied.

- Electron $z$-vertex cuts: Intended for selecting the events that have an interaction vertex in the target region. This cut ensures the proper track reconstruction, which will affect momentum and time of flight measurements, thus hadron identification.

- Electromagnetic calorimeter fiducial cuts: These cuts are kept in order to ensure that the electromagnetic shower is fully contained within the EC volume to avoid "leaks" of deposited energy. It is crucial for particle identification and proper energy measurements.

- Electron geometrical fiducial cuts: Geometrical fiducial cuts are kept in order to select the CLAS fiducial regions where the acceptance is well understood. It is needed to avoid large acceptance corrections which will increase the systematic uncertainties of the results.

\section{Photoelectron Cut}

The cut on the number of photoelectrons in $\mathrm{CC}$ is intended as a signal threshold to eliminate the electronic noise and reduce the pion contributions above $2.5 \mathrm{GeV}$ momenta. The usual cut is: $N_{p h e}>25$ shown in Fig. 3.3. The CC photoelectron cut is one of the cuts removed in the present analysis as discussed in Section 3.1.

\section{EC Energy Cuts}

\section{Deposited Energy Cut in EC Inner Layers}

The EC deposited energy cut employs the fact that the electrons and protons deposit their energy into the calorimeter by different mechanisms. The electrons deposit their 


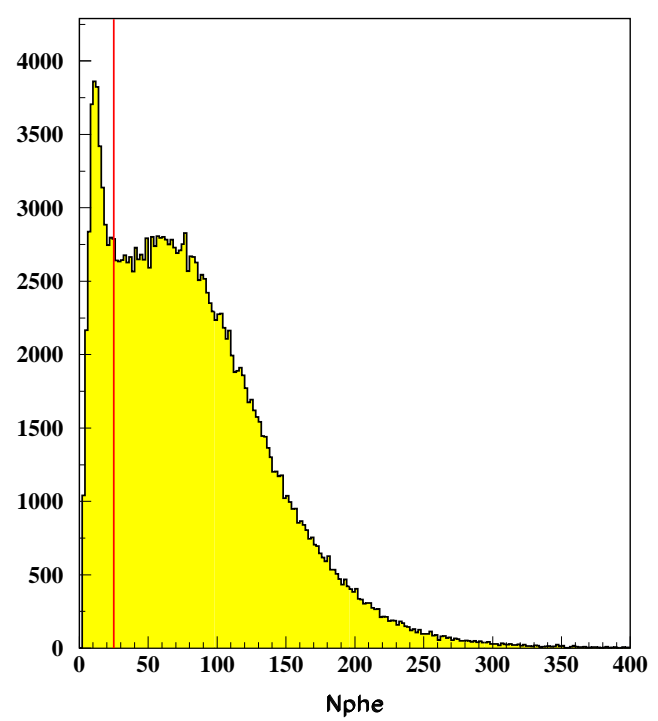

Figure 3.3: CC signal threshold cut using the number of photoelectrons.

energy by creating $e^{+} e^{-}$pairs, thus producing electromagnetic showers. The energy deposited by this method is momentum dependent as can be seen from the left plot of Fig. 3.4.

The momentum of the pions that fire the Cherenkov counters exceeds $2.5 \mathrm{GeV}$, in which case the pions are minimum ionizing. Their deposited energy is independent of the particle momentum. Fig. 3.5 shows the energy deposition in the EC outer layers versus the EC inner layers. The pion characteristic distributions can be seen in both plots. $E_{\text {inner }}>0.06 \mathrm{GeV}$ can effectively remove most of the pions from the electron sample.

\section{EC Sampling Fraction Cut}

Only part of the total electron energy can be measured in the calorimeter because of the interaction of the electrons with the lead layers. The fraction of energy, which the calorimeter is able to measure, is called the sampling fraction (S.F.) and it is a property of the calorimeter, determined from calibration measurements (S.F. 0.29). The deposited energy vs. momentum distribution for electrons is given in Fig. 3.4. 

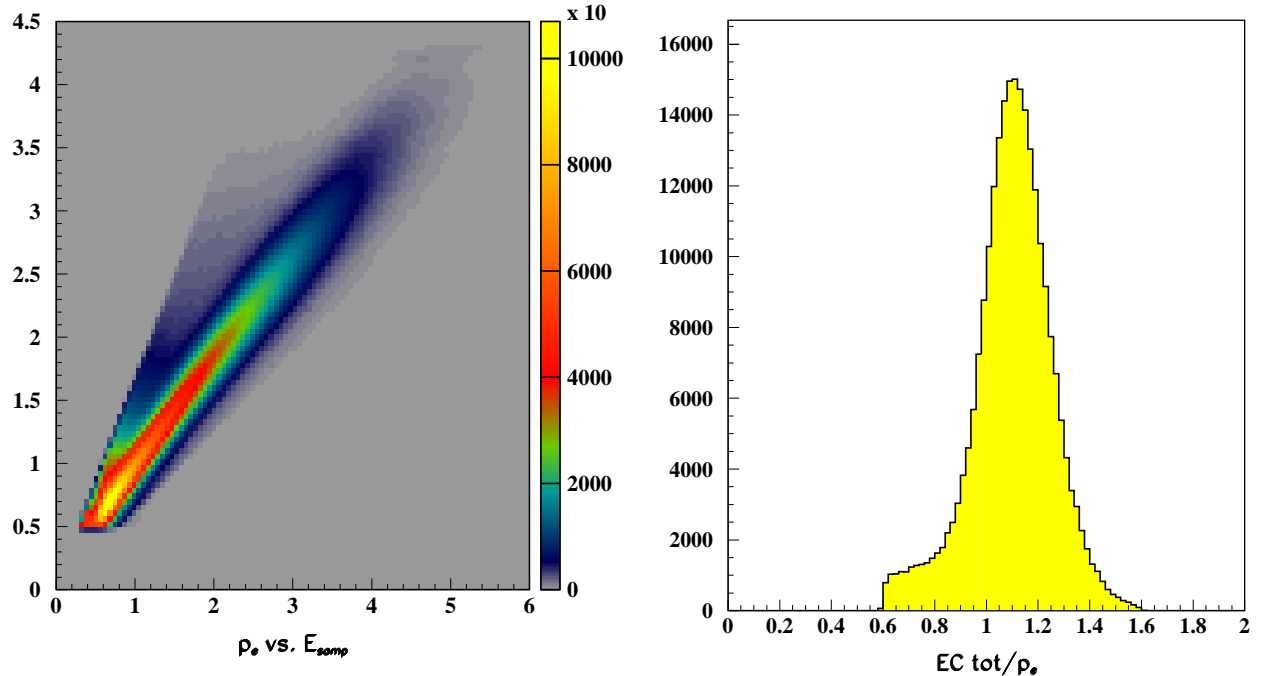

Figure 3.4: EC deposited energy vs. momentum distribution for electrons. The $E_{\text {sample }}$ in this plot is the deposited energy divided by the calorimeter sampling fraction. The right plot shows the $E_{\text {sample }} / p_{e}$ ratio for the electrons.
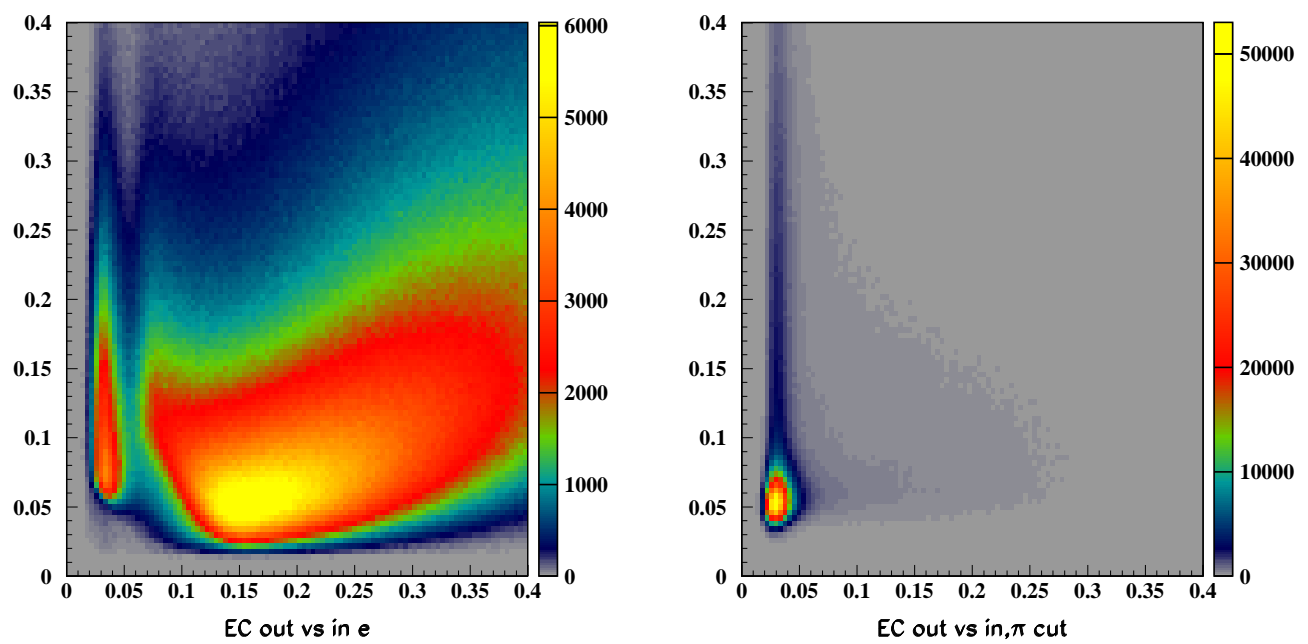

Figure 3.5: Energy deposition in the EC outer layers versus the EC inner layers (left). The same distribution with the pion cut is given on the right plot. The characteristic pion behavior is visible in both plots. 
The $E_{\text {sample }}$ in this plot is the deposited energy divided by the S.F. of the calorimeter. The right plot shows the $E_{\text {sample }} / p_{e}$ ratio for the electrons. Both deposited energy and sampling fraction cuts are omitted in the present analysis for the reasons discussed in Section 3.1.

\section{Trigger Threshold Cut}

The trigger threshold cut is a cut on electron energy near the EC hardware threshold. The electron energy cutoff is not as sharp as ideally expected as a result of amplitude fluctuations. The electron energy threshold according to Ref. [43] is given by:

$$
E_{e}(\mathrm{MeV}) \geq 214+2.47 \times \mid E C_{\text {tot }} \text { threshold }(\mathrm{mV}) \mid .
$$

For the E1F dataset the threshold was set at $170 \mathrm{mV}$, which translates into $640 \mathrm{MeV}$ minimum electron energy. The software cut on energy is removed in the final analysis as discussed in Section 3.1.

\section{Electron $z$-Vertex Cuts}

The electron $z$-vertex cut is applied to ensure that the electrons causing the trigger have an interaction vertex within the liquid-hydrogen target region. The interaction verteces are reconstructed by extrapolating the tracks back to target region and finding the intersection points of each track with the midplane of the same sector in which the track was detected. The midplane of the sector includes the $z$-axis. If the beam is not exactly centered at $(0,0)$, it can result in distortions in reconstructed vertex positions. The reconstructed $z$-vertex distributions are sector dependent, as can be seen from Fig. 3.6. The vertex cut $-29.0 \mathrm{~cm}<z_{e}<-21.5 \mathrm{~cm}$ is applied to electron vertex positions. The vertex

correction routine used here was originally written by Valery Kubarovsky [44] for the E1C analysis and was modified for the E1F dataset. The correction routine uses the beam $x$ and $y$ positions to correct the electron vertex in each sector. These corrections are applied in order to avoid the sector dependent cuts on the vertex position. 


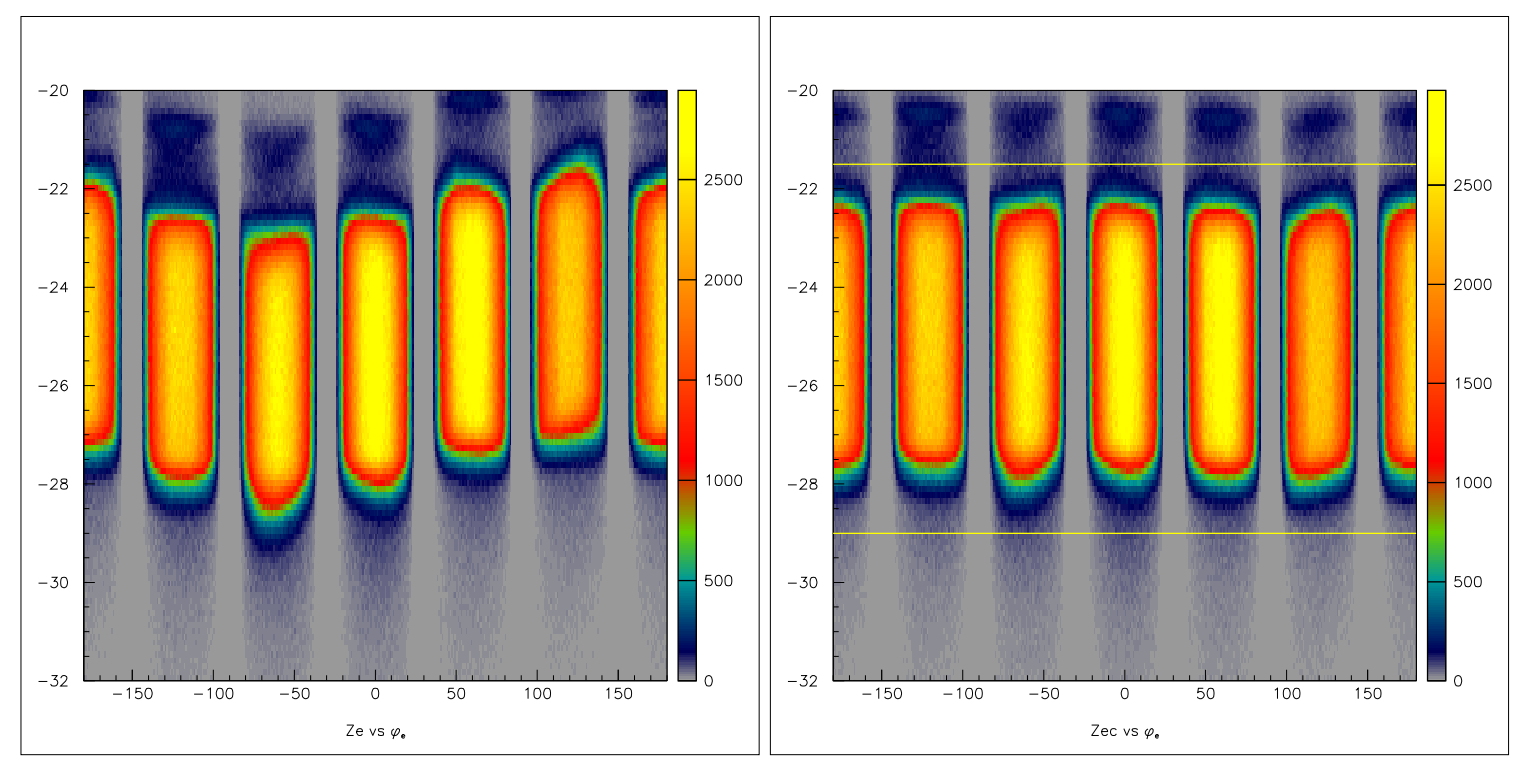

Figure 3.6: $z$-vertex distributions for electrons as a function of $\phi_{e}$. The left plot is before the vertex corrections and the right plot is after the correction. The applied cuts are shown by yellow lines.

\section{Electromagnetic Calorimeter Fiducial Cuts}

Since the electromagnetic calorimeter is used for particle identification, energy measurements and separation of pion background from electrons for energies greater than $2.5 \mathrm{GeV}$ (when the pion Cherenkov radiation threshold is exceeded), it is necessary to ensure that the created electromagnetic shower is fully contained within the EC fiducial volume. The specific cuts were applied to ensure that the shower centroid appears at least $10 \mathrm{~cm}$ away from the $U, V$, and $W$ plane edges. The following cuts were employed by using Stepan Stepanyan's routine [45]:

$$
20 \leq U_{E C} \leq 400 \mathrm{~cm}, V_{E C} \leq 375 \mathrm{~cm}, W_{E C} \leq 410 \mathrm{~cm} \text {. }
$$

Electron $y$ vs. $x$ distributions, projected on the calorimeter surface, before fiducial cuts (top) and after EC fiducial cuts (bottom) are shown in Fig. 3.7. In this analysis the EC is only used for energy measurements and triggering. 


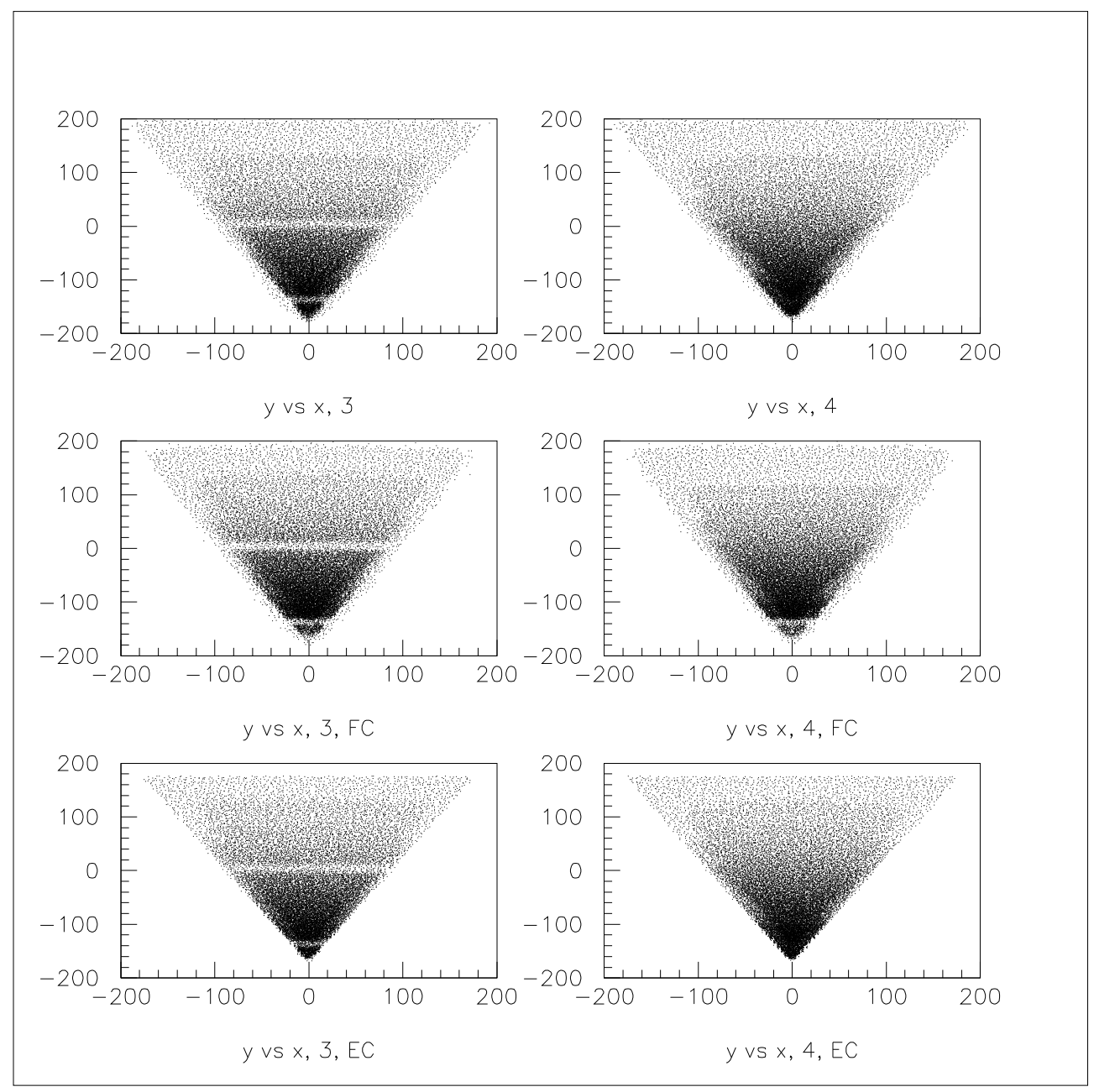

Figure 3.7: $y$ vs. $x$ distributions of electrons projected on the calorimeter surface for sectors 3 and 4 . The position distributions before fiducial cuts (top), after geometrical fiducial cuts (middle) and after EC fiducial cuts (bottom) are shown. 


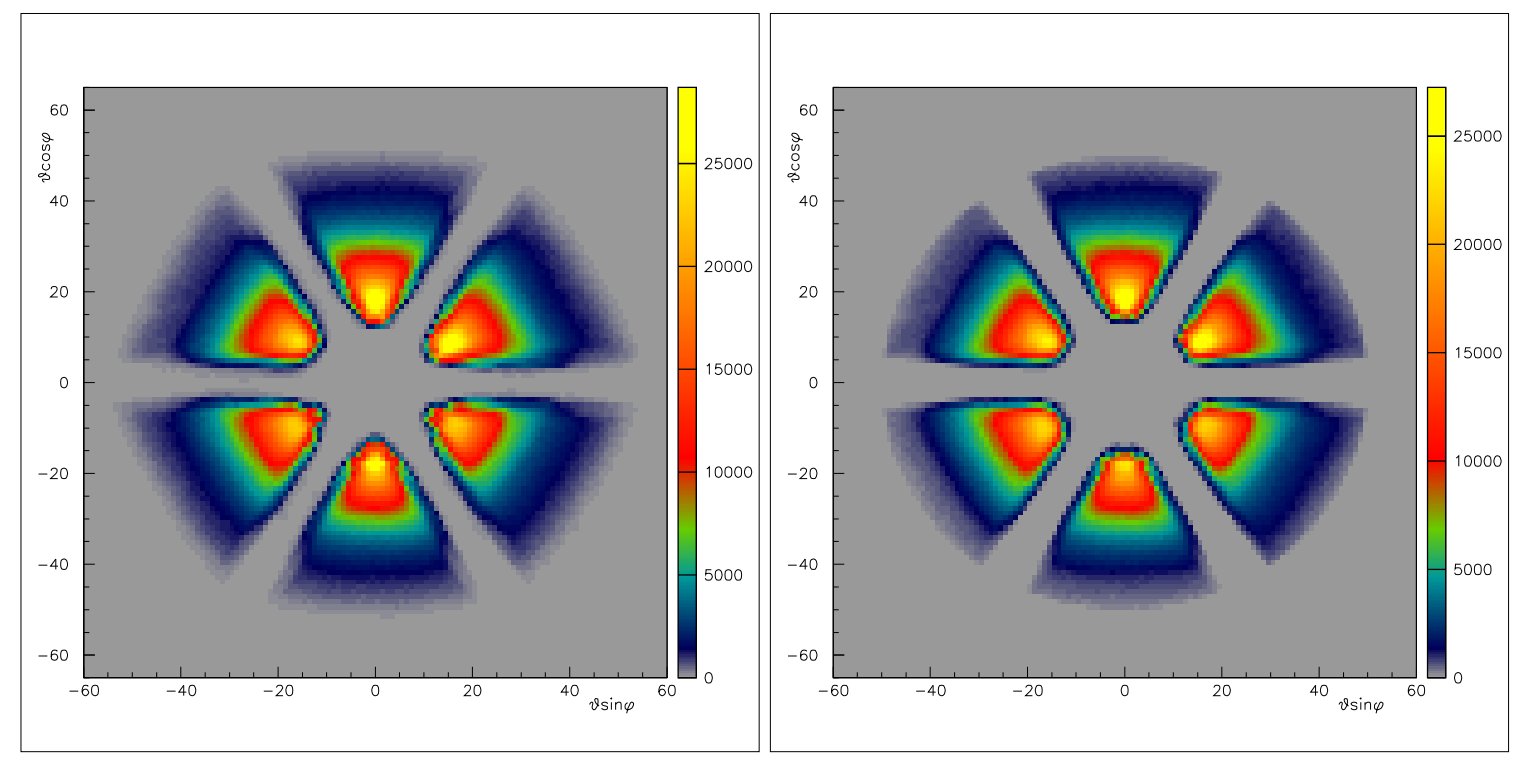

Figure 3.8: The angular coverage $\left(\theta_{e} \cos \phi_{e}\right.$ vs. $\left.\theta_{e} \sin \phi_{e}\right)$ of electrons before (left) and after fiducial cuts (right).

\section{Electron Geometrical Fiducial Cuts}

Electron geometrical fiducial cuts serve the purpose of selecting the flat acceptance regions of CLAS, where the efficiency is large. These cuts are applied to all final-state particles and are momentum dependent. The fiducial volume is specified by applying cuts on the ranges of the polar and azimuthal angles of the electron. Electron geometrical fiducial cuts are defined by the following expressions:

$$
\begin{aligned}
\theta_{\text {min }} & =\theta_{1}+\theta_{2} /\left[\left(p_{e}+p_{0}\right) I_{\max } / I\right], \\
\delta \phi_{e} & =\phi_{0} \sin \left(\theta-\theta_{\text {min }}\right)^{x}, \\
x & =a\left(p_{e} I_{\text {max }} / I\right)^{b} .
\end{aligned}
$$

For the E1F data set, $I=2250$ A so that $I_{\max } / I=1.5$. Only loose fiducial cuts were applied to electrons in this analysis. The list of parameters and their corresponding values are summarized in Table 3.1. The top and the middle plots of Fig. 3.7 show the electron $y$ vs. $x$ distributions projected on the calorimeter surface, before and after the geometrical fiducial cuts. Fig. 3.8 demonstrates the electron angular coverage before 


\begin{tabular}{|r|r|r|}
\hline Parameter & FC Loose & FC Medium \\
\hline$\theta_{1}$ & 9.5 & 11.5 \\
\hline$\theta_{2}$ & 26.0 & 26.0 \\
\hline$p_{0}$ & 0.5 & 0.5 \\
\hline$\phi_{0}$ & 24.0 & 22.0 \\
\hline$a$ & 0.01 & 0.01 \\
\hline$b$ & 1.2 & 1.2 \\
\hline
\end{tabular}

Table 3.1: Parameters used for electron geometrical fiducial cuts in Eq. 3.2. All angles are measured in degrees and momenta in $\mathrm{GeV}$.

and after the geometrical fiducial cuts are applied.

In addition to geometrical fiducial cuts, two dimensional $\theta_{e}-p_{e}$ cuts are applied in order to eliminate the inefficient or dead sections of the DC in each sector.

$$
\theta_{e}^{ \pm}\left(p_{e}\right)=\theta_{0}^{ \pm}\left[1-\exp \left(-b^{ \pm} \cdot\left(p_{e}-c^{ \pm}\right)\right)\right]
$$

In Eq. 3.3, \pm refers to the upper and lower limits on $\theta_{e}$. The parameters $\theta_{0}, b$, and $c$ are defined separately for each sector and listed in Table B.1 of Appendix B. Fig. 3.9 demonstrates the effects of these cuts for electrons in sector 3.
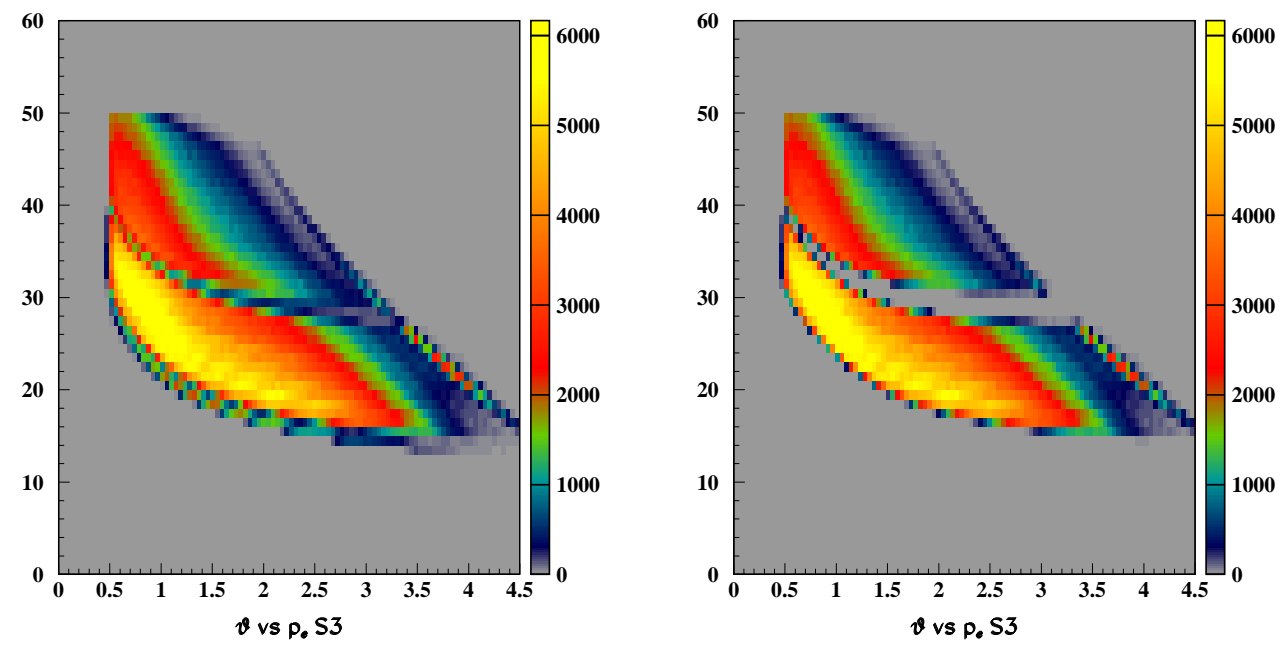

Figure 3.9: $\theta_{e}$ vs. $p_{e}$ for Sector 3. 


\subsection{Hadrons}

Hadrons are required to have a valid track in the DC corresponding to a positively charged particle and a hit in the time of flight system that coincides in time. In this analysis the hadrons are identified by a timing cut instead of momentum-dependent mass cuts. For this purpose the time difference $\left(\Delta t=t_{1}-t_{2}\right)$ is calculated between the measured time, $t_{1}$, it took the particle to travel from the interaction vertex position to the SC paddle of the TOF system and the time, $t_{2}$, it takes the particle with an assumed mass to travel the same distance. Here $t_{1}$ is given by the $\mathrm{SC}$ and can be expressed as:

$$
t_{1}=\frac{d}{c \beta_{1}}
$$

where $d$ is the path length of the particle from the vertex to the SC paddle, determined by the tracking system, $c$ is the speed of light and $\beta_{1}$ is the $v_{1} / c$ ratio. The velocity $\beta_{1}$ is actually measured by inverting Eq. 3.4. The mass $m_{1}$ of the hadron is calculated from the velocity and the momentum by:

$$
m_{1}=\frac{p}{\gamma c \beta_{1}}
$$

where $p$ is the hadron momentum as determined by the DC and $\gamma=\frac{1}{\sqrt{1-\beta_{1}^{2}}}$. The time $t_{2}$ is calculated by:

$$
t_{2}=\frac{d}{c \beta_{2}}
$$

where $\beta_{2}$ is now given by the following expression:

$$
\beta_{2}=\frac{p}{\sqrt{\left(m_{2} c\right)^{2}+p^{2}}}
$$

Here $m_{2}$ is the assumed particle mass. After substituting $t_{1}$ and $t_{2}$ into $\Delta t$, it can be reduced to:

$$
\Delta t=t_{1}\left(1-\sqrt{\frac{p^{2}+\left(m_{2} c\right)^{2}}{p^{2}+\left(m_{1} c\right)^{2}}}\right) .
$$


For all positive tracks, $\Delta t$ is calculated three times with an assumed particle mass of a pion, kaon and proton. The mass that gives the smallest $\Delta t$, is assigned to the hadron. Fig. $3.10 \mathrm{a}$ ) and b) show the minimum $\Delta t$ vs. $p$ distributions for kaons and protons before any cuts. The finite bands at $\pm 2 \mathrm{~ns}$ and $\pm 4 \mathrm{~ns}$ in Fig. $3.10 \mathrm{~b}$ ) are due to accidental events from different beam bunches of the accelerator. Fig. $3.10 \mathrm{c}$ ), d) show the same distributions for kaons and protons after applying the $\Lambda$ missing-mass and $\pi$ missingmass-squared cuts (Section 3.3). The application of these cuts effectively removes the accidental coincidences and most of the background in the kaon distribution, which consists of pions and protons misidentified as kaons. The timing method of hadron identification insures that every track corresponding to a positively charged particle is identified. It gives better results than the momentum-dependent mass cut because it is almost momentum independent while the mass cut method strongly depends on particle momentum. As $\beta \rightarrow 1$, the pion, kaon, and proton bands start overlapping thereby worsening the mass resolution.
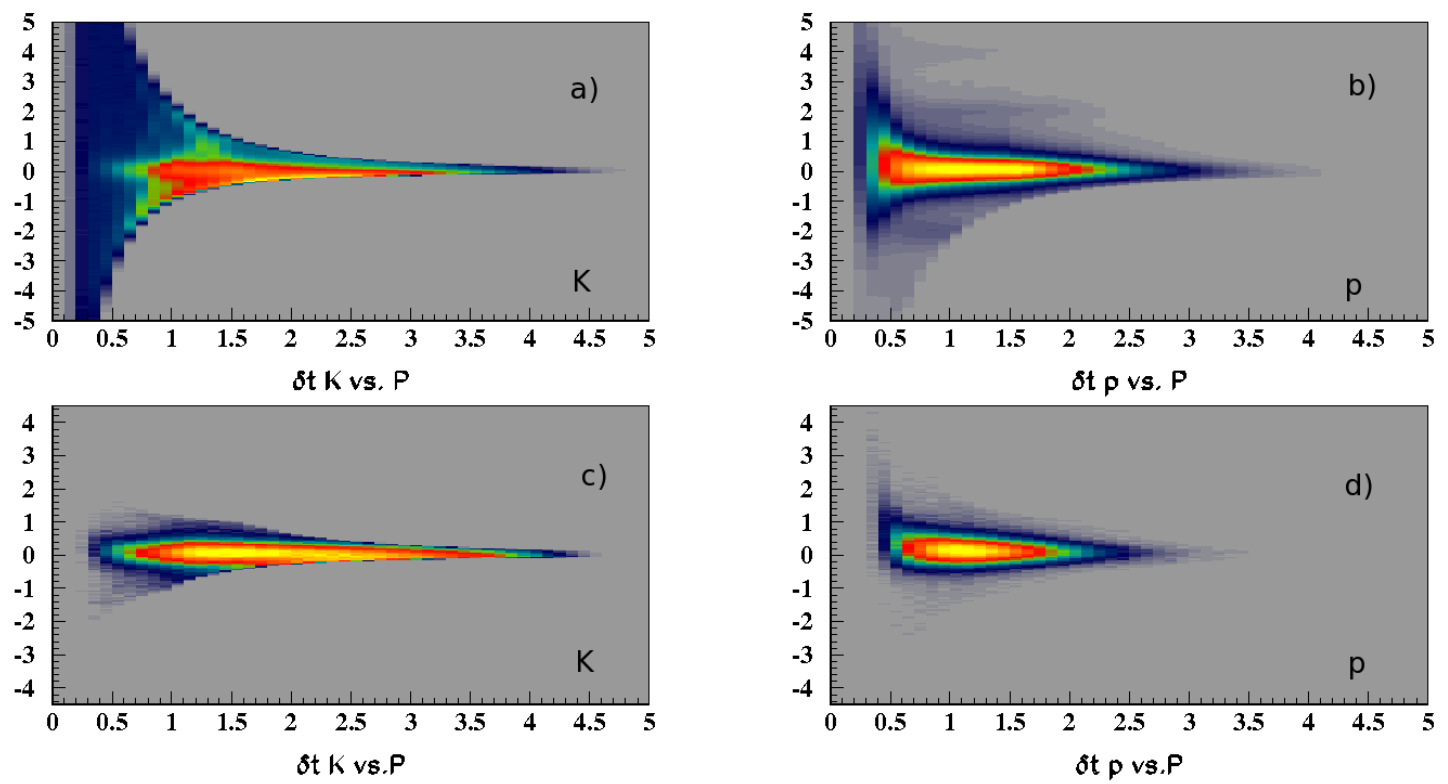

Figure 3.10: Minimum $\Delta t$ vs. $p$ distributions for kaons and protons. a) and b) show the distributions without any cuts. c) and d) show the same distributions for kaons and protons after applying the $\Lambda$ missing-mass and $\pi$ missing-mass cuts. 


\section{Hadron $z$-Vertex Cuts}

A $z$-vertex position cut is necessary also for kaons to make sure that they originate from the target region. Vertex positions are corrected by the same routine as for the electrons. The $z$-vertex distributions along with the applied cuts are shown in Fig. 3.11. A cut of $-29 \mathrm{~cm}<z_{K}<-21 \mathrm{~cm}$ is applied on the reconstructed kaon vertex positions. Since the final state protons are coming from the $\Lambda$ decay, which can be outside of the target region, no $z$-vertex cut is applied to protons.

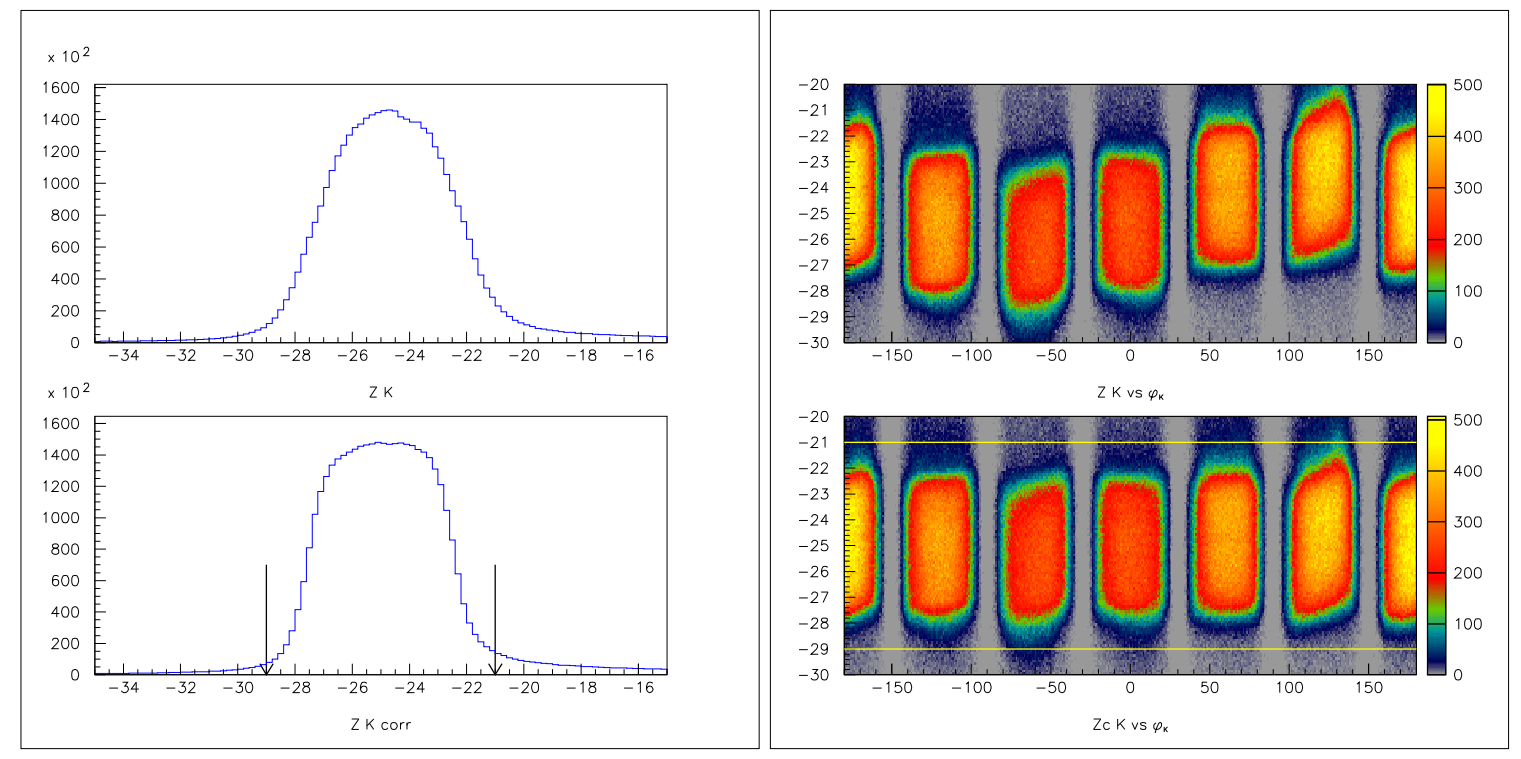

Figure 3.11: $z$-vertex distribution for kaons before and after corrections. The applied cuts are shown by arrows and yellow lines.

\section{Hadron Geometrical Fiducial Cuts}

Hadron fiducial cuts are employed in order to exclude the low-acceptance regions of CLAS spectrometer. These cuts are applied to both kaons and protons and are momentum dependent. The fiducial volume is again specified by applying cuts on the ranges of polar and azimuthal angles of the hadrons. The hadron fiducial cuts are defined by 


\begin{tabular}{|r|r|r|}
\hline Parameter & FC Loose & FC Medium \\
\hline$a$ & 0.22 & 0.22 \\
\hline$b$ & 0.15 & 0.15 \\
\hline$\theta_{1}$ & 4.0 & 5.0 \\
\hline$\theta_{2}$ & 20.0 & 20.0 \\
\hline$c$ & 8.0 & 8.0 \\
\hline$d$ & 15.0 & 15.0 \\
\hline$\phi_{0}$ & 32.0 & 31.0 \\
\hline
\end{tabular}

Table 3.2: Parameters used for hadron geometrical fiducial cuts in Eq. 3.9. All angles are measured in degrees and momenta in $\mathrm{GeV}$.

the following expressions:

$$
\begin{aligned}
x & =a\left(p_{h} I_{\max } / I\right)^{b} \\
\theta_{\min } & =\theta_{1}+\theta_{2}\left(1-\left(\left(p_{h} / c\right) I_{\max } / I\right)\right)^{d}, \\
\delta \phi_{h} & =\phi_{0} \sin \left(\theta-\theta_{\min }\right)^{x} .
\end{aligned}
$$

The cuts applied for hadrons are loose cuts as well, with the list of parameters summarized in Table 3.2. Fig. 3.12 shows the kaon angular distributions before and after the fiducial cuts are applied. For the E1F data set, $I=2250$ A so that $I_{\max } / I=1.5$.

Two dimensional $\theta_{h}-p_{h}$ cuts are also applied to hadrons in order to eliminate inefficient or dead areas of DC. The form of the cuts is again given by Eq. 3.3. The parameters are summarized in Table B.2 of Appendix B. Fig. 3.13 demonstrates the effects of these cuts for kaons in sector 3.

\section{Bad Paddle Removal}

One additional cut that is applied to all final-state particles is the SC bad paddle removal. During the course of the E1F run some of the SC paddles proved to be inefficient or dead. The events from these inefficient paddles are removed from the analysis. They can be identified from the hadron mass vs. paddle number distributions as shown in Fig. 3.14. Discontinuities and sharp transitions in this figure are the result of inefficient 


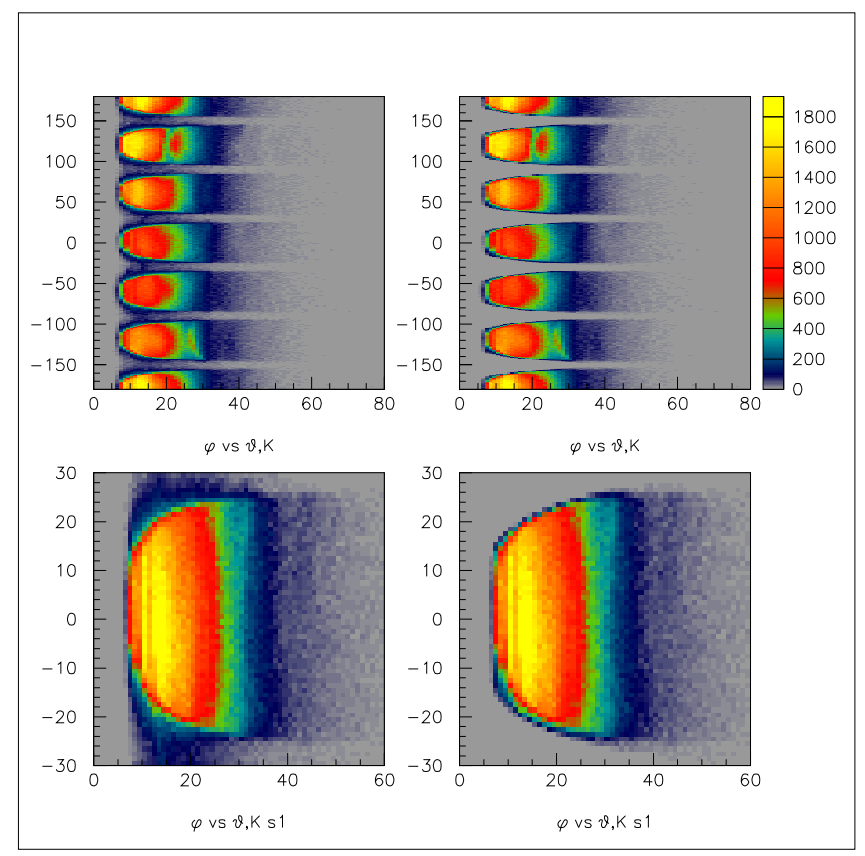

Figure 3.12: Kaon $\theta_{K}$ vs. $\phi_{K}$ distributions for all sectors (top) and for the Sector 1 alone (bottom) before (left) and after (right) the fiducial cuts.
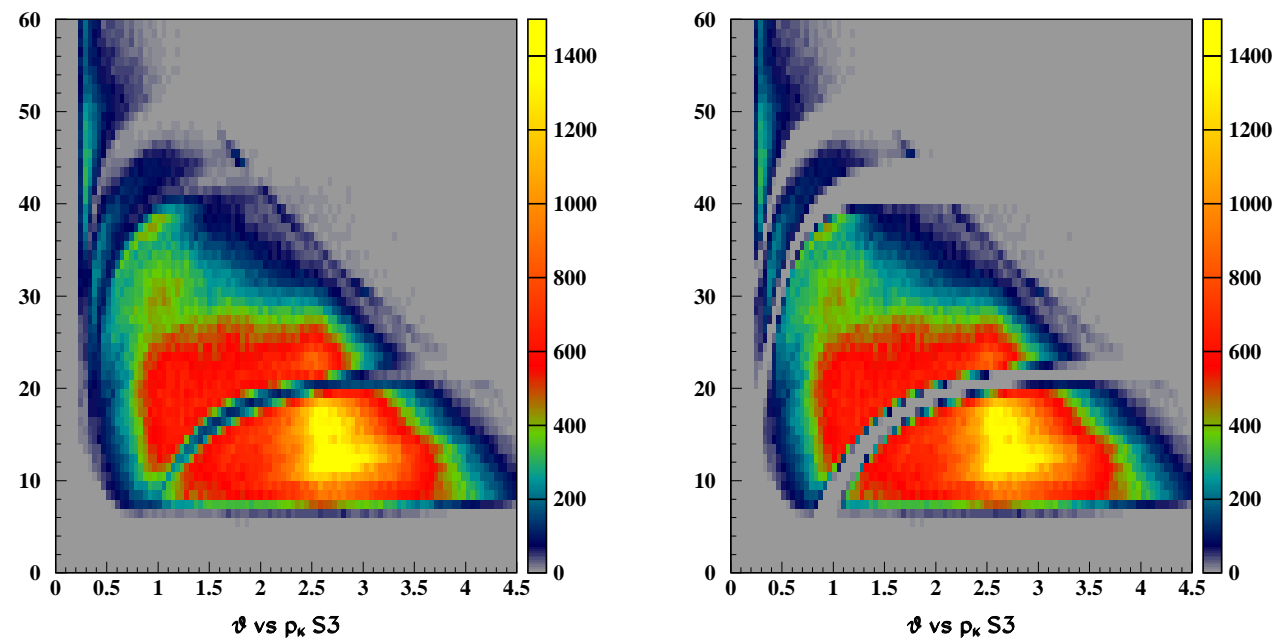

Figure 3.13: Kaon $\theta_{K}$ vs. $p_{K}$ for Sector 3. 


\begin{tabular}{|r|r|}
\hline Sector & Bad Paddles \\
\hline S1 & 24 \\
\hline S2 & $16,28,38$ \\
\hline S3 & $2,11,24,27,28,40$ \\
\hline S4 & $2,19,30,34$ \\
\hline S5 & $2,18,20,34,40$ \\
\hline S6 & $1,18,40$ \\
\hline
\end{tabular}

Table 3.3: List of removed SC paddles.

or dead paddles. The list of the removed paddles for each sector is summarized in Table 3.3.

\subsection{Hyperon Identification}

In this analysis the final state hyperons are identified by utilizing the missing mass technique. Namely, using energy and momentum conservation, the missing energy and momentum are calculated from the $e^{\prime} K^{+}$final state according to Eq. 1.8. The missing mass distribution, before any physics cuts, is shown in Fig. 3.15 a). The $n$ peak comes from the $e p \rightarrow e^{\prime} n \pi^{+}$reaction, where the pion is misidentified as a kaon. Once the required energy threshold is exceeded, the higher mass hyperons are also produced as can be seen from in Fig. 3.15 a). The presence of a proton in the final state, which comes from the $\Lambda$ decay, reduces the $n$ peak and some of the background (see Fig. 3.15 b)). Since the $p$ is also present in the higher mass hyperon decays, their contributions in the $\Lambda$ missing mass distribution cannot be fully eliminated by $p$ requirement alone. The strongest cut to identify the final state of interest is the $\pi^{-}$missing-mass-squared cut $\left(M M^{2}\left(e^{\prime} K^{+} p\right)\right)$ reconstructed from the $e^{\prime} K^{+} p$ final state (Eq. 1.9). The correlation $M M^{2}\left(e^{\prime} K^{+} p\right)$ vs. $M M\left(e^{\prime} K^{+}\right)$is shown in Fig. 3.16 a). Fig. 3.16 b) and c) are the projections of the correlation plot on the respective axes. The red lines in Fig. 3.16 b) show the corresponding cuts applied for $\Lambda$ selection in the final analysis. The applied cut $-0.02<M M^{2}\left(e^{\prime} K^{+} p\right)<0.07 \mathrm{GeV}^{2}$ includes $\pi^{-}$from $\Lambda \rightarrow p \pi^{-}$, and $\pi^{-} \gamma$ from $\Sigma^{0} \rightarrow \Lambda \gamma$ decays. The photons are in the shoulder on the high mass side of the $\pi^{-}$ 

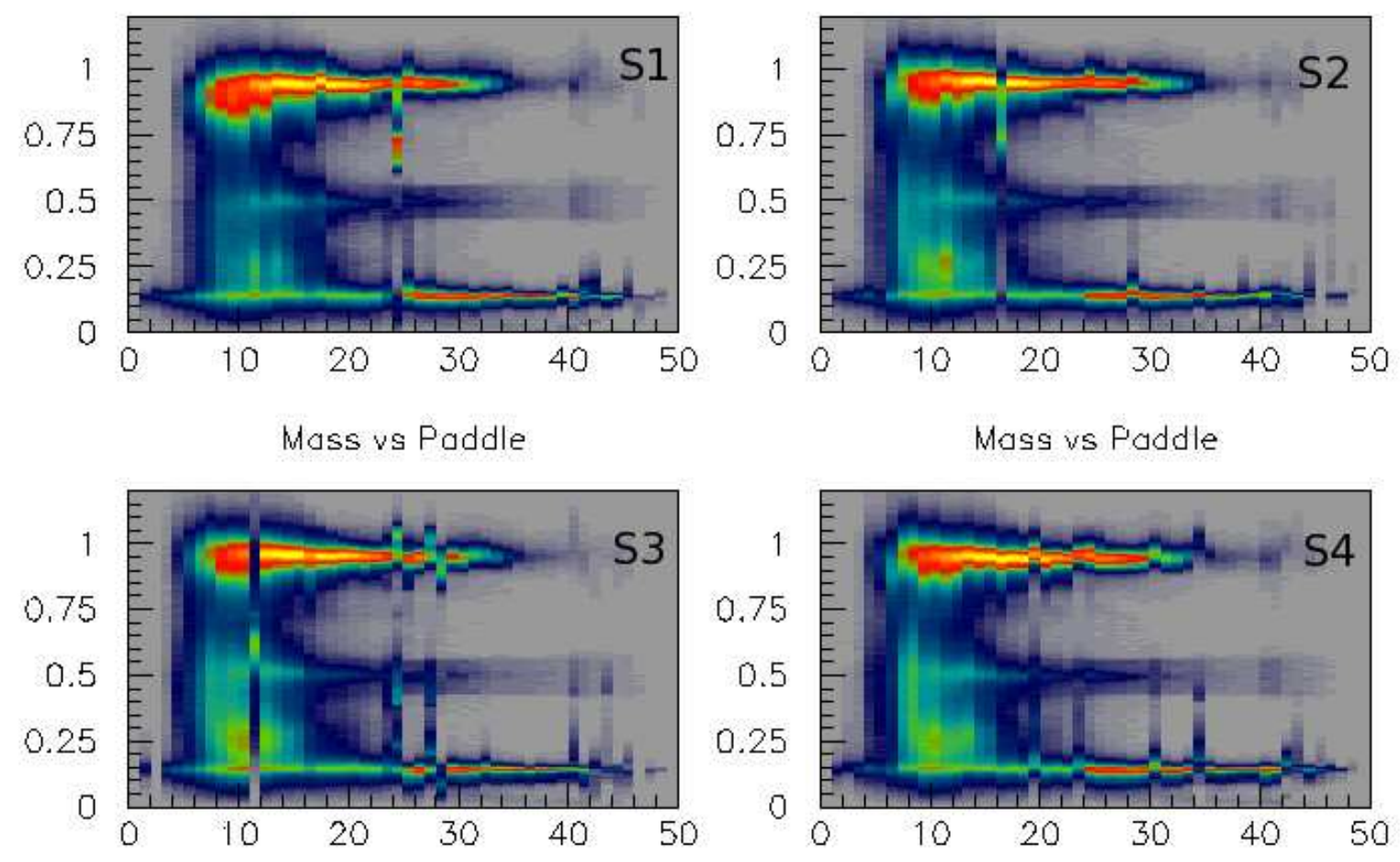

Mass vs Paddle
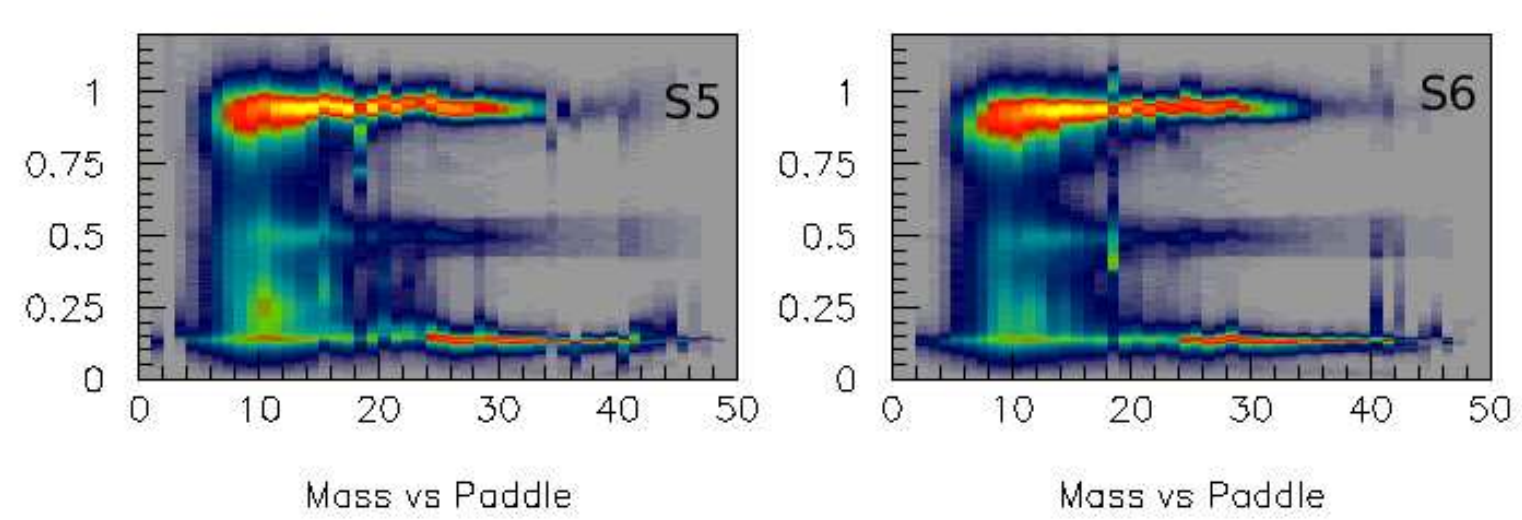

Figure 3.14: Hadron mass vs. SC paddle number for each sector. Bad paddles can be identified from these plots. 

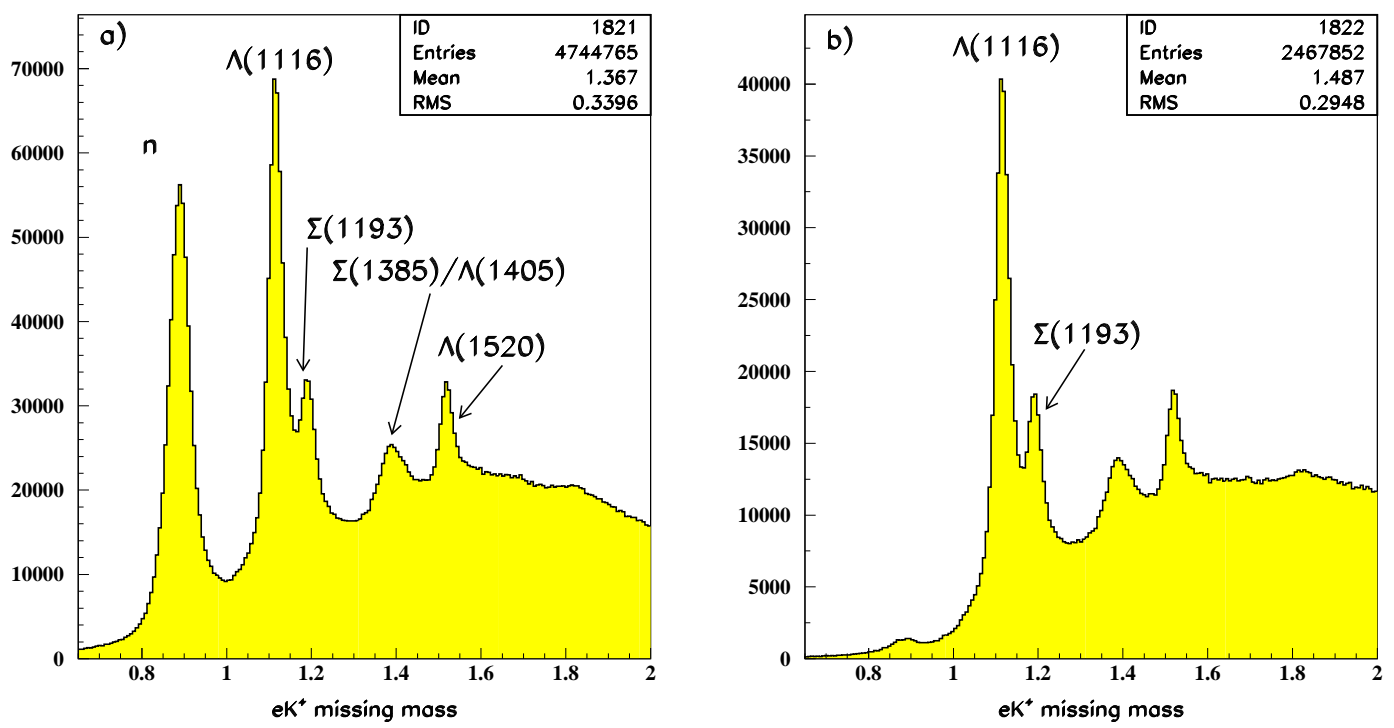

Figure 3.15: $\Lambda$ missing mass distributions a) before any cuts, b) after $p$ presence requirement.
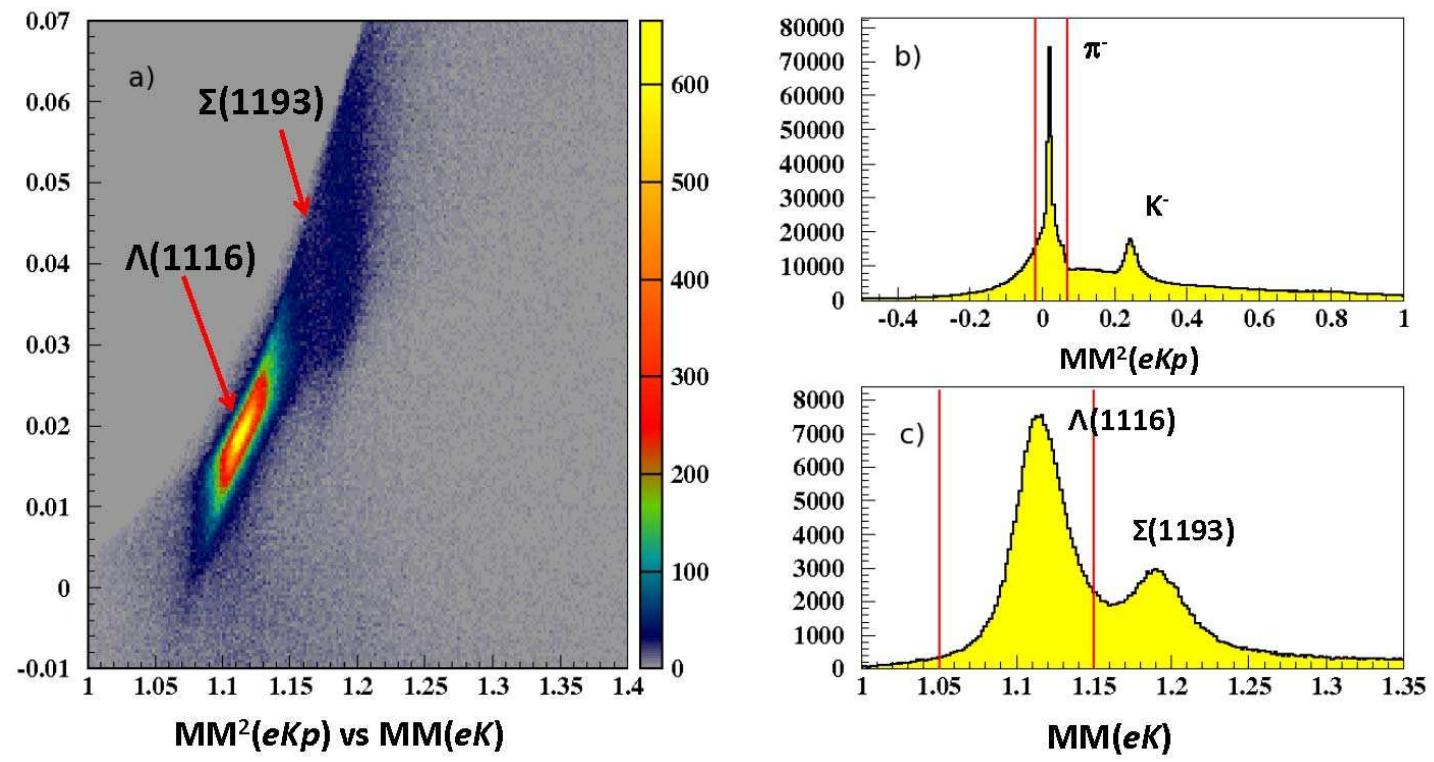

Figure 3.16: a) Reconstructed meson missing mass squared vs. baryon missing mass correlation, b) Meson missing mass squared distribution. The red lines show the applied cuts on $\pi$ missing mass squared. c) baryon missing mass distribution after applying $\pi$ missing-mass-squared cut. The red lines in this plot show the missing mass range over which the background subtracted yields are integrated for the final $\Lambda$ sample selection. 
peak. Shown in Fig. 3.16 c) is the missing mass distribution, after applying the $-0.02<$ $M M^{2}\left(e^{\prime} K^{+} p\right)<0.07 \mathrm{GeV}^{2}$ cut. Note that no $\Lambda$ missing mass cuts are applied directly. Instead, the background subtracted yields are integrated over the missing mass range from $1.05 \mathrm{GeV}$ to $1.15 \mathrm{GeV}$ (red lines in Fig. $3.16 \mathrm{c}$ )). The resolution between the $\Lambda(1116)$ and $\Sigma^{0}$ is not very sharp because of the low magnetic field used during the experiment. The upper limit of $M M^{2}\left(e^{\prime} K^{+} p\right)\left(0.07 \mathrm{GeV}^{2}\right)$ is extended in order to include all $\Sigma^{0}$. It is crucial to have enough statistics of $\Sigma^{0}$ 's to bin and fit the $M M\left(e^{\prime} K^{+}\right)$ distributions in order to eliminate the $\Sigma^{0}$ contamination from underneath the $\Lambda$ peak, along with the pion background in each kinematic bin. The background subtraction procedure is discussed in detail in Section 4.4. 
CHAPTER 4

\section{Analysis}

\subsection{Cooking and Data Reduction}

During the experiment the data acquisition system writes the data files to a tape silo. The acquired data for every run period need to be processed or "cooked" before any full scale analysis can be performed on these data. All subsystems of CLAS need to be calibrated separately by using a small subset of the data in order to get high-quality and publishable results. The calibration constants for each CLAS component are saved in the calibration database. During the cooking procedure, cooking executables first check if any event fragments have been lost during the writing process, then access the calibration database, read and apply the calibration constants and create all necessary banks for the analysis. Different monitoring programs create histogram files that can be used for updating the database and for checking the quality of the data. After the cooking procedure is complete, the more user friendly ntuples and root trees are created, which can be used for the full scale analysis.

The newly cooked files are usually very large in size. Since different run groups usually concentrate on analyzing specific reactions, it is much more convenient to filter the data and preselect the events with likely particle candidates. The filtering scripts skim through the data and keep only those events that are passing some loose particle identification cuts. The filtering process is especially useful for low cross-section channels, like kaon electroproduction. For the E1F data set, several skimming filters such as $e, e^{\prime} K^{+}, e^{\prime} K^{+} p$ were used. In present analysis, the $e$ skimmed set was used for

momentum corrections and background studies, while the $e^{\prime} K^{+} p$ skimmed set, preselected for $\mathrm{K}^{+}$and $p$ candidates, was used for polarization extraction. The size of the $e^{\prime} K^{+} p$ skimmed data set was considerably reduced, since all events with less than three particles in them, or with no valid kaon or proton candidates, were ignored. The $e^{\prime} K^{+} p$ 
skimmed file sizes are only $10 \%$ of that of the $e$ skimmed files. The $e$-filter requires the electron candidate to have a negative charge, a valid track, a momentum greater than $0.5 \mathrm{GeV}$ and satisfy a very loose sampling fraction cuts. Kaon and proton candidates are required to have a positive charge, a valid track and have less than 5\% difference between the measured and calculated $\beta$ values $\left(\Delta \beta=\left|\beta^{\text {meas }}-\beta^{\text {calc }}\right|<0.05\right)$, where $\beta^{\text {calc }}$ is calculated by using the particle momentum as measured by the DC and the nominal particle mass. Full details of the data processing procedures can be found in Ref. [42].

\subsection{Binning}

The kinematics of the electron scattering reaction are uniquely determined by four independent variables. The variables employed in this analysis are the invariant energy, $W$, of the intermediate hadronic state, the transferred momentum $Q^{2}$, the kaon scattering angle in the center of momentum frame $\left(\theta_{K}^{C M}\right)$ and the relative angle $\phi_{K}$ between the electron scattering and the hadron production planes as shown in Fig. 1.1. The kinematic dependences of these variables are shown in Fig. 4.1. The bin widths are chosen to have approximately equal statistical uncertainties in each kinematic bin. The binning used for this analysis is tabulated in Table 4.1.
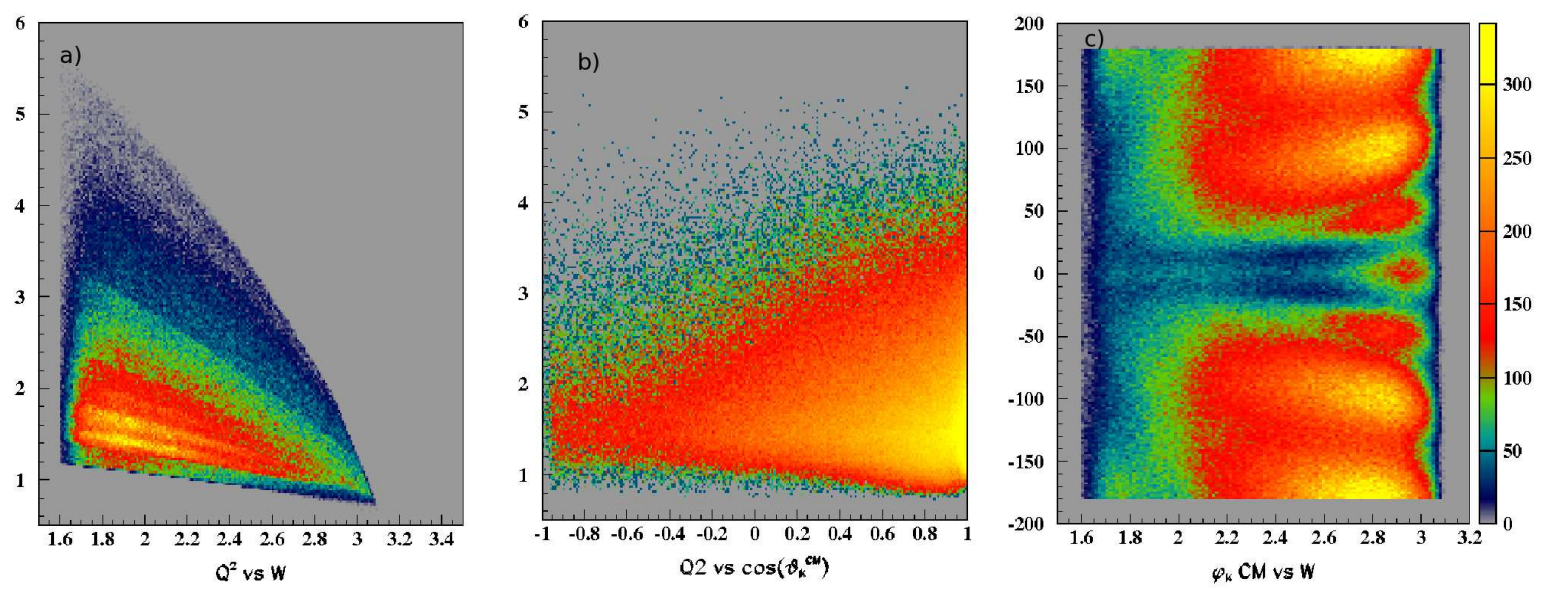

Figure 4.1: Kinematic distributions a) $Q^{2}$ vs. $W$, b) $Q^{2}$ vs. $\cos \theta_{K}^{C M}$, and c) $\phi_{K}^{C M}$ vs. $W$. 
The $Q^{2}$ dependence of the polarization was extensively studied. Details are given in Section 4.8. The results showed a flat $Q^{2}$ dependence for different $W$ bins, which allows one to sum the data over $Q^{2}$ without losing any valuable information. The data are also integrated over $\phi_{K}$, since there are not enough statistics to bin the data. The result of this integration leads to vanishing longitudinal and transverse induced polarization components, as discussed in Section 1.3. The integration over $Q^{2}$ and $\phi_{K}$ greatly improve the statistics in each kinematic bin.

\begin{tabular}{|r|r|r|r|}
\hline Variable & Range & \# of bins & Bin Width \\
\hline \hline $\cos \theta_{K}^{C M}$ & $(-1.0,0.0)$ & 2 & 0.5 \\
& $(0.0,1.0)$ & 5 & 0.2 \\
\hline$W$ & $1.6-2.1 \mathrm{GeV}$ & 20 & $25 \mathrm{MeV}$ \\
& $2.1-2.7 \mathrm{GeV}$ & 12 & $50 \mathrm{MeV}$ \\
\hline
\end{tabular}

Table 4.1: Binning for the polarization studies.

\subsection{Momentum Corrections}

The momentum corrections for electrons and hadrons are performed in order to correct for DC misalignments and inaccuracies in the magnetic field maps. Two separate groups worked on momentum corrections for the E1F data set. Both methods are described in detail in the next few sections. The FIU group used the momentum correction method developed by D.S. Carman for the E1-6 analysis [46]. In this approach the polar angles, as measured by CLAS, are assumed to be correct. The electron skimmed data were used for the momentum correction analysis. The differences between the measured momenta and the momenta calculated from the kinematics were determined and then applied as corrections. Elastic $e p \rightarrow e p$ scattering, $e p \rightarrow e^{\prime} \pi^{+} n$ and $e p \rightarrow e^{\prime} K^{+} \Lambda$ reactions were used to perform the corrections. Overall the momentum corrections are about $1 \%$ for electrons and $1.5 \%$ for hadrons.

Marco Mirazita from INFN developed a different method for the E1F momentum corrections [47]. In this method, the polar angles are corrected first, then the corrected 
angles are used to correct the magnitude of the momentum. To test the $e^{-}$and hadron momentum corrections, the hyperon missing mass spectra are calculated from $e p \rightarrow$ $e^{\prime} K^{+} \Lambda$ reaction using both sets of corrections.

\section{FIU Electron Corrections}

The elastic $e p \rightarrow e p$ scattering is used for electron momentum corrections. Elastic events are identified by requiring both the electron and the proton to be detected by CLAS detector. Additional cuts requiring $W<1.025 \mathrm{GeV}$ and missing mass $\left|M M^{2}(e p)\right|<$ $0.0015 \mathrm{GeV}^{2}$ are applied to clean up the elastic channel. The specific kinematics of elastic scattering requires coplanarity of the scattered particles. This requirement is satisfied by applying a $\left|\phi_{e}-\phi_{p}-\pi\right|<1^{\circ}$ cut. The electron momentum $p^{\text {calc }}$ is calculated by using the beam energy, $E_{\text {beam }}$, which is assumed to be known, and the scattering angle, $\theta_{e}$, as measured by CLAS:

$$
p^{\text {calc }}=\frac{E_{\text {beam }}}{1+\left(2 E_{\text {beam }} \sin ^{2} \frac{\theta_{e}}{2}\right) / M_{p}} .
$$

In this method, first, the two dimensional histograms of $d p=p^{\text {meas }}-p^{\text {calc }}$ vs. $\theta_{e}$ and $\phi_{e}$ are created. As an example, $d p$ vs. $\phi_{e}$ is plotted in Fig. 4.2. These histograms are converted into 1-dim profiles by using the PAW hbprof feature. This feature calculates the average value of $Y$ for each $X$ bin and puts it into a profile histogram with corresponding statistical uncertainties. These profile histograms are then read into vectors and are used for the corrections as follows:

$$
p^{\text {corrected }}=p^{\text {meas }}-\operatorname{vphe}\left(\operatorname{bin}_{\phi}\right)-v \text { the }\left(\operatorname{bin}_{\theta}\right)-\operatorname{vpher}\left(\operatorname{bin}_{\phi}\right)-v \operatorname{ther}\left(\operatorname{bin}_{\theta}\right) .
$$

For each sector the vectors vphe, vthe, vpher and vther are defined separately. The vectors vphe and vpher correspondingly remove the $\phi$ dependence and vthe and vther remove the $\theta$ dependence of $d p$. Fig. 4.3 shows the profile histograms before and after the corrections are applied. The ranges of the kinematic variables are divided into $4^{\circ}$ 


\begin{tabular}{|r|r|r|r|}
\hline Variable & Range & Width & Number of Bins \\
\hline$\theta_{e}$ & $12^{\circ} \rightarrow 40^{\circ}$ & $4^{\circ}$ & 7 \\
\hline$\phi_{e}$ & $-22^{\circ} \rightarrow 22^{\circ}$ & $4^{\circ}$ & 11 \\
\hline
\end{tabular}

Table 4.2: Binning of the electron $\theta_{e}$ and $\phi_{e}$ variables.

\begin{tabular}{|r|r|r|r|r|}
\hline Sector & $W_{e}[\mathrm{MeV}]$ & $\sigma[\mathrm{MeV}]$ & $W_{e}^{\text {corr }}[\mathrm{MeV}]$ & $\sigma^{\text {corr }}[\mathrm{MeV}]$ \\
\hline \hline S1 & 947.3 & 40.8 & 937.2 & 34.6 \\
\hline S2 & 947.7 & 36.2 & 937.4 & 32.7 \\
\hline S3 & 935.2 & 35.5 & 937.5 & 32.6 \\
\hline S4 & 964.9 & 30.9 & 937.5 & 29.9 \\
\hline S5 & 937.8 & 34.8 & 937.5 & 33.1 \\
\hline S6 & 903.4 & 48.8 & 936.4 & 48.8 \\
\hline
\end{tabular}

Table 4.3: Summary of centroids and widths of $W$ distributions before and after the electron momentum corrections. The expected centroid values are supposed to be around the proton mass of $938 \mathrm{MeV}$.

bins as shown in Table 4.2. The process is iterative. In order to get rid of the residual dependencies, four iterations are performed for the electron corrections. Fig. 4.4 shows the fractional momentum correction vs. $p_{e}$. From the plots it can be seen that the momentum corrections are on the order of $1 \%$.

To check the $e^{-}$momentum corrections, $W$ is calculated using the corrected $p_{e}$ momentum by:

$$
W_{e}=\sqrt{M_{p}^{2}+2\left(E_{\text {beam }}-p_{e}\right) \cdot M_{p}-Q_{e}^{2}},
$$

where $Q_{e}^{2}$ is calculated only using the electron scattering angle, the corrected momentum and the beam energy as:

$$
Q_{e}^{2}=2 \cdot E_{\text {beam }} \cdot p_{e} \cdot\left(1-\cos \theta_{e}\right) .
$$

The summary of the centroids and the widths of the $W$ distributions before and after the electron momentum corrections is given in Table 4.3. The results show good improvement in the peak width and are centered around the proton mass as expected for elastic events. 


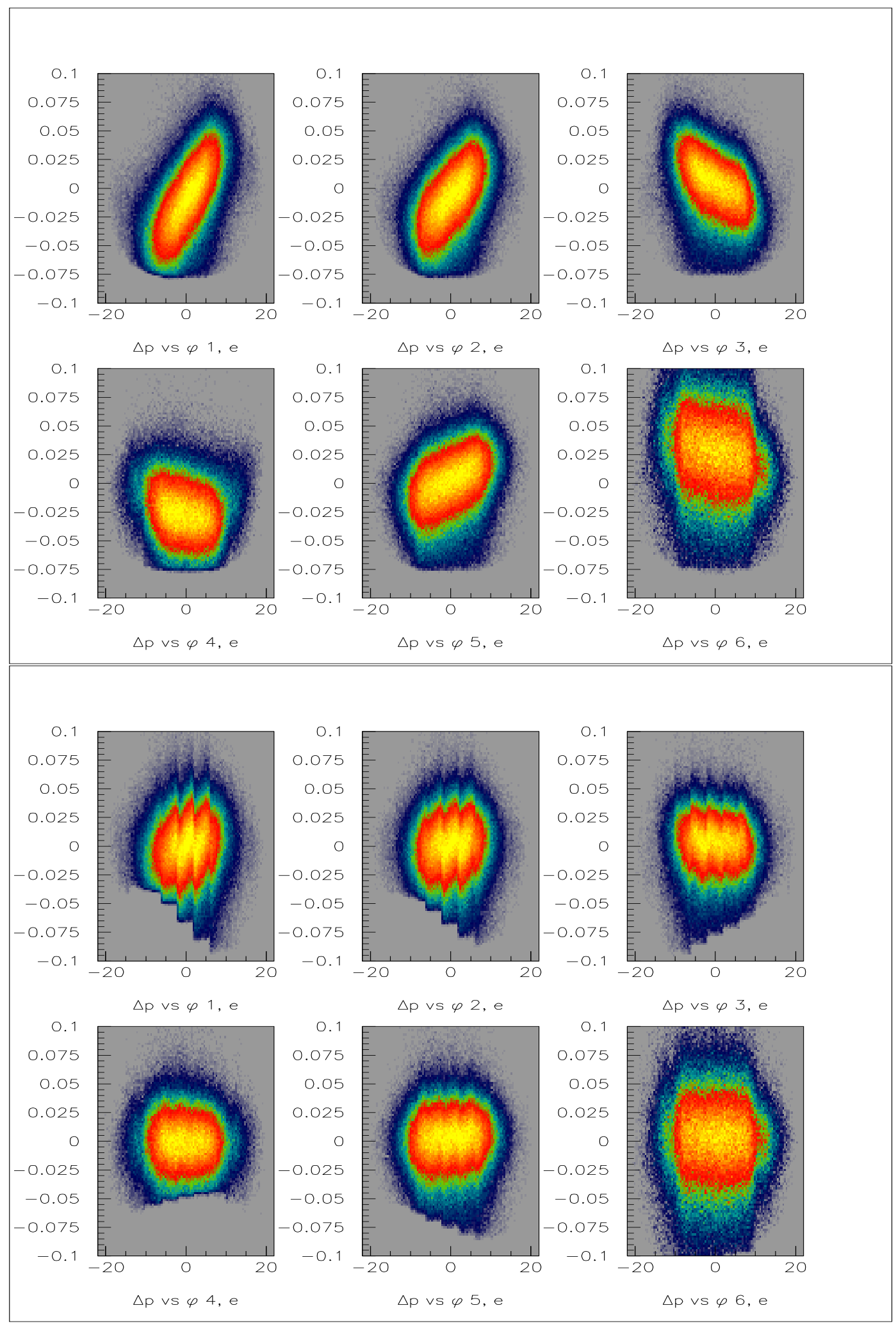

Figure 4.2: Electron $d p$ vs. $\phi_{e}$ distributions for all sectors before (top) and after (bottom) corrections. 

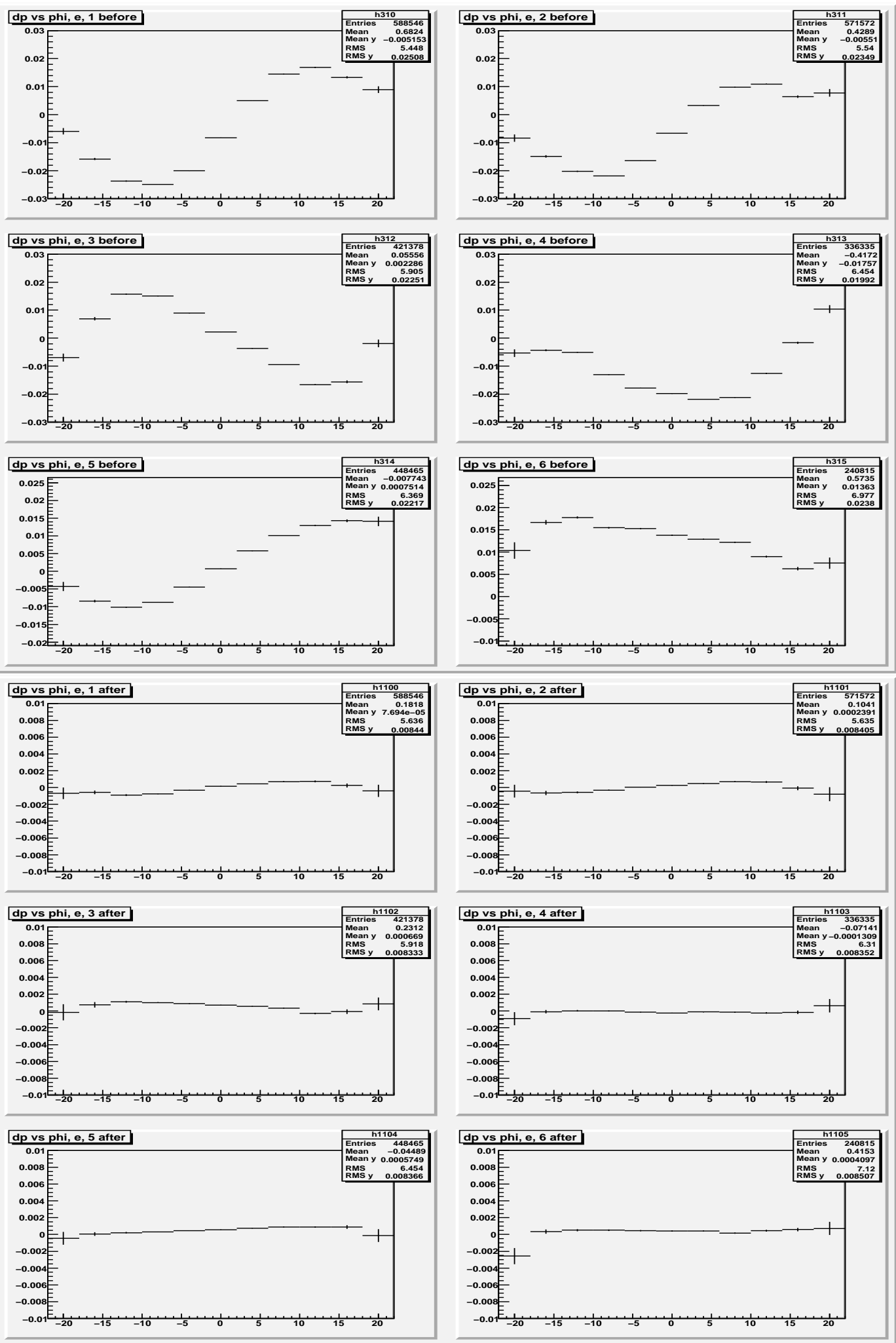

Figure 4.3: Electron hbprof distributions of $d p$ vs. $\phi_{e}$ for all sectors before (top) and after (bottom) corrections. Note the different $d p$ axis scales in the before and after plots. 


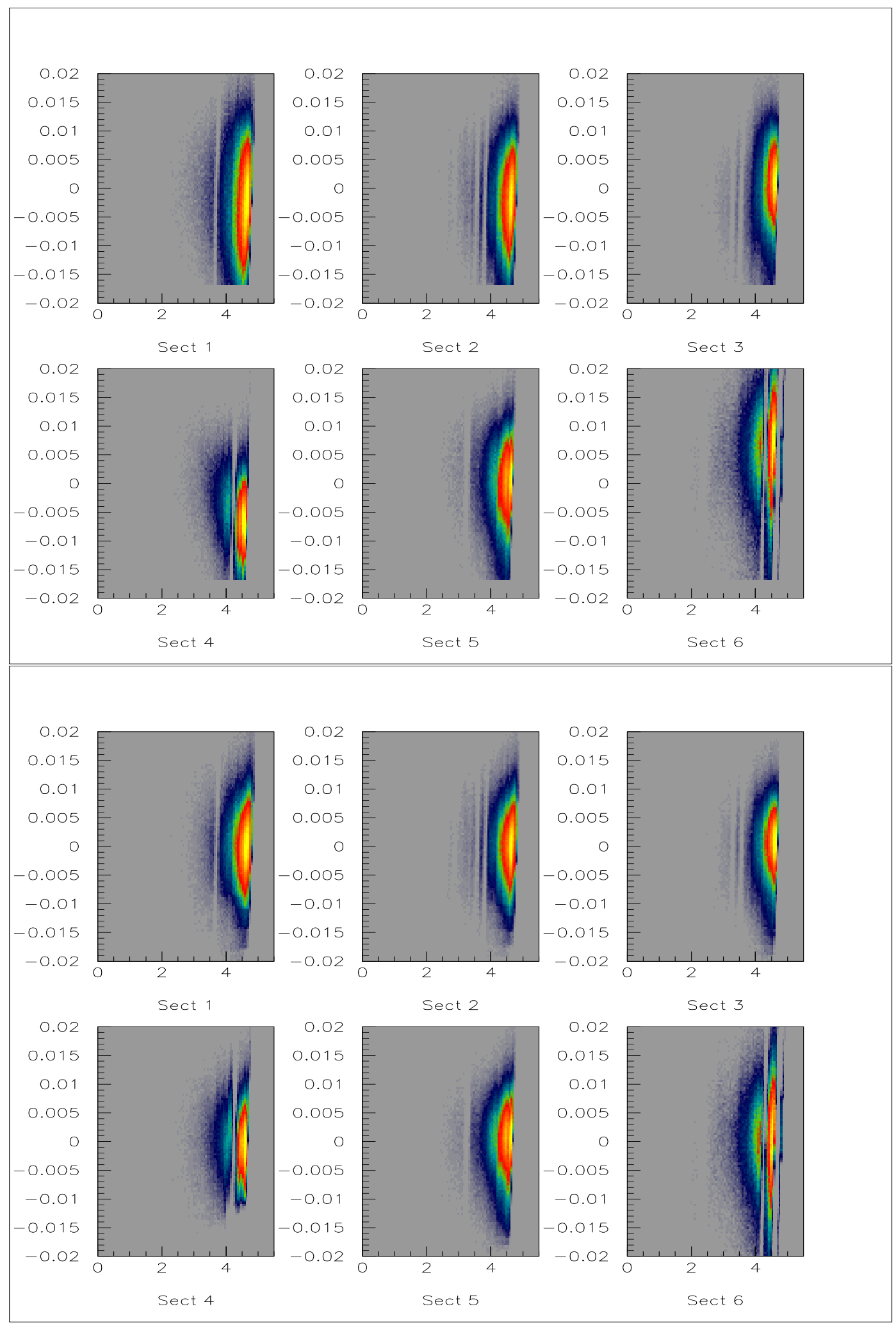

Figure 4.4: Electron $d p / p$ vs. $p_{e}$ distributions before (top) and after (bottom) corrections. The distributions are centered at zero after applying the momentum corrections. The gaps in these plots are the result of the removed SC paddles. 


\section{FIU Hadron Corrections}

Hadron momentum correction are performed after the electron corrections are finalized and applied. All three reactions, namely $e p \rightarrow e p$ elastic scattering, $e p \rightarrow e^{\prime} \pi^{+} n$ and $e p \rightarrow e^{\prime} K^{+} \Lambda$ reactions are used for this purpose. Analysis showed that one can combine and generate the single correction vectors for all positively charged hadrons. The elastic channel selection is described in Section 4.3. Inelastic channels are selected by applying the missing mass cut, requiring it to be around the $n$ and $\Lambda$ mass, respectively. A $W>1.025 \mathrm{GeV}$ cut is also applied to remove the elastic events from the inelastic channels. The hadron momenta are derived from 4-momentum conservation.

Here again the two dimensional histograms of $d p=p_{h}^{\text {meas }}-p_{h}^{\text {calc }}$ vs. $\theta_{h}$ and $\phi_{h}$ are created. These two dimensional histograms are converted into profiles by using the PAW hbprof command. The profile histograms are then read into the vhth and vhph vectors and are applied as corrections.

$$
p_{h}^{\text {corrected }}=p_{h}^{\text {meas }}-\operatorname{vhph}\left(\operatorname{bin}_{\phi}\right)-\operatorname{vhth}\left(\operatorname{bin}_{\theta}\right),
$$

where the vhth and vhph vectors are defined for each sector separately to remove the $\theta_{h}$ and $\phi_{h}$ dependencies, respectively, of $d p$. Only two iterations are performed for the hadron momentum corrections. The binning of the hadron kinematic variables is shown in Table 4.4 .

\begin{tabular}{|r|r|r|r|}
\hline Variable & Range & Width & Number of Bins \\
\hline$\theta_{h}$ & $8^{\circ} \rightarrow 92^{\circ}$ & $4^{\circ}$ & 21 \\
\hline$\phi_{h}$ & $-26^{\circ} \rightarrow 26^{\circ}$ & $4^{\circ}$ & 13 \\
\hline
\end{tabular}

Table 4.4: Binning of the hadron $\theta_{h}$ and $\phi_{h}$ variables.

Fig. 4.5 shows the fractional momentum correction vs. $p_{h}$. The sharp transition in Fig. 4.5 is because of the elastic protons. The momentum corrections are on the order of $1.5 \%$ for hadrons. Although a full set of momentum corrections for hadrons 


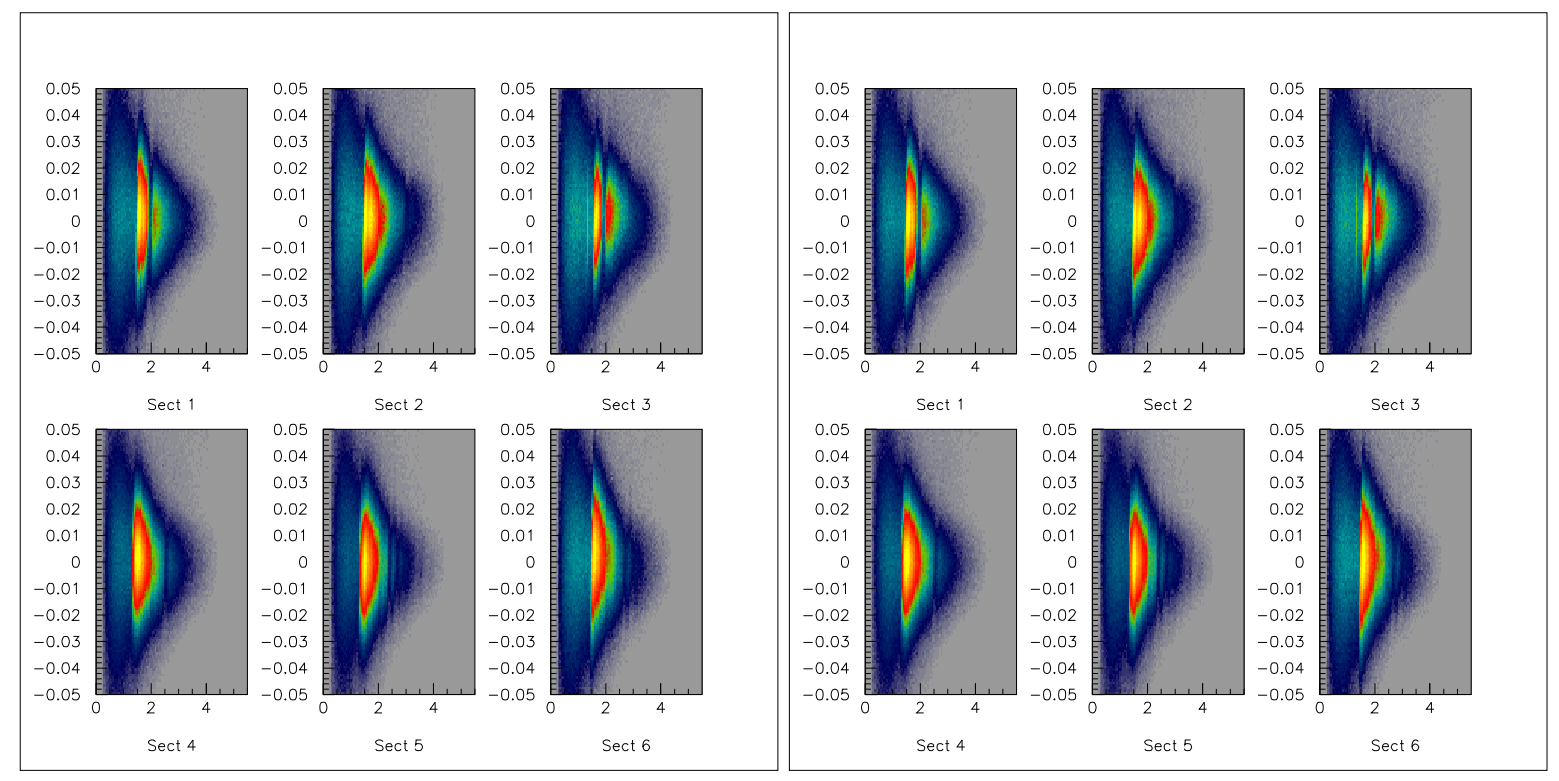

Figure 4.5: Hadron $d p / p$ vs. $p_{h}$ distributions before (left) and after (right) the hadron momentum corrections. The sharp transitions in the se plots are the result of the elastic events. The gaps are the result of the removed SC paddles.

were developed, they were not used in the final analysis since they did not improve and occasionally even worsen the resolution.

\section{Cross Check}

As a cross check, the momentum corrections from FIU and INFN are tested on $e p \rightarrow$ $e^{\prime} K^{+} \Lambda$ reaction. The hyperon missing mass spectra are calculated for each sector, $\theta_{e}$, and $W$ bin using both momentum correction methods. The summaries of the obtained centroids and widths using both corrections are presented in Table 4.5, for each sector, and in Table 4.6, for each $\theta_{e}$ bin. In both tables $e^{-}$(FIU) refers to the case when only FIU $e^{-}$momentum corrections are applied, Hadron (FIU) refers to the case when both electron and hadron corrections are applied. Hadron (INFN) refers to the case when both electron and hadron momentum corrections calculated by the INFN method are applied. The results show that although there is an improvement at small electron angles, at large angles the widths got worse after applying the momentum corrections.

Table 4.7 summarizes the centroids and the widths before and after each set of cor- 


\begin{tabular}{|r|r|r|r|r|r|r|r|r|}
\hline \multicolumn{10}{|c|}{$M M\left(e^{\prime} K^{+}\right)$CENTROIDS AND WIDTHS } \\
\hline Sector & $\begin{array}{r}\text { NO corr } \\
{[\mathrm{MeV}]}\end{array}$ & $\begin{array}{r}\text { NO corr } \sigma \\
{[\mathrm{MeV}]}\end{array}$ & $\begin{array}{r}e^{-} \\
(\mathrm{FIU})\end{array}$ & $\sigma_{F I U}$ & $\begin{array}{r}\text { Hadron } \\
(\text { FIU) }\end{array}$ & $\sigma_{F I U}$ & $\begin{array}{r}\text { Hadron } \\
(\text { INFN) }\end{array}$ & $\sigma_{I N F N}$ \\
\hline S1 & 1118 & 18.5 & 1115 & 18.3 & 1114 & 17.7 & 1116 & 18.3 \\
\hline S2 & 1118 & 16.8 & 1114 & 17.2 & 1113 & 16.7 & 1117 & 17.0 \\
\hline S3 & 1116 & 16.9 & 1116 & 16.6 & 1114 & 16.0 & 1116 & 16.7 \\
\hline S4 & 1120 & 16.7 & 1112 & 16.6 & 1113 & 16.3 & 1118 & 16.5 \\
\hline S5 & 1114 & 16.9 & 1115 & 16.4 & 1116 & 16.1 & 1115 & 16.9 \\
\hline S6 & 1107 & 21.1 & 1119 & 18.6 & 1120 & 18.4 & 1111 & 21.2 \\
\hline
\end{tabular}

Table 4.5: Hyperon missing mass distributions for each sector before and after momentum corrections. Summary of centroids and widths after each set of corrections.

\begin{tabular}{|r|r|r|r|r|r|r|r|r|}
\hline \multicolumn{10}{|c|}{$M M\left(e^{\prime} K^{+}\right)$CENTROIDS AND WIDTS } \\
\hline$\theta_{e}$ & $\begin{array}{r}\text { NO corr } \\
{[\mathrm{MeV}]}\end{array}$ & $\begin{array}{r}\text { NO corr } \sigma \\
{[\mathrm{MeV}]}\end{array}$ & $\begin{array}{r}e^{-} \\
(\text {FIU) }\end{array}$ & $\sigma_{F I U} I$ & $\begin{array}{r}\text { Hadron } \\
(\text { FIU) }\end{array}$ & $\sigma_{F I U}$ & $\begin{array}{r}\text { Hadron } \\
(\text { INFN) }\end{array}$ & $\sigma_{I N F N}$ \\
\hline $12^{\circ} \rightarrow 16^{\circ}$ & 1116 & 17.0 & 1114 & 15.1 & 1114 & 14.9 & 1115 & 17.2 \\
\hline $16^{\circ} \rightarrow 20^{\circ}$ & 1116 & 17.6 & 1115 & 16.3 & 1114 & 16.0 & 1116 & 17.5 \\
\hline $20^{\circ} \rightarrow 24^{\circ}$ & 1116 & 18.2 & 1115 & 17.3 & 1115 & 17.0 & 1115 & 18.2 \\
\hline $24^{\circ} \rightarrow 28^{\circ}$ & 1116 & 19.0 & 1116 & 19.0 & 1116 & 18.6 & 1115 & 18.8 \\
\hline $28^{\circ} \rightarrow 32^{\circ}$ & 1115 & 19.5 & 1116 & 21.3 & 1116 & 20.2 & 1115 & 19.8 \\
\hline $32^{\circ} \rightarrow 36^{\circ}$ & 1114 & 20.6 & 1117 & 21.5 & 1116 & 21.0 & 1115 & 20.4 \\
\hline $36^{\circ} \rightarrow 40^{\circ}$ & 1115 & 21.0 & 1116 & 23.2 & 1116 & 23.0 & 1115 & 21.3 \\
\hline
\end{tabular}

Table 4.6: Hyperon missing mass distributions for each $\theta_{e}$ bin. Summary of centroids and widths after each set of corrections. 


\section{$M M\left(e^{\prime} K^{+}\right)$CENTROIDS AND WIDTHS}

\begin{tabular}{|r|r|r|r|r|r|r|r|r|}
\hline $\begin{array}{r}W \text { Bin } \\
{[\mathrm{GeV}]}\end{array}$ & $\begin{array}{r}\text { NO corr } \\
{[\mathrm{MeV}]}\end{array}$ & $\begin{array}{r}\text { NO corr } \sigma \\
{[\mathrm{MeV}]}\end{array}$ & $\begin{array}{r}e^{-} \\
(\mathrm{FIU})\end{array}$ & $\sigma_{F I U}$ & $\begin{array}{r}\text { Hadron } \\
(\text { FIU) }\end{array}$ & $\sigma_{F I U}$ & $\begin{array}{r}\text { Hadron } \\
(\mathrm{INFN})\end{array}$ & $\sigma_{I N F N}$ \\
\hline $1.6-1.65$ & 1103 & 15.3 & 1105 & 14.5 & 1105 & 15.6 & 1104 & 15.2 \\
\hline $1.65-1.7$ & 1112 & 17.1 & 1111 & 14.8 & 1111 & 15.6 & 1112 & 16.1 \\
\hline $1.7-1.75$ & 1115 & 17.4 & 1114 & 15.6 & 1114 & 16.4 & 1115 & 16.5 \\
\hline $1.75-1.8$ & 1116 & 17.2 & 1115 & 16.0 & 1115 & 16.9 & 1116 & 16.2 \\
\hline $1.8-1.85$ & 1116 & 18.0 & 1115 & 16.5 & 1115 & 17.5 & 1116 & 16.5 \\
\hline $1.85-1.9$ & 1117 & 17.9 & 1116 & 17.2 & 1115 & 18.4 & 1116 & 16.4 \\
\hline $1.9-1.95$ & 1116 & 18.5 & 1116 & 17.9 & 1115 & 18.8 & 1116 & 16.9 \\
\hline $1.95-2.0$ & 1117 & 19.1 & 1116 & 18.5 & 1115 & 19.8 & 1116 & 17.0 \\
\hline $2.0-2.05$ & 1116 & 19.5 & 1115 & 19.3 & 1115 & 20.7 & 1116 & 17.6 \\
\hline $2.05-2.1$ & 1116 & 19.1 & 1116 & 19.4 & 1115 & 20.6 & 1116 & 17.1 \\
\hline $2.1-2.15$ & 1116 & 19.7 & 1116 & 20.3 & 1115 & 21.4 & 1116 & 17.4 \\
\hline $2.15-2.2$ & 1117 & 20.2 & 1116 & 20.9 & 1116 & 22.0 & 1117 & 17.8 \\
\hline $2.2-2.25$ & 1116 & 20.6 & 1116 & 21.1 & 1115 & 22.2 & 1116 & 18.6 \\
\hline $2.25-2.3$ & 1117 & 22.4 & 1116 & 22.9 & 1116 & 24.2 & 1116 & 19.6 \\
\hline $2.3-2.35$ & 1117 & 22.4 & 1116 & 23.3 & 1116 & 24.5 & 1116 & 19.6 \\
\hline $2.35-2.4$ & 1116 & 22.5 & 1116 & 23.4 & 1116 & 24.5 & 1116 & 19.3 \\
\hline $2.4-2.45$ & 1116 & 23.8 & 1116 & 24.6 & 1115 & 25.7 & 1115 & 20.2 \\
\hline $2.45-2.5$ & 1116 & 25.0 & 1117 & 25.0 & 1116 & 26.6 & 1115 & 20.6 \\
\hline $2.5-2.55$ & 1116 & 24.9 & 1116 & 26.8 & 1116 & 28.2 & 1115 & 22.0 \\
\hline $2.55-2.6$ & 1116 & 25.7 & 1116 & 27.2 & 1115 & 28.8 & 1115 & 23.2 \\
\hline $2.6-2.65$ & 1117 & 28.4 & 1118 & 29.9 & 1117 & 30.9 & 1116 & 26.3 \\
\hline $2.65-2.7$ & 1117 & 28.2 & 1118 & 28.5 & 1118 & 29.6 & 1116 & 23.6 \\
\hline $2.7-2.75$ & 1118 & 28.7 & 1118 & 28.8 & 1118 & 31.3 & 1116 & 26.0 \\
\hline $2.75-2.8$ & 1118 & 31.5 & 1119 & 29.7 & 1117 & 29.5 & 1117 & 27.4 \\
\hline
\end{tabular}

Table 4.7: $\Lambda$ missing mass distributions for each $W$ bin. Summary of centroids and widths after each set of corrections. 
rections for each $W$ bin. The results show that at small values of $W$, the FIU corrections work a little better, but at large $W$ the INFN corrections do a better job. As a conclusion, we decided to use the electron momentum corrections developed by the FIU group. No hadron momentum corrections are applied in the final analysis.

\subsection{Background Subtraction}

Despite all the cuts applied during the final state selection and the particle identification, it is impossible to completely eliminate the pion background and the $\Sigma^{0}$ contributions in the $\Lambda$ missing mass spectra. This is partly because of the low magnetic field used during the data acquisition, which leads to a worse resolution compared to larger magnetic fields. In order to obtain clean $\Lambda$ samples, the missing mass spectra in each kinematic bin are fitted by a 16-parameter function as described below. For this purpose the $\Lambda$

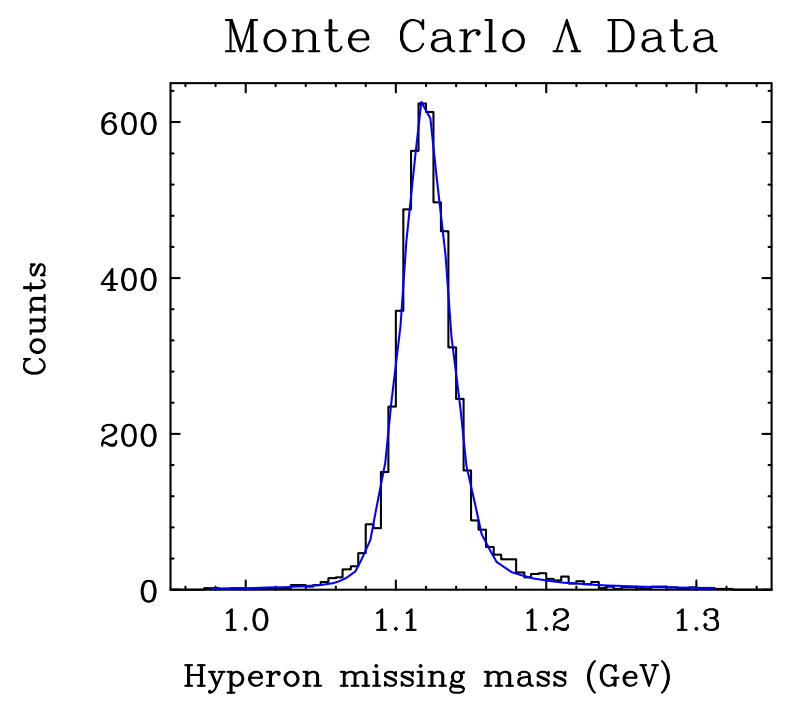

Figure 4.6: Sample fit of a $\Lambda$ MC template. A Gaussian plus an asymmetrical Lorentzian on both sides of the Gaussian centroid was fit to the $\Lambda$ peak.

missing mass spectra were generated with an extended pion missing-mass-squared cut (up to $0.07 \mathrm{GeV}^{2}$ ) to include all $\Sigma^{0} s$ (Fig. 3.16 a)). Expanding pion missing-masssquared cut is necessary for the proper fitting of the $\Sigma^{0}$ tail underneath the $\Lambda$ peak. The lineshapes of the $\Lambda$ and $\Sigma^{0}$ peaks are motivated by Monte Carlo simulation templates. 
Fig. 4.6 shows a sample fit to a $\Lambda$ template. The $\Lambda$ peak is fitted by a Gaussian plus an asymmetrical Lorentzian on both sides of the Gaussian centroid. It is necessary to use an asymmetrical Lorentzian in order to take into account the resolution effects on the low-mass side and the radiative effects on the high-mass side of the peak.

As part of the background studies for this analysis, pions and protons were intentionally misidentified as kaons then treated as normal kaon events. The $\Delta t$ vs. $p$ distributions for intentionally misidentified pions and protons are overlayed on the same distributions for kaons as shown in Fig. 4.7. From this plot one can see that the pions can be misidentified as kaons starting at about $1.5 \mathrm{GeV}$ and the protons at about 3.0 $\mathrm{GeV}$. Since most of the kaons have momentum less than $3.0 \mathrm{GeV}$, the main source of the background is pion misidentification. The pion background shape strongly depends on

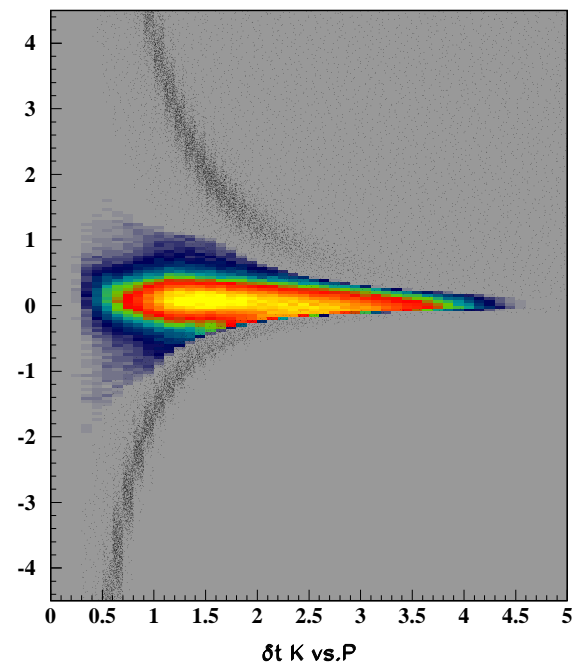

Figure 4.7: $\Delta t$ vs. $p$ distributions for kaons with misidentified pion and proton bands overlayed.

the kinematic bins, as can be seen from Fig. 4.8. The changing shape did not allow any functional form to be used for background fitting throughout the full kinematic range. Instead, the pion background templates are generated from the data, by intentionally misidentifying the pions as kaons. The background templates are smoothed in order to get rid of the statistical fluctuations as shown in Fig. 4.9. These smoothed histograms are then used for background subtraction. 

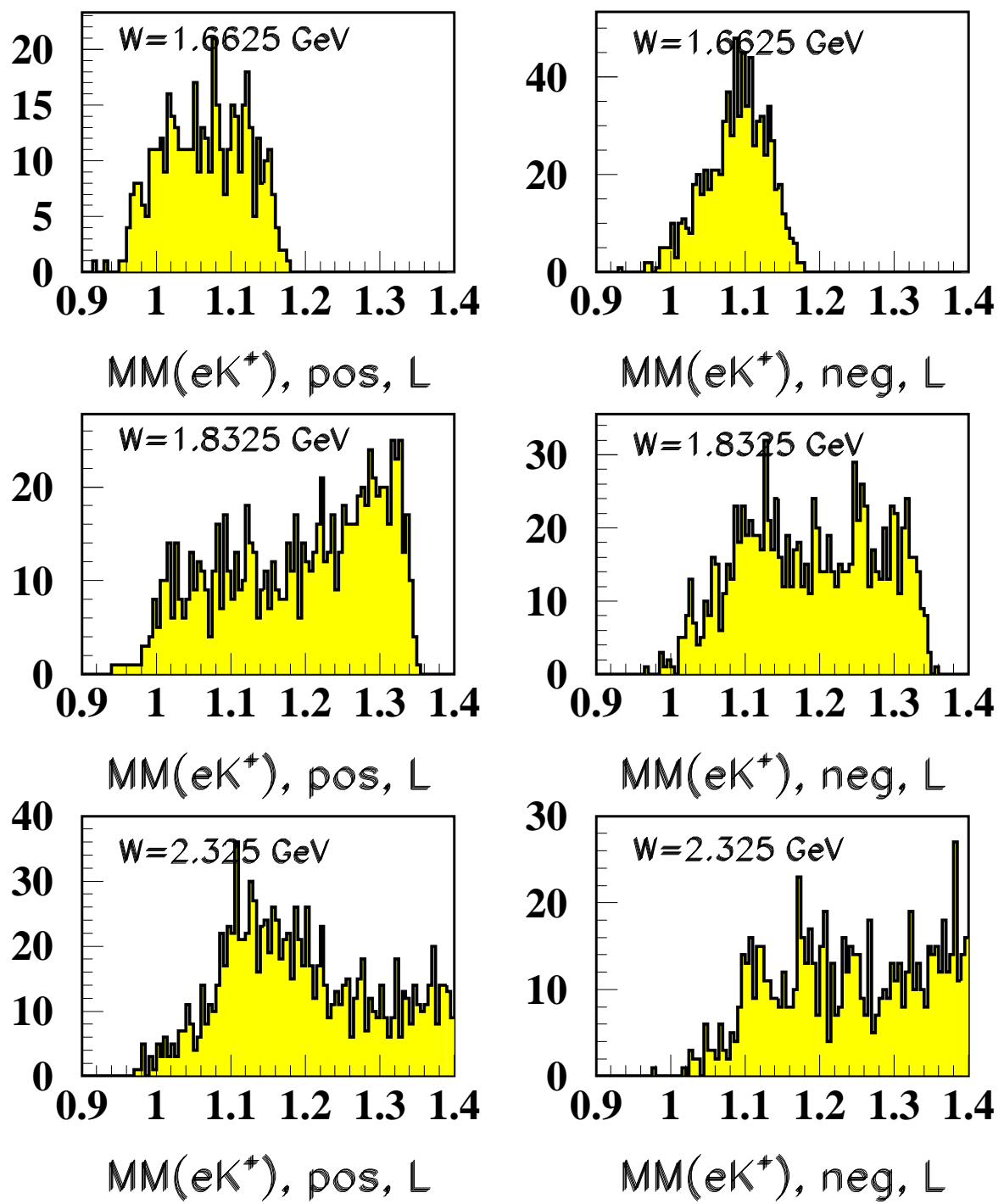

Figure 4.8: Pion background templates for different $W$ bins for $0.8<$ $\cos \theta_{K}^{C M}<1$. Plots show the distributions for $p$ 's moving both along and opposite to the longitudinal quantization axis. 


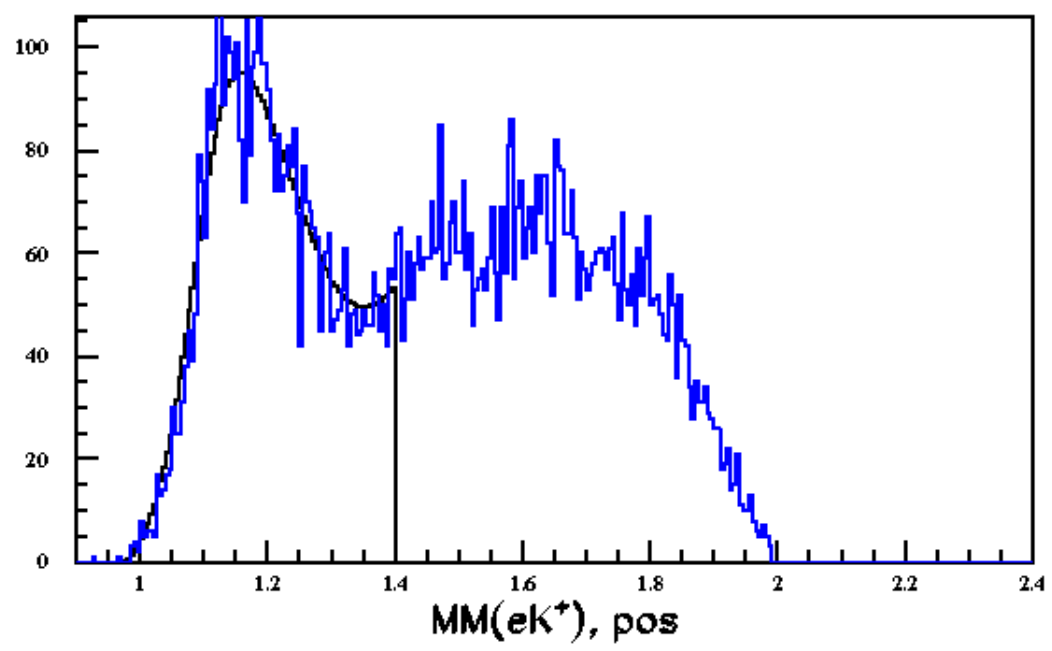

Figure 4.9: Pion background template for $p$ 's moving along the normal quantization axis. The black curve is the smoothed template used for background subtraction.

\section{Fit Function}

Since the centroids and the widths of the $\Lambda$ and $\Sigma^{0}$ peaks do not depend on $p$ angles, the hyperon missing mass histograms for $p$ 's moving along (forward) and opposite (backward) to the given quantization axes are fit simultaneously for each kinematic bin. In PAW, in order to accomplish simultaneous fit of two histograms, one of the histograms is shifted by a constant amount with respect to the other and put into a single histogram. In this analysis the histogram corresponding to backward $p$ angles $\left(\cos \theta_{p}^{R F}<0\right)$ is shifted by $1.0 \mathrm{GeV}$. In this case the centroids of the $\Lambda$ and $\Sigma^{0}$ peaks in the fit function for the second histogram must be shifted by the same constant amount.

$$
\begin{gathered}
\mu_{\Lambda} \rightarrow \mu_{\Lambda}+\text { shift } \\
\mu_{\Sigma} \rightarrow \mu_{\Sigma}+\text { shift. }
\end{gathered}
$$

To avoid any overlaps between the two histograms, due to the higher mass hyperon contributions, all bins beyond $1.4 \mathrm{GeV}$ are set to zero for the forward angle histograms. For the backward angle histograms, all bins below $1.9 \mathrm{GeV}$ are set to zero. The same is done for the respective pion background templates. 
The hyperon missing mass spectra in each kinematic bin are fit by a 16-parameter function with parameters defined as follows:

$a_{1}$ is the $\Lambda$ Gaussian amplitude,

$a_{2}$ is the $\Lambda$ Gaussian centroid, $\left(a_{2}=\mu_{\Lambda}\right)$

$a_{3}$ is the $\Lambda$ Gaussian width,

$a_{4}$ is the $\Lambda$ Lorentzian relative amplitude,

$a_{5}$ is the $\Lambda$ left Lorentzian width,

$a_{6}$ is the $\Lambda$ right Lorentzian width,

$a_{7}$ is the $\Lambda$ Gaussian amplitude for the second histogram,

$a_{8}$ is the $\Sigma^{0}$ Gaussian amplitude,

$a_{9}$ is the $\Sigma^{0}$ Gaussian centroid, $\left(a_{8}=\mu_{\Sigma}\right)$

$a_{10}$ is the $\Sigma^{0}$ Gaussian width,

$a_{11}$ is the $\Sigma^{0}$ Lorentzian relative amplitude,

$a_{12}$ is the $\Sigma^{0}$ left Lorentzian width,

$a_{13}$ is the $\Sigma^{0}$ second Lorentzian width,

$a_{14}$ is the $\Sigma^{0}$ Gaussian amplitude for the second histogram,

$a_{15}$ is the background amplitude.

$a_{16}$ is the background amplitude for the second histogram.

Two constraints are provided by the fact that the ratios of the Gaussian and the Lorentzian amplitudes must be the same for the forward and backward $p$ angles. These constraints allow elimination of the corresponding relative amplitudes as free parameters. Only the absolute amplitudes are allowed to vary freely.

For the fit procedure the full missing mass range is divided into three sections: a) below the $\Lambda$ Gaussian centroid, b) between the $\Lambda$ and $\Sigma^{0}$ Gaussian centroids and c) above the $\Sigma^{0}$ Gaussian centroid. The $\Lambda$ and $\Sigma^{0}$ peaks are fitted by a Gaussian plus an asymmetrical Lorentzian in each side of the centroid. 
1. For $\cos \theta_{p}^{R F}>0$.

a) Left side of the $\Lambda$ centroid $\left(x \leq \mu_{\Lambda}\right)$

$$
y_{1}=a_{1}\left(G_{\Lambda^{+}}+a_{4} \cdot L_{\Lambda^{1}}\right)+a_{8}\left(G_{\Sigma^{+}}+a_{11} \cdot L_{\Sigma^{1}}\right)+b g_{1},
$$

where

$$
\begin{aligned}
G_{\Lambda^{+}} & =\exp \left(-0.5\left(\frac{x-a_{2}}{a_{3}}\right)^{2}\right) \\
L_{\Lambda^{1}} & =\frac{1}{a_{5}^{2}+\left(x-a_{2}^{2}\right)} \\
G_{\Sigma^{+}} & =\exp \left(-0.5\left(\frac{x-a_{9}}{a_{10}}\right)^{2}\right) \\
L_{\Sigma^{1}} & =\frac{1}{a_{12}^{2}+\left(x-a_{9}^{2}\right)} \\
b g_{1} & =a_{15} \cdot\left[\text { Template }_{1}\right] .
\end{aligned}
$$

b) Between the $\Lambda$ and $\Sigma^{0}$ centroids $\left(\mu_{\Lambda}<x \leq \mu_{\Sigma}\right)$

$$
y_{2}=a_{1}\left(G_{\Lambda^{+}}+N_{l} \cdot L_{\Lambda^{2}}\right)+a_{8}\left(G_{\Sigma^{+}}+a_{11} \cdot L_{\Sigma^{1}}\right)+b g_{1},
$$

where $G_{\Lambda^{+}}, G_{\Sigma^{+}}, L_{\Sigma^{1}}$ and $b g_{1}$ are defined as before and

$$
L_{\Lambda^{2}}=\frac{1}{a_{6}^{2}+\left(x-a_{2}\right)^{2}} .
$$

At the $\Lambda$ centroid, $x=\mu_{\Lambda}, y_{1}=y_{2}$ so that $a_{4} L_{\Lambda^{1}}=N_{l} L_{\Lambda^{2}}$. This allows one to express the $N_{l}$ amplitude in terms of the other parameters:

$$
N_{l}=\frac{a_{6}^{2} a_{4}}{a_{5}^{2}}
$$

c) Right side of the $\Sigma^{0}$ centroid $\left(x>\mu_{\Sigma}\right)$

$$
y_{3}=a_{1}\left(G_{\Lambda^{+}}+N_{l} \cdot L_{\Lambda^{2}}\right)+a_{8}\left(G_{\Sigma^{+}}+N_{s} \cdot L_{\Sigma^{2}}\right)+b g_{1},
$$


where $G_{\Lambda^{+}}, G_{\Sigma^{+}}, L_{\Lambda^{2}}$ and $b g_{1}$ are defined as before and

$$
L_{\Sigma^{2}}=\frac{1}{a_{13}^{2}+\left(x-a_{9}\right)^{2}} .
$$

Again, at $x=\mu_{\Sigma}, \Sigma^{0}$ centroid, $a_{11} L_{\Sigma^{1}}=N_{s} L_{\Sigma^{2}}$, so the $N_{s}$ amplitude can be expressed in terms of the other parameters as:

$$
N_{s}=\frac{a_{13}^{2} a_{11}}{a_{12}^{2}}
$$

Finally, to fit one $\Lambda$ missing mass histogram, the fitting function $F_{1}(x)$ is defined as:

$$
F_{1}(x)= \begin{cases}y_{1}, & \left(x \leq \mu_{\Lambda}\right) \\ y_{2}, & \left(\mu_{\Lambda}<x \leq \mu_{\Sigma}\right) \\ y_{3}, & \left(x>\mu_{\Sigma}\right),\end{cases}
$$

where $\mu_{\Lambda}=a_{2}$ and $\mu_{\Sigma}=a_{9}$ denote the $\Lambda$ and $\Sigma^{0}$ Gaussian centroids.

2. For $\cos \theta_{p}^{R F}<0$.

a) Left side of the $\Lambda$ centroid $\left(x \leq \mu_{\Lambda}\right)$

$$
y_{4}=a_{7}\left(G_{\Lambda^{-}}+a_{4} \cdot L_{\Lambda^{3}}\right)+a_{14}\left(G_{\Sigma^{-}}+a_{11} \cdot L_{\Sigma^{3}}\right)+b g_{2},
$$

where

$$
\begin{aligned}
G_{\Lambda^{-}} & =\exp \left(-0.5\left(\frac{x-\left(a_{2}+1\right)}{a_{3}}\right)^{2}\right) \\
L_{\Lambda^{3}} & =\frac{1}{a_{5}^{2}+\left(x-\left(a_{2}+1\right)\right)^{2}} \\
G_{\Sigma^{-}} & =\exp \left(-0.5\left(\frac{x-\left(a_{9}+1\right)}{a_{10}}\right)^{2}\right) \\
L_{\Sigma^{3}} & =\frac{1}{a_{12}^{2}+\left(x-\left(a_{9}+1\right)\right)^{2}} \\
b g_{2} & =a_{16} \cdot\left[\text { Template }_{2}\right] .
\end{aligned}
$$


b) Between the $\Lambda$ and $\Sigma^{0}$ centroids $\left(\mu_{\Lambda}<x \leq \mu_{\Sigma}\right)$

$$
y_{5}=a_{7}\left(G_{\Lambda^{-}}+N_{l} \cdot L_{\Lambda^{4}}\right)+a_{14}\left(G_{\Sigma^{-}}+a_{11} \cdot L_{\Sigma^{3}}\right)+b g_{2},
$$

where $G_{\Lambda^{-}}, G_{\Sigma^{-}}, L_{\Sigma^{3}}$ and $b g_{2}$ are defined as before and

$$
L_{\Lambda^{4}}=\frac{1}{a_{6}^{2}+\left(x-\left(a_{2}+1\right)\right)^{2}} .
$$

c) Right side of the $\Sigma^{0}$ centroid $\left(x>\mu_{\Sigma}\right)$

$$
y_{6}=a_{7}\left(G_{\Lambda^{-}}+N_{l} \cdot L_{\Lambda^{4}}+a_{14}\left(G_{\Sigma^{-}}+N_{s} \cdot L_{\Sigma^{4}}\right)+b g_{2},\right.
$$

where $G_{\Lambda^{-}}, G_{\Sigma^{-}}, L_{\Lambda^{4}}$ and $b g_{2}$ are defined as before and

$$
L_{\Sigma^{4}}=\frac{1}{a_{13}^{2}+\left(x-\left(a_{9}+1\right)\right)^{2}} .
$$

The fit function for the second histogram is defined as:

$$
F_{2}(x)=\left\{\begin{array}{l}
y_{4},\left(x \leq \mu_{\Lambda}+1\right) \\
y_{5},\left(\mu_{\Lambda}+1<x \leq \mu_{\Sigma}+1\right) \\
y_{6},\left(x>\mu_{\Sigma}+1\right)
\end{array}\right.
$$

Note the shift of the Gaussian and Lorentzian centroids by $1 \mathrm{GeV}$ in the fit function of the second histogram.

The total 16-parameter fit function used for the analysis is defined as:

$$
F(x)=\left\{\begin{array}{l}
F_{1},(0.9 \leq x \leq 1.4) \\
0,(1.4 \leq x \leq 1.9) \\
F_{2},(1.9 \leq x \leq 2.4)
\end{array}\right.
$$


A typical sample fits are shown in Fig. 4.10. In order to have meaningful fits, it is necessary to reduce the number of free parameters. The reduction of free parameters is done by parameterizing the shape parameters as a function of $W$. This parameterization insures that the fit parameters vary smoothly from one kinematic bin to another. The final parameterization used for this analysis is given in Appendix C. The final results are obtained by six-parameter fits, where only the absolute amplitudes are allowed to vary freely. The reduced $\chi^{2}$ distributions, shown in Fig. 4.11 for two $\cos \theta_{K}^{C M}$ bins, demonstrate that very reasonable fits are achieved.

After the fitting procedure is complete, the $\Lambda, \Sigma^{0}$ and the background contributions to the total peak can be cleanly separated. The number of $\Lambda$ 's in each kinematic bin, corresponding to forward and backward $p$ angles, are determined by integrating the functions corresponding to the $\Lambda$ peak within the missing mass range from $1.05 \mathrm{GeV}$ to 1.15 GeV (forward $p$ angles) and from $2.05 \mathrm{GeV}$ to $2.15 \mathrm{GeV}$ (backward $p$ angles). The obtained yields, $N_{ \pm}$, are now background subtracted and can be used with acceptance corrections as described in Section 4.5. The statistical uncertainties of the uncorrected yields $N_{ \pm}$are determined by:

$$
d N_{ \pm}^{2}=\sum_{i}^{n} \sum_{j}^{n} \frac{\partial f_{\Lambda}}{\partial a_{i}} \frac{\partial f_{\Lambda}}{\partial a_{j}} \epsilon_{i j}
$$

where $n$ is the number of free parameters, $\epsilon_{i j}$ is the correlation matrix of the parameters. The correlation matrix, $\epsilon_{i j}$, reflects the fact that the fit parameters are not completely independent. $f_{\Lambda}$ is the $\Lambda$ peak fit function integrated within the missing mass range from $1.05 \mathrm{GeV}$ to $1.15 \mathrm{GeV}\left(d N_{+}\right)$or $2.05 \mathrm{GeV}$ to $2.15 \mathrm{GeV}\left(d N_{-}\right)$.

\subsection{Acceptance Corrections}

In order to obtain reliable results it is necessary to calculate the acceptance-corrected yields for each kinematic bin. A full scale GEANT Monte Carlo simulation was performed to correct the experimental yields for the acceptance. In the first stage of the 

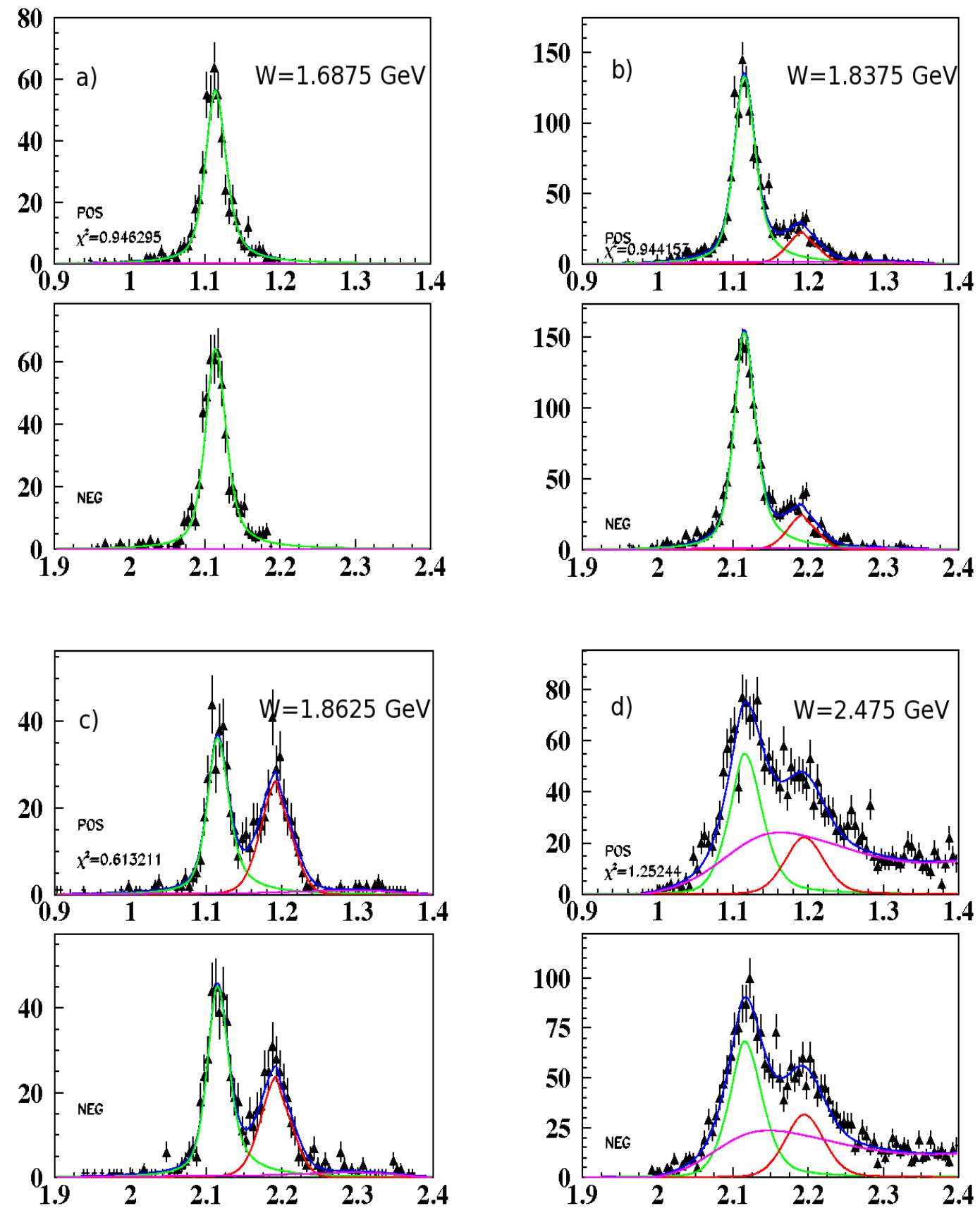

Figure 4.10: Typical fits to $\Lambda$ missing mass histograms at different $W$ bins using Eq. 4.9. The histograms corresponding to forward and backward $p$ angles are fitted simultaneously. Note that the backward $p$ histograms are shifted by $1 \mathrm{GeV}$. The green curve corresponds to the $\Lambda$ peak, the red curve corresponds to $\Sigma^{0}$ peak, the purple curve is the background and the blue curve is the total fit function. 

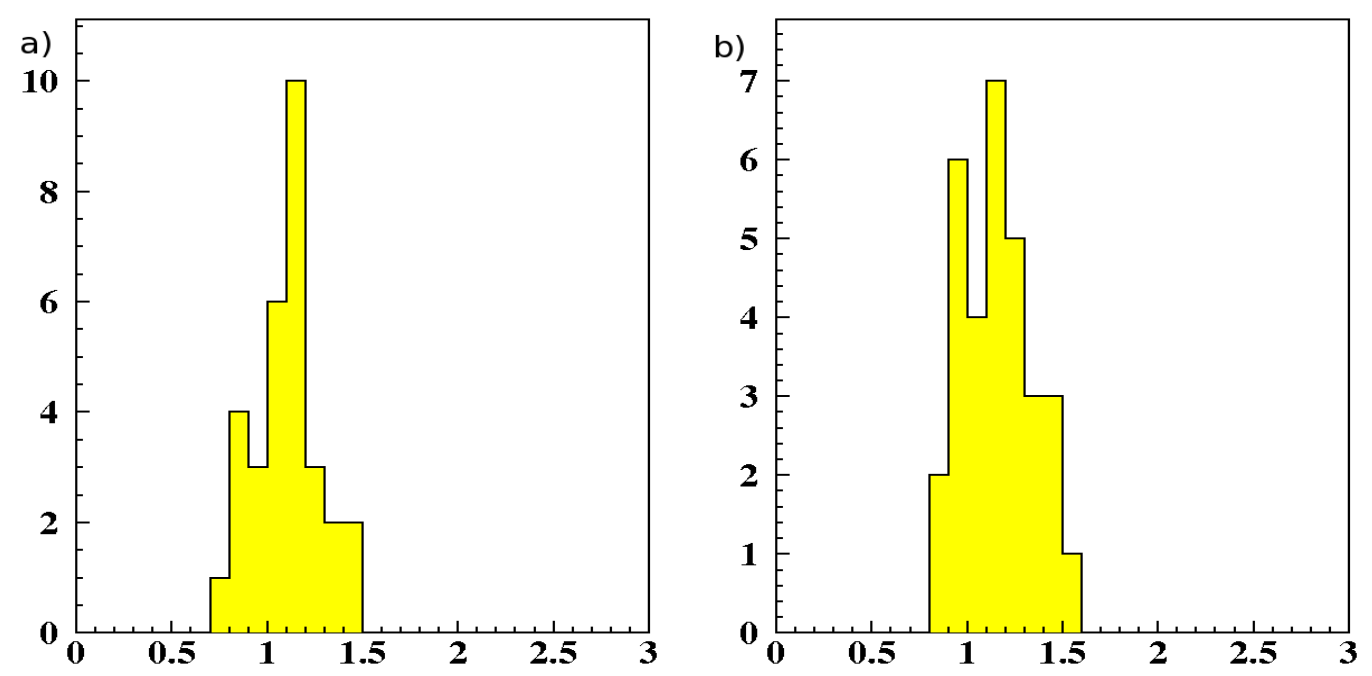

Figure 4.11: Reduced $\chi^{2}$ distributions for a) $0.6<\cos \theta_{K}^{C M}<0.8$ and b) $0.8<$ $\cos \theta_{K}^{C M}<1$ bins.

simulation the $e p \rightarrow e^{\prime} K^{+} \Lambda$ reaction with $\Lambda \rightarrow p \pi^{-}$decay events are generated by the FSGEN phase space generator with a modified $t$-slope. The generator scales the phase space cross section by a factor of $e^{-b t}$, where $b$ is the $t$-slope parameter $\left(b=0.3 \mathrm{GeV}^{-2}\right.$, $t=t_{\text {Mandelstam }}$ ), to yield the probability distribution that better matches the data. The larger the $b$, the more kaons are forced to go at forward direction, generating $t$-channel production events. The radiative effects are partially taken into account in the generator. They only include the final state radiation on the final scattered electrons. In the second step, the generated events are processed with the GSIM package, which is the GEANT simulation of the CLAS detector. The particles from the generated events are propagated through the CLAS and the detector response is recorded much the same way as for the experimental data. The GSIM simulation of the CLAS assumes a perfect detector system, so the inefficiencies and the resolutions of the different detector components are not properly simulated. These are taken into account in the next step by the GSIM post-processing (GPP) package, which allows the user to smear the DC and TOF times by factors to better match the experimental data. In the final stage, the simulated data are cooked using the same version of the analysis executable used for the cooking of the actual data. After the final reconstruction, the data are analyzed by the same 


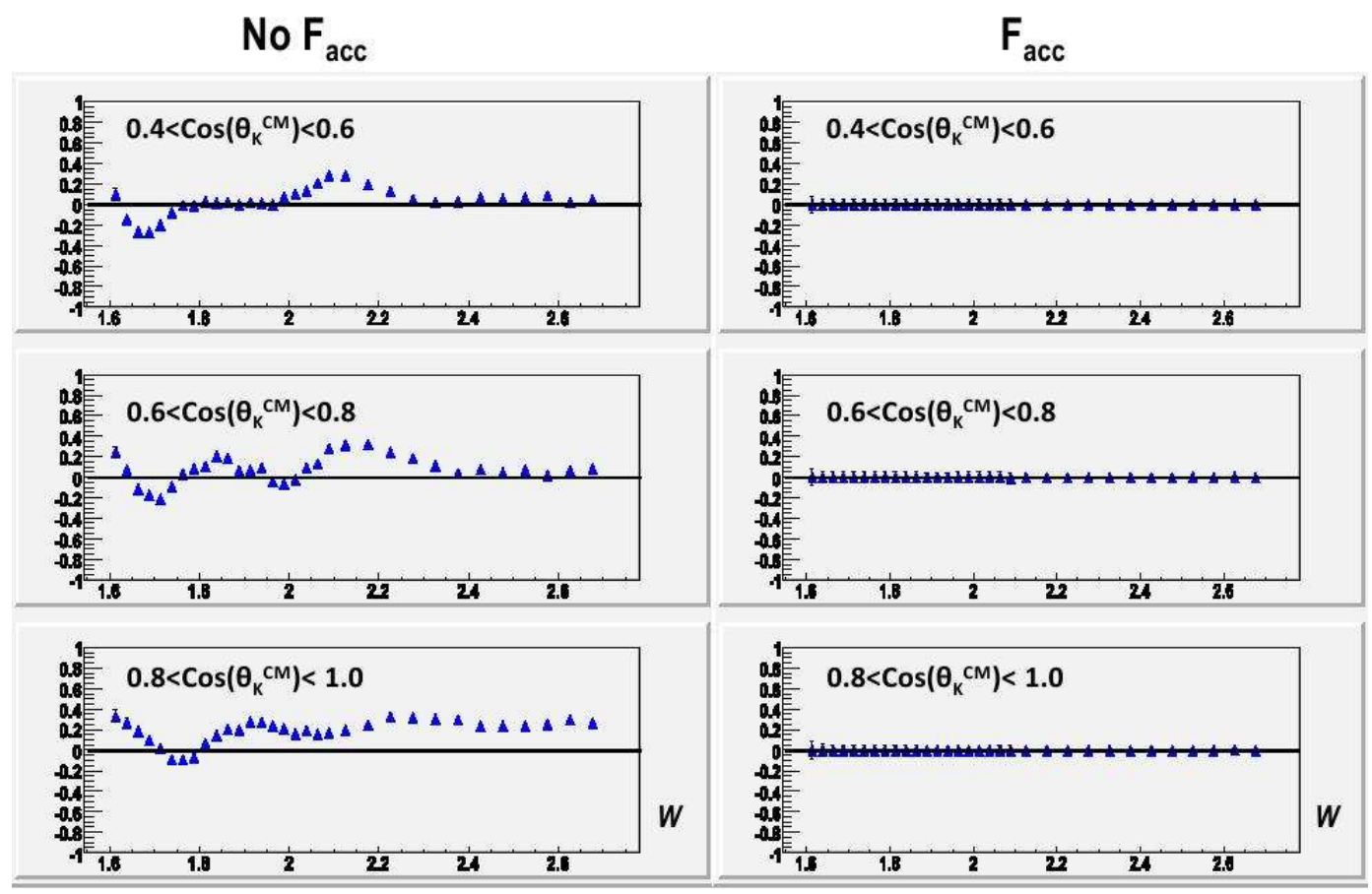

Figure 4.12: $P_{L}$ extracted from the MC dataset as a cross check of the procedure. The left column shows the results without acceptance corrections. Non-zero polarization in these plots is the result of the acceptance effects. The right column shows the results after applying the acceptance corrections. As expected, the polarization is back to zero.

code used for the data analysis. Since the GSIM assumes perfect detector systems for CLAS, some of the corrections applied in the data analysis, the momentum corrections in particular, are omitted when analyzing the simulated data.

The acceptance factors in this analysis are defined as the ratio of the reconstructed events to the generated events in the same kinematic bin. Two acceptance factors $f_{ \pm}$ are defined in each kinematic bin corresponding to forward and backward $p$ angular distributions according to:

$$
f_{ \pm}=\frac{N_{\text {Detected }}^{ \pm}}{N_{\text {Thrown }}^{ \pm}} .
$$

The numerator $N_{\text {Detected }}^{ \pm}$is the sum of the $\Lambda$ missing mass histogram entries within the appropriate range for reconstructed events. The generated or thrown events in each kinematic bin are calculated by filling a counter histogram using the $K^{+}$and $\Lambda$ PDG particle identification codes. Events are generated allowing uniform $\Lambda$ decay, in which 
case the number of the thrown events are treated to be equal both for forward and backward $p$ angular bins. In other words, $\Lambda$ 's are produced with zero polarization in the MC dataset. The denominator $N_{\text {Thrown }}^{ \pm}$in Eq. 4.11 is calculated using one half of all thrown events. The statistical uncertainties on acceptance the factors are given by:

$$
d f_{ \pm}=\sqrt{\frac{f_{ \pm}\left(1-f_{ \pm}\right)}{N_{\text {thrown }}^{ \pm}}} .
$$

Some cross checks are performed to make sure that the acceptance factors are fully understood. Fig. 4.12 shows the $W$ dependence of the $P_{L}$ component of the induced polarization extracted from the $\mathrm{MC}$ data for three $\cos \theta_{K}^{C M}$ bins before and after applying the acceptance corrections. This figure shows that the non-zero polarization along the longitudinal axis is a result of detector acceptance effects. Applying the acceptance correction brings the polarization back to zero, as expected. The $W$ dependence of the acceptance factors are plotted in Fig. 4.13 for the most forward kaon angular bin. As can be seen from the plot, the normal component of the polarization has nearly identical acceptances for both forward and backward going protons, while the other two components have large differences in the forward and backward acceptances and are therefore more sensitive to acceptance effects. This statement is true for all $\cos \theta_{K}^{C M}$ angles. The acceptance corrected yields for the forward and backward directions are given by:

$$
n_{ \pm}=N_{ \pm} / f_{ \pm}
$$

Here, the $N_{ \pm}$are the background subtracted, uncorrected yields, obtained by fitting as described in Section 4.4 and the $f_{ \pm}$are the acceptance correction factors from Eq. 4.11. By propagating the uncertainties $d N_{ \pm}$(Eq. 4.10) and $d f_{ \pm}$(Eq. 4.12), the uncertainties on the corrected yields $n_{ \pm}$are calculated according to:

$$
d n_{ \pm}=n_{ \pm} \sqrt{\left(\frac{\delta N_{ \pm}}{N_{ \pm}}\right)^{2}+\left(\frac{\delta f_{ \pm}}{f_{ \pm}}\right)^{2}} .
$$



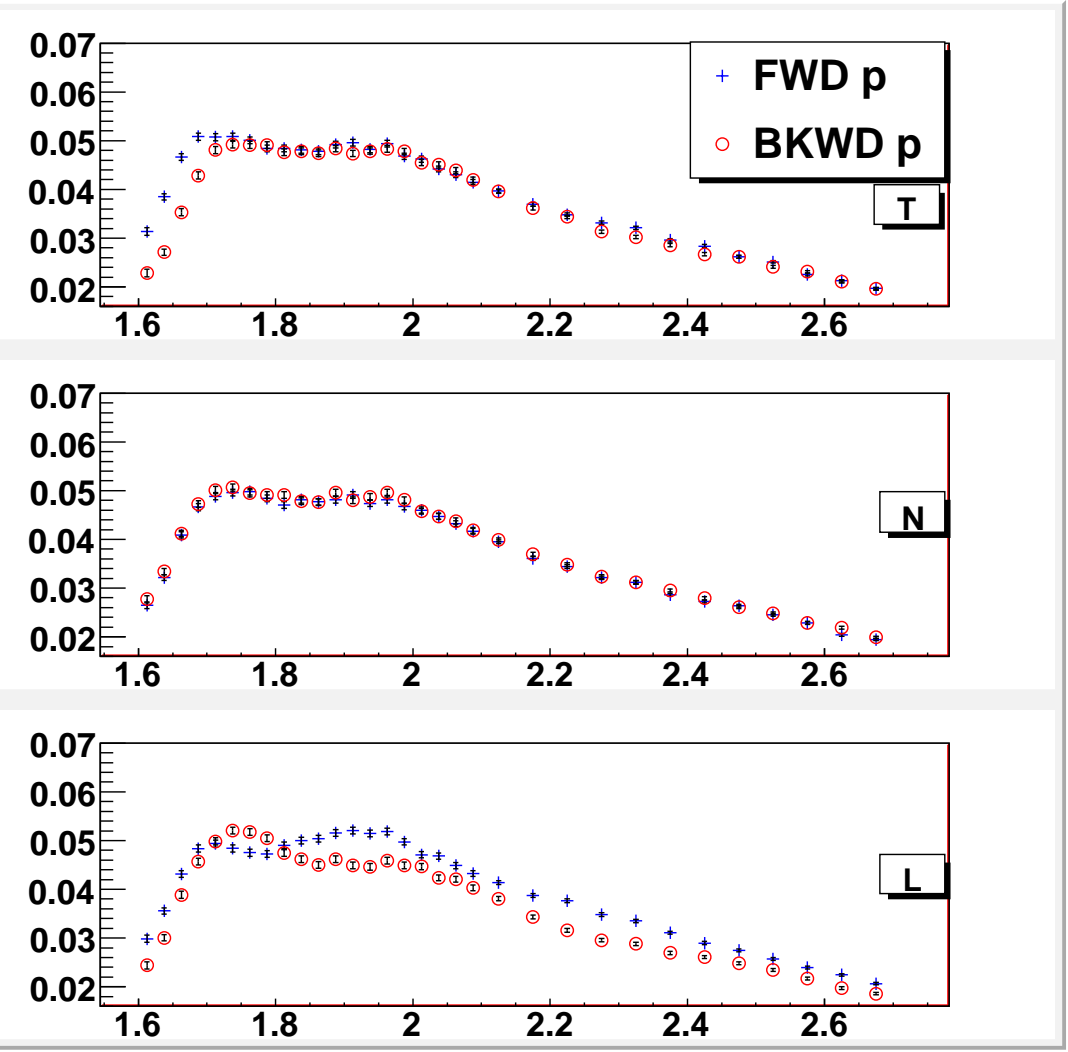

Figure 4.13: Dependence of acceptance factors on $W$ for forward and backward going protons at $0.8<\cos \theta_{K}^{C M}<1$ with respect to the $\mathrm{T}, \mathrm{N}$, and $\mathrm{L}$ axes. The acceptance factors for forward and backward going protons for the $\mathrm{N}$ component have practically identical dependence on $W$, while for the $\mathrm{T}$ and L components they strongly differ.

\subsection{Recoil Polarization Calculation}

After all final state particles of interest are identified, all kinematic quantities are determined in the lab frame. However, the proton angular distributions must be calculated in the $\Lambda$ rest frame. A set of Lorentz transformations are applied to find the energies and momenta of all final state particles in the center of momentum frame $(\mathrm{CM})$ of the virtual photon and the target proton. Theses quantities are then fed to a routine, which performs the transformations to the $\Lambda$ rest frame and finds the proton angular distributions in that frame relative to the different spin quantization axes ( $T, N, L)$. The background subtracted yields, obtained by fitting the $\Lambda$ missing mass histograms for forward and backward $p$ angles (Section 4.4) are then corrected for the acceptance. The 
induced polarization of the recoil $\Lambda$ is extracted via the forward-backward asymmetry from the $p$ angular distributions with respect to $\cos \theta_{p}^{R F}=0$ as described in Section 1.4.

\section{Forward-Backward Asymmetry}

The induced polarization in this case is given by:

$$
P=\frac{2}{\alpha} A=\frac{2}{\alpha}\left(\frac{n_{+}-n_{-}}{n_{+}+n_{-}}\right)
$$

where $A=\frac{n_{+}-n_{-}}{n_{+}+n_{-}}$is forward-backward asymmetry, $n_{ \pm}$are the acceptance-corrected yields in the forward and backward directions, respectively, given by Eq. 4.13. In this equation $\alpha=0.642 \pm 0.013$ is the $\Lambda$ decay asymmetry parameter and is taken from the PDG [7]. The uncertainty in $\alpha$ is treated as systematic uncertainty and is included in the total systematic uncertainty calculations as summarized in Table 5.1. A careful propagation of errors is necessary in order to calculate the error bars on the polarization results. First, the uncertainties on the forward-backward asymmetry $A$ are determined by:

$$
d A^{2}=\left(\frac{\delta A}{\delta n_{+}} d n_{+}\right)^{2}+\left(\frac{\delta A}{\delta n_{-}} d n_{-}\right)^{2} .
$$

The partial derivatives are calculated:

$$
\begin{aligned}
\frac{\delta A}{\delta n_{+}} & =\frac{2 n_{-}}{\left(n_{+}+n_{-}\right)^{2}}, \\
\frac{\delta A}{\delta n_{-}} & =\frac{-2 n_{+}}{\left(n_{+}+n_{-}\right)^{2}} .
\end{aligned}
$$

Substituting these partial derivatives into Eq. 4.16 will give:

$$
d A^{2}=\frac{4\left(n_{-} d n_{+}\right)^{2}}{\left(n_{+}+n_{-}\right)^{4}}+\frac{4\left(n_{+} d n_{-}\right)^{2}}{\left(n_{+}+n_{-}\right)^{4}} .
$$


The respective statistical uncertainty in the polarization is given by:

$$
d P=\frac{2 d A}{\alpha}=\frac{4}{\alpha\left(n_{+}+n_{-}\right)^{2}} \sqrt{\left(n_{-} d n_{+}\right)^{2}+\left(n_{+} d n_{-}\right)^{2}}
$$

where $d n_{ \pm}$is determined according to Eq. 4.14

\subsection{Radiative Corrections}

In the electroproduction experiments, in general, it is necessary to take into account so called radiative effects. While moving in the electromagnetic field of the nucleus, the electron can emit or absorb a virtual photon before or after interacting with the target proton or produce electron-positron pairs. Speaking in the language of Feynman diagrams, the radiative corrections correspond to loop or higher order diagrams. These corrections are calculated via perturbation theory in QED, where the expansion parameter is proportional to $\alpha^{2}$ ( $\alpha=\frac{1}{137}$ is the fine structure constant), so that each next higher order contribution is roughly $10^{-4}$ times less than the previous one.

The result of photon emission by electrons, before or after interacting with the target proton, shows up as a radiative tail in the higher mass range of the $\Lambda$ missing mass histograms. The radiative tails of the $\Lambda$ and $\Sigma^{0}$ peaks were not well constrained in the fits because of the low statistics, especially in the high $W$ bins. A tight hyperon missing-mass cut is applied while extracting the uncorrected yields in order to exclude the radiative tails. As part of the systematic uncertainty analysis, the missing mass cut was removed, allowing the tails to be included in the yields. The study showed (Section 5.1) that excluding the tail events does not introduce any major systematic uncertainty $(<0.026)$. Actually, the radiative effects effectively cancel out in the asymmetry approach, used for polarization calculations described in Section 4.6. Based on these facts the radiative corrections were omitted in this analysis. 


\begin{tabular}{|r|r|r|r|}
\hline Variable & Range & \# of bins & Bin Width \\
\hline \hline $\cos \theta_{K}^{C M}$ & $(-1.0,0.0)$ & 1 & 1. \\
& $(0.0,0.4)$ & 1 & 0.4 \\
& $(0.4,0.8)$ & 1 & 0.4 \\
& $(0.8,1.0)$ & 1 & 0.2 \\
\hline$W$ & $1.6-2.4 \mathrm{GeV}$ & 4 & $200 \mathrm{MeV}^{2}$ \\
\hline$Q^{2}$ & $0.8-3.2 \mathrm{GeV}^{2}$ & 4 & $0.6 \mathrm{GeV}^{2}$ \\
\hline
\end{tabular}

Table 4.8: Binning for the $Q^{2}$ dependence studies.

\section{8 $\quad Q^{2}$ Dependence Study}

Initially, a $Q^{2}$ study was performed for data integrated over all $\cos \theta_{K}^{C M}$ and $\phi_{K}$ angles. When integrated over $\cos \theta_{K}^{C M}$, the results are dominated by forward angle ( $t$-channel) data due to the strong forward peaking of the data. Results showed no $Q^{2}$ dependence. Since the resonance contributions to polarization observables are expected to be visible at medium $\cos \theta_{K}^{C M}$ angles ( $s$-channel process), the $Q^{2}$ dependent analysis was repeated in the last stage for several $\cos \theta_{K}^{C M}$ bins. The binning is shown in Table 4.8.

The polarization vs. $Q^{2}$ plots are shown in Fig. 4.14. The results again show no $Q^{2}$ dependence over the entire range covered by the E1F dataset. This fact allows one to integrate data over this variable for the final analysis without losing any valuable information. Although the data are integrated over $Q^{2}$, electroproduction still provides valuable information such as access to the interference response functions, which is not possible via photoproduction. 
$-1.0<\cos \left(\theta_{k}{ }^{C M}\right)<0$.
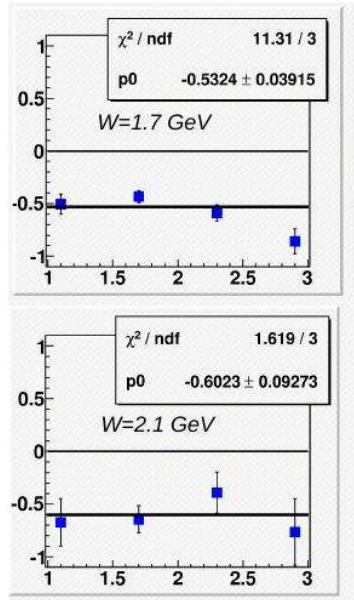

$0.4<\cos \left(\theta_{\mathrm{K}}{ }^{\mathrm{CM}}\right)<0.8$
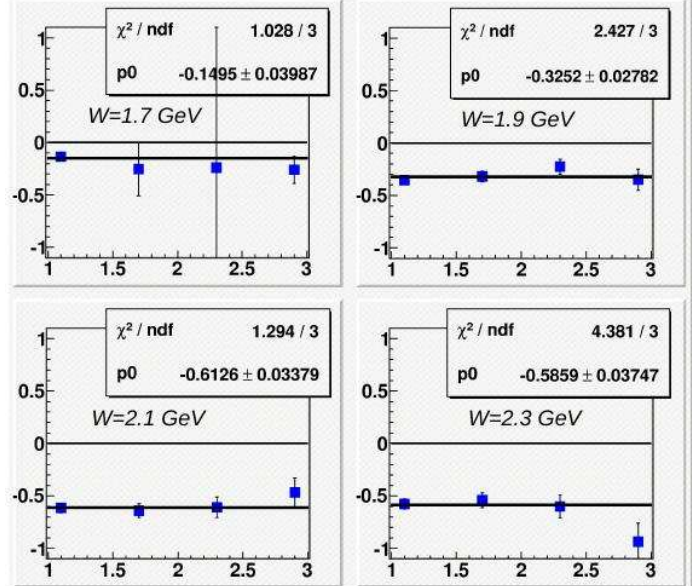

$0 .<\cos \left(\theta_{K}{ }^{C M}\right)<0.4$
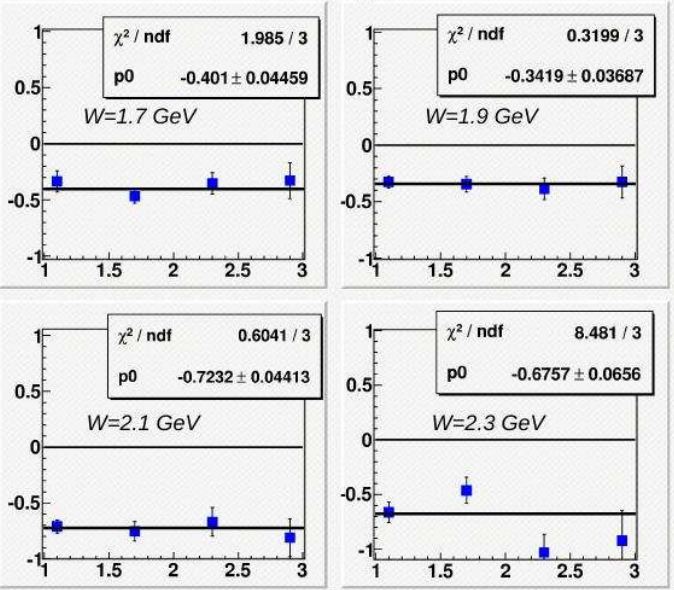

$0.8<\cos \left(\theta_{\mathrm{K}}{ }^{\mathrm{CM}}\right)<1.0$

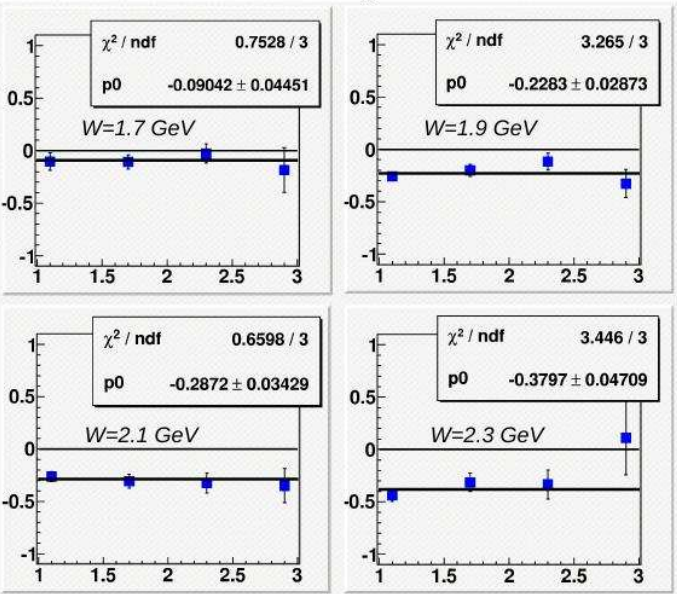

Figure 4.14: $P_{N}$ vs. $Q^{2}$ for different $\cos \theta_{K}^{C M}$ and $W$ bins. The results show no significant dependence on $Q^{2}$ within our statistical uncertainties. 


\section{CHAPTER 5}

\section{Systematic Uncertainties}

There are several sources of systematic uncertainties that have to be taken into account in this analysis. These sources are categorized as:

1. Polarization Extraction

2. Acceptance

3. Background Subtraction

The systematic uncertainties are assigned for each source, comparing polarization results in each $\cos \theta_{K}^{C M}$ kinematic bin with nominal and alternative cuts. The systematic uncertainties are estimated as the uncertainty-weighted average polarization defined by:

$$
\langle P\rangle=\sqrt{\frac{\sum_{i=1}^{n} \frac{\left[P_{i}^{\text {nom }}-P_{i}^{\text {alt }}\right]^{2}}{\left(\delta P_{i}^{\text {nom }}\right)^{2}}}{\sum_{i=1}^{n} \frac{1}{\left(\delta P_{i}^{\text {nom }}\right)^{2}}}} .
$$

Here the summation goes over all $W$ points for each $\cos \theta_{K}^{C M}$ bin. The RMS width of the uncertainty-weighted polarization differences are also calculated as a cross check of the procedure. The systematic uncertainties from all sources is summarized in Table 5.1. The total systematic uncertainty in each $\cos \theta_{K}^{C M}$ kinematic bin is obtained by summing the uncertainties from all sources in quadratures since the sources are assumed to be uncorrelated.

\subsection{Acceptance Corrections}

The uncorrected yields are integrated within a specific $\Lambda$ missing mass window. The nominal missing mass integration range is from 1.05 to $1.15 \mathrm{GeV}$. Systematic uncer- 


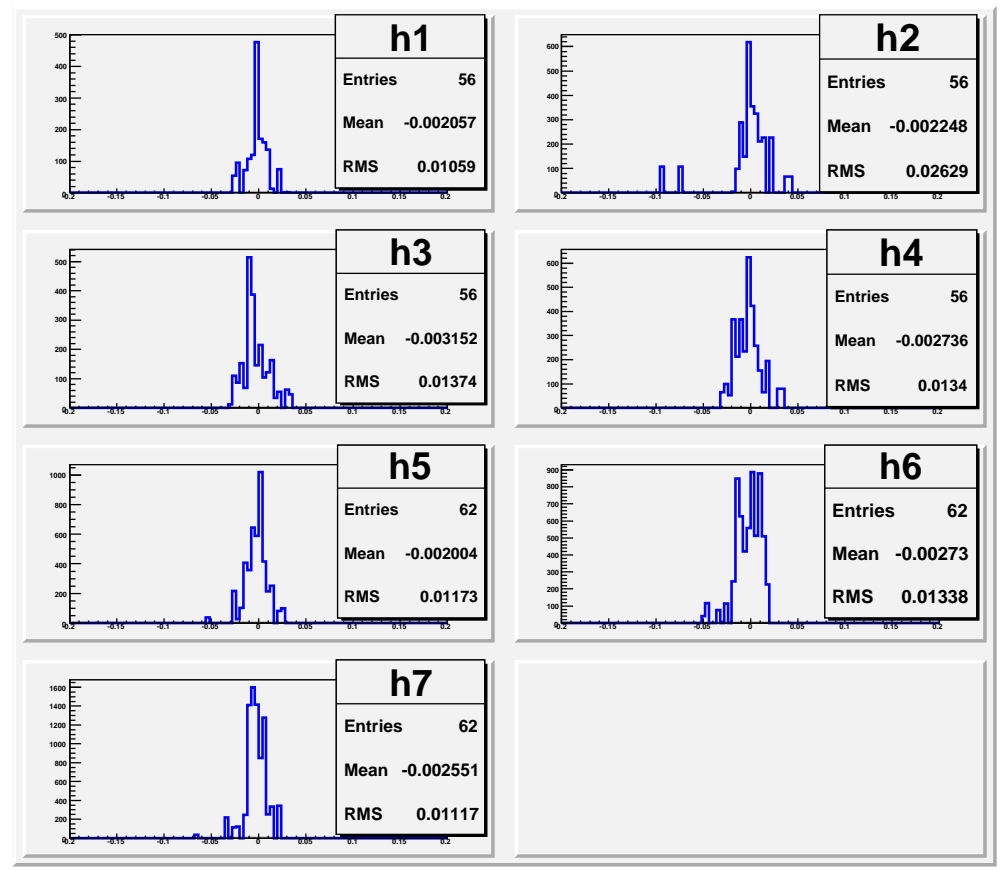

Figure 5.1: Uncertainty-weighted histograms for the polarization differences with the nominal and alternative cuts for each $\cos \theta_{K}^{C M}$ bin. These histograms combine the two cases of alternative missing mass integration ranges. The RMS values of these histograms are the assigned systematic uncertainty for each $\cos \theta_{K}^{C M}$ bin.

tainties from this source are studied for two alternative ranges: 1) from 1.025 to 1.17 $\mathrm{GeV}$, and 2) from 0.9 to $1.4 \mathrm{GeV}$ which is the total range used for the fits. Strictly speaking, extending the integration range does not affect the experimental yields, since the amplitudes of the $\Lambda$ fit functions do not change once the fitting procedure is complete, but the acceptance factors do. Extending the range of integration affects the polarization results, because this integration must also be taken into account in the acceptance factor calculations, when integrating the MC $\Lambda$ templates. Correcting the yields for the acceptance gives rise to small systematic uncertainties. The RMS width of the uncertaintyweighted polarization differences, with the nominal and alternative cuts in place, is read directly from the histograms (Fig. 5.1) as the measure of the systematic uncertainties from this source. Histograms combine both alternative cases. The estimated absolute uncertainties from this source range from 0.011 to 0.026 . 


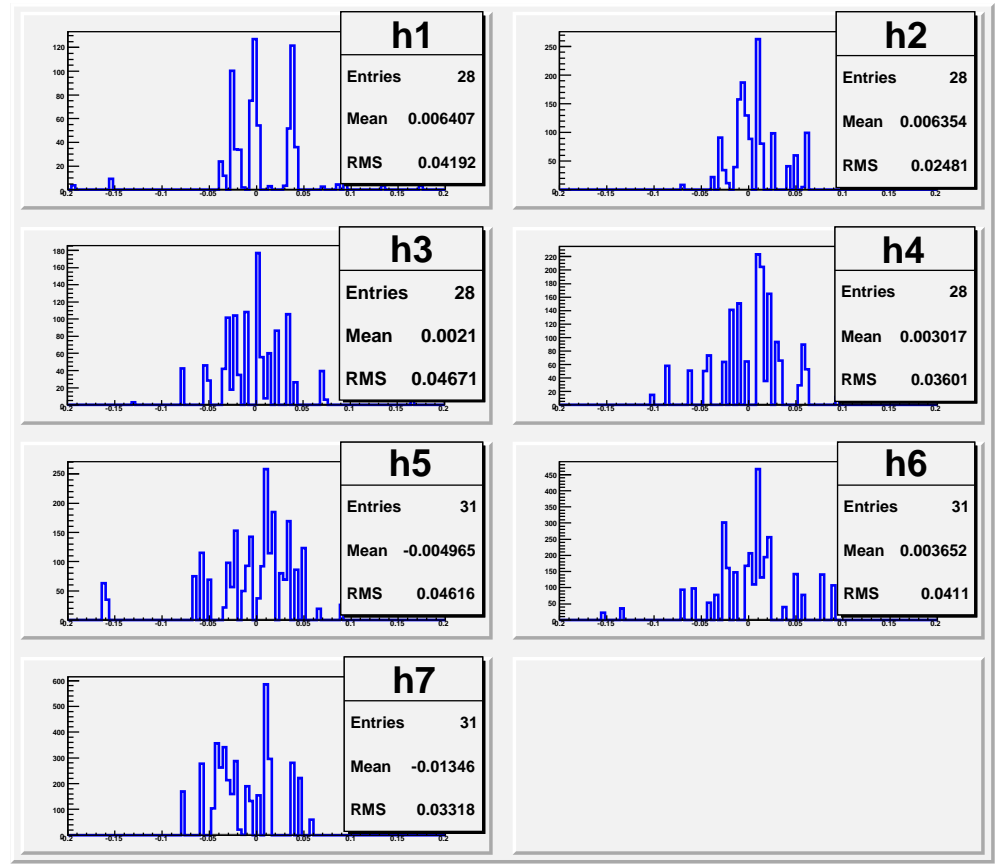

Figure 5.2: Uncertainty-weighted histograms for the polarization differences with the nominal and alternative pion missing mass squared cuts. The systematic uncertainties are the RMS values of these histograms, assigned for each $\cos \theta_{K}^{C M}$ bin.

\subsection{Pion Missing Mass Cut}

The applied nominal pion missing mass squared cut is from -0.02 to $0.07 \mathrm{GeV}^{2}$. The alternative cut is extended from -0.03 to $0.1 \mathrm{GeV}^{2}$. This lets in some additional background events, potentially changing the background templates. Fig. 5.2 shows the uncertainty-weighted histograms for the polarization differences with the applied nominal and alternative cuts. The estimated absolute uncertainties from this source range from 0.025 to 0.047 .

\subsection{Geometrical Fiducial Cuts}

The nominal geometrical fiducial cuts applied for this analysis are the loose cuts for both $e^{-} \mathrm{s}$ and hadrons. As an alternative, to study the systematic effects, tighter fiducial cuts were used. The parameters are summarized in Tables 3.1 and 3.2. The applied 

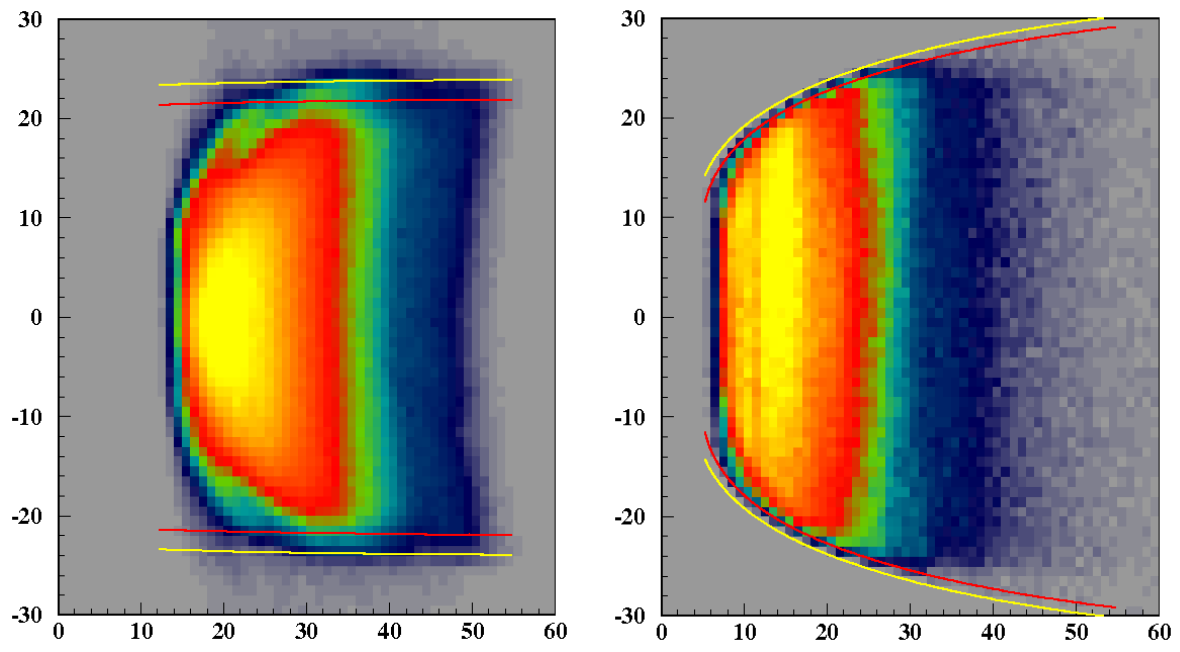

Figure 5.3: $\phi$ vs. $\theta$ distributions for electrons (left) and kaons (right). The yellow and red lines show the limits of the loose and medium fiducial cuts, respectively.

cuts are shown in Fig. 5.3 both for electrons (left) and kaons (right). The yellow and red lines show the limits of the loose and medium fiducial cuts, respectively. Since the fiducial cuts as defined in Sections 3.1 and 3.2 depend on momentum, $p_{e}=1.1 \mathrm{GeV}$ and $p_{K}=1.1 \mathrm{GeV}$ are selected to display the cuts. The uncertainty-weighted histograms for the polarization differences with the nominal and alternative cuts are illustrated in Fig. 5.4. The estimated absolute uncertainties from this source range from 0.040 to 0.080 .

\subsection{Proton Acceptance Corrections with Polarized MC}

The $e^{-}$and $K^{+}$acceptance effects are effectively canceling out in the forward-backward asymmetry calculations used for the polarization extraction, but the $p$ acceptance does not and must be carefully studied.

This section describes the systematic impact of the proton acceptance effects on our results. The Monte Carlo dataset used for the acceptance corrections was generated with the assumption that the $\Lambda$ decays uniformly into $p \pi^{-}$. Here we study the acceptance effects with different cases of polarizations in the MC sample. Instead of generating 


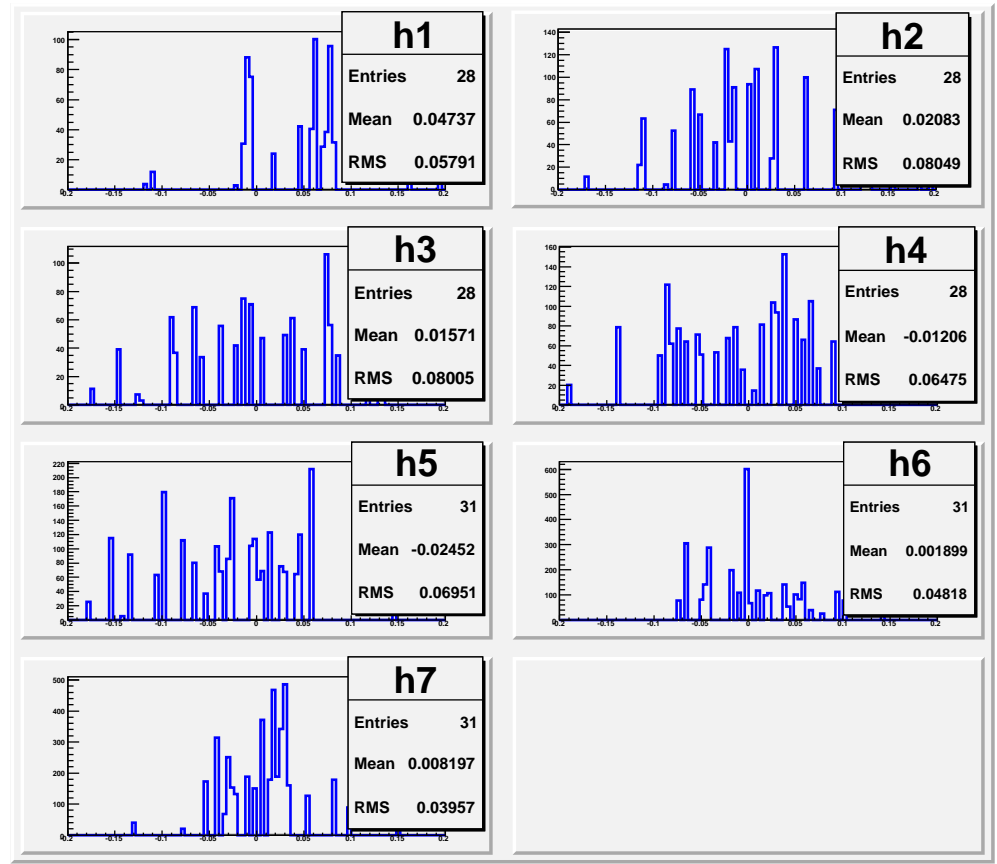

Figure 5.4: Uncertainty-weighted histograms for the polarization differences with the nominal (loose) and alternative (tighter) fiducial cuts. The RMS values of these histograms again are the systematic uncertainties for a given $\cos \theta_{K}^{C M}$ bin.

new MC datasets, the decay proton flat angular distributions are scaled by a factor $f$ :

$$
f=1+\alpha P \cos \theta_{p}^{R F}
$$

The systematic uncertainties associated with this source are studied for three different cases of polarizations: $P=-0.55 ; P=+0.55 ; P=P_{\text {nominal }}$, where $P_{\text {nominal }}$ is our final measured polarizations. Several cross checks were performed in order to make sure that the acceptance factors are fully understood. The angular distributions and polarizations are extracted from the MC dataset both for the generated and reconstructed protons after scaling. Fig. 5.5 illustrates the $p$ angular distributions. The polarization values shown in these plots are calculated from the slope of the linear fits. The $W$ dependence of the polarization, extracted from the MC dataset after scaling $(P=-0.55$ case), is shown in Fig. 5.6. The top plot demonstrates the polarization results extracted directly from the generated $p$ information. The plot in the middle shows the polariza- 

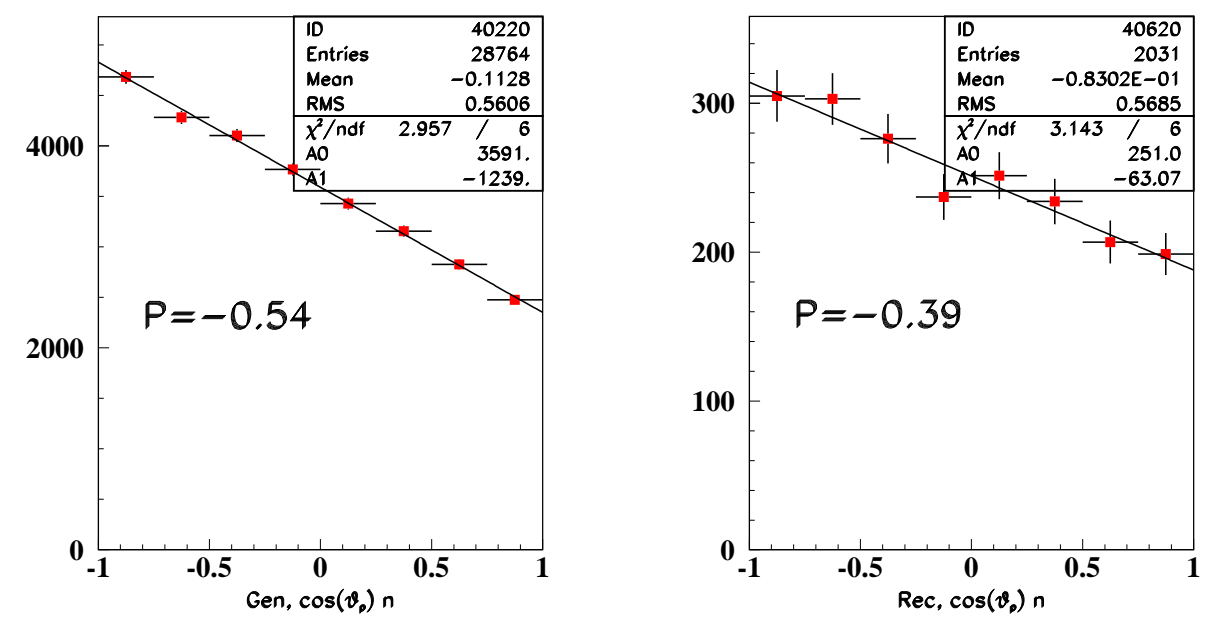

Figure 5.5: Decay proton angular distributions extracted from the MC dataset after scaling by the factor $f$ given by Eq. 5.2 for the case of $P=$ -0.55 . The left plot corresponds to the generated and the right plot to the reconstructed protons. The polarization values shown on the plots are calculated from the slope of the linear fit.

tions extracted from the reconstructed $p$ information without any acceptance corrections. Finally, the results in the bottom plot are the acceptance corrected polarizations. As one can see from these plots, applying the acceptance corrections reproduces the polarization values $P=-0.55$ within statistical uncertainties, by which the events were scaled. Similar cross-checks were done also for the other two cases.

The final systematic uncertainty from this source again is the RMS of the uncertaintyweighted polarization differences between the nominal results and the polarizations calculated with the three different sets of acceptance factors, extracted from the MC dataset after scaling by the factor $f$ given by Eq. 5.2. The histograms in Fig. 5.7 combine all three cases of the polarization values listed above that were used for scaling. The estimated absolute uncertainties from this source range from 0.064 to 0.093 . 


$$
\begin{gathered}
\text { Weighted histograms: } 1+\alpha \mathrm{P} \cos \left(\theta_{p}^{R F}\right)=1-0.3531 \cos \left(\theta_{p}^{R F}\right) \\
\alpha=0.642, P=-0.55
\end{gathered}
$$

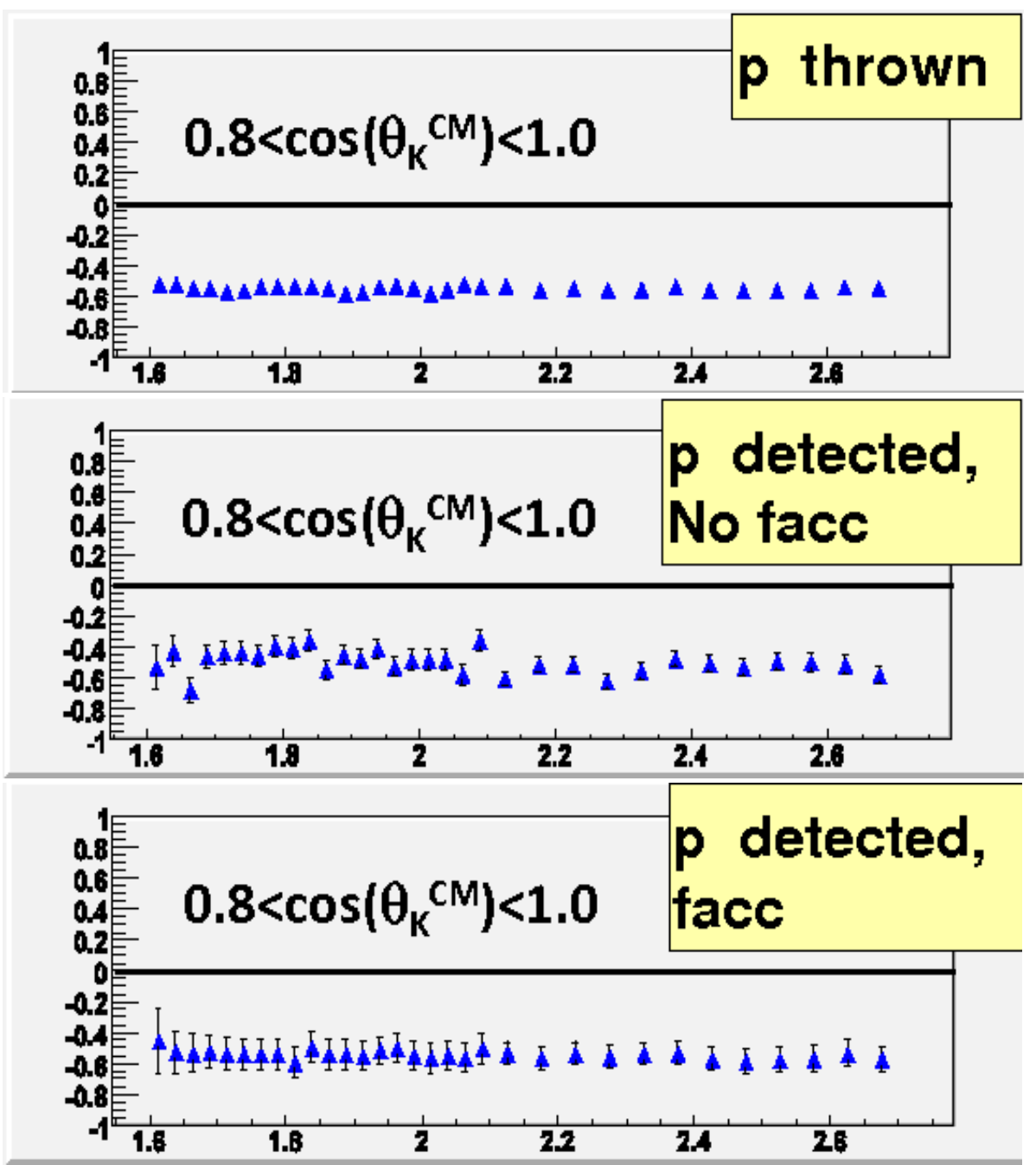

Figure 5.6: Polarization versus $W$ extracted from the $\mathrm{MC}$ dataset after scaling $(P=-0.55$ case $)$ as a cross-check of the procedure. The top plot results are obtained from the generated proton information. The middle plot polarizations are for reconstructed protons but with no acceptance corrections applied. Finally, the polarizations in the bottom plot are for the reconstructed protons after applying the acceptance corrections. The $P=-0.55$ value is reproduced within statistical uncertainties. 


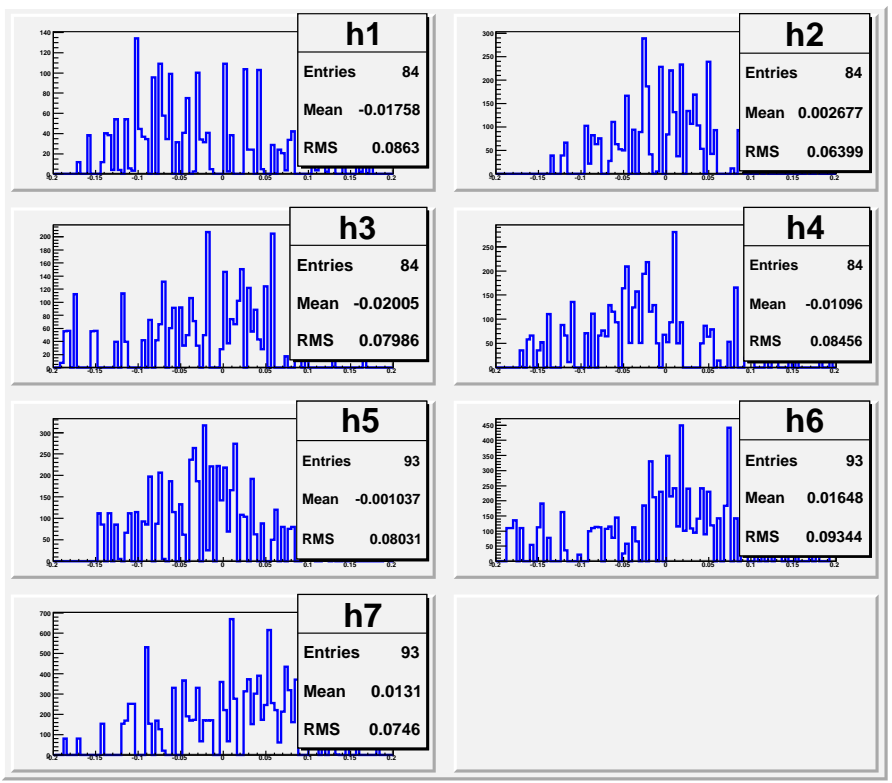

Figure 5.7: Uncertainty-weighted histograms for the polarization differences between the nominal results and the polarizations calculated with the three different sets of acceptance factors extracted from the MC dataset after scaling by the factor $f$ given by Eq. 5.2 for all three cases of the polarization values. The assigned systematic uncertainties for each $\cos \theta_{K}^{C M}$ bin are the RMS values taken directly from these histograms.

\section{$5.5 \quad t$-Slope}

The MC dataset used for the acceptance corrections was generated with a $t$-slope $=0.3$ $\mathrm{GeV}^{-2}$. Two small MC datasets, with $\sim 10 \mathrm{M}$ events each, were generated with $t$-slopes $=0.1$ and $1.0 \mathrm{GeV}^{-2}$ as part of the systematic uncertainty analysis. Increasing the $t$ slope in the generator forces more kaons to go in the forward direction, thus sending protons into different parts of the detector. These two sets are then used for acceptance corrections. The histogram of uncertainty-weighted polarization differences is shown in Fig. 5.8 combining both cases. The estimated absolute uncertainties from this source range from 0.063 to 0.082 . Since the $t$-slope studies also reflect the proton acceptance corrections, no separate $t$-slope systematic uncertainties are included in the final systematic uncertainty calculations. 


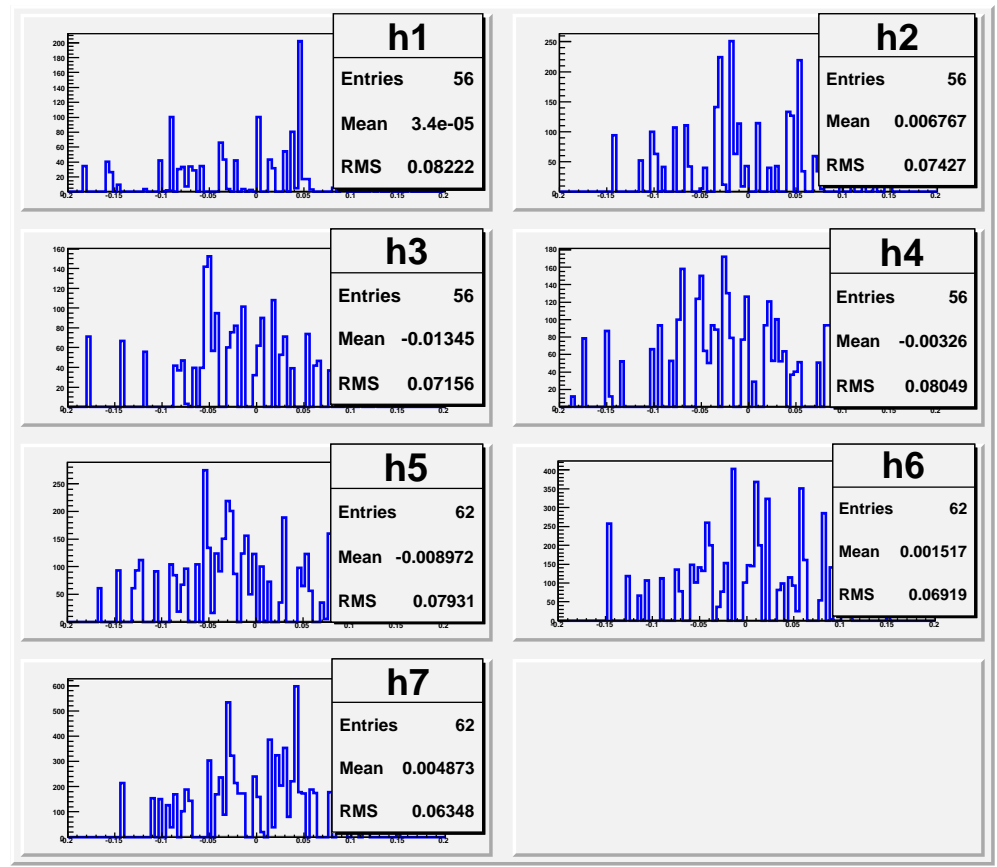

Figure 5.8: Uncertainty-weighted histograms for the polarization differences combining the different cases of $t$-slope values. The RMS values are the assigned systematic uncertainties.

\subsection{Fit Method}

The fits in the background subtraction procedure were performed by two methods. The first method was the PAW fitting routine described in Section 4.4. The second method is a stand alone FORTRAN code, which performs the combined fits of the missing mass histograms for all three polarization components for both forward and backward moving protons, using the same functional form for the $\Lambda, \Sigma^{0}$, and background templates as the first method. To estimate the uncertainties associated with the fitting procedure, in this section the results with eight parameter fits by both fitting methods are compared. This means that the $\Lambda$ Lorentzian parameters are allowed to vary in addition to the absolute amplitudes. Fig. 5.9 shows the histogram of uncertainty-weighted polarization differences. The assigned systematic uncertainty is the RMS of the histogram. The estimated absolute uncertainties from this source range from 0.030 to 0.052 . 


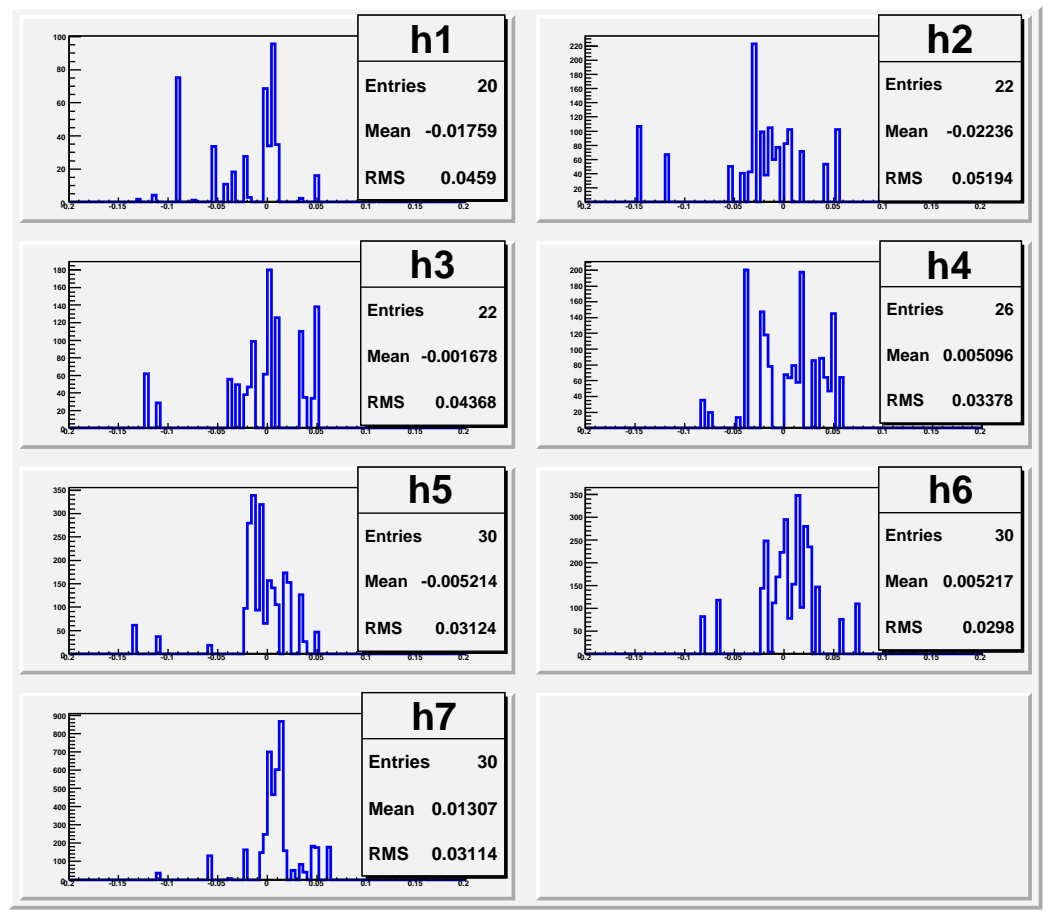

Figure 5.9: Uncertainty-weighted histograms for the polarization differences extracted by the two fit methods (Section 5.6). The RMS values are the assigned systematic uncertainties.

\subsection{Fixed vs. Float Fit Parameters}

This section presents the systematic effects on the polarization results for the two different assumptions of the fit parameters. The nominal results are obtained with a 6parameter fit, where all shape parameters are fixed or parametrized as a function of $W$, while the amplitudes are allowed to vary freely. As an alternative fit, the $\Lambda$ Lorentzian parameters are allowed to the float in addition to amplitudes. Fig. 5.10 shows the histogram of the uncertainty-weighted polarization differences for these two cases. The estimated absolute uncertainties from this source range from 0.012 to 0.034 .

\subsection{PID Routine}

This section describes the study of systematic uncertainties related to the hadron PID routine. The nominal results are obtained by using the minimum $\Delta t$ method as dis- 


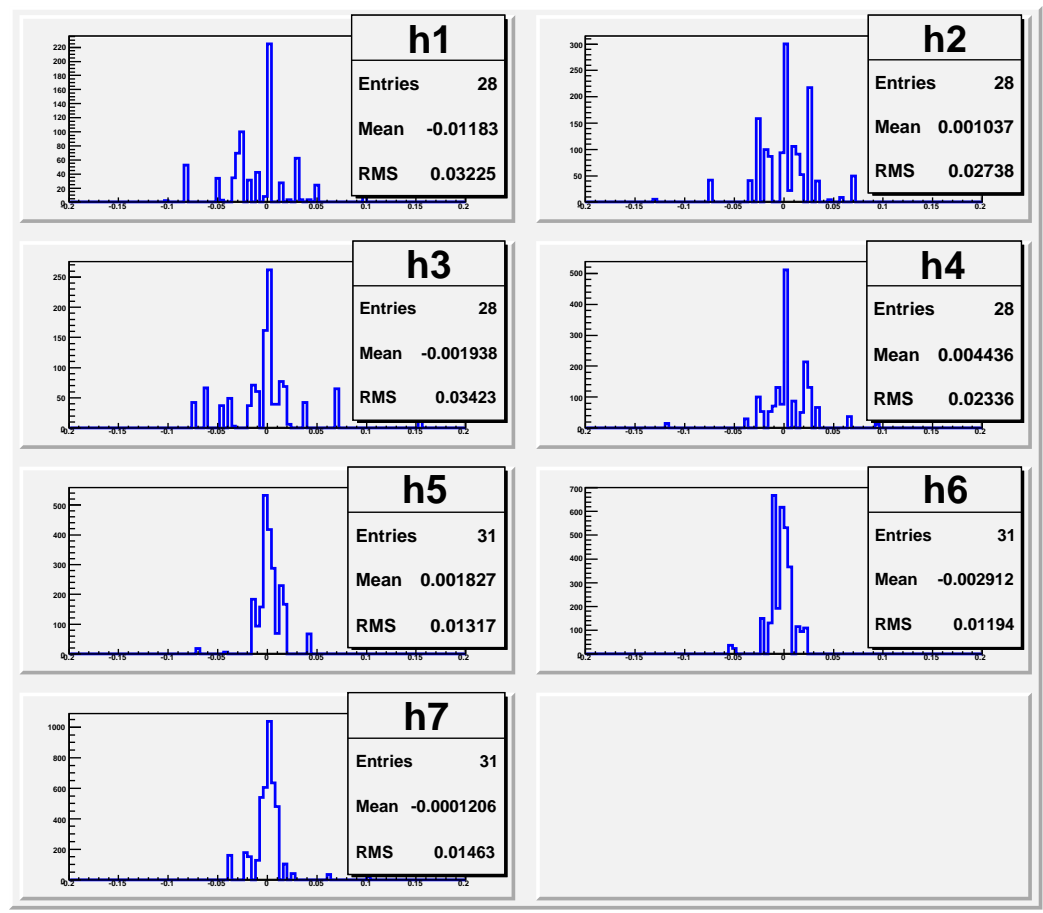

Figure 5.10: Uncertainty-weighted histograms for the polarization differences for the six (all fixed) and eight (floating $\Lambda$ Lorentzian) parameter fits.

cussed in Section 3. The alternative method of hadron identification again uses the timing method, only instead of minimizing $\Delta t$, fixed timing cuts are applied. The results showed that the PID routine is not a source of systematic uncertainties and is therefore not included in the final systematic uncertainty evaluation.

\subsection{Deviations of $P_{L}$ and $P_{T}$ from Zero}

The strongest systematic check of our analysis results, after the $\phi_{K}$ integration and acceptance corrections are applied, are the deviations of $P_{L}$ and $P_{T}$ from zero. These deviations represent the upper limit of the combined systematic uncertainties from all sources. The $W$ dependence of $P_{L}$ and $P_{T}$ components for all kaon angle bins are shown in Figs. 5.11, 5.12. A constant fit to the data gives the average deviations of the polarizations from zero. The results of the constant fits along with their uncertainties are plotted versus $\cos \theta_{K}^{C M}$ in Fig. 5.13 for both $P_{L}$ and $P_{T}$ components. The red box 
in this plot shows the limits corresponding to the minimum total systematic uncertainty from Table 5.1. All deviations within their uncertainties fall within the limits of the calculated total systematic uncertainties.

\subsection{Sector Dependence}

The last cross check is the investigation of sector dependence of the polarization, where the $e^{-}$and $K^{+}$are detected. For this study, the data are sorted with $200 \mathrm{MeV}$ wide $W$ bins at $0.8<\cos \theta_{K}^{C M}<1.0$, where the statistics are the largest. The dependence of $P_{T}$ on $W$ for each $e^{-}$and $K^{+}$sector is shown in Figs. 5.14. Results show no significant sector dependence. No systematic uncertainty is included in the final systematic uncertainty calculations coming from this source. 


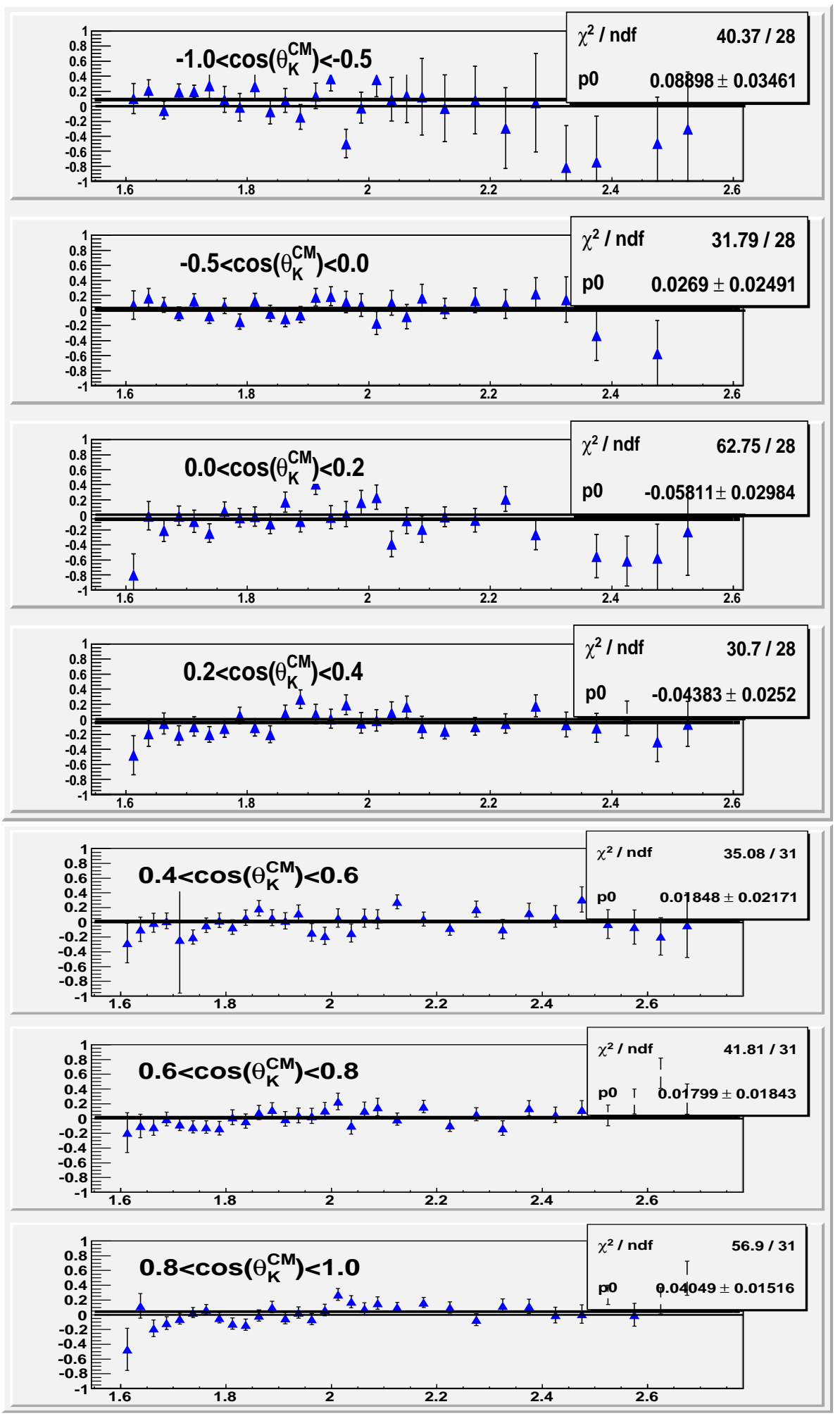

Figure 5.11: $P_{L}$ deviations from zero. The constant fit values on these plots estimate the upper limit on the total systematic uncertainties. 


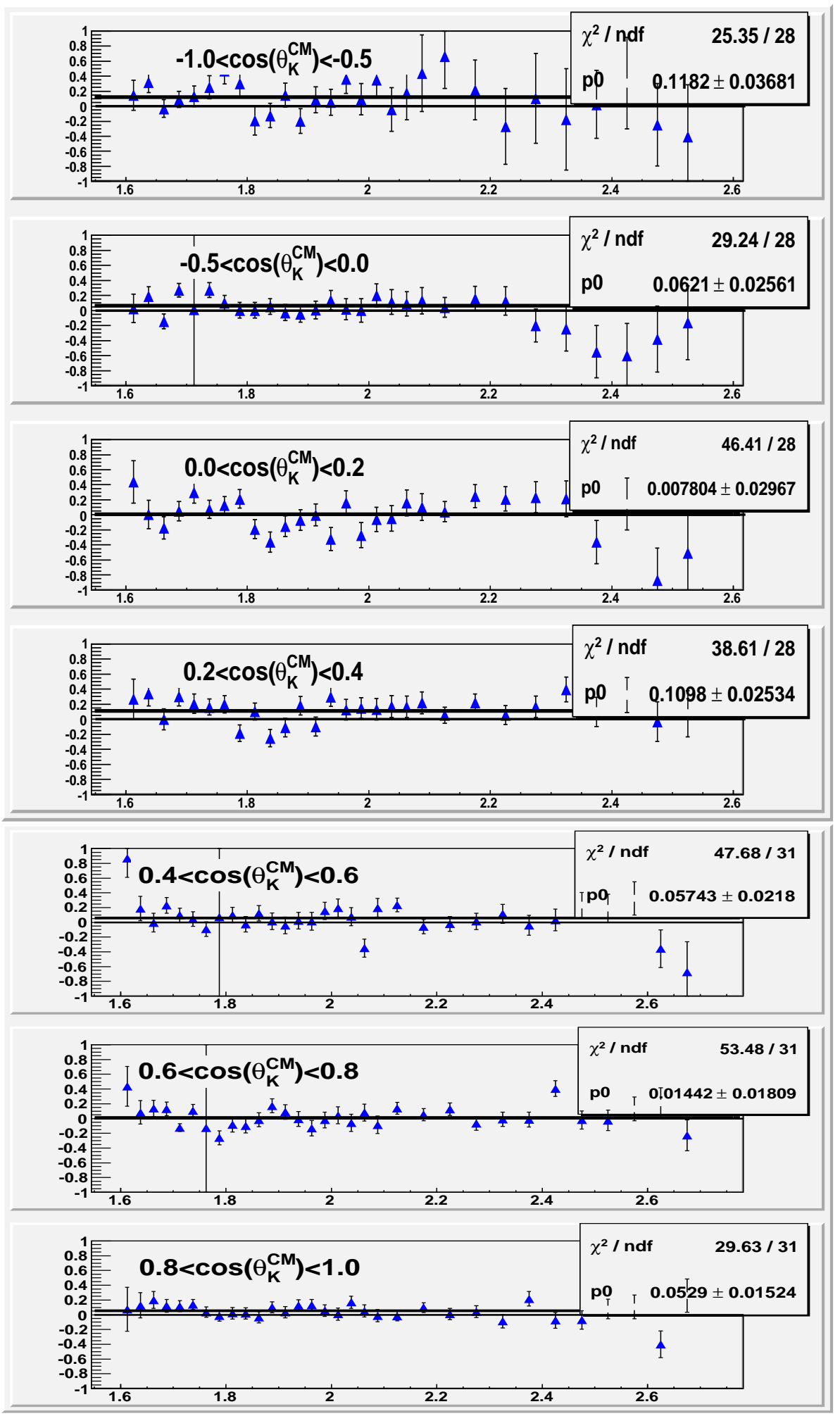

Figure 5.12: $P_{T}$ deviations from zero. The constant fit values on these plots estimate the upper limit on the total systematic uncertainties. 


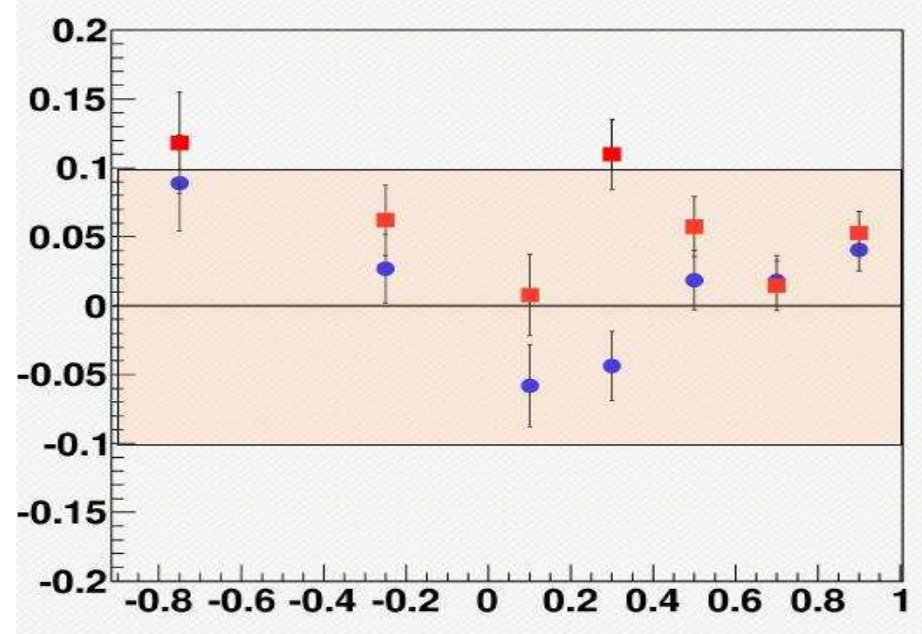

Figure 5.13: Average $P_{L}$ (blue circles) and $P_{T}$ (red squares) deviations from zero vs. $\cos \theta_{K}^{C M}$.
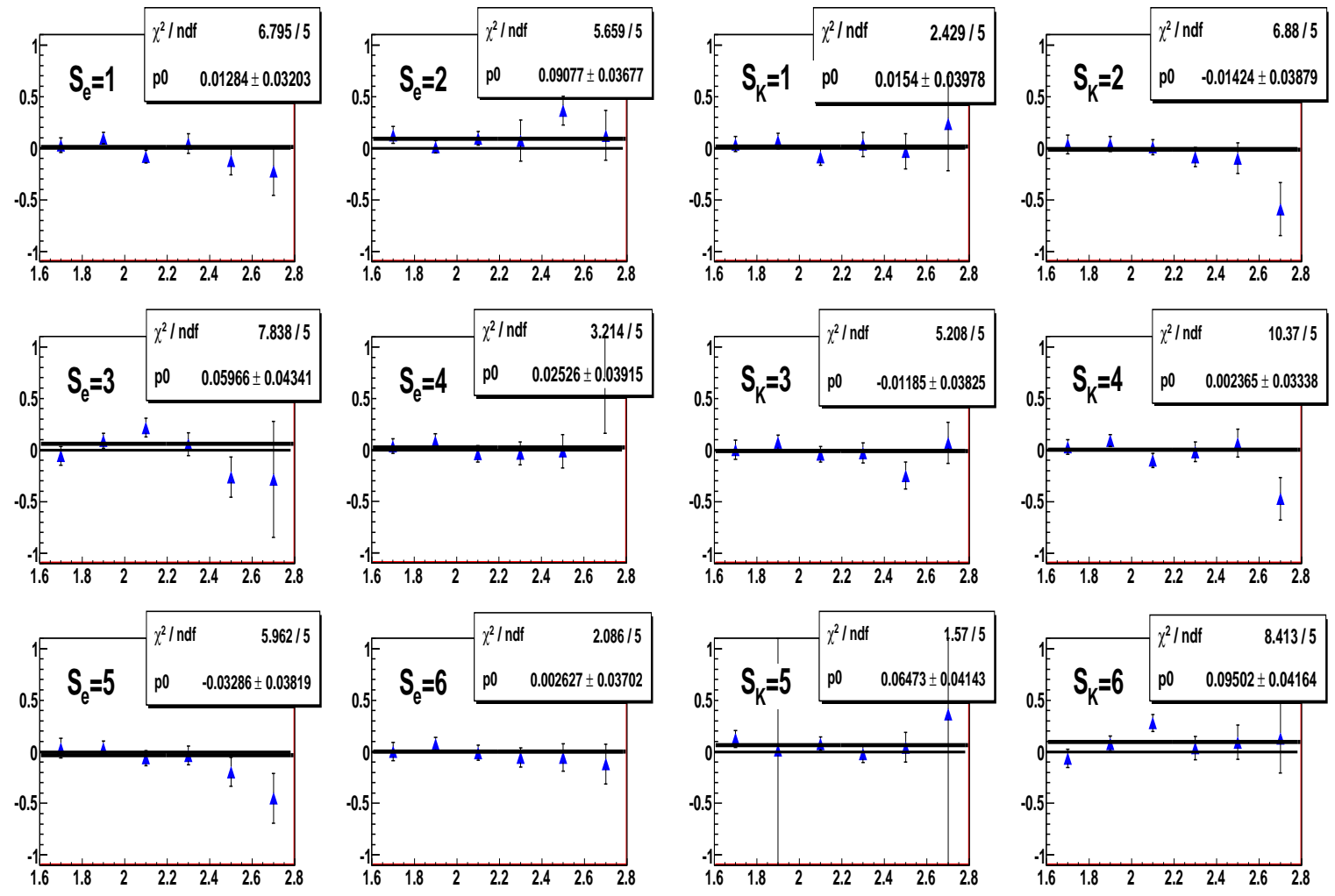

Figure 5.14: $P_{T}$ vs. $W$ at $0.8<\cos \theta_{K}^{C M}<1$ for each $e^{-}$and $K^{+}$sector. The results show no sector dependence. 


\begin{tabular}{|r|r|r|r|r|r|r|r|}
\hline \multicolumn{7}{|c|}{ SYSTEMATICS UNCERTAINTIES } \\
\hline $\cos \theta_{K}^{C M}$ & $(-1.0,-0.5)$ & $(-0.5,0.0)$ & $(0.0,0.2)$ & $(0.2,0.4)$ & $(0.4,0.6)$ & $(0.6,0.8)$ & $(0.8,1.0)$ \\
\hline Acceptance Corrections & 0.011 & 0.026 & 0.014 & 0.013 & 0.012 & 0.013 & 0.011 \\
\hline$M M^{2}\left(e^{\prime} K^{+} p\right)$ & 0.042 & 0.025 & 0.047 & 0.036 & 0.046 & 0.041 & 0.033 \\
\hline $\begin{array}{r}\text { Geometrical } \\
\text { Fiducial Cut }\end{array}$ & 0.058 & 0.080 & 0.080 & 0.065 & 0.070 & 0.048 & 0.040 \\
\hline $\begin{array}{r}\text { p Acceptance } \\
\text { Corrections with } \\
\text { pol. MC }\end{array}$ & 0.086 & 0.064 & 0.080 & 0.085 & 0.080 & 0.093 & 0.075 \\
\hline Fit Method & 0.046 & 0.052 & 0.044 & 0.034 & 0.031 & 0.030 & 0.031 \\
\hline $\begin{array}{r}\text { Fixed/Float } \\
\text { Fit Parameters }\end{array}$ & 0.032 & 0.027 & 0.034 & 0.023 & 0.013 & 0.012 & 0.015 \\
\hline$\alpha$ & 0.013 & 0.013 & 0.013 & 0.013 & 0.013 & 0.013 & 0.013 \\
\hline TOTAL & 0.126 & 0.124 & 0.136 & 0.121 & 0.122 & 0.118 & 0.099 \\
\hline
\end{tabular}

Table 5.1: Summary of the systematic uncertainties. The total systematic uncertainty, assigned for each $\cos \theta_{K}^{C M} \operatorname{bin}$ is calculated as a sum of quadratures of the individual contributions. 


\section{Results and Conclusions}

\section{1 $\Lambda$ Recoil Polarization}

This chapter presents the $\Lambda$ induced polarization results for $K^{+}$electroproduction, binned in $\cos \theta_{K}^{C M}$ and $W$. Binning for this analysis is described in Section 4.2. All results are tabulated in Appendix D. The results are integrated over $\phi_{K}$ and $Q^{2}$. These results greatly extend the kinematic coverage of the only previous experimental measurement of $\Lambda$ recoil polarization for $K^{+}$electroproduction by S. McAleer [34]. The large statistics allow fine binning in $W$ without integrating over kaon angles. The $\cos \theta_{K}^{C M}$ dependence of the $\Lambda$ recoil polarization are shown in Figs. 6.1 and 6.2 along with photoproduction data [26]. One observation that can be made from these figures is that the polarization results do not change sign for any of the $W$ bins, as was observed in photoproduction data from Refs. [26]. Recall that the virtual photon in the electroproduction process possesses transverse as well as longitudinal polarization, the latter being absent in photoproduction. The cross-section data from Ref. [27] indicate a small $\sigma_{L}$. This suggests that although the longitudinal polarization of the virtual photon by itself may not play a significant role, even a small contribution in the interference terms can cause a sizable contribution in the strangeness electroproduction process for this observable.

Fig. 6.3 shows the $W$ dependence of $P_{N}$ for all $\cos \theta_{K}^{C M}$ bins. Recall that $W$ is defined as the intermediate hadronic state energy in the $s$-channel. Any structure observed in the $W$ dependence of the polarization signifies the possible contribution from a resonance with mass equal to $W$ in the production process. Similarly, the structures can be a consequence of interference from two or more broad resonance contributions that have a mass around the $W$ value where the bumps and dips appear. As one can see from these plots, the polarization varies smoothly for the backward kaon angle bins, while at medium angles, where the $s$-channel process is expected to dominate, some struc- 

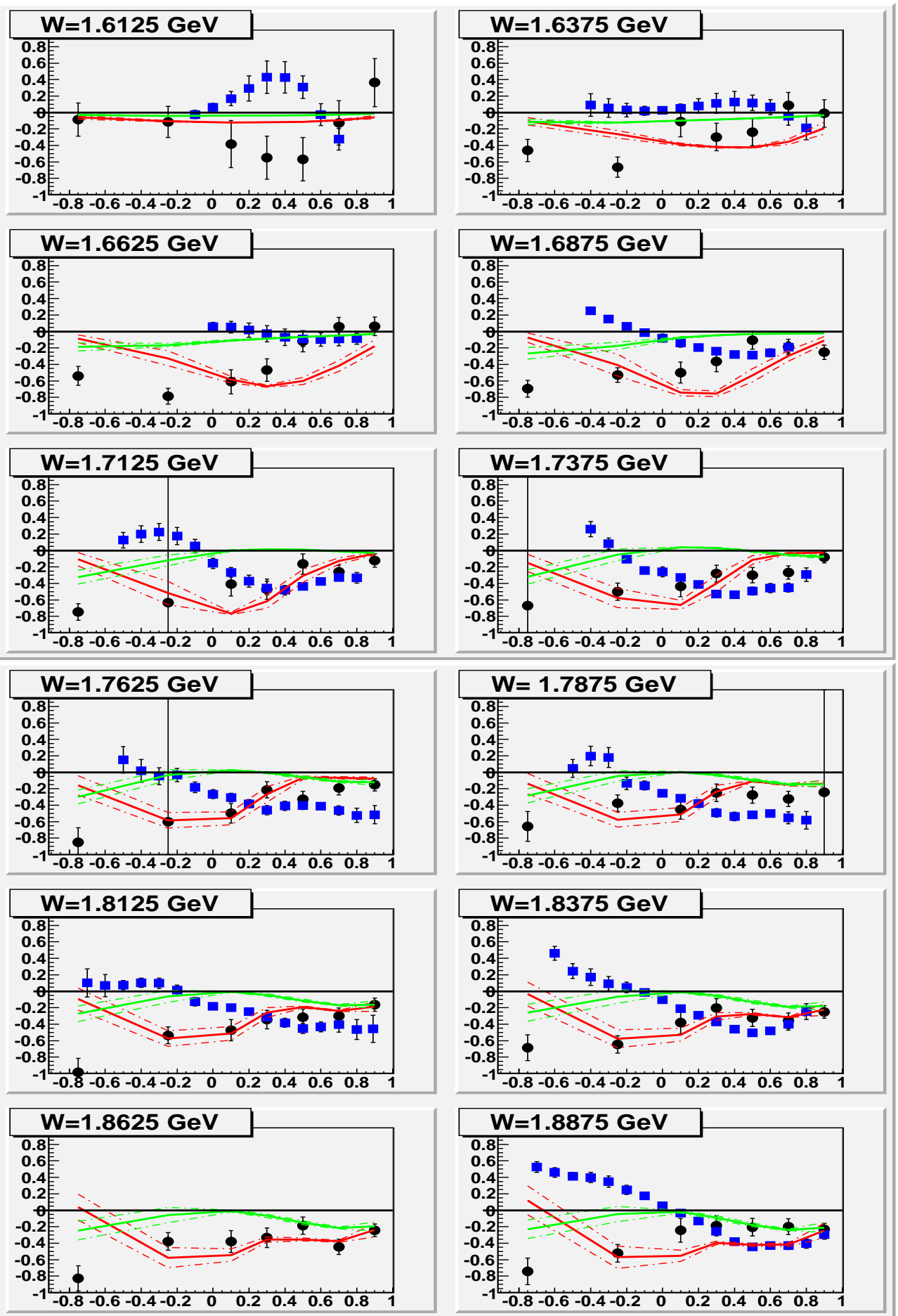

Figure 6.1: $\Lambda$ induced polarization $P_{N}$ vs. $\cos \theta_{K}^{C M}$. The black circles are the results of this analysis, the blue squares are the photoproduction results from Ref. [26]. The overlaid curves correspond to RPR [9] (green) and RPR2011 [10] (red) model predictions, respectively. The dashed curves give the upper and lower limits of the predicted polarizations using the uncertainties of the model calculations. 

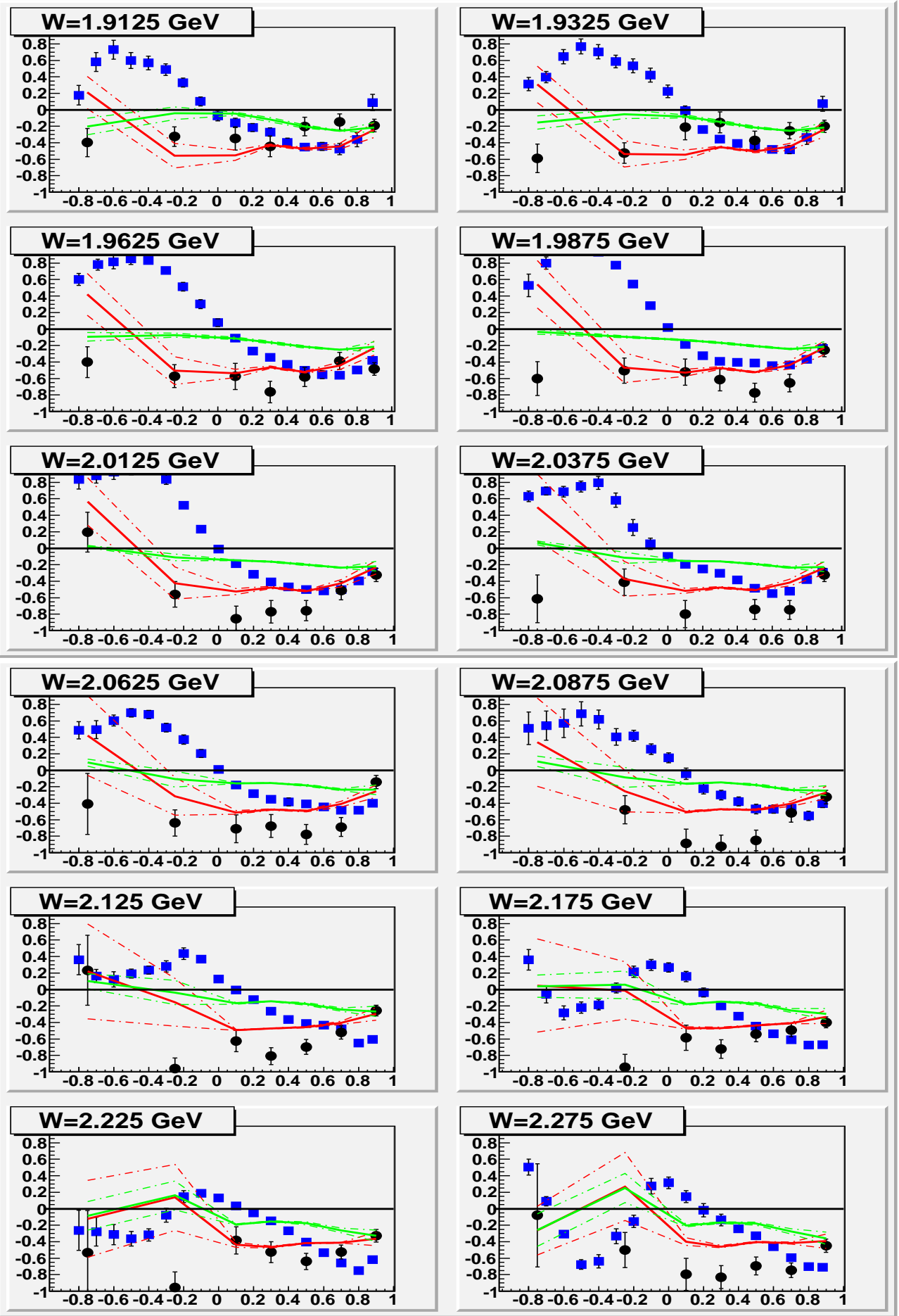

Figure 6.2: $\Lambda$ induced polarization $P_{N}$ vs. $\cos \theta_{K}^{C M}$. The black circles are the results of this analysis, the blue squares are the photoproduction results from Ref. [26]. The overlaid curves correspond to RPR [9] (green) and RPR2011 [10] (red) model predictions, respectively. The dashed curves give the upper and lower limits of the predicted polarizations using the uncertainties of the model calculations. 

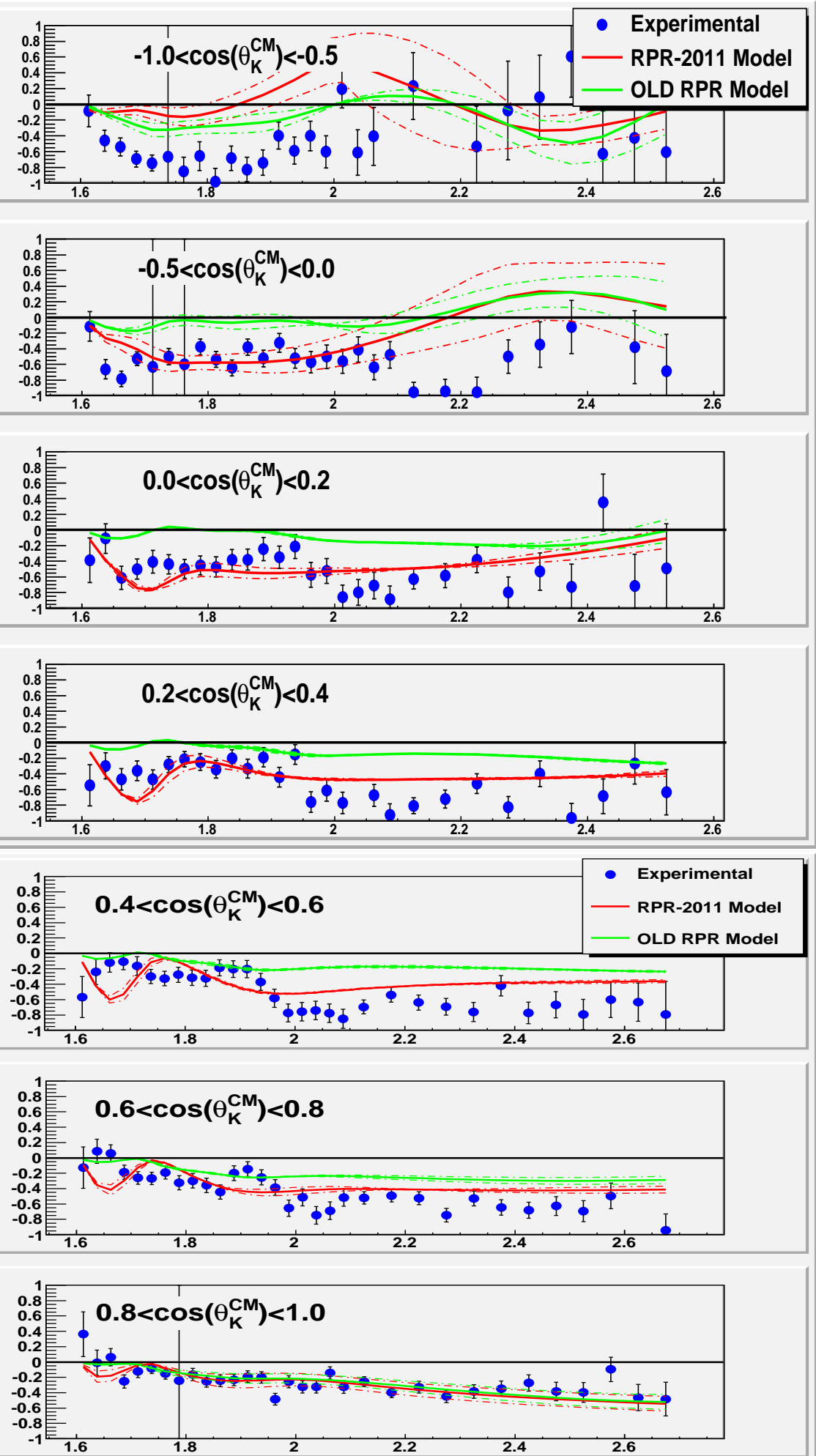

Figure 6.3: RPR [9] and RPR-2011 [10] model predictions overlaid on the induced polarization results vs. $W$. The green curve corresponds to the RPR model and the red curve to the RPR-2011 model. The dashed curves give the upper and lower limits of the predicted polarizations using the uncertainties of the model calculations. 

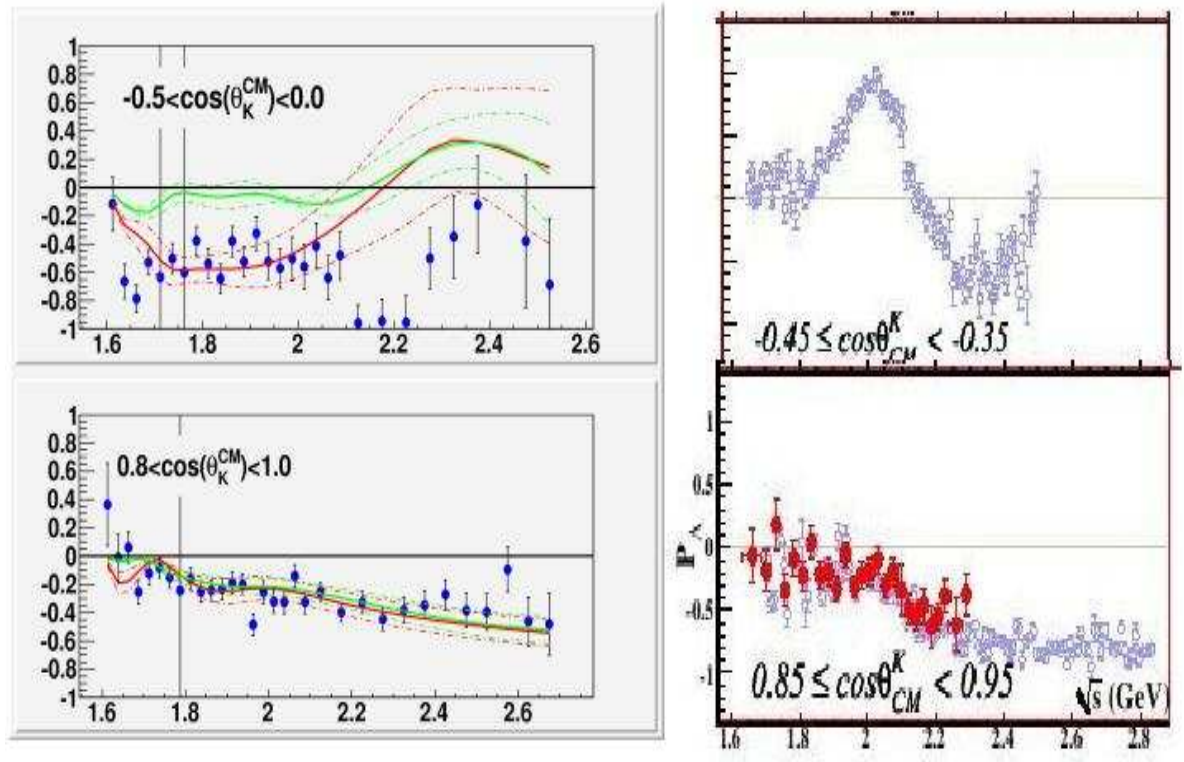

Figure 6.4: Induced polarization $P_{N}$ vs. $W$ for electro- (left) and photoproduction (right). The photoproduction results are from Ref. [26]. The sharp change of the polarization sign present in the photoproduction data at backward kaon angles is not observed in the electroproduction data. At forward kaon angles the structures vanish for both photo- and electroproduction.

ture starts to build up with a clear bump around $W=1.9 \mathrm{GeV}$ at $0.4<\cos \theta_{K}^{C M}<0.6$ and $0.6<\cos \theta_{K}^{C M}<0.8$. At very forward kaon angles, where $t$-channel processes dominate, the polarization is again flat and smaller in magnitude. Similar observations can be made from the recent photoproduction data of Ref. [26], where at backward and medium kaon angles, the polarization shows some structure, which gradually flattens out at forward kaon angles. The polarization sharply changes sign in the photoproduction data, which is not observed in the electroproduction data as can be seen from Fig. 6.4.

\subsection{Comparison to Theoretical Models}

Our polarization results are compared to two different RPR-model predictions referred to here as the old RPR model and the new RPR-2011 model. Both models treat the non-resonant background contributions as exchanges of kaonic Regge trajectories in 
the $t$-channel, with $K(494)$ and $K^{*}(892)$ as the dominant trajectories. Both have a rotating Regge phase. To take into account the $s$-channel contributions, these models include established $s$-channel nucleon resonances: S11(1650), P11(1710), P13(1720), P13(1900), as well as the missing resonance D13(1900). The old RPR model was fit to forward angle $\left(\cos \theta_{K}^{C M}>0\right)$ photoproduction data from CLAS, LEPS, and GRAAL [9].

The new RPR-2011 model was fit to the entire $\cos \theta_{K}^{C M}$ angular range of all recent $K^{+} \Lambda$ photoproduction data, including Ref. [26]. Furthermore it uses a consistent formalism for the description of spin-5/2 particles as described in Ref. [10]. As can be seen from Fig. 6.5, the old RPR model gives a reasonable description of the photoproduction crosssection data at forward kaon angles $\left(\cos \theta_{K}^{C M}>0\right)$, but fails at backward angles, while the RPR-2011 model shows remarkable improvement at backward kaon angles, giving an overall very good description of photoproduction data throughout the full kaon angular range.

The predictions of these two models, along with their uncertainties, are overlaid on our polarization results as shown in Figs. 6.1- 6.3. From Fig. 6.3 one can see that both RPR theoretical model calculations are in good agreement with the experimental data at very forward kaon angles, but the old RPR predictions fail to reproduce the data at all other kaon angle bins. The RPR-2011 reproduces the overall trend of the polarization in all but the most backward kaon angle bin $\left(-1.0<\cos \theta_{K}^{C M}<-0.5\right)$, but fails to reproduce the structure, particularly around $W=1.9 \mathrm{GeV}$.

\subsection{Comparison to Previous Experimental Results}

In this section the results are compared to the previous $\Lambda$ recoil polarization measurement results for $K^{+}$electroproduction of Ref. [34]. The data points from Ref. [34], overlaid on our results for the overlapping $W$ and $\cos \theta_{K}^{C M}$ ranges are shown in Figs. 6.66.7. The data from Ref. [34] are integrated over all $\theta_{K}^{C M}$ angles in order to improve statistics. Since the results are dominated by forward angle data because of the large cross section, the data are overlaid only on our $0.8<\cos \theta_{K}^{C M}<1.0$ bin in Fig. 6.6, 


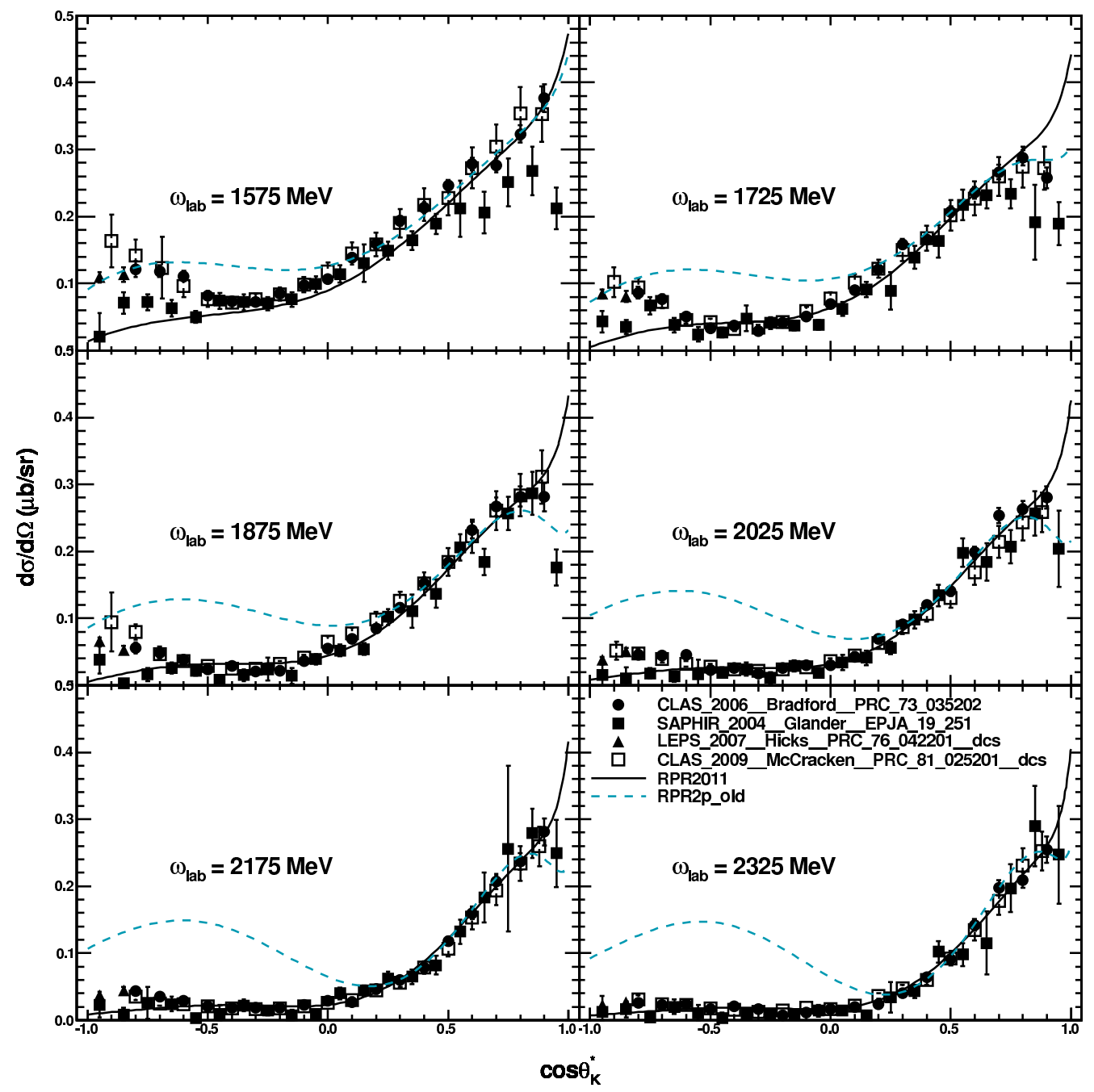

Figure 6.5: RPR and RPR-2011 model fits to all existing photoproduction data. RPR-2011 (black curve) shows remarkable improvement in the $\cos \theta_{K}^{C M}<0$ range. [10]

when plotting the $W$ dependence. The red squares are systematically lower than our data points, but this is driven by the integration of data from all $\cos \theta_{K}^{C M}$ angular bins.

Ref. [34] presents the $\cos \theta_{K}^{C M}$ dependence of the polarization for three different $W$ ranges: $1.67<W<1.76 \mathrm{GeV}, 1.716<W<1.783 \mathrm{GeV}$ and $1.873<W<2.152 \mathrm{GeV}$. The results are again integrated over $Q^{2}$ and $\phi_{K}$. In Fig. 6.7 the results from the last two $W$ bins are overlaid on our polarization results for the $W$ bins that are closer to the bin center of the covered ranges. It is surprising to see that the overall behavior and magnitude of the polarization from the old measurement is very consistent with 


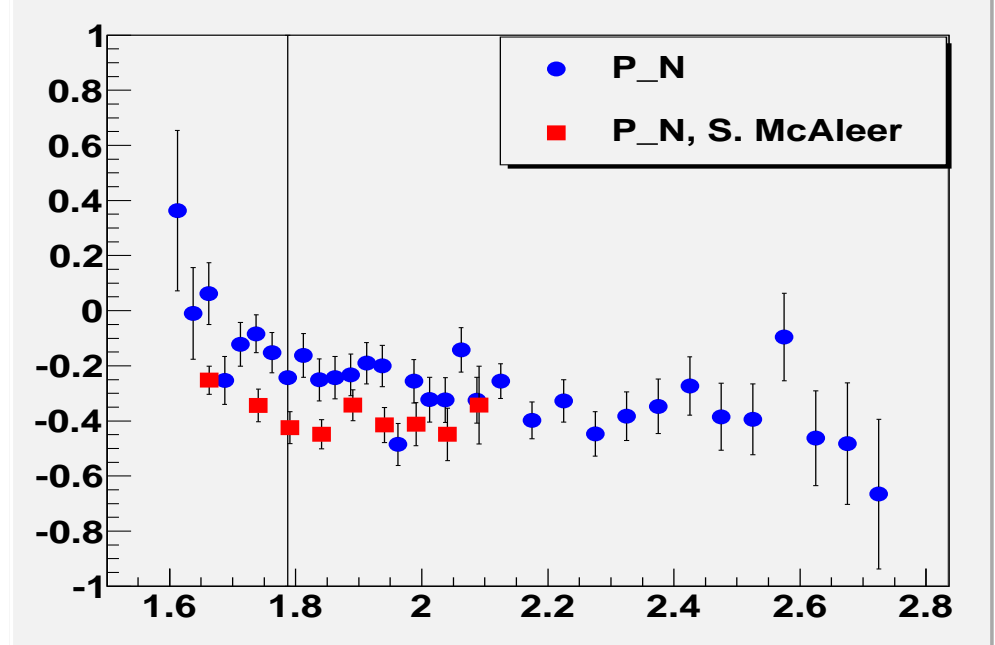

Figure 6.6: Induced polarization $P_{N}$ vs. $W$ results for $0.8<\cos \theta_{K}^{C M}<$ 1.0 kaon angles. The red squares are taken from Ref. [34]. Data from this previous measurement are integrated over all kaon angles.

our results, despite the fact that data with different beam energies and magnetic field settings were combined to achieve reasonable statistics in the old measurement.

\subsection{Conclusions}

Here we have presented $\Lambda$ recoil polarization results for $K^{+}$electroproduction for the total of $215\left(\cos \theta_{K}^{C M}, W\right)$ bins, covering a $W$ range from threshold up to $2.7 \mathrm{GeV}$ and the full kaon center of mass angular range. Our results greatly increase the kinematic range of the only existing recoil polarization electroproduction data by S. McAleer, extending it by about $600 \mathrm{MeV}$, and presenting data points at seven different $\cos \theta_{K}^{C M}$ bins.

The polarization results demonstrate structure buildup at medium kaon center of mass angles, perhaps indicating $s$-channel resonance contribution around $W=1.9 \mathrm{GeV}$. Enhancement around $W=1.9 \mathrm{GeV}$ was also observed in the $K^{+} \Lambda$ photoproduction cross section data of Refs. [24] and [26]. At the moment none of the available theoretical models is able to fully explain our results. The predictions of both RPR theoretical models are in good agreement with experimental data at very forward kaon angles, but 

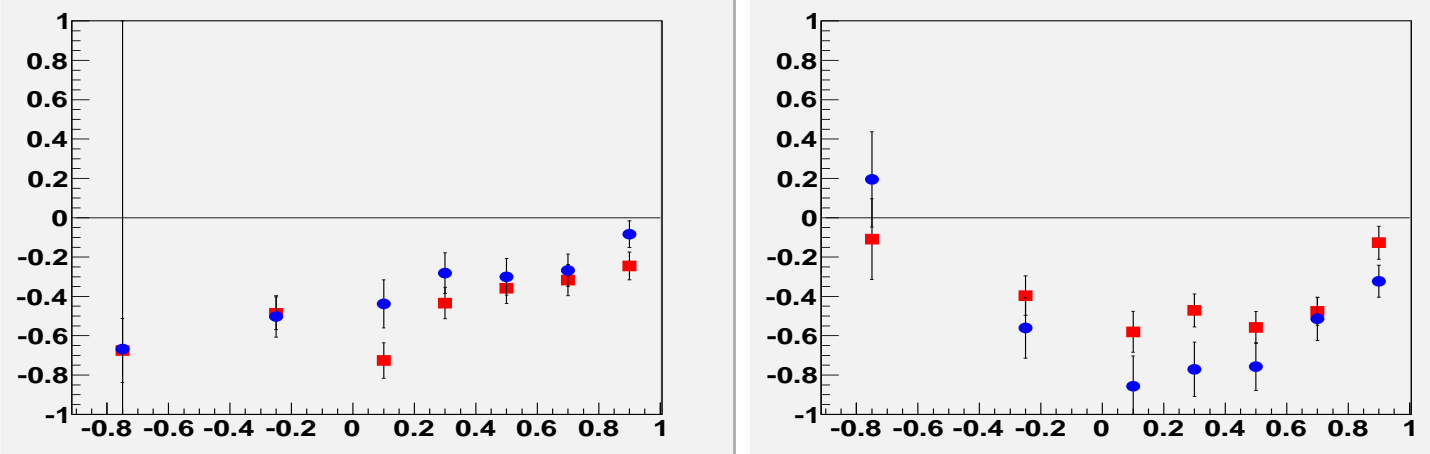

Figure 6.7: $\cos \theta_{K}^{C M}$ dependence of the induced polarization $P_{N}$. The red squares are taken from Ref. [34]. The presented data from Ref. [34] cover $1.67<W<1.76 \mathrm{GeV}$ and $1.873<W<2.152 \mathrm{GeV}$ ranges in the left and right plots, respectively. Only one of the overlapping $W$ bins is presented for each range.

the old RPR predictions fail to reproduce the data for the rest of the kaon angle range. RPR-2011 reproduces the overall the trend of the polarization in general, but fails to reproduce in detail the observed features. These results can be used to better constrain the model parameters in the future. A full partial wave analysis is necessary to interpret this enhancement and to determine the quantum numbers of the resonances contributing into the electroproduction process. 


\section{APPENDICES}




\section{A Response Functions}

\begin{tabular}{|c|c||c|c|c|c|c|c||c|c|c|}
\hline$\beta$ & $\alpha$ & $\mathrm{T}$ & $\mathrm{L}$ & ${ }^{c} \mathrm{TL}$ & ${ }^{s} \mathrm{TL}$ & ${ }^{c} \mathrm{TT}$ & ${ }^{s} \mathrm{TT}$ & ${ }^{c} \mathrm{TL}^{\prime}$ & ${ }^{s} \mathrm{TL}^{\prime}$ & $\mathrm{TT}^{\prime}$ \\
\hline \hline- & - & $\mathrm{R}_{T}^{00}$ & $\mathrm{R}_{L}^{00}$ & $\mathrm{R}_{T L}^{00}$ & 0 & $\mathrm{R}_{T T}^{00}$ & 0 & 0 & $\mathrm{R}_{T L^{\prime}}^{00}$ & 0 \\
\hline- & $\mathrm{x}$ & 0 & 0 & 0 & $\mathrm{R}_{T L}^{0 x}$ & 0 & $\mathrm{R}_{T T}^{0 x}$ & $\mathrm{R}_{T L^{\prime}}^{0 x}$ & 0 & $\mathrm{R}_{T T^{\prime}}^{0 x}$ \\
- & $\mathrm{y}$ & $\mathrm{R}_{T}^{0 y}$ & $\mathrm{R}_{L}^{0 y}$ & $\mathrm{R}_{T L}^{0 y}$ & 0 & $\ddagger$ & 0 & 0 & $\mathrm{R}_{T L^{\prime}}^{0 y}$ & 0 \\
- & $\mathrm{z}$ & 0 & 0 & 0 & $\mathrm{R}_{T L}^{0 z}$ & 0 & $\mathrm{R}_{T T}^{0 z}$ & $\mathrm{R}_{T L^{\prime}}^{0 z}$ & 0 & $\mathrm{R}_{T T^{\prime}}^{0 z}$ \\
\hline $\mathrm{x}^{\prime}$ & - & 0 & 0 & 0 & $\mathrm{R}_{T L}^{x^{\prime} 0}$ & 0 & $\mathrm{R}_{T T}^{x^{\prime} 0}$ & $\mathrm{R}_{T L^{\prime}}^{x^{\prime} 0}$ & 0 & $\mathrm{R}_{T T^{\prime}}^{x^{\prime} 0}$ \\
$\mathrm{y}^{\prime}$ & - & $\mathrm{R}_{T}^{y^{\prime} 0}$ & $\ddagger$ & $\ddagger$ & 0 & $\ddagger$ & 0 & 0 & $\ddagger$ & 0 \\
$\mathrm{z}^{\prime}$ & - & 0 & 0 & 0 & $\mathrm{R}_{T L}^{z^{\prime} 0}$ & 0 & $\mathrm{R}_{T T}^{z^{\prime} 0}$ & $\mathrm{R}_{T L^{\prime}}^{z^{\prime} 0}$ & 0 & $\mathrm{R}_{T T^{\prime}}^{z^{\prime}}$ \\
$\mathrm{x}^{\prime}$ & $\mathrm{x}$ & $\mathrm{R}_{T}^{x^{\prime} x}$ & $\mathrm{R}_{L}^{x^{\prime} x}$ & $\mathrm{R}_{T L}^{x^{\prime} x}$ & 0 & $\ddagger$ & 0 & 0 & $\mathrm{R}_{T L^{\prime}}^{x^{\prime}}$ & 0 \\
$\mathrm{x}^{\prime}$ & $\mathrm{y}$ & 0 & 0 & 0 & $\ddagger$ & 0 & $\ddagger$ & $\ddagger$ & 0 & $\ddagger$ \\
$\mathrm{x}^{\prime}$ & $\mathrm{z}$ & $\mathrm{R}_{T}^{x^{\prime} z}$ & $\mathrm{R}_{L}^{x^{\prime} z}$ & $\ddagger$ & 0 & $\ddagger$ & 0 & 0 & $\ddagger$ & 0 \\
$\mathrm{y}^{\prime}$ & $\mathrm{x}$ & 0 & 0 & 0 & $\ddagger$ & 0 & $\ddagger$ & $\ddagger$ & 0 & $\ddagger$ \\
$\mathrm{y}^{\prime}$ & $\mathrm{y}$ & $\ddagger$ & $\ddagger$ & $\ddagger$ & 0 & $\ddagger$ & 0 & 0 & $\ddagger$ & 0 \\
$\mathrm{y}^{\prime}$ & $\mathrm{z}$ & 0 & 0 & 0 & $\ddagger$ & 0 & $\ddagger$ & $\ddagger$ & 0 & $\ddagger$ \\
$\mathrm{z}^{\prime}$ & $\mathrm{x}$ & $\mathrm{R}_{T}^{z^{\prime} x}$ & $\ddagger$ & $\mathrm{R}_{T L}^{z^{\prime} x}$ & 0 & $\ddagger$ & 0 & 0 & $\mathrm{R}_{T L^{\prime} x}^{z^{\prime}}$ & 0 \\
$\mathrm{z}^{\prime}$ & $\mathrm{y}$ & 0 & 0 & 0 & $\ddagger$ & 0 & $\ddagger$ & $\ddagger$ & 0 & $\ddagger$ \\
$\mathrm{z}^{\prime}$ & $\mathrm{z}$ & $\mathrm{R}_{T}^{z^{\prime} z}$ & $\ddagger$ & $\ddagger$ & 0 & $\ddagger$ & 0 & 0 & $\ddagger$ & 0 \\
\hline
\end{tabular}

Table A.1: Response functions for pseudoscalar meson production [6]. The target (recoil) polarization is indicated by $\alpha(\beta)$. The last three columns are for when the electron is polarized. $\ddagger$ indicates a response function which does not vanish but is related to other response functions. 


\section{B $\quad \theta-p$ Nominal Cuts}

$\theta-p$ two dimensional cut are defined according to Eq. 3.3 and intended to remove the inefficient and dead areas of the DC. The parameters are defined separately for each sector. The nominal cuts used for electrons are given in Table B.1. Table B.2 summarizes the nominal cuts used for hadrons. 


\begin{tabular}{|r|r|}
\hline Sector & Applied Cut \\
\hline SECTOR 1 & NO CUT \\
\hline SECTOR 2 & $\theta_{1}^{\text {min }}=36.1 \cdot\left(1+\exp \left(-2.72 \cdot\left(p_{e}-0.014\right)\right)\right)$ \\
& $\theta_{1}^{\text {max }}=38.7 \cdot\left(1+\exp \left(-2.72 \cdot\left(p_{e}-0.004\right)\right)\right)$ \\
\hline SECTOR 3 & $\theta_{1}^{\text {min }}=27.100 \cdot\left(1+\exp \left(-1.720 \cdot\left(p_{e}-0.004\right)\right)\right)$ \\
& $\theta_{1}^{\text {max }}=30.586 \cdot\left(1+\exp \left(-1.908 \cdot\left(p_{e}+0.099\right)\right)\right)$ \\
& $\theta_{2}^{\text {max }}=14.500 \cdot\left(1+\exp \left(-1.080 \cdot\left(p_{e}-0.604\right)\right)\right)$ \\
\hline SECTOR 4 & $\theta_{1}^{\text {min }}=39.720 \cdot\left(1+\exp \left(-1.982 \cdot\left(p_{e}+0.259\right)\right)\right)$ \\
& $\theta_{1}^{\text {max }}=43.082 \cdot\left(1+\exp \left(-1.982 \cdot\left(p_{e}+0.359\right)\right)\right)$ \\
& $\theta_{2}^{\text {max }}=14.500 \cdot\left(1+\exp \left(-1.080 \cdot\left(p_{e}-0.604\right)\right)\right)$ \\
\hline SECTOR 5 & $\theta_{1}^{\text {min }}=38.32 \cdot\left(1+\exp \left(-1.782 \cdot\left(p_{e}+0.259\right)\right)\right)$ \\
& $\theta_{2}^{\text {max }}=15.30 \cdot\left(1+\exp \left(-1.152 \cdot\left(p_{e}-0.447\right)\right)\right)$ \\
\hline SECTOR 6 & $\theta_{1}^{\text {min }}=38.32 \cdot\left(1+\exp \left(-1.782 \cdot\left(p_{e}+0.259\right)\right)\right)$ \\
& $\theta_{1}^{\text {max }}=40.82 \cdot\left(1+\exp \left(-1.782 \cdot\left(p_{e}+0.339\right)\right)\right)$ \\
\hline
\end{tabular}

Table B.1: $\theta_{e}-p_{e}$ nominal cuts for electrons.

\begin{tabular}{|c|c|}
\hline Sector & Applied Cut \\
\hline SECTOR 1 & $\theta_{1}^{\text {min }}=40.935 \cdot\left(1-\exp \left(-2.846 \cdot\left(p_{h}-0.041\right)\right)\right)$ \\
& $\theta_{1}^{\text {max }}=44.735 \cdot\left(1-\exp \left(-2.846 \cdot\left(p_{h}-0.041\right)\right)\right)$ \\
\hline SECTOR 2 & $\theta_{1}^{\text {min }}=49.535 \cdot\left(1-\exp \left(-3.8600 \cdot\left(p_{h}-0.0011\right)\right)\right)$ \\
& $\theta_{1}^{\text {max }}=53.535 \cdot\left(1-\exp \left(-3.8600 \cdot\left(p_{h}-0.0011\right)\right)\right)$ \\
& $\theta_{2}^{\text {min }}=27.770 \cdot\left(1-\exp \left(-3.6996 \cdot\left(p_{h}-0.5730\right)\right)\right)$ \\
& $\theta_{2}^{\text {max }}=30.770 \cdot\left(1-\exp \left(-2.9600 \cdot\left(p_{h}-0.4130\right)\right)\right)$ \\
\hline SECTOR 3 & $\theta_{1}^{\text {min }}=20.762 \cdot\left(1-\exp \left(-1.748 \cdot\left(p_{h}-0.8250\right)\right)\right)$ \\
& $\theta_{1}^{\text {max }}=23.186 \cdot\left(1-\exp \left(-1.514 \cdot\left(p_{h}-0.5780\right)\right)\right)$ \\
& $\theta_{2}^{\text {min }}=39.850 \cdot\left(1-\exp \left(-4.400 \cdot\left(p_{h}-0.2100\right)\right)\right)$ \\
& $\theta_{2}^{\text {max }}=45.350 \cdot\left(1-\exp \left(-2.900 \cdot\left(p_{h}-0.0531\right)\right)\right)$ \\
& $\theta_{3}^{\text {min }}=48.015 \cdot\left(1-\exp \left(-4.100 \cdot\left(p_{h}-0.0170\right)\right)\right)$ \\
& $\theta_{3}^{\text {max }}=52.150 \cdot\left(1-\exp \left(-4.100 \cdot\left(p_{h}-0.0017\right)\right)\right)$ \\
\hline SECTOR 4 & $\theta_{1}^{\text {min }}=31.95 \cdot\left(1-\exp \left(-4.36 \cdot\left(p_{h}-0.4170\right)\right)\right)$ \\
& $\theta_{1}^{\text {max }}=36.95 \cdot\left(1-\exp \left(-2.36 \cdot\left(p_{h}-0.1700\right)\right)\right)$ \\
& $\theta_{2}^{\text {min }}=54.95 \cdot\left(1-\exp \left(-4.10 \cdot\left(p_{h}-0.0017\right)\right)\right)$ \\
& $\theta_{2}^{\text {max }}=57.95 \cdot\left(1-\exp \left(-4.60 \cdot\left(p_{h}-0.0017\right)\right)\right)$ \\
\hline SECTOR 5 & $\theta_{1}^{\text {min }}=30.5 \cdot\left(1-\exp \left(-4.100 \cdot\left(p_{h}-0.446\right)\right)\right)$ \\
& $\theta_{1}^{\text {max }}=33.0 \cdot\left(1-\exp \left(-3.100 \cdot\left(p_{h}-0.296\right)\right)\right)$ \\
& $\theta_{2}^{\text {min }}=33.8 \cdot\left(1-\exp \left(-3.495 \cdot\left(p_{h}-0.275\right)\right)\right)$ \\
& $\theta_{2}^{\text {max }}=36.9 \cdot\left(1-\exp \left(-3.195 \cdot\left(p_{h}-0.215\right)\right)\right)$ \\
\hline SECTOR 6 & $\theta_{1}^{\text {min }}=30.52 \cdot\left(1-\exp \left(-4.42 \cdot\left(p_{h}-0.493\right)\right)\right)$ \\
& $\theta_{1}^{\text {max }}=34.72 \cdot\left(1-\exp \left(-2.72 \cdot\left(p_{h}-0.283\right)\right)\right)$ \\
\hline
\end{tabular}

Table B.2: $\theta_{h}-p_{h}$ nominal cuts for hadrons. 


\section{Parametrization of Fit Parameters}

$$
\begin{aligned}
a_{1} & =\text { free } \\
a_{2} & =(1.1135+0.000695 W) \\
a_{3} & =0.07985-0.07503 W+0.02116 W^{2} \\
a_{4} & =0.0007008-0.0002111 W \\
a_{5} & =0.01328+0.001138 W \\
a_{6} & =-0.05711+0.06734 W-0.01337 W^{2} \\
a_{7} & =\text { free } \\
a_{8} & =\text { free } \\
a_{9} & =(1.1908+0.001442 W) \\
a_{10} & =-0.04460+0.05652 W-0.01128 W^{2} \\
a_{11} & =-0.001402+0.001425 W-0.0003256 W 2 \\
a_{12} & =0.04132-0.02062 W+0.005242 W^{2} \\
a_{13} & =0.2148-0.2348 W+0.06529 W^{2} \\
a_{14} & =\text { free } \\
a_{15} & =\text { free } \\
a_{16} & =\text { free. } \\
&
\end{aligned}
$$

(C.1) 


\section{Polarization Tables}




\begin{tabular}{|c|c|c|c|c|c|c|c|}
\hline \multicolumn{8}{|c|}{$P_{N}$ POLARIZATION RESULTS } \\
\hline$W_{W} \cos \theta_{K}^{C M}$ & $(-1.0,-0.5)$ & $(-0.5,0.0)$ & $(0.0,0.2)$ & $(0.2,0.4)$ & $(0.4,0.6)$ & $(0.6,0.8)$ & $(0.8,1.0)$ \\
\hline 1.6125 & $-0.084 \pm 0.201$ & $-0.115 \pm 0.188$ & $-0.385 \pm 0.285$ & $-0.548 \pm 0.263$ & $-0.567 \pm 0.265$ & $-0.125 \pm 0.270$ & $0.364 \pm 0.291$ \\
\hline 1.6375 & $-0.462 \pm 0.136$ & $-0.663 \pm 0.122$ & $-0.109 \pm 0.188$ & $-0.298 \pm 0.168$ & $-0.240 \pm 0.164$ & $0.086 \pm 0.158$ & $-0.010 \pm 0.167$ \\
\hline 1.6625 & $-0.539 \pm 0.115$ & $-0.785 \pm 0.097$ & $-0.611 \pm 0.146$ & $-0.470 \pm 0.137$ & $-0.118 \pm 0.128$ & $0.060 \pm 0.110$ & $0.062 \pm 0.113$ \\
\hline 1.6875 & $-0.695 \pm 0.102$ & $-0.529 \pm 0.089$ & $-0.499 \pm 0.127$ & $-0.362 \pm 0.125$ & $-0.106 \pm 0.107$ & $-0.185 \pm 0.092$ & $-0.253 \pm 0.087$ \\
\hline 1.7125 & $-0.746 \pm 0.100$ & $-0.633 \pm 3.920$ & $-0.406 \pm 0.147$ & $-0.472 \pm 0.123$ & $-0.164 \pm 0.122$ & $-0.260 \pm 0.083$ & $-0.122 \pm 0.079$ \\
\hline 1.7375 & $-0.668 \pm 3.371$ & $-0.502 \pm 0.105$ & $-0.438 \pm 0.123$ & $-0.281 \pm 0.103$ & $-0.300 \pm 0.093$ & $-0.267 \pm 0.082$ & $-0.084 \pm 0.068$ \\
\hline 1.7625 & $-0.850 \pm 0.178$ & $-0.600 \pm 5.137$ & $-0.497 \pm 0.119$ & $-0.213 \pm 0.100$ & $-0.326 \pm 0.094$ & $-0.191 \pm 0.084$ & $-0.152 \pm 0.073$ \\
\hline 1.7875 & $-0.657 \pm 0.180$ & $-0.377 \pm 0.100$ & $-0.450 \pm 0.120$ & $-0.249 \pm 0.107$ & $-0.276 \pm 0.098$ & $-0.323 \pm 0.092$ & $-0.243 \pm 2.457$ \\
\hline 1.8125 & $-0.983 \pm 0.170$ & $-0.536 \pm 0.103$ & $-0.472 \pm 0.128$ & $-0.342 \pm 0.113$ & $-0.315 \pm 0.101$ & $-0.298 \pm 0.095$ & $-0.162 \pm 0.079$ \\
\hline 1.8375 & $-0.684 \pm 0.157$ & $-0.645 \pm 0.103$ & $-0.381 \pm 0.134$ & $-0.203 \pm 0.113$ & $-0.324 \pm 0.104$ & $-0.357 \pm 0.094$ & $-0.251 \pm 0.076$ \\
\hline 1.8625 & $-0.828 \pm 0.154$ & $-0.379 \pm 0.106$ & $-0.379 \pm 0.133$ & $-0.333 \pm 0.119$ & $-0.186 \pm 0.104$ & $-0.444 \pm 0.093$ & $-0.244 \pm 0.077$ \\
\hline 1.8875 & $-0.740 \pm 0.161$ & $-0.523 \pm 0.107$ & $-0.244 \pm 0.146$ & $-0.188 \pm 0.122$ & $-0.205 \pm 0.108$ & $-0.199 \pm 0.095$ & $-0.233 \pm 0.076$ \\
\hline 1.9125 & $-0.398 \pm 0.171$ & $-0.324 \pm 0.119$ & $-0.347 \pm 0.142$ & $-0.443 \pm 0.125$ & $-0.202 \pm 0.111$ & $-0.145 \pm 0.097$ & $-0.191 \pm 0.075$ \\
\hline 1.9375 & $-0.588 \pm 0.172$ & $-0.524 \pm 0.126$ & $-0.209 \pm 0.153$ & $-0.153 \pm 0.127$ & $-0.371 \pm 0.111$ & $-0.254 \pm 0.099$ & $-0.200 \pm 0.075$ \\
\hline 1.9625 & $-0.401 \pm 0.187$ & $-0.571 \pm 0.138$ & $-0.575 \pm 0.159$ & $-0.762 \pm 0.131$ & $-0.582 \pm 0.117$ & $-0.387 \pm 0.102$ & $-0.485 \pm 0.076$ \\
\hline 1.9875 & $-0.602 \pm 0.204$ & $-0.503 \pm 0.153$ & $-0.522 \pm 0.160$ & $-0.615 \pm 0.137$ & $-0.773 \pm 0.115$ & $-0.655 \pm 0.106$ & $-0.256 \pm 0.079$ \\
\hline 2.0125 & $0.195 \pm 0.242$ & $-0.560 \pm 0.154$ & $-0.857 \pm 0.155$ & $-0.770 \pm 0.138$ & $-0.757 \pm 0.121$ & $-0.514 \pm 0.111$ & $-0.323 \pm 0.081$ \\
\hline 2.0375 & $-0.612 \pm 0.290$ & $-0.412 \pm 0.162$ & $-0.799 \pm 0.164$ & $-1.011 \pm 0.141$ & $-0.742 \pm 0.120$ & $-0.747 \pm 0.114$ & $-0.324 \pm 0.081$ \\
\hline 2.0625 & $-0.407 \pm 0.369$ & $-0.639 \pm 0.159$ & $-0.710 \pm 0.169$ & $-0.676 \pm 0.140$ & $-0.777 \pm 0.120$ & $-0.688 \pm 0.113$ & $-0.142 \pm 0.081$ \\
\hline 2.0875 & $-1.544 \pm 0.461$ & $-0.478 \pm 0.171$ & $-0.886 \pm 0.173$ & $-0.924 \pm 0.140$ & $-0.851 \pm 0.124$ & $-0.516 \pm 0.113$ & $-0.325 \pm 0.083$ \\
\hline
\end{tabular}

Table D.1: $P_{N}$ polarization results binned in $\cos \theta_{K}^{C M}$ and $W$ bins. The listed are only the statistical uncertainties. 


\begin{tabular}{|c|c|c|c|c|c|c|c|c|}
\hline & & & $P_{N}$ & B & ESULTS CONT & NUED & & \\
\hline & $\underbrace{}_{W} \cos \theta_{K}^{C M}$ & $(-1.0,-0.5)$ & $(-0.5,0.0)$ & $(0.0,0.2)$ & $(0.2,0.4)$ & $(0.4,0.6)$ & $(0.6,0.8)$ & $(0.8,1.0)$ \\
\hline & 2.125 & $0.233 \pm 0.424$ & $-0.958 \pm 0.129$ & $-0.626 \pm 0.129$ & $-0.808 \pm 0.103$ & $-0.696 \pm 0.090$ & $-0.521 \pm 0.079$ & $-0.255 \pm 0.063$ \\
\hline & 2.175 & $-1.721 \pm 0.326$ & $-0.944 \pm 0.156$ & $-0.585 \pm 0.153$ & $-0.722 \pm 0.114$ & $-0.539 \pm 0.094$ & $-0.491 \pm 0.082$ & $-0.398 \pm 0.067$ \\
\hline & 2.225 & $-0.534 \pm 0.510$ & $-0.954 \pm 0.189$ & $-0.384 \pm 0.165$ & $-0.526 \pm 0.125$ & $-0.638 \pm 0.098$ & $-0.525 \pm 0.084$ & $-0.328 \pm 0.077$ \\
\hline & 2.275 & $-0.080 \pm 0.626$ & $-0.501 \pm 0.213$ & $-0.796 \pm 0.195$ & $-0.829 \pm 0.138$ & $-0.693 \pm 0.108$ & $-0.746 \pm 0.087$ & $-0.447 \pm 0.081$ \\
\hline & 2.325 & $0.089 \pm 0.533$ & $-0.348 \pm 0.291$ & $-0.530 \pm 0.238$ & $-0.401 \pm 0.164$ & $-0.761 \pm 0.126$ & $-0.529 \pm 0.096$ & $-0.383 \pm 0.089$ \\
\hline & 2.375 & $0.610 \pm 0.522$ & $-0.120 \pm 0.339$ & $-0.729 \pm 0.295$ & $-0.963 \pm 0.185$ & $-0.420 \pm 0.133$ & $-0.644 \pm 0.101$ & $-0.347 \pm 0.099$ \\
\hline & 2.425 & $-0.629 \pm 0.544$ & $-1.017 \pm 0.426$ & $0.351 \pm 0.364$ & $-0.685 \pm 0.222$ & $-0.773 \pm 0.141$ & $-0.682 \pm 0.103$ & $-0.273 \pm 0.106$ \\
\hline & 2.475 & $-0.425 \pm 0.583$ & $-0.379 \pm 0.468$ & $-0.718 \pm 0.410$ & $-0.267 \pm 0.261$ & $-0.667 \pm 0.169$ & $-0.626 \pm 0.123$ & $-0.385 \pm 0.122$ \\
\hline & 2.525 & $-0.605 \pm 0.679$ & $-0.686 \pm 0.469$ & $-0.491 \pm 0.571$ & $-0.635 \pm 0.291$ & $-0.793 \pm 0.197$ & $-0.694 \pm 0.137$ & $-0.395 \pm 0.129$ \\
\hline & 2.575 & $-0.123 \pm 0.788$ & $-0.104 \pm 0.602$ & $-0.737 \pm 0.508$ & $-0.388 \pm 0.518$ & $-0.603 \pm 0.230$ & $-0.495 \pm 0.166$ & $-0.095 \pm 0.159$ \\
\hline & 2.625 & $-0.794 \pm 0.730$ & $-0.070 \pm 0.660$ & $0.147 \pm 0.800$ & $-0.058 \pm 0.523$ & $-0.634 \pm 0.248$ & $-1.012 \pm 0.197$ & $-0.463 \pm 0.172$ \\
\hline & 2.675 & $0.087 \pm 0.839$ & $0.328 \pm 0.810$ & $-0.169 \pm 1.036$ & $0.066 \pm 0.604$ & $-0.795 \pm 0.429$ & $-0.943 \pm 0.213$ & $-0.483 \pm 0.221$ \\
\hline
\end{tabular}

Table D.2: $P_{N}$ polarization results binned in $\cos \theta_{K}^{C M}$ and $W$ bins. The listed are only the statistical uncertainties. 
$P_{L}$ POLARIZATION RESULTS

\begin{tabular}{|r|r|r|r|r|r|r|r|}
\hline \multicolumn{7}{|c|}{$P_{L}$ POLARIZATION RESULTS } \\
\hline \multicolumn{1}{|c|}{$\cos \theta_{K}^{C M}$} & $(-1.0,-0.5)$ & $(-0.5,0.0)$ & $(0.0,0.2)$ & $(0.2,0.4)$ & $(0.4,0.6)$ & $(0.6,0.8)$ & $(0.8,1.0)$ \\
\hline 1.6125 & $0.102 \pm 0.201$ & $0.074 \pm 0.188$ & $-0.797 \pm 0.276$ & $-0.477 \pm 0.261$ & $-0.281 \pm 0.267$ & $-0.192 \pm 0.269$ & $-0.469 \pm 0.286$ \\
\hline 1.6375 & $0.215 \pm 0.137$ & $0.168 \pm 0.125$ & $-0.012 \pm 0.188$ & $-0.191 \pm 0.169$ & $-0.095 \pm 0.165$ & $-0.100 \pm 0.158$ & $0.120 \pm 0.168$ \\
\hline 1.6625 & $-0.053 \pm 0.117$ & $0.074 \pm 0.100$ & $-0.205 \pm 0.151$ & $-0.056 \pm 0.140$ & $-0.006 \pm 0.128$ & $-0.116 \pm 0.111$ & $-0.182 \pm 0.112$ \\
\hline 1.6875 & $0.192 \pm 0.105$ & $-0.042 \pm 0.091$ & $-0.013 \pm 0.129$ & $-0.215 \pm 0.127$ & $0.020 \pm 0.107$ & $-0.006 \pm 0.093$ & $-0.112 \pm 0.087$ \\
\hline 1.7125 & $0.200 \pm 0.081$ & $0.130 \pm 0.094$ & $-0.084 \pm 0.149$ & $-0.095 \pm 0.129$ & $-0.233 \pm 0.726$ & $-0.079 \pm 0.085$ & $-0.053 \pm 0.074$ \\
\hline 1.7375 & $0.272 \pm 0.168$ & $-0.065 \pm 0.105$ & $-0.242 \pm 0.124$ & $-0.202 \pm 0.103$ & $-0.198 \pm 0.096$ & $-0.113 \pm 0.083$ & $0.030 \pm 0.068$ \\
\hline 1.7625 & $0.089 \pm 0.172$ & $0.060 \pm 0.102$ & $0.052 \pm 0.121$ & $-0.122 \pm 0.117$ & $-0.038 \pm 0.098$ & $-0.114 \pm 0.084$ & $0.071 \pm 0.069$ \\
\hline 1.7875 & $-0.012 \pm 0.184$ & $-0.147 \pm 0.102$ & $-0.037 \pm 0.123$ & $0.050 \pm 0.108$ & $0.028 \pm 0.099$ & $-0.131 \pm 0.092$ & $-0.041 \pm 0.070$ \\
\hline 1.8125 & $0.264 \pm 0.173$ & $0.127 \pm 0.104$ & $-0.022 \pm 0.128$ & $-0.109 \pm 0.113$ & $-0.064 \pm 0.100$ & $0.018 \pm 0.099$ & $-0.117 \pm 0.076$ \\
\hline 1.8375 & $-0.071 \pm 0.162$ & $-0.037 \pm 0.107$ & $-0.117 \pm 0.133$ & $-0.201 \pm 0.112$ & $0.063 \pm 0.103$ & $-0.036 \pm 0.096$ & $-0.134 \pm 0.078$ \\
\hline 1.8625 & $0.076 \pm 0.159$ & $-0.106 \pm 0.107$ & $0.171 \pm 0.135$ & $0.070 \pm 0.119$ & $0.193 \pm 0.104$ & $0.083 \pm 0.096$ & $-0.009 \pm 0.076$ \\
\hline 1.8875 & $-0.143 \pm 0.164$ & $-0.054 \pm 0.108$ & $-0.087 \pm 0.139$ & $0.269 \pm 0.123$ & $0.064 \pm 0.109$ & $0.117 \pm 0.096$ & $0.105 \pm 0.077$ \\
\hline 1.9125 & $0.135 \pm 0.169$ & $0.178 \pm 0.118$ & $0.411 \pm 0.142$ & $0.069 \pm 0.127$ & $0.022 \pm 0.110$ & $-0.004 \pm 0.097$ & $-0.046 \pm 0.076$ \\
\hline 1.9375 & $0.370 \pm 0.168$ & $0.189 \pm 0.127$ & $-0.029 \pm 0.154$ & $0.006 \pm 0.126$ & $0.125 \pm 0.110$ & $0.042 \pm 0.100$ & $0.031 \pm 0.076$ \\
\hline 1.9625 & $-0.496 \pm 0.191$ & $0.112 \pm 0.143$ & $0.010 \pm 0.166$ & $0.193 \pm 0.133$ & $-0.137 \pm 0.119$ & $0.036 \pm 0.103$ & $-0.057 \pm 0.077$ \\
\hline 1.9875 & $-0.019 \pm 0.206$ & $0.073 \pm 0.153$ & $0.165 \pm 0.161$ & $-0.047 \pm 0.137$ & $-0.183 \pm 0.117$ & $0.113 \pm 0.107$ & $0.066 \pm 0.078$ \\
\hline 2.0125 & $0.358 \pm 0.231$ & $-0.164 \pm 0.154$ & $0.235 \pm 0.161$ & $-0.016 \pm 0.142$ & $0.059 \pm 0.125$ & $0.232 \pm 0.111$ & $0.277 \pm 0.080$ \\
\hline 2.0375 & $0.093 \pm 0.293$ & $0.103 \pm 0.164$ & $-0.386 \pm 0.171$ & $0.086 \pm 0.147$ & $-0.143 \pm 0.122$ & $-0.093 \pm 0.117$ & $0.178 \pm 0.083$ \\
\hline 2.0625 & $0.141 \pm 0.362$ & $-0.079 \pm 0.162$ & $-0.075 \pm 0.173$ & $0.163 \pm 0.145$ & $0.058 \pm 0.122$ & $0.108 \pm 0.114$ & $0.083 \pm 0.080$ \\
\hline 2.0875 & $0.126 \pm 0.510$ & $0.169 \pm 0.177$ & $-0.188 \pm 0.177$ & $-0.106 \pm 0.144$ & $0.043 \pm 0.127$ & $0.156 \pm 0.118$ & $0.159 \pm 0.084$ \\
\hline
\end{tabular}

Table D.3: $P_{L}$ polarization results binned in $\cos \theta_{K}^{C M}$ and $W$ bins. The listed are only the statistical uncertainties. 
$P_{L}$ POLARIZATION RESULTS CONTINUED

\begin{tabular}{|c|c|c|c|c|c|c|c|}
\hline \multicolumn{8}{|c|}{$P_{L}$ POLARIZATION RESULTS CONTINUED } \\
\hline$W_{W} \cos \theta_{K}^{C M}$ & $(-1.0,-0.5)$ & $(-0.5,0.0)$ & $(0.0,0.2)$ & $(0.2,0.4)$ & $(0.4,0.6)$ & $(0.6,0.8)$ & $(0.8,1.0)$ \\
\hline 2.125 & $-0.027 \pm 0.442$ & $0.028 \pm 0.134$ & $-0.025 \pm 0.133$ & $-0.159 \pm 0.106$ & $0.278 \pm 0.093$ & $-0.008 \pm 0.081$ & $0.103 \pm 0.064$ \\
\hline 2.175 & $0.083 \pm 0.450$ & $0.135 \pm 0.166$ & $-0.071 \pm 0.156$ & $-0.095 \pm 0.116$ & $0.045 \pm 0.095$ & $0.163 \pm 0.084$ & $0.166 \pm 0.067$ \\
\hline 2.225 & $-0.290 \pm 0.538$ & $0.087 \pm 0.191$ & $0.211 \pm 0.164$ & $-0.056 \pm 0.127$ & $-0.075 \pm 0.100$ & $-0.089 \pm 0.085$ & $0.098 \pm 0.077$ \\
\hline 2.275 & $0.048 \pm 0.654$ & $0.223 \pm 0.217$ & $-0.261 \pm 0.202$ & $0.178 \pm 0.145$ & $0.179 \pm 0.110$ & $0.059 \pm 0.090$ & $-0.067 \pm 0.080$ \\
\hline 2.325 & $-0.810 \pm 0.553$ & $0.147 \pm 0.302$ & $-1.058 \pm 0.244$ & $-0.071 \pm 0.166$ & $-0.093 \pm 0.131$ & $-0.129 \pm 0.099$ & $0.124 \pm 0.090$ \\
\hline 2.375 & $-0.738 \pm 0.608$ & $-0.330 \pm 0.333$ & $-0.551 \pm 0.287$ & $-0.115 \pm 0.194$ & $0.128 \pm 0.134$ & $0.142 \pm 0.102$ & $0.115 \pm 0.098$ \\
\hline 2.425 & $-1.467 \pm 0.503$ & $-1.035 \pm 0.526$ & $-0.612 \pm 0.332$ & $0.010 \pm 0.230$ & $0.081 \pm 0.145$ & $0.051 \pm 0.105$ & $-0.004 \pm 0.106$ \\
\hline 2.475 & $-0.493 \pm 0.612$ & $-0.569 \pm 0.434$ & $-0.574 \pm 0.453$ & $-0.303 \pm 0.262$ & $0.310 \pm 0.170$ & $0.118 \pm 0.125$ & $0.010 \pm 0.123$ \\
\hline 2.525 & $-0.301 \pm 0.756$ & $-1.299 \pm 0.535$ & $-0.222 \pm 0.579$ & $-0.064 \pm 0.298$ & $-0.023 \pm 0.196$ & $0.042 \pm 0.140$ & $0.268 \pm 0.131$ \\
\hline 2.575 & $-0.514 \pm 1.001$ & $-0.205 \pm 0.685$ & $0.058 \pm 0.586$ & $0.158 \pm 0.468$ & $-0.063 \pm 0.231$ & $0.233 \pm 0.169$ & $-0.001 \pm 0.155$ \\
\hline 2.625 & $-0.742 \pm 1.005$ & $-1.481 \pm 0.691$ & $-0.690 \pm 0.791$ & $-0.255 \pm 0.525$ & $-0.190 \pm 0.254$ & $0.615 \pm 0.205$ & $0.188 \pm 0.180$ \\
\hline 2.675 & $-0.842 \pm 1.258$ & $-1.442 \pm 1.091$ & $-0.245 \pm 1.067$ & $-0.869 \pm 0.695$ & $-0.036 \pm 0.438$ & $0.263 \pm 0.205$ & $0.494 \pm 0.231$ \\
\hline
\end{tabular}

Table D.4: $P_{L}$ polarization results binned in $\cos \theta_{K}^{C M}$ and $W$ bins. The listed are only the statistical uncertainties. 
$P_{T}$ POLARIZATION RESULTS

\begin{tabular}{|r|r|r|r|r|r|r|r|}
\hline \multicolumn{7}{|c|}{$P_{T}$ POLARIZATION RESULTS } \\
\hline \multicolumn{1}{|c|}{$\cos \theta_{K}^{C M}$} & $(-1.0,-0.5)$ & $(-0.5,0.0)$ & $(0.0,0.2)$ & $(0.2,0.4)$ & $(0.4,0.6)$ & $(0.6,0.8)$ & $(0.8,1.0)$ \\
\hline 1.6125 & $0.147 \pm 0.201$ & $0.028 \pm 0.188$ & $0.439 \pm 0.284$ & $0.267 \pm 0.265$ & $0.869 \pm 0.258$ & $0.437 \pm 0.269$ & $0.074 \pm 0.299$ \\
\hline 1.6375 & $0.316 \pm 0.137$ & $0.191 \pm 0.125$ & $0.006 \pm 0.188$ & $0.343 \pm 0.167$ & $0.189 \pm 0.164$ & $0.084 \pm 0.158$ & $0.128 \pm 0.170$ \\
\hline 1.6625 & $-0.030 \pm 0.117$ & $-0.142 \pm 0.099$ & $-0.174 \pm 0.149$ & $-0.002 \pm 0.139$ & $-0.004 \pm 0.128$ & $0.138 \pm 0.110$ & $0.201 \pm 0.114$ \\
\hline 1.6875 & $0.089 \pm 0.105$ & $0.270 \pm 0.090$ & $0.051 \pm 0.129$ & $0.300 \pm 0.125$ & $0.230 \pm 0.107$ & $0.133 \pm 0.092$ & $0.121 \pm 0.088$ \\
\hline 1.7125 & $0.132 \pm 0.135$ & $0.018 \pm 4.901$ & $0.297 \pm 0.143$ & $0.206 \pm 0.128$ & $0.098 \pm 0.094$ & $-0.121 \pm 0.052$ & $0.116 \pm 0.075$ \\
\hline 1.7375 & $0.254 \pm 0.153$ & $0.273 \pm 0.099$ & $0.075 \pm 0.122$ & $0.162 \pm 0.107$ & $0.045 \pm 0.099$ & $0.109 \pm 0.082$ & $0.140 \pm 0.069$ \\
\hline 1.7625 & $0.469 \pm 0.173$ & $0.104 \pm 0.099$ & $0.129 \pm 0.116$ & $0.200 \pm 0.115$ & $-0.092 \pm 0.100$ & $-0.128 \pm 3.341$ & $0.036 \pm 0.069$ \\
\hline 1.7875 & $0.302 \pm 0.181$ & $0.002 \pm 0.103$ & $0.212 \pm 0.122$ & $-0.187 \pm 0.109$ & $0.071 \pm 4.532$ & $-0.261 \pm 0.094$ & $-0.015 \pm 0.072$ \\
\hline 1.8125 & $-0.189 \pm 0.192$ & $0.006 \pm 0.104$ & $-0.189 \pm 0.128$ & $0.104 \pm 0.112$ & $0.099 \pm 0.103$ & $-0.082 \pm 0.099$ & $0.020 \pm 0.077$ \\
\hline 1.8375 & $-0.124 \pm 0.163$ & $0.051 \pm 0.104$ & $-0.360 \pm 0.134$ & $-0.251 \pm 0.117$ & $-0.026 \pm 0.104$ & $-0.099 \pm 0.096$ & $0.019 \pm 0.078$ \\
\hline 1.8625 & $0.149 \pm 0.159$ & $-0.027 \pm 0.108$ & $-0.152 \pm 0.136$ & $-0.110 \pm 0.122$ & $0.122 \pm 0.105$ & $-0.017 \pm 0.095$ & $-0.033 \pm 0.076$ \\
\hline 1.8875 & $-0.196 \pm 0.164$ & $-0.048 \pm 0.109$ & $-0.069 \pm 0.138$ & $0.179 \pm 0.124$ & $0.015 \pm 0.111$ & $0.173 \pm 0.095$ & $0.101 \pm 0.076$ \\
\hline 1.9125 & $0.086 \pm 0.172$ & $0.007 \pm 0.118$ & $-0.001 \pm 0.145$ & $-0.097 \pm 0.128$ & $-0.041 \pm 0.113$ & $0.089 \pm 0.097$ & $0.033 \pm 0.075$ \\
\hline 1.9375 & $0.053 \pm 0.171$ & $0.137 \pm 0.128$ & $-0.322 \pm 0.154$ & $0.298 \pm 0.128$ & $0.024 \pm 0.112$ & $-0.005 \pm 0.100$ & $0.128 \pm 0.076$ \\
\hline 1.9625 & $0.360 \pm 0.191$ & $0.018 \pm 0.139$ & $0.159 \pm 0.164$ & $0.127 \pm 0.136$ & $0.016 \pm 0.120$ & $-0.130 \pm 0.103$ & $0.131 \pm 0.077$ \\
\hline 1.9875 & $0.095 \pm 0.209$ & $0.003 \pm 0.155$ & $-0.269 \pm 0.165$ & $0.150 \pm 0.138$ & $0.155 \pm 0.117$ & $-0.019 \pm 0.107$ & $0.057 \pm 0.079$ \\
\hline 2.0125 & $0.354 \pm 0.232$ & $0.201 \pm 0.155$ & $-0.060 \pm 0.161$ & $0.133 \pm 0.143$ & $0.194 \pm 0.123$ & $0.044 \pm 0.114$ & $0.009 \pm 0.081$ \\
\hline 2.0375 & $-0.042 \pm 0.290$ & $0.113 \pm 0.163$ & $-0.049 \pm 0.170$ & $0.169 \pm 0.146$ & $0.077 \pm 0.124$ & $-0.057 \pm 0.116$ & $0.170 \pm 0.083$ \\
\hline 2.0625 & $0.174 \pm 0.353$ & $0.090 \pm 0.160$ & $0.159 \pm 0.173$ & $0.166 \pm 0.143$ & $-0.348 \pm 0.123$ & $0.081 \pm 0.116$ & $0.051 \pm 0.082$ \\
\hline 2.0875 & $0.437 \pm 0.508$ & $0.129 \pm 0.176$ & $0.103 \pm 0.178$ & $0.218 \pm 0.145$ & $0.198 \pm 0.128$ & $-0.086 \pm 0.118$ & $-0.012 \pm 0.083$ \\
\hline
\end{tabular}

Table D.5: $P_{T}$ polarization results binned in $\cos \theta_{K}^{C M}$ and $W$ bins. The listed are only the statistical uncertainties. 
$P_{T}$ POLARIZATION RESULTS CONTINUED

\begin{tabular}{|c|c|c|c|c|c|c|c|}
\hline \multicolumn{8}{|c|}{$P_{T}$ POLARIZATION RESULTS CONTINUED } \\
\hline$W_{W} \cos \theta_{K}^{C M}$ & $(-1.0,-0.5)$ & $(-0.5,0.0)$ & $(0.0,0.2)$ & $(0.2,0.4)$ & $(0.4,0.6)$ & $(0.6,0.8)$ & $(0.8,1.0)$ \\
\hline 2.125 & $0.664 \pm 0.426$ & $0.041 \pm 0.133$ & $0.043 \pm 0.133$ & $0.053 \pm 0.107$ & $0.237 \pm 0.092$ & $0.138 \pm 0.080$ & $-0.019 \pm 0.063$ \\
\hline 2.225 & $-0.268 \pm 0.505$ & $0.126 \pm 0.188$ & $0.213 \pm 0.162$ & $0.056 \pm 0.126$ & $-0.021 \pm 0.101$ & $0.127 \pm 0.086$ & $0.010 \pm 0.077$ \\
\hline 2.275 & $0.105 \pm 0.600$ & $-0.199 \pm 0.221$ & $0.234 \pm 0.205$ & $0.165 \pm 0.145$ & $0.013 \pm 0.110$ & $-0.068 \pm 0.089$ & $0.043 \pm 0.082$ \\
\hline 2.325 & $-0.176 \pm 0.676$ & $-0.243 \pm 0.295$ & $0.214 \pm 0.238$ & $0.395 \pm 0.164$ & $0.116 \pm 0.129$ & $-0.010 \pm 0.097$ & $-0.087 \pm 0.091$ \\
\hline 2.475 & $-0.247 \pm 0.550$ & $-0.380 \pm 0.437$ & $-0.870 \pm 0.429$ & $-0.031 \pm 0.262$ & $0.234 \pm 0.172$ & $-0.019 \pm 0.124$ & $-0.071 \pm 0.124$ \\
\hline 2.525 & $-0.403 \pm 0.690$ & $-0.163 \pm 0.492$ & $-0.510 \pm 0.546$ & $0.072 \pm 0.307$ & $0.192 \pm 0.190$ & $-0.024 \pm 0.140$ & $0.078 \pm 0.132$ \\
\hline 2.575 & $0.550 \pm 0.740$ & $0.052 \pm 0.584$ & $0.267 \pm 0.553$ & $-0.071 \pm 0.495$ & $0.325 \pm 0.227$ & $0.131 \pm 0.161$ & $0.107 \pm 0.160$ \\
\hline 2.625 & $0.888 \pm 0.745$ & $0.435 \pm 0.737$ & $-0.593 \pm 0.685$ & $1.404 \pm 0.540$ & $-0.357 \pm 0.255$ & $0.211 \pm 0.207$ & $-0.401 \pm 0.182$ \\
\hline 2.675 & $-0.212 \pm 0.894$ & $0.001 \pm 0.914$ & $-0.510 \pm 1.042$ & $-0.265 \pm 0.626$ & $-0.674 \pm 0.410$ & $-0.226 \pm 0.209$ & $0.260 \pm 0.226$ \\
\hline
\end{tabular}

Table D.6: $P_{T}$ polarization results binned in $\cos \theta_{K}^{C M}$ and $W$ bins. The listed are only the statistical uncertainties. 


\section{LIST OF REFERENCES}

[1] S. Sakata, Prog. Theor. Phys. Vol. 16 No. 6 (1956) pp. 686-688 2

[2] K. Mukhin. Experimental Nuclear Physics, Volume 2 1983. xii, 2, 3, 5

[3] D. Perkins. Introduction to High Energy Physics, 1972. 2, 16, 17

[4] M. Gell-Mann, The Physical Review, Vol 125, pp. 1067-1084. Lancaster, PA and New York, NY: American Institute of Physics, 19623

[5] S. Capstick and W. Roberts, Phys. Rev. D 47, 1994 (1993) 5

[6] G. Knöchlein, D. Drechsel, L. Tiator, Z. Phys. A352, 327 (1995). 7, 8, 11, 115

[7] S. Eidelman et al. (PARTICLE DATA GROUP), Review of Particle Physics, Physics Letters B 592, 1 (2004) xii, 4, 6, 7, 16, 17, 18, 19, 23, 85

[8] D. S. Carman, K. Joo, L. Kramer, B. Raue. JLab proposal E-99-006. 11

[9] T. Corthals et al., Phys. Lett. B 656 (2007) 23, 106, 107, 108, 110

[10] T. Vrancx, arXiv:1105.2688 106, 107, 108, 110, 111

[11] S. Capstick and W. Roberts, Phys. Rev. D 58, 074011 (1998). 21

[12] H. Haberzettl et al., Phys. Rev. C 58, R40 (1998); 20

[13] T. Mart and C. Bennhold, Phys. Rev. C 61, 012201 (2000). 20, 21

[14] W. Chiang et al., Phys. Rev. C 69, 065208 (2004). 21, 22

[15] W.-T. Chiang, B. Saghai, F. Tabakin, T.-S.H. Lee, Phys. Lett. B 517, 101 (2001). 21

[16] B. Saghai, AIP Conference Proceedings 594, 421 (2001). 21

[17] V. Shklyar, H. Lenske, and U. Mosel, Phys. Rev. C 72, 015210 (2005). 21, 22

[18] B. Julia-Diaz et al., Nucl. Phys. A 755, 463 (2005); B. Julia-Diaz et al., Phys. Rev. C 73, 055204 (2006). 22

[19] A.V. Sarantsev et al., Eur. Phys. J. A 25, 441 (2005). 22

[20] M. Q. Tran et al., Phys. Lett. B 445, 20 (1998). 20, 24

[21] R. G. T. Zegers et al., Phys. Rev. Lett. 91, 092001 (2003); M. Sumihama et al., Phys. Rev. C73, 035214 (2006). 24

[22] J. W. C. McNabb et al., Phys. Rev. C69, 042201(R) (2004). 20, 24

[23] A. Lleres et al., Eur. Phys. J. A31, 79 (2007); A. D’Angelo et al., ibid. 31, 441 (2007). 24 
[24] R. Bradford et al., Phys. Rev. C73, 035202 (2006). 20, 24, 112

[25] R. Bradford et al., Phys. Rev. C75, 035205 (2007). 24

[26] M.E. McCracken et al., Phys. Rev. C81, 025201 (2010). 24, 105, 106, 107, 109, 110,112

[27] P. Ambrozewicz et al., Phys. Rev. C75, 045203 (2007). 20, 23, 24, 105

[28] M. Coman et al. Phys. Rev. C81 052201, (2010). 24

[29] R. Nasseripour et al., Phys. Rev. C77, 065208 (2008). 24

[30] R. M. Mohring et al., Phys. Rev. C67, 055205 (2003). 24

[31] G. Niculescu et al., Phys. Rev. Lett. 81, 1805 (1998); 24

[32] D.S. Carman, et al., CLAS Collaboration, Phys. Rev. Lett. 90 (2003) 131804. 20, 23,24

[33] D.S. Carman, et al., CLAS Collaboration, Phys. Rev. C79, 065205 (2009). 20, 24

[34] S. McAleer, PhD dissertation, Florida State University, 2002. 25, 105, 110, 111, 112,113

[35] B. A. Mecking et al., Nucl. Instrum. Meth. A503, 513 (2003). 27, 28

[36] H. Egiyan, Ph.D. Thesis, College of William and Mary, 2001. ix, 26

[37] E. S. Smith et al., Nucl. Instr. and Meth. A432 (1999), p. 265. 36

[38] M. Amarian et al., Nucl. Instr. and Meth. A460 (2001), p. 239. 35

[39] M. D. Mestayer et al., Nucl. Instr. and Meth. A449 (2000). 28, 31

[40] G. Adams et al., Nucl. Instr. and Meth. A465 (2001). 33

[41] E. S. Smith et al., NIM A 432 (1999) 265 298. 36

[42] J. J. Manak, E. S. Smith, S. McAleer, S. Barlow, e1, g1, g6 Data Processing Procedures, CLAS-NOTE 1999-016, JLAB. 60

[43] K. Egiyan, Determination of Electron Energy cut due to the CLAS EC Threshold, CLAS-NOTE 1999-007, (1999). 45

[44] V. Kubarovsky, e1-6 z-vertex correction routine VERTEX_E16. 45

[45] S. Stepanyan, subroutine ec_fid. 46

[46] D. S. Carman, Momentum Corrections for E1 - 6, internal analysis note.

[47] M. Mirazita, Momentum Corrections for E1F, internal analysis note. 61 61 
MARIANNA Y. GABRIELYAN

$\begin{array}{ll}2010-2012 & \begin{array}{l}\text { Graduate Assistant } \\ \text { Florida International University } \\ \text { Miami, Florida }\end{array} \\ & \text { Graduate Student Research Competition. 1st place. } \\ 2010 & \text { FIU DYF Fellowship Award } \\ 2009 & \text { Graduate Assistant } \\ & \text { Florida International University } \\ & \text { Miami, Florida } \\ & \text { MS in Physics, } \\ & \text { University of Kentucky } \\ & \text { Lexington, Kentucky } \\ & \text { Graduate Assistant } \\ & \text { University of Kentucky } \\ & \text { Lexington, Kentucky } \\ & \text { Academic Excellency Award, Yerevan State University. } \\ & \text { B.S., Physics } \\ 1999 & \text { Yerevan State University } \\ & \text { Yerevan, Armenia }\end{array}$

\section{Publications and Presentations}

M.Y. Gabrielyan, B. Raue, D.S. Carman, K. Park and CLAS Collaboration. Measurement of the Induced $\Lambda(1116)$ polarization in $K^{+}$electroproduction at CLAS. The 8th International Workshop on the Physics of Excited Nucleons, Newport News, VA, 2011. GRC Photonuclear reactions (poster). Tilton, NH, August 2010.

M.Y. Gabrielyan, B. Raue, D.S. Carman, K. Park and CLAS Collaboration. Measurement of the Induced $\Lambda(1116)$ polarization in $K^{+}$electroproduction at CLAS. Submitted 
to AIP Conference Proceedings. Proceedings of NSTAR2011 - The 8th International Workshop on the Physics of Excited Nucleons, Newport News, VA, 2011.

H. Lu, R. Schumacher, B. Raue, M. Gabrielyan, Electroproduction of $\Lambda(1405)$, Proceedings of NSTAR2011 - The 8th International Workshop on the Physics of Excited Nucleons, Newport News, VA, 2011.

M.Y. Gabrielyan, B. Raue, S. Dhamija, D.S. Carman and CLAS Collaboration. Measurement of the Induced $\Lambda(1116)$ polarization in $K^{+}$electro-production with CLAS. HADRON 2009 - The XIII International Conference on Hadron Spectroscopy. Tallahassee, Florida, 2009. APS 3rd Joint Meeting of the APS Division of Nuclear Physics and the Physical Society of Japan, Waikoloa, HI, October 2009

M.Y. Gabrielyan, B. Raue, S. Dhamija, D.S. Carman and CLAS Collaboration. Measurement of the Induced $\Lambda(1116)$ polarization in $K^{+}$electro-production with CLAS. HADRON 2009 - The XIII International Conference on Hadron Spectroscopy. AIP Conference Proceedings, Volume 1257, pp. 656-660 (2010).

S. Stepanyan, B. Mecking, S. Boyarinov, H. Egiyan, L. Guo, D. Dale, M. Gabrielyan, L. Gan, A. Gasparyan, A. Teymurazyan, I. Nakagawa, A. Glamazdin, M. Wood. Energy calibration of the JLab bremsstrahlung tagging system, NIM A 572, 654 (2007).

M. Gabrielyan, D. Dale Investigation of Limitations of the Photon Tagging Technique at High Energies. Bulletin of the APS April Meeting, Dallas, TX, 2006.

M.E. McCracken et al., Phys. Rev. C81, 025201 (2010). Differential cross section and recoil polarization measurements for the $\gamma p \rightarrow K^{+} \Lambda$ reaction using CLAS at Jefferson Lab.

D. S. Carman et al. (CLAS Collaboration) Beam-recoil polarization transfer in the nucleon resonance region in the exclusive $e p \rightarrow e^{\prime} K^{+} \Lambda$ and $e p \rightarrow e^{\prime} K^{+} \Sigma^{0}$ reactions at the CLAS spectrometer. Phys. Rev. C79, 065205 (2009) 